\title{
Synthesis of Cyclic Amidines by Iridium-Catalyzed Deoxygenative Reduction of Lactams and Tandem Reaction with Sulfonyl Azides
}

\author{
Youliang He and Xiaoming Wang*
}

\section{Table of content}

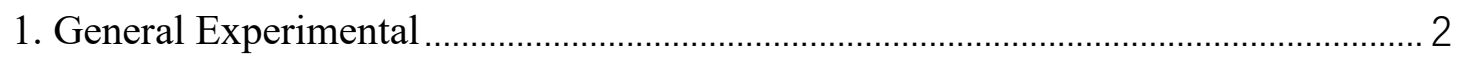

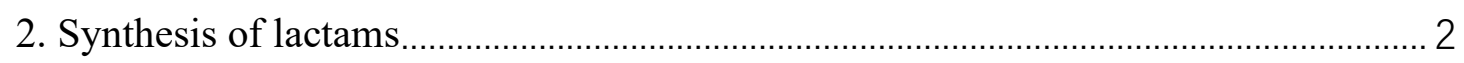

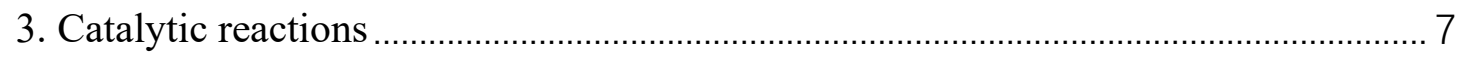

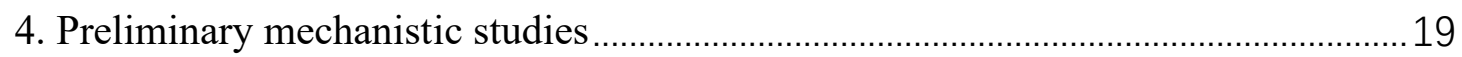

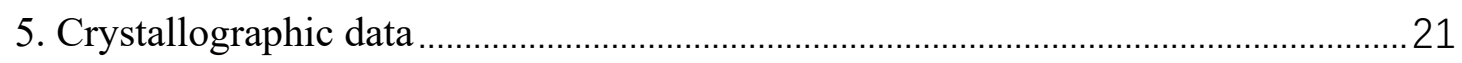

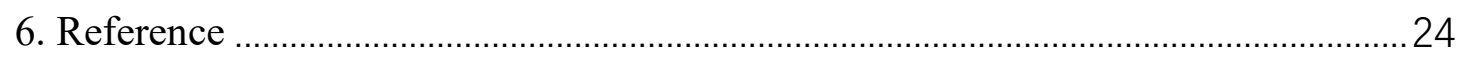

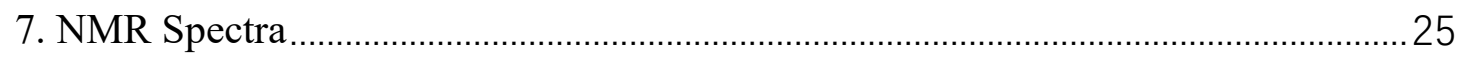




\section{General Experimental}

Unless otherwise noted, all reactions and manipulations involving air- or moisture sensitive compounds were performed using standard Schlenk techniques or in a glovebox. All solvents were purified and dried using standard procedures. ${ }^{1} \mathrm{H},{ }^{13} \mathrm{C}$ and ${ }^{19} \mathrm{~F}$ NMR spectra were recorded on Varian $400 \mathrm{MHz}$, Agilent $400 \mathrm{MHz}$ or $600 \mathrm{MHz}$ spectrometers. Chemical shifts ( $\delta$ values) were reported in ppm relative to internal TMS ( ${ }^{1} \mathrm{H}$ NMR) or $\mathrm{CDCl}_{3}\left({ }^{13} \mathrm{C}\right.$ NMR), respectively. The following abbreviations (or combinations thereof) were used to explain the multiplicities: $\mathrm{s}=$ singlet, $\mathrm{d}=$ doublet, $\mathrm{t}$ $=$ triplet, $\mathrm{q}=$ quartet, $\mathrm{m}=$ multiplet, $\mathrm{br}=$ broad. IR spectra were obtained on Bruker Tensor 27 instruments with Bruker Platinum ATR accessory. HRMS (ESI) were determined on JMS-T100LP AccuTOF LC-plus 4G. Sulfone azides were synthesized according to the literature. ${ }^{[1]}$

\section{Synthesis of lactams}

Known lactams were synthesized according to the literature. ${ }^{[2][3]}$

New compounds:
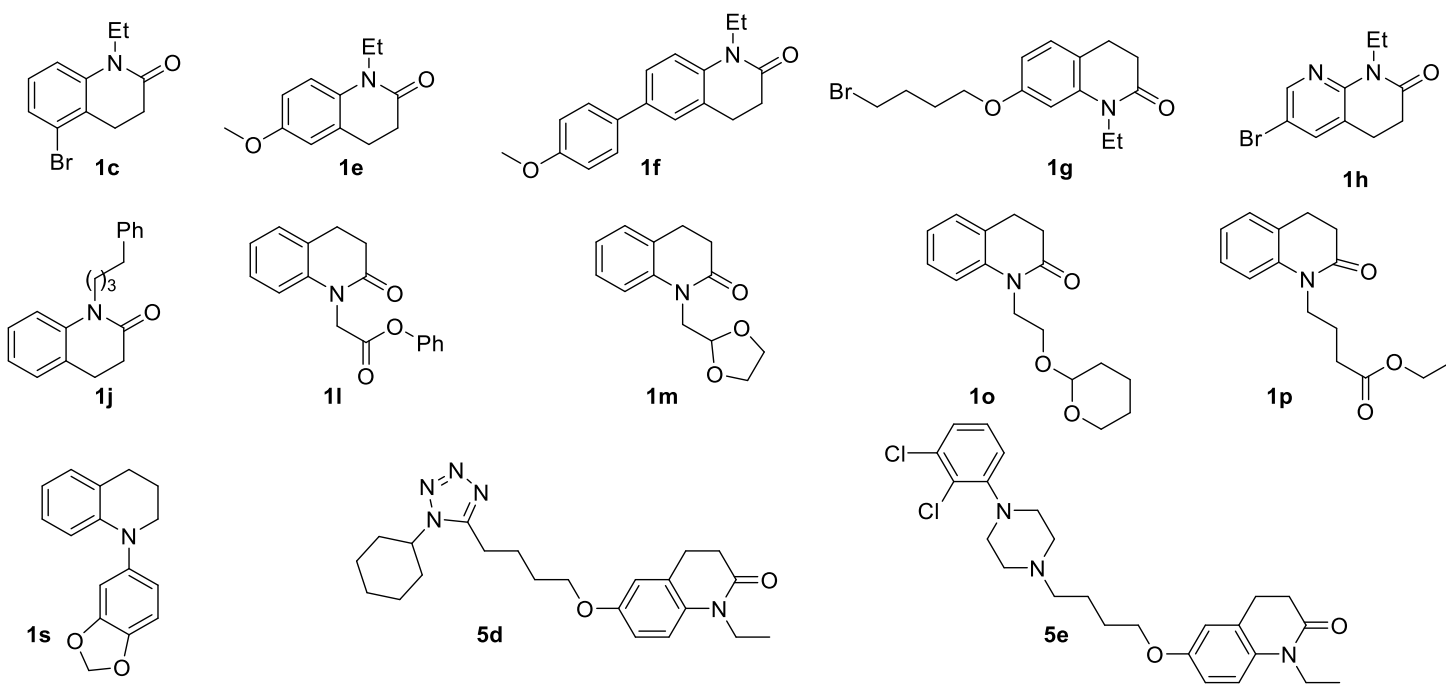

\section{General Procedure 1 (GP 1)}<smiles>O=C1CCc2ccccc2N1</smiles><smiles>[R1][R10]([H])(C)NC(C)=N</smiles><smiles>[R11]N1C(=O)CCc2ccccc21</smiles>

Under argon atmosphere, a solution of amide $(5 \mathrm{mmol})$ in dry THF $(10 \mathrm{~mL})$ was added to a suspension of sodium hydride ( $60 \%$ dispersion in mineral oil, $300 \mathrm{mg}, 6 \mathrm{mmol})$ at $0{ }^{\circ} \mathrm{C}$. The resulting reaction mixture was stirred at the same temperature for $30 \mathrm{mins}$ and then $\mathbf{R}^{\mathbf{1}}$-I ( $6 \mathrm{mmol}$ ) was added dropwise. The reaction mixture was allowed to warm to room temperature and stirred for further $12 \mathrm{~h}$. The reaction was quenched by addition 
of saturated aqueous ammonium chloride $(20 \mathrm{~mL})$. The mixture was extracted with ethyl acetate $(10 \mathrm{~mL})$ for 3 times. The combined organic phase was washed with brine, dried over anhydrous sodium sulfate and concentrated under vacuum. The residue was purified by flash chromatography on silica gel using a mixture of ethyl acetate/petroleum ether as eluent to yield lactams.

\section{General Procedure 2 (GP 2)}

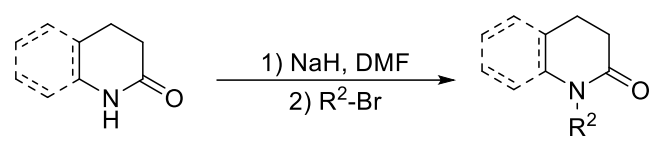

Under argon atmosphere, a solution of amide $(5 \mathrm{mmol})$ in dry THF $(10 \mathrm{~mL})$ was added to a suspension of sodium hydride (60\% dispersion in mineral oil, $240 \mathrm{~g}, 6 \mathrm{mmol}$ ) at $0{ }^{\circ} \mathrm{C}$. The resulting reaction mixture was stirred at the same temperature for 30 mins and then $\mathbf{R}^{2}$ - $\mathbf{B r}$ ( $6 \mathrm{mmol}$ ) was added dropwise. The reaction mixture was allowed to warm to $80{ }^{\circ} \mathrm{C}$ in an oil bath and stirred for further $12 \mathrm{~h}$. The reaction was quenched by addition of saturated aqueous ammonium chloride $(20 \mathrm{~mL})$. The mixture was extracted with ethyl acetate $(10 \mathrm{~mL})$ for 3 times. The combined organic phase was washed with brine, dried over anhydrous sodium sulfate and concentrated under vacuum. The residue was purified by flash chromatography on silica gel using a mixture of ethyl acetate/petroleum ether as eluent to yield lactams.

\section{5-bromo-1-ethyl-3,4-dihydroquinolin-2(1H)-one (1c)}

GP 1. 3 mmol-scale, purified by flash column chromatography (eluent: petroleum ether/ethyl acetate $=4 / 1-1 / 1$ ), white solid, $532 \mathrm{mg}, 70 \%$ yield. $\mathbf{M p} 76-77{ }^{\circ} \mathrm{C}$. ${ }^{1} \mathbf{H}$ NMR

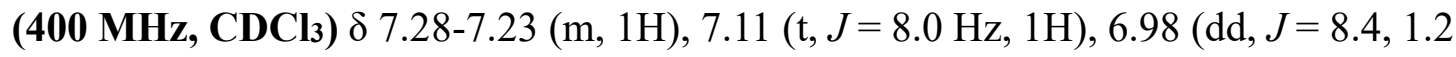
$\mathrm{Hz}, 1 \mathrm{H}), 3.98(\mathrm{q}, J=7.2 \mathrm{~Hz}, 2 \mathrm{H}), 3.05-3.01(\mathrm{~m}, 2 \mathrm{H}), 2.65-2.61(\mathrm{~m}, 2 \mathrm{H}), 1.25(\mathrm{t}, J=$ $7.2 \mathrm{~Hz}, 3 \mathrm{H}) \mathrm{ppm} ;{ }^{13} \mathbf{C}$ NMR (101 MHz, $\left.\mathbf{C D C l}_{3}\right) \delta 169.4,140.9,128.2,126.7,126.3$, 123.7, 113.9, 37.7, 31.1, 25.3, 12.6 ppm; IR (neat) v 3070, 2982, 1660, 1571, 1450, 1378, 768, $720 \mathrm{~cm}^{-1}$. HRMS (ESI) m/z: $[\mathrm{M}+\mathrm{H}]^{+}$calcd for $\mathrm{C}_{11} \mathrm{H}_{13} \mathrm{NOBr} 254.0175$; found: 254.0180 .

\section{1-ethyl-6-methoxy-3,4-dihydroquinolin-2(1H)-one (1e)}

GP 1. 3 mmol-scale, purified by flash column chromatography (eluent: petroleum ether/ethyl acetate =4/1-1/1), pale yellow oil, $521 \mathrm{mg}, 85 \%$ yield. ${ }^{1} \mathbf{H}$ NMR (400 MHz, CDCl 3$) \delta 6.94(\mathrm{~d}, J=8.8 \mathrm{~Hz}, 1 \mathrm{H}), 6.77(\mathrm{dd}, J=8.8,2.8 \mathrm{~Hz}, 1 \mathrm{H}), 6.73(\mathrm{~d}, J=3.2 \mathrm{~Hz}$, $1 \mathrm{H}), 3.96(\mathrm{q}, J=7.2 \mathrm{~Hz}, 2 \mathrm{H}), 3.79(\mathrm{~s}, 3 \mathrm{H}), 2.87-2.84(\mathrm{~m}, 2 \mathrm{H}), 2.63-2.60(\mathrm{~m}, 2 \mathrm{H}), 1.24$ $(\mathrm{t}, J=7.2 \mathrm{~Hz}, 3 \mathrm{H}) \mathrm{ppm} ;{ }^{13} \mathbf{C}$ NMR (101 MHz, $\left.\mathbf{C D C l}_{3}\right) \delta 169.4,155.1,133.0,128.1$, 115.5, 114.0, 111.9, 55.5, 37.4, 31.9, 25.8, 12.8 ppm; IR (neat) $\vee 2971,2837,1645$, 1502, 1433, 1234,1036,805 cm $\mathrm{cm}^{-1}$ HRMS (ESI) m/z: $[\mathrm{M}+\mathrm{H}]^{+}$calcd for $\mathrm{C}_{12} \mathrm{H}_{16} \mathrm{NO}_{2}$ 206.1176; found: 206.1176 .

\section{1-ethyl-6-(4-methoxyphenyl)-3,4-dihydroquinolin-2(1H)-one (1f)}



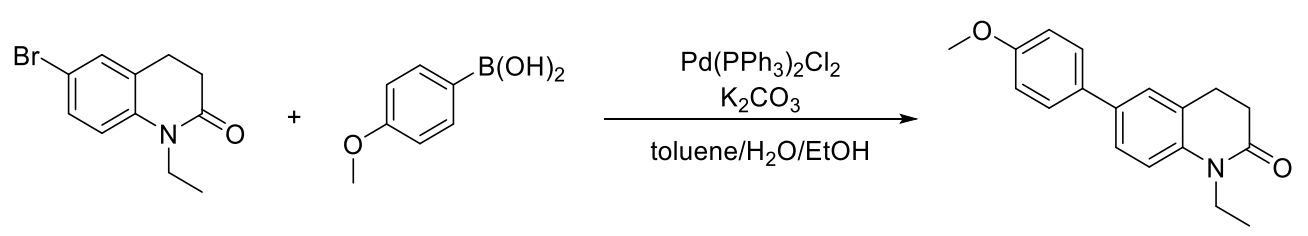

To a $50 \mathrm{~mL}$ round bottomed flask containing a stir bar, $\mathrm{Ar}-\mathrm{B}(\mathrm{OH})_{2}(4 \mathrm{mmol}), \mathbf{1 b}(2$ $\mathrm{mmol}), \mathrm{K}_{2} \mathrm{CO}_{3}(552 \mathrm{mg}, 4 \mathrm{mmol})$, and $\mathrm{Pd}\left(\mathrm{PPh}_{3}\right)_{2} \mathrm{Cl}_{2}(28 \mathrm{mg}, 0.02 \mathrm{mmol})$ were added. The flask was equipped with a reflux condenser and capped with a rubber septum. The apparatus was purged by three cycles of evacuation and $\mathrm{N}_{2}$ refill. Toluene $(12 \mathrm{~mL})$, ethanol $(4.8 \mathrm{~mL})$, and water $(2.4 \mathrm{~mL})$ were added via syringe and the mixture was allowed to stir under reflux in an oil bath. After 14 hours, the reaction vessel was allowed to cool to room temperature and water $(10 \mathrm{~mL})$ was added. The organics were extracted with EtOAc ( $3 \mathrm{x}, 10 \mathrm{~mL}$ each), dried over $\mathrm{Na}_{2} \mathrm{SO}_{4}$, and concentrated by rotary evaporator. The crude mixture was purified by silica column chromatography using 4:1 petroleum ether/ethyl acetate as the eluent afforded the product (white solid, $450 \mathrm{mg}$, $80 \%$ yield, $\left.\mathbf{M p} 122-123{ }^{\circ} \mathrm{C}\right) .{ }^{[4]}$

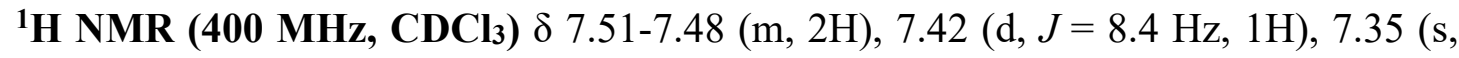
$1 \mathrm{H}), 7.06(\mathrm{~d}, J=8.4 \mathrm{~Hz}, 1 \mathrm{H}), 6.98-6.95(\mathrm{~m}, 2 \mathrm{H}), 4.02(\mathrm{q}, J=6.8 \mathrm{~Hz}, 2 \mathrm{H}), 3.85(\mathrm{~s}, 3 \mathrm{H})$, 2.96-2.92 (m, 2H), 2.69-2.65 (m, 2H), $1.28(\mathrm{t}, J=7.2 \mathrm{~Hz}, 3 \mathrm{H}) \mathrm{ppm} ;{ }^{13} \mathbf{C}$ NMR (101 MHz, $\left.\mathbf{C D C l}_{3}\right) \delta 169.7,159.0,138.3,135.3,132.8,127.7,126.8,126.3,125.5,114.9$, 114.2, 55.3, 37.4, 31.9, 25.8, 12.8 ppm; IR (neat) v 2948, 2910, 1678, 1660, 1242, 1185, 1021, $803 \mathrm{~cm}^{-1}$. HRMS (ESI) m/z: $[\mathrm{M}+\mathrm{H}]^{+}$calcd for $\mathrm{C}_{18} \mathrm{H}_{20} \mathrm{NO}_{2} 282.1489$; found: 282.1494 .

\section{7-(4-bromobutoxy)-1-ethyl-3,4-dihydroquinolin-2(1H)-one (1g)}

GP 2. 3 mmol-scale, purified by flash column chromatography (eluent: petroleum ether/ethyl acetate =4/1-1/1), pale yellow oil, $200 \mathrm{mg}, 20 \%$ yield. ${ }^{1} \mathbf{H}$ NMR (400 MHz, $\left.\mathbf{C D C l}_{3}\right) \delta 7.05(\mathrm{~d}, J=8.4 \mathrm{~Hz}, 1 \mathrm{H}), 6.58(\mathrm{~d}, J=2.4 \mathrm{~Hz}, 1 \mathrm{H}), 6.51(\mathrm{dd}, J=8.0,2.4 \mathrm{~Hz}$, $1 \mathrm{H}), 4.01-3.93(\mathrm{~m}, 4 \mathrm{H}), 3.50(\mathrm{t}, J=6.4 \mathrm{~Hz}, 2 \mathrm{H}), 2.83-2.80(\mathrm{~m}, 2 \mathrm{H}), 2.63-2.59(\mathrm{~m}, 2 \mathrm{H})$, 2.12-2.05 (m, 2H), 1.99-1.92 (m, 2H), $1.26(\mathrm{t}, J=7.2 \mathrm{~Hz}, 3 \mathrm{H}) \mathrm{ppm} ;{ }^{\mathbf{1 3}} \mathbf{C}$ NMR (101 MHz, CDCl $) \delta 170.0,158.4,140.5,128.4,118.8,106.8,102.9,67.0,37.4,33.4,32.2$, 29.4, 27.9, 24.8, 12.8 ppm; IR (neat) 2938, 2871, 1667, 1612, 1509, 1470, 1363, 1195, $1128,844 v \mathrm{~cm}^{-1}$. HRMS (ESI) m/z: $[\mathrm{M}+\mathrm{H}]^{+}$calcd for $\mathrm{C}_{15} \mathrm{H}_{21} \mathrm{NO}_{2} \mathrm{Br} 326.0750$; found: 326.0752 .

\section{6-bromo-1-ethyl-3,4-dihydro-1,8-naphthyridin-2(1H)-one (1h)}

GP 1. 3 mmol-scale, purified by flash column chromatography (eluent: petroleum ether/ethyl acetate $=4 / 1-1 / 1)$, white solid, $527 \mathrm{mg}, 70 \%$ yield. Mp 101-102 ${ }^{\circ} \mathrm{C} .{ }^{1} \mathbf{H}$ NMR (400 MHz, CDCl $\mathbf{l}_{3} \delta 8.28(\mathrm{~s}, 1 \mathrm{H}), 7.56(\mathrm{t}, J=1.2 \mathrm{~Hz}, 1 \mathrm{H}), 4.15$ (q, $J=7.2 \mathrm{~Hz}$, 2H), 2.89-2.86 (m, 2H), 2.70-2.66 (m, 2H), $1.21(\mathrm{t}, J=7.2 \mathrm{~Hz}, 3 \mathrm{H}) \mathrm{ppm} ;{ }^{13} \mathbf{C}$ NMR (101 MHz, CDCl3) $\delta 169.6,150.7,146.8,137.8,122.4,113.3,36.0,30.9,23.7,13.1$ ppm; IR (neat) $v$ 3051, 2970, 1657, 1373, 1198, 1136, 950, $687 \mathrm{~cm}^{-1}$. HRMS (ESI) $\mathbf{m} / \mathbf{z}:[\mathrm{M}+\mathrm{H}]^{+}$calcd for $\mathrm{C}_{10} \mathrm{H}_{12} \mathrm{~N}_{2} \mathrm{OBr} 255.0128$; found: 255.0132 . 


\section{1-(4-phenylbutyl)-3,4-dihydroquinolin-2(1H)-one(1j)}

GP 2. 5 mmol-scale, purified by flash column chromatography (eluent: petroleum ether/ethyl acetate $=4 / 1-1 / 1)$, pale yellow oil, $1216 \mathrm{mg}, 86 \%$ yield. ${ }^{1} \mathbf{H}$ NMR (400 MHz, $\left.\mathrm{CDCl}_{3}\right) \delta$ 7.29-7.25 (m, 2H), 7.24-7.14 (m, 5H), $6.99(\mathrm{~m}, 1 \mathrm{H}), 6.93(\mathrm{~d}, J=8.4 \mathrm{~Hz}, 1 \mathrm{H})$, $3.95(\mathrm{t}, J=7.2 \mathrm{~Hz}, 2 \mathrm{H}), 2.89-2.86(\mathrm{~m}, 2 \mathrm{H}), 2.68-2.61(\mathrm{~m}, 4 \mathrm{H}), 1.72-1.68(\mathrm{~m} 4 \mathrm{H}) \mathrm{ppm}$; ${ }^{13}$ C NMR (101 MHz, CDCl 3$) \delta 170.1,142.0,139.5,128.34,128.26,127.9,127.3$, 126.6, 125.7, 122.6, 114.8, 41.8, 35.4, 31.9, 28.6, 26.7, 25.5 ppm; IR (neat) v 3025, 2934, 1666, 1600, 1494, 1460, 1375, 1154, 750, 735, $698 \mathrm{~cm}^{-1}$. HRMS (ESI) m/z: [M $+\mathrm{H}]^{+}$calcd for $\mathrm{C}_{19} \mathrm{H}_{22} \mathrm{NO} 280.1696$; found: 280.1700 .

phenyl 2-(2-oxo-3,4-dihydroquinolin-1(2H)-yl)acetate (11)

GP 1. 10 mmol-scale, purified by flash column chromatography (eluent: petroleum ether/ethyl acetate =4/1-1/1), white solid, $1297 \mathrm{mg}$, 46\% yield. Mp 67-68 ${ }^{\circ} \mathrm{C} .{ }^{1} \mathbf{H}$ NMR (400 MHz, CDCl $) \delta$ 7.37-7.33 (m, 2H), 7.28-7.19 (m, 3H), 7.10-7.03 (m, 3H), 6.89 $(\mathrm{d}, J=8.2 \mathrm{~Hz}, 1 \mathrm{H}), 4.92(\mathrm{~s}, 2 \mathrm{H}), 2.98-2.95(\mathrm{~m}, 2 \mathrm{H}), 2.76-2.72(\mathrm{~m}, 2 \mathrm{H}) \mathrm{ppm} ;{ }^{13} \mathbf{C} \mathbf{N M R}$ (101 MHz, CDCl $) \delta 170.6,167.3,150.3,139.4,129.4,128.1,127.6,126.3,126.0$, 123.3, 121.2, 114.2, 44.4, 31.4, 25.3 ppm; IR (neat) v 2946, 2850, 1762, 1665, 1369, 1188, 1170, 1152, 1024, 753, $687 \mathrm{~cm}^{-1}$. HRMS (ESI) m/z: $[\mathrm{M}+\mathrm{Na}]^{+}$calcd for $\mathrm{C}_{17} \mathrm{H}_{15} \mathrm{NO}_{3} \mathrm{Na}$ 304.0944; found: 304.0947 .

\section{1-((1,3-dioxolan-2-yl)methyl)-3,4-dihydroquinolin-2(1H)-one (1 m)}

GP 2. 5 mmol-scale, purified by flash column chromatography (eluent: petroleum ether/ethyl acetate $=4 / 1-1 / 1)$, white solid, $793 \mathrm{mg}, 68 \%$ yield. Mp 73-74 ${ }^{\circ} \mathrm{C}$. ${ }^{\mathbf{1}} \mathbf{H}$ NMR

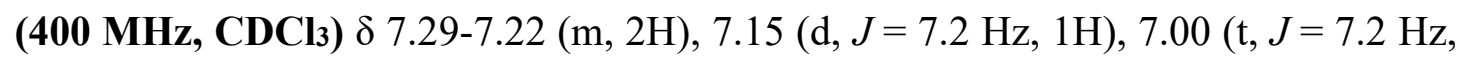
$1 \mathrm{H}), 5.18-5.15(\mathrm{~m}, 1 \mathrm{H}), 4.15(\mathrm{~d}, J=4.4 \mathrm{~Hz}, 2 \mathrm{H}), 4.05-4.01(\mathrm{~m}, 2 \mathrm{H}), 3.89-3.86(\mathrm{~m}, 2 \mathrm{H})$, $2.91(\mathrm{t}, J=7.2 \mathrm{~Hz}, 2 \mathrm{H}), 2.67(\mathrm{t}, J=7.2 \mathrm{~Hz}, 2 \mathrm{H}) \mathrm{ppm} ;{ }^{13} \mathbf{C} \mathbf{N M R}\left(\mathbf{1 0 1} \mathbf{~ M H z}, \mathbf{C D C l}_{3}\right) \delta$ $170.6,140.2,127.8,127.3,126.4,122.9,115.7,101.7,64.9,45.5,31.8,25.5$ ppm; IR (neat) $v$ 2952, 2913, 1676, 1482, 1242, 1210, 1030, $750 \mathrm{~cm}^{-1}$. HRMS (ESI) m/z: [M + $\mathrm{Na}]^{+}$calcd for $\mathrm{C}_{13} \mathrm{H}_{15} \mathrm{NO}_{3} \mathrm{Na} 256.0944$; found: 256.0951 .

\section{1-(2-((tetrahydro-2H-pyran-2-yl)oxy)ethyl)-3,4-dihydroquinolin-2(1H)-one (10)}

GP 2. 5 mmol-scale, purified by flash column chromatography (eluent: petroleum ether/ethyl acetate $=4 / 1-1 / 1)$, pale yellow oil, $827 \mathrm{mg}, 60 \%$ yield. ${ }^{1} \mathbf{H}$ NMR (400 MHz, $\left.\mathbf{C D C l}_{3}\right) \delta 7.28-7.21(\mathrm{~m}, 2 \mathrm{H}), 7.15(\mathrm{~d}, J=7.2 \mathrm{~Hz}, 1 \mathrm{H}), 7.00(\mathrm{td}, J=7.2,1.6 \mathrm{~Hz}, 1 \mathrm{H})$, $4.61(\mathrm{t}, J=3.2 \mathrm{~Hz}, 1 \mathrm{H}), 4.16(\mathrm{t}, J=6.4 \mathrm{~Hz}, 2 \mathrm{H}), 3.98-3.93(\mathrm{~m}, 1 \mathrm{H}), 3.80-3.75(\mathrm{~m}, 1 \mathrm{H})$, 3.72-3.66 (m, $1 \mathrm{H}), 3.50-3.45(\mathrm{~m}, 1 \mathrm{H}), 2.91-2.88(\mathrm{~m}, 2 \mathrm{H}), 2.67-2.63(\mathrm{~m}, 2 \mathrm{H}), 1.70-1.64$ (m, 2H), 1.58-1.47 (m, 4H) ppm; ${ }^{13} \mathbf{C}$ NMR (101 MHz, CDCl $) \delta 170.4,140.2,127.7$, $127.3,126.5,122.8,115.8,98.8,64.4,62.0,42.9,31.9,30.4,25.6,25.3,19.2$ ppm; IR (neat) $v$ 2940, 2870, 1668, 1372, 1120, 1031, $753 \mathrm{~cm}^{-1}$. HRMS (ESI) m/z: $[\mathrm{M}+\mathrm{Na}]^{+}$ calcd for $\mathrm{C}_{16} \mathrm{H}_{21} \mathrm{NO}_{3} \mathrm{Na} 298.1413$; found: 298.1422.

\section{ethyl 4-(2-oxo-3,4-dihydroquinolin-1(2H)-yl)butanoate (1p)}

GP 2. 5 mmol-scale, purified by flash column chromatography (eluent: petroleum ether/ethyl acetate =4/1-1/1), colorless oil, $651 \mathrm{mg}, 50 \%$ yield. ${ }^{1} \mathbf{H}$ NMR (400 MHz, 
$\left.\mathbf{C D C l}_{3}\right) \delta$ 7.29-7.24 (m, 1H), 7.17-7.13 (m, 2H), $7.01(\mathrm{td}, J=7.2,1.2 \mathrm{~Hz}, 1 \mathrm{H}), 4.15(\mathrm{q}$, $J=7.2 \mathrm{~Hz}, 2 \mathrm{H}), 4.00-3.96(\mathrm{~m}, 2 \mathrm{H}), 2.91-2.88(\mathrm{~m}, 2 \mathrm{H}), 2.66-2.62(\mathrm{~m}, 2 \mathrm{H}), 2.41(\mathrm{t}, J=$ $7.2 \mathrm{~Hz}, 2 \mathrm{H}), 2.01-1.94(\mathrm{~m}, 2 \mathrm{H}), 1.27$ (t, $J=7.2 \mathrm{~Hz}, 3 \mathrm{H}) \mathrm{ppm} ;{ }^{13} \mathbf{C}$ NMR (101 MHz, $\left.\mathbf{C D C l}_{3}\right) \delta 173.0,170.2,139.4,127.9,127.5,126.3,122.7,114.8,60.5,41.4,31.8,31.4$, 25.5, 22.2, 14.2 ppm; IR (neat) v 2979, 2905, 1728, 1666, 1600, 1496, 1460, 1374, $1161,753 \mathrm{~cm}^{-1}$. HRMS (ESI) m/z: $[\mathrm{M}+\mathrm{Na}]^{+}$calcd for $\mathrm{C}_{15} \mathrm{H}_{19} \mathrm{NO}_{3} \mathrm{Na} 284.1257$; found: 284.1260 .

\section{1-(benzo[d][1,3]dioxol-5-yl)-1,2,3,4-tetrahydroquinoline (1s)}

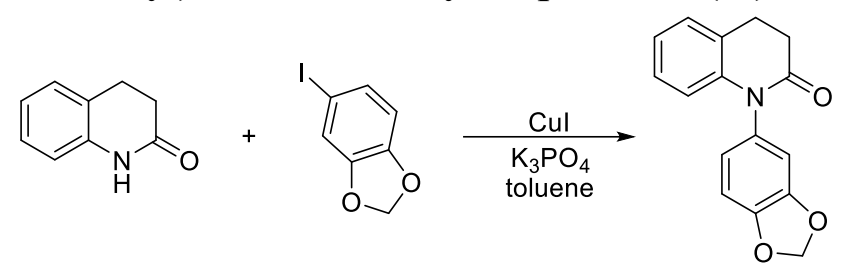

After three cycles of evacuation and backfilling with dry nitrogen, an oven-dried Schlenk tube equipped with a magnetic stirring bar was charged with 3,4dihydroquinolin-2(1H)-one ( $882 \mathrm{mg}, 6 \mathrm{mmol}), \mathrm{CuI}(38 \mathrm{mg}, 0.2 \mathrm{mmol}), \mathrm{K}_{3} \mathrm{PO}_{4}(2.12 \mathrm{~g}$, $10 \mathrm{mmol})$, and 5-iodobenzo[d][1,3]dioxole $(5 \mathrm{mmol})$. The tube was evacuated and backfilled with nitrogen, and then 2,2,6,6-tetramethyl-3,5-heptanedione (92 mg, 0.5 $\mathrm{mmol})$ and anhydrous degassed toluene $(5 \mathrm{~mL})$ were added under a stream of nitrogen by syringe at room temperature. The tube was sealed under a positive pressure of nitrogen, stirred, and heated to $130{ }^{\circ} \mathrm{C}$ in an oil bath for $24 \mathrm{~h}$. After being cooled to room temperature, the reaction was diluted with $100 \mathrm{~mL}$ of $\mathrm{CH}_{2} \mathrm{Cl}_{2}$ and washed twice with water. The aqueous phases were extracted five times with dichloromethane. The organic layers were combined, dried over $\mathrm{MgSO}_{4}$, filtered, and concentrated under vacuum. Purification of the residue by chromatography on silica gel using 2:1 petroleum ether / EtOAc as the eluent afforded the product (white solid, 886mg, 66\% yield, Mp $\left.170-171^{\circ} \mathrm{C}\right) .^{[5]}$

${ }^{1} \mathbf{H}$ NMR (400 MHz, CDCl3) $\delta 7.19(\mathrm{~d}, J=7.3 \mathrm{~Hz}, 1 \mathrm{H}), 7.07(\mathrm{td}, J=7.6,1.6 \mathrm{~Hz}, 1 \mathrm{H})$, $6.98(\mathrm{td}, J=7.2,1.2 \mathrm{~Hz}, 1 \mathrm{H}), 6.92-6.89(\mathrm{~m}, 1 \mathrm{H}), 6.70-6.68(\mathrm{~m}, 2 \mathrm{H}), 6.46(\mathrm{dd}, J=8.0$, $1.2 \mathrm{~Hz}, 1 \mathrm{H}), 6.04$ (s, 2H), 3.07-3.03 (m, 2H), 2.83-2.79 (m, 2H) ppm; ${ }^{13}$ C NMR (101 MHz, $\left.\mathbf{C D C l}_{3}\right) \delta 170.4,148.6,147.4,141.6,131.8,127.7,127.1,125.4,122.9,122.2$, 116.8, 109.8, 108.9, 101.7, 32.1, 25.5 ppm. IR (neat) v 2961, 2881, 1667, 1451, 1376, 1130, 1039, $763 \mathrm{~cm}^{-1}$. HRMS (ESI) m/z: $[\mathrm{M}+\mathrm{H}]^{+}$calcd for $\mathrm{C}_{16} \mathrm{H}_{14} \mathrm{NO}_{3} 268.0968$; found: 268.0972 .

\section{6-(4-(1-cyclohexyl-1H-tetrazol-5-yl)butoxy)-1-ethyl-3,4-dihydroquinolin-2(1H)- one (5d)}

GP 1. 5 mmol-scale, purified by flash column chromatography (eluent: petroleum ether/ethyl acetate $=4 / 1-1 / 1)$, pale yellow oil, $1.60 \mathrm{~g}, 81 \%$ yield. ${ }^{1} \mathbf{H}$ NMR (400 MHz, CDCl $) \delta 6.92(\mathrm{~d}, J=8.8 \mathrm{~Hz}, 1 \mathrm{H}), 6.75(\mathrm{dd}, J=8.8,2.8 \mathrm{~Hz}, 1 \mathrm{H}), 6.71(\mathrm{~d}, J=2.8 \mathrm{~Hz}$, $1 \mathrm{H}), 4.17-4.09(\mathrm{~m}, 1 \mathrm{H}), 4.01-3.93(\mathrm{~m}, 4 \mathrm{H}), 2.93(\mathrm{t}, J=7.6 \mathrm{~Hz}, 2 \mathrm{H}), 2.86-2.82(\mathrm{~m}, 2 \mathrm{H})$, 2.63-2.59 (m, 2H), 2.08-1.76 (m, 12H), 1.44-1.32 (m, 2H), $1.24(\mathrm{t}, J=7.2 \mathrm{~Hz}, 3 \mathrm{H}) \mathrm{ppm}$; ${ }^{13}$ C NMR (101 MHz, CDCl 3$) \delta 169.4,154.3,153.5,133.1,128.1,115.5,114.5,112.6$, 
$67.4,57.6,37.3,32.9,31.8,28.5,25.8,25.3,24.8,24.0,23.0,12.8$ ppm; IR (neat) $v$ 2935, 2860, 1656, 1502, 1440. 1385, 1233, $750 \mathrm{~cm}^{-1}$. HRMS (ESI) m/z: calcd for $\left[\mathrm{C}_{22} \mathrm{H}_{32} \mathrm{~N}_{5} \mathrm{O}_{2}\right]^{+}\left([\mathrm{M}+\mathrm{H}]^{+}\right): 398.2551$; found: 398.2553 .

\section{6-(4-(4-(2,3-dichlorophenyl)piperazin-1-yl)butoxy)-1-ethyl-3,4-dihydroquinolin- 2(1H)-one (5e)}

GP 1. 5 mmol-scale, purified by flash column chromatography (eluent: petroleum ether/ethyl acetate $=4 / 1-1 / 1)$, white solid, $1911 \mathrm{mg}$, 80\% yield. Mp 135-136 ${ }^{\circ} \mathrm{C} .{ }^{1} \mathbf{H}$ NMR (400 MHz, CDCl $) \delta$ 7.15-7.14 (m, 2H), 7.04 (d, $J=8.4 \mathrm{~Hz}, 1 \mathrm{H}), 6.95$ (dd, $J=$ 6.4, $3.2 \mathrm{~Hz}, 1 \mathrm{H}), 6.59(\mathrm{~d}, J=2.4 \mathrm{~Hz}, 1 \mathrm{H}), 6.52(\mathrm{dd}, J=8.0,2.4 \mathrm{~Hz}, 1 \mathrm{H}), 4.01-3.93(\mathrm{~m}$, $4 \mathrm{H}), 3.08-3.06(\mathrm{~m}, 4 \mathrm{H}), 2.83-2.79(\mathrm{~m}, 2 \mathrm{H}), 2.66-2.59(\mathrm{~m}, 6 \mathrm{H}), 2.52-2.48(\mathrm{~m}, 2 \mathrm{H}), 1.89-$ 1.81(m, 2H), 1.76-1.69 (m, 2H), 1.26 (t, $J=7.2 \mathrm{~Hz}, 3 \mathrm{H}) \mathrm{ppm} ;{ }^{13} \mathbf{C}$ NMR (101 MHz, $\left.\mathbf{C D C l}_{3}\right) \delta 169.9,158.5,151.2,140.4,133.9,128.3,127.4,124.43,124.42,118.5,106.8$, $102.8,67.8,58.1,53.2,51.2,37.4,32.2,27.2,24.7,23.4,12.7 \mathrm{ppm}$ (One carbon is missing because of overlapping); IR (neat) $v$ 2942, 2803, 1669, 1612, 1586, 1442, 1361, 1201, 1127, $779 \mathrm{~cm}^{-1}$. HRMS (ESI) m/z: $[\mathrm{M}+\mathrm{H}]^{+}$calcd for $\mathrm{C}_{25} \mathrm{H}_{32} \mathrm{~N}_{3} \mathrm{O}_{2} \mathrm{Cl}_{2} 476.1866$; found: 476.1862 .

\section{Catalytic reactions}

\subsection{General procedure<smiles>[R]N1C(=O)CCc2ccccc21</smiles> \\ (1) $\mathrm{IrCl}(\mathrm{CO})\left(\mathrm{PPh}_{3}\right)_{2}(1 \mathrm{~mol} \%)$

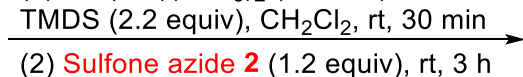 \\ $1 \mathrm{R}$

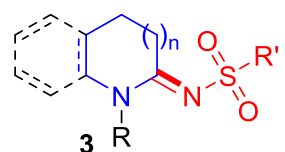

To a solution of $\operatorname{IrCl}(\mathrm{CO})\left(\mathrm{PPh}_{3}\right)_{2}$ in DCM $(1 \mathrm{~mL}, 0.002 \mathrm{mmol}$ of [Ir] $)$ was added the lactam $(0.2 \mathrm{mmol})$ and TMDS (59 $\mathrm{mg}, 0.44 \mathrm{mmol})$. The solution was stirred for $30 \mathrm{~min}$ at RT. The sulfone azide $(0.24 \mathrm{mmol})$ was added and stirred at RT for $3 \mathrm{~h}$. The solvent was removed under reduced pressure and the residue was purified by flash chromatography on silica gel using a mixture of ethyl acetate/petroleum ether as eluent to yield amidines.

\section{(E)- $N$-(1-ethyl-3,4-dihydroquinolin-2(1H)-ylidene)-4-methylbenzenesulfonamide}

(3a)

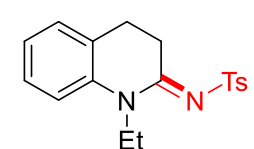

Purified by flash column chromatography (eluent: petroleum ether/ethyl acetate =10/1-1/1), white solid, $53 \mathrm{mg}, 80 \%$ yield. Mp 166$167{ }^{\circ} \mathrm{C} .{ }^{\mathbf{1}} \mathbf{H}$ NMR (400 MHz, $\left.\mathbf{C D C l}_{3}\right): \delta 7.86(\mathrm{~d}, J=8.0 \mathrm{~Hz}, 2 \mathrm{H}), 7.30$ $7.24(\mathrm{~m}, 3 \mathrm{H}), 7.17$ (d, $J=7.2 \mathrm{~Hz}, 1 \mathrm{H}), 7.11-7.06(\mathrm{~m}, 2 \mathrm{H}), 4.06(\mathrm{q}, J=7.2 \mathrm{~Hz}, 2 \mathrm{H}), 3.30$ (t, $J=7.2 \mathrm{~Hz}, 2 \mathrm{H}), 2.83(\mathrm{t}, J=7.2 \mathrm{~Hz}, 2 \mathrm{H}), 2.42(\mathrm{~s}, 3 \mathrm{H}), 1.29$ (t, $J=7.2 \mathrm{~Hz}, 3 \mathrm{H}) \mathrm{ppm}$; ${ }^{13}$ C NMR (101 MHz, $\left.\mathbf{C D C l}_{3}\right): \delta 164.8,142.2,140.6,138.2,129.2,127.9,127.7,127.2$, 126.2, 124.2, 115.8, 41.1, 27.8, 23.9, 21.4, 12.1 ppm; IR (neat) v 2944, 1577, 1263, 1130, 1081, 811, 756, $680 \mathrm{~cm}^{-1}$. HRMS (ESI) m/z: $[\mathrm{M}+\mathrm{H}]^{+}$calcd for $\mathrm{C}_{18} \mathrm{H}_{21} \mathrm{~N}_{2} \mathrm{O}_{2} \mathrm{~S}$ 329.1318 ; found 329.1327 . 
(E)- $N$-(6-bromo-1-ethyl-3,4-dihydroquinolin-2(1H)-ylidene)-4methylbenzenesulfonamide (3b)

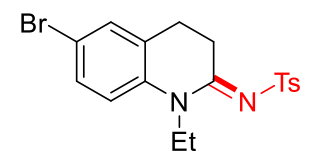

Purified by flash column chromatography (eluent: petroleum ether/ethyl acetate =10/1-1/1), white solid, $61 \mathrm{mg}, 75 \%$ yield. $\mathbf{M p}$ 172-173 ${ }^{\circ} \mathrm{C} .{ }^{1} \mathbf{H}$ NMR $\left(400 \mathbf{~ M H z}, \mathbf{C D C l}_{3}\right) \delta 7.85(\mathrm{~d}, J=8.0 \mathrm{~Hz}$, $2 \mathrm{H}), 7.37(\mathrm{dd}, J=8.4,2.4 \mathrm{~Hz}, 1 \mathrm{H}), 7.31-7.27(\mathrm{~m}, 3 \mathrm{H}), 6.96(\mathrm{~d}, J=8.8 \mathrm{~Hz}, 1 \mathrm{H}), 4.02$ (q, $J=7.2 \mathrm{~Hz}, 2 \mathrm{H}), 3.32-3.28(\mathrm{~m}, 2 \mathrm{H}), 2.83-2.8(\mathrm{~m}, 2 \mathrm{H}), 2.42(\mathrm{~s}, 3 \mathrm{H}), 1.26(\mathrm{t}, J=7.2 \mathrm{~Hz}$, 3H) ppm; ${ }^{13}$ C NMR (101 MHz, CDCl3) $\delta$ 164.3, 142.4, 140.4, 137.4, 130.7, 130.6, $129.2,126.2,117.4,117.0,41.1,27.6,23.7,21.5,12.0 \mathrm{ppm}$ (One carbon is missing because of overlapping); IR (neat) v 2911, 2852, 1598, 1437, 1265, 1134, 1082, 806, 760, $680 \mathrm{~cm}^{-1}$. HRMS (ESI) m/z: $[\mathrm{M}+\mathrm{H}]^{+}$calcd for $\mathrm{C}_{18} \mathrm{H}_{20} \mathrm{~N}_{2} \mathrm{O}_{2} \mathrm{~S} 407.0423$; found: 407.0414 .

\section{(E)- $N$-(5-bromo-1-ethyl-3,4-dihydroquinolin-2(1H)-ylidene)-4-} methylbenzenesulfonamide $(3 \mathrm{c})$

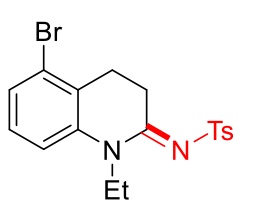

Purified by flash column chromatography (eluent: petroleum ether/ethyl acetate =10/1-1/1), white solid, $66 \mathrm{mg}$, 81\% yield. Mp 173$174{ }^{\circ} \mathrm{C} .{ }^{\mathbf{1}} \mathbf{H}$ NMR (400 MHz, CDCl 3$) \delta 7.85(\mathrm{~d}, J=8.4 \mathrm{~Hz}, 2 \mathrm{H}), 7.33-$ $7.27(\mathrm{~m}, 3 \mathrm{H}), 7.13(\mathrm{t}, J=8.4 \mathrm{~Hz}, 1 \mathrm{H}), 7.06(\mathrm{~d}, J=8.0 \mathrm{~Hz}, 1 \mathrm{H}), 4.04$ (q, $J=7.2 \mathrm{~Hz}, 2 \mathrm{H}), 3.30-3.26(\mathrm{~m}, J=8.6,6.3 \mathrm{~Hz}, 2 \mathrm{H}), 2.97-2.93(\mathrm{~m}, 2 \mathrm{H}), 2.42(\mathrm{~s}, 3 \mathrm{H})$, 1.27 (t, $J=7.2 \mathrm{~Hz}, 3 \mathrm{H}) \mathrm{ppm} ;{ }^{13} \mathbf{C}$ NMR (101 MHz, CDCl $) \delta 164.4,142.4,140.3$, 139.7, 129.2, 128.4, 128.2, 127.2, 126.2, 123.4, 115.1, 41.5, 27.1, 23.8, 21.4, 12.1 ppm; IR (neat) v 2919, 2848, 1535, 1264, 1133, 1081, 811, 776, $691 \mathrm{~cm}^{-1}$. HRMS (ESI) m/z: $[\mathrm{M}+\mathrm{Na}]^{+}$calcd for $\mathrm{C}_{18} \mathrm{H}_{19} \mathrm{~N}_{2} \mathrm{O}_{2} \mathrm{NaSBr} 429.0243$; found: 429.0243 .

(E)-N-(7-chloro-1-ethyl-3,4-dihydroquinolin-2(1H)-ylidene)-4methylbenzenesulfonamide (3d)

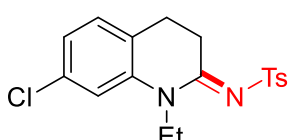

Purified by flash column chromatography (eluent: petroleum ether/ethyl acetate $=10 / 1-1 / 1)$, white solid, $51 \mathrm{mg}, 70 \%$ yield. Mp 143-144 ${ }^{\circ} \mathrm{C} .{ }^{1} \mathbf{H}$ NMR (400 MHz, CDCl $) \delta 7.85(\mathrm{~d}, J=8.0 \mathrm{~Hz}$, $2 \mathrm{H}), 7.29(\mathrm{~d}, J=8.0 \mathrm{~Hz}, 2 \mathrm{H}), 7.11-7.03(\mathrm{~m}, 3 \mathrm{H}), 4.01(\mathrm{t}, J=7.2 \mathrm{~Hz}, 2 \mathrm{H}), 3.32-3.28(\mathrm{~m}$, 2H), 2.80 (t, $J=7.2 \mathrm{~Hz}, 2 \mathrm{H}), 2.42(\mathrm{~s}, 3 \mathrm{H}), 1.27$ (t, $J=7.2 \mathrm{~Hz}, 3 \mathrm{H}) \mathrm{ppm} ;{ }^{13} \mathbf{C}$ NMR (101 MHz, CDCl 3$) \delta 164.6,142.4,140.4,139.4,133.3,129.2,128.8,126.3,125.4,123.9$, 116.2, 41.2, 27.7, 23.5, 21.5, 12.0 ppm; IR (neat) v 2983, 2917, 1580, 1437, 1275, 1145 , 1083, 843, $682 \mathrm{~cm}^{-1}$. HRMS (ESI) $\mathbf{m} / \mathbf{z}:[\mathrm{M}+\mathrm{Na}]^{+}$calcd for $\mathrm{C}_{18} \mathrm{H}_{19} \mathrm{~N}_{2} \mathrm{O}_{2} \mathrm{NaSCl}$ 385.0748; found: 385.0739 .

\section{(E)- $N$-(1-ethyl-6-methoxy-3,4-dihydroquinolin-2(1H)-ylidene)-4-} methylbenzenesulfonamide ( $3 \mathrm{e})$

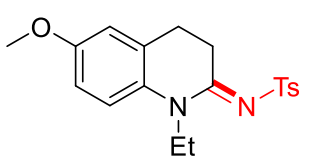

Purified by flash column chromatography (eluent: petroleum ether/ethyl acetate =10/1-1/1), white solid, $61 \mathrm{mg}, 85 \%$ yield. $\mathbf{M p}$ 172-173 ${ }^{\circ} \mathrm{C} .{ }^{1} \mathbf{H}$ NMR (400 MHz, CDCl 3$) \delta 7.86(\mathrm{~d}, J=8.0 \mathrm{~Hz}$, $2 \mathrm{H}), 7.29-7.27(\mathrm{~m}, 2 \mathrm{H}), 7.02(\mathrm{~d}, J=8.8 \mathrm{~Hz}, 1 \mathrm{H}), 6.78(\mathrm{dd}, J=8.8$, $3.2 \mathrm{~Hz}, 1 \mathrm{H}), 6.72(\mathrm{~d}, J=2.8 \mathrm{~Hz}, 1 \mathrm{H}), 4.04(\mathrm{q}, J=7.2 \mathrm{~Hz}, 2 \mathrm{H}), 3.79$ (s, 3H), 3.28-3.25 
(m, 2H), $2.79(\mathrm{t}, J=7.2 \mathrm{~Hz}, 2 \mathrm{H}), 2.41(\mathrm{~s}, 3 \mathrm{H}), 1.27(\mathrm{t}, J=7.2 \mathrm{~Hz}, 3 \mathrm{H}) \mathrm{ppm} ;{ }^{13} \mathbf{C} \mathbf{~ N M R}$ (101 MHz, CDCl3) $\delta 164.1,156.2,142.1,140.8,131.6,129.2,128.8,126.2,117.0$, 113.6, 112.4, 55.5, 41.2, 27.8, 24.3, 21.5, 12.2 ppm; IR (neat) v 2918, 2848, 1501, 1267 , 1138, 1080, 812, $753 \mathrm{~cm}^{-1}$. HRMS (ESI) m/z: $[\mathrm{M}+\mathrm{Na}]^{+}$calcd for $\mathrm{C}_{19} \mathrm{H}_{22} \mathrm{~N}_{2} \mathrm{O}_{3} \mathrm{NaS}$ 381.1243; found: 381.1240 .

(E)-N-(1-ethyl-6-(4-methoxyphenyl)-3,4-dihydroquinolin-2(1H)-ylidene)-4methylbenzenesulfonamide (3f)

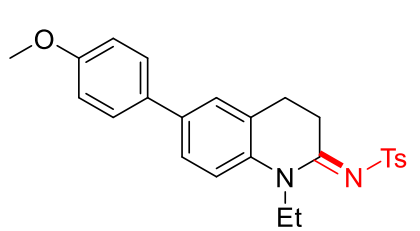

Purified by flash column chromatography (eluent: petroleum ether/ethyl acetate $=10 / 1-1 / 1)$, pale yellow solid, $74 \mathrm{mg}, 85 \%$ yield. Mp 208-209 ${ }^{\circ} \mathrm{C} .{ }^{1} \mathbf{H}$ NMR (400 MHz, CDCl 3$) \delta 7.87(\mathrm{~d}, J=8.4 \mathrm{~Hz}, 2 \mathrm{H}), 7.48(\mathrm{~d}, J=8.8 \mathrm{~Hz}, 2 \mathrm{H})$, $7.43(\mathrm{dd}, J=8.4,2.8 \mathrm{~Hz}, 1 \mathrm{H}), 7.34(\mathrm{~d}, J=2.0 \mathrm{~Hz}, 1 \mathrm{H}), 7.28$ $(\mathrm{d}, J=8.0 \mathrm{~Hz}, 2 \mathrm{H}), 7.12(\mathrm{~d}, J=8.4 \mathrm{~Hz}, 1 \mathrm{H}), 6.96(\mathrm{~d}, J=8.4 \mathrm{~Hz}, 2 \mathrm{H}), 4.08(\mathrm{q}, J=7.2$ $\mathrm{Hz}, 2 \mathrm{H}), 3.83(\mathrm{~s}, 3 \mathrm{H}), 3.33-3.30(\mathrm{~m}, 2 \mathrm{H}), 2.86(\mathrm{t}, J=7.2 \mathrm{~Hz}, 2 \mathrm{H}), 2.40(\mathrm{~s}, 3 \mathrm{H}), 1.30(\mathrm{t}$, $J=7.2 \mathrm{~Hz}, 3 \mathrm{H}) \mathrm{ppm} ;{ }^{13} \mathbf{C}$ NMR (101 MHz, CDCl$) \delta 164.5,159.2,142.2,140.6,136.8$, 136.7, 132.2, 129.2, 127.7, 127.4, 126.2, 126.0, 125.6, 116.2, 114.2, 55.3, 41.0, 27.8, 24.0, 21.4, 12.1 ppm; IR (neat) v 2919, 2850, 1607, 1533, 1492, 1272, 1134, 1080, 810, $690 \mathrm{~cm}^{-1}$. HRMS (ESI) m/z: $[\mathrm{M}+\mathrm{H}]^{+}$calcd for $\mathrm{C}_{25} \mathrm{H}_{27} \mathrm{~N}_{2} \mathrm{O}_{3} \mathrm{~S}$ 435.1737; found: 435.1734 .

\section{(E)-N-(7-(4-bromobutoxy)-1-ethyl-3,4-dihydroquinolin-2(1H)-ylidene)-4-} methylbenzenesulfonamide $(3 \mathrm{~g})$

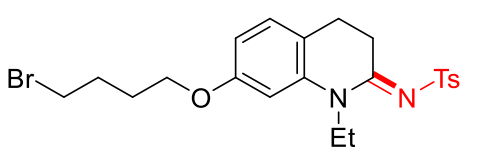

Purified by flash column chromatography (eluent: petroleum ether/ethyl acetate $=10 / 1-1 / 1)$, white solid, 62 mg, 65\% yield. Mp 124-125 ${ }^{\circ} \mathrm{C} .{ }^{\mathbf{1}} \mathbf{H}$ NMR (400 MHz, $\left.\mathbf{C D C l}_{3}\right) \delta 7.86(\mathrm{~d}, J=8.0 \mathrm{~Hz}, 2 \mathrm{H}), 7.28(\mathrm{~d}, J=8.4 \mathrm{~Hz}, 2 \mathrm{H}), 7.05(\mathrm{~d}, J=8.4 \mathrm{~Hz}, 1 \mathrm{H})$, $6.65(\mathrm{~d}, J=2.0 \mathrm{~Hz}, 1 \mathrm{H}), 6.58(\mathrm{dd}, J=8.4,2.4 \mathrm{~Hz}, 1 \mathrm{H}), 4.05-3.96(\mathrm{~m}, 4 \mathrm{H}), 3.48(\mathrm{t}, J=$ $6.8 \mathrm{~Hz}, 2 \mathrm{H}), 3.29-3.25(\mathrm{~m}, 2 \mathrm{H}), 2.75(\mathrm{t}, J=7.2 \mathrm{k} \mathrm{Hz}, 2 \mathrm{H}), 2.41(\mathrm{~s}, 3 \mathrm{H}), 2.11-2.03(\mathrm{~m}$, 2H), 1.98-1.91 (m, 2H), $1.28(\mathrm{t}, J=7.2 \mathrm{~Hz}, 3 \mathrm{H}) \mathrm{ppm} ;{ }^{13} \mathbf{C}$ NMR (101 MHz, CDCl 3$) \delta$ $164.9,158.5,142.2,140.6,139.1,129.2,128.4,126.23,119.3,108.5,104.2,67.1,41.2$, 33.3, 29.3, 28.2, 27.2, 23.1, 21.4, $12.1 \mathrm{ppm}$; IR (neat) v 2920, 2874, 1620, 1271, 1135, 1084, 900, $683 \mathrm{~cm}^{-1}$. HRMS (ESI) $\mathbf{m} / \mathbf{z}:[\mathrm{M}+\mathrm{H}]^{+}$calcd for $\mathrm{C}_{22} \mathrm{H}_{28} \mathrm{~N}_{2} \mathrm{O}_{3} \mathrm{SBr} 479.0998$; found: 479.0995 .

\section{(E)- $N$-(6-bromo-1-ethyl-3,4-dihydro-1,8-naphthyridin-2(1H)-ylidene)-4-} methylbenzenesulfonamide $(3 \mathrm{~h})$

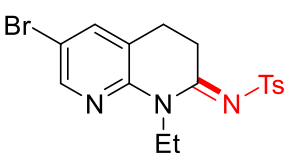

Purified by flash column chromatography (eluent: petroleum ether/ethyl acetate $=10 / 1-1 / 1)$, white solid, $62 \mathrm{mg}, 76 \%$ yield. $\mathbf{M p}$ 153-154 ${ }^{\circ} \mathrm{C} .{ }^{1} \mathbf{H}$ NMR (400 MHz, $\left.\mathbf{C D C l}_{3}\right) \delta 8.30$ (s, 1H), $7.86(\mathrm{~d}$, $J=8.0 \mathrm{~Hz}, 2 \mathrm{H}), 7.59(\mathrm{~s}, 1 \mathrm{H}), 7.30(\mathrm{~d}, J=8.0 \mathrm{~Hz}, 2 \mathrm{H}), 4.24(\mathrm{q}, J=7.2 \mathrm{~Hz}, 2 \mathrm{H}), 3.39(\mathrm{t}$, $J=7.8 \mathrm{~Hz}, 2 \mathrm{H}), 2.87(\mathrm{t}, J=7.8 \mathrm{~Hz}, 2 \mathrm{H}), 2.43(\mathrm{~s}, 3 \mathrm{H}), 1.19(\mathrm{t}, J=7.2 \mathrm{~Hz}, 3 \mathrm{H}) \mathrm{ppm} ;{ }^{13} \mathrm{C}$ NMR (101 MHz, CDCl $) \delta 164.5,149.1,147.2,142.6,140.2,138.1,129.3,126.3$, 122.9, 114.9, 39.1, 27.0, 22.7, 21.5, 12.3 ppm; IR (neat) $v$ 2915, 1553, 1432, 1264, 
1133, 1084, 859, 748, $678 \mathrm{~cm}^{-1}$. HRMS (ESI) m/z: $[\mathrm{M}+\mathrm{H}]^{+}$calcd for $\mathrm{C}_{17} \mathrm{H}_{19} \mathrm{~N}_{3} \mathrm{O}_{2} \mathrm{SBr}$ 408.0376; found: 408.0372 .

\section{(E)-N-(1-benzyl-3,4-dihydroquinolin-2(1H)-ylidene)-4-} methylbenzenesulfonamide (3i)

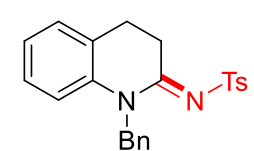

Purified by flash column chromatography (eluent: petroleum ether/ethyl acetate =10/1-1/1), white solid, $66 \mathrm{mg}, 84 \%$ yield. Mp 140$141{ }^{\circ} \mathrm{C} .{ }^{1} \mathbf{H}$ NMR $\left(400 \mathbf{M H z}, \mathbf{C D C l}_{3}\right) \delta 7.61(\mathrm{~d}, J=8.0 \mathrm{~Hz}, 2 \mathrm{H}), 7.30-$ $7.25(\mathrm{~m}, 3 \mathrm{H}), 7.19-7.12(\mathrm{~m}, 6 \mathrm{H}), 7.07-7.02(\mathrm{~m}, 1 \mathrm{H}), 6.97(\mathrm{~d}, J=8.0 \mathrm{~Hz}, 1 \mathrm{H}), 5.25(\mathrm{~s}$, 2H), 3.45-3.41 (m, 2H), $2.93(\mathrm{t}, J=7.2 \mathrm{~Hz}, 2 \mathrm{H}), 2.36(\mathrm{~s}, 3 \mathrm{H}) \mathrm{ppm} ;{ }^{13} \mathbf{C}$ NMR (101 MHz, CDCl 3$) \delta 165.6,142.1,140.2,138.6,135.8,129.0,128.6,127.7,127.6,127.1$, $127.0,126.2,124.3,116.6,49.4,27.8,23.8,21.4 \mathrm{ppm}$ (One carbon is missing because of overlapping); IR (neat) $v 3031,2922,1595,1275,1137,1091,812,760,696 \mathrm{~cm}^{-1}$. HRMS (ESI) m/z: [M + Na $]^{+}$calcd for $\mathrm{C}_{23} \mathrm{H}_{22} \mathrm{~N}_{2} \mathrm{O}_{2} \mathrm{NaS}$ 413.1294; found: 413.1297.

\section{(E)-4-methyl- $N$-(1-phenethyl-3,4-dihydroquinolin-2(1H)-}

\section{ylidene)benzenesulfonamide $(3 \mathbf{j})$}

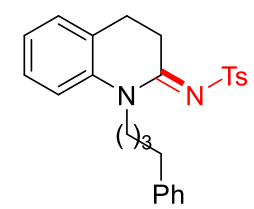

Purified by flash column chromatography (eluent: petroleum ether/ethyl acetate $=10 / 1-1 / 1$ ), white solid, $70 \mathrm{mg}, 81 \%$ yield. Mp 111$112{ }^{\circ} \mathrm{C} .{ }^{\mathbf{1}} \mathbf{H}$ NMR $\left(\mathbf{4 0 0} \mathbf{~ M H z}, \mathbf{C D C l}_{3}\right) \delta 7.83(\mathrm{~d}, J=8.4 \mathrm{~Hz}, 2 \mathrm{H}), 7.28-$ $7.22(\mathrm{~m}, 5 \mathrm{H}), 7.21-7.13(\mathrm{~m}, 2 \mathrm{H}), 7.11-7.03(\mathrm{~m}, 3 \mathrm{H}), 6.99(\mathrm{~d}, J=8.4$ $\mathrm{Hz}, 1 \mathrm{H}), 3.99(\mathrm{t}, J=7.8 \mathrm{~Hz}, 2 \mathrm{H}), 3.31-3.27(\mathrm{~m}, 2 \mathrm{H}), 2.80(\mathrm{t}, J=7.8 \mathrm{~Hz}, 2 \mathrm{H}), 2.54(\mathrm{t}, J$ $=8.0 \mathrm{~Hz}, 2 \mathrm{H}), 2.38(\mathrm{~s}, 3 \mathrm{H}), 1.75-1.68(\mathrm{~m}, 2 \mathrm{H}), 1.64-1.57(\mathrm{~m}, 2 \mathrm{H}) \mathrm{ppm} ;{ }^{\mathbf{1 3}} \mathbf{C}$ NMR $(\mathbf{1 0 1}$ MHz, CDCl3) $\delta 164.9,142.2,141.8,140.6,138.2,129.1,128.3,128.2,127.9,127.6$, $127.2,126.2,125.8,124.2,116.0,45.5,35.2,28.4,27.9,26.2,23.9,21.4$ ppm; IR (neat) v 2923, 2849, 1600, 1266, 1136, 1090, 812, $692 \mathrm{~cm}^{-1}$. HRMS (ESI) m/z: $[\mathrm{M}+\mathrm{H}]^{+}$ calcd for $\mathrm{C}_{26} \mathrm{H}_{29} \mathrm{~N}_{2} \mathrm{O}_{2} \mathrm{~S}$ 433.1944; found: 433.1936 .

\section{Methyl (E)-2-(2-(tosylimino)-3,4-dihydroquinolin-1(2H)-yl)acetate (3k)}

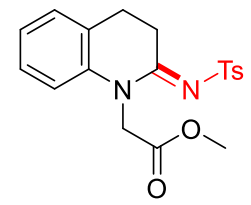

Purified by flash column chromatography (eluent: petroleum ether/ethyl acetate =10/1-1/1), white solid, $57 \mathrm{mg}$, 77\% yield. Mp 149$150{ }^{\circ} \mathrm{C} .{ }^{1} \mathbf{H}$ NMR (400 MHz, $\left.\mathbf{C D C l}_{3}\right) \delta 7.79(\mathrm{~d}, J=8.4 \mathrm{~Hz}, 2 \mathrm{H}), 7.28$ $7.25(\mathrm{~m}, 2 \mathrm{H}), 7.24-7.17(\mathrm{~m}, 2 \mathrm{H}), 7.08(\mathrm{td}, J=7.2,1.2 \mathrm{~Hz}, 1 \mathrm{H}), 6.86(\mathrm{~d}$, $J=8.0 \mathrm{~Hz}, 1 \mathrm{H}), 4.68(\mathrm{~s}, 2 \mathrm{H}), 3.66(\mathrm{~s}, 3 \mathrm{H}), 3.34-3.30(\mathrm{~m}, 2 \mathrm{H}), 2.89-2.85(\mathrm{~m}, 2 \mathrm{H}), 2.40$ (s, 3H) ppm; ${ }^{13}$ C NMR (101 MHz, CDCl 3$) \delta 168.1,166.1,142.5,140.1,138.4,129.1$, $127.8,127.8,127.0,126.3,124.6,115.6,52.4,47.6,27.6,23.7,21.4 \mathrm{ppm}$; IR (neat) $v$ 2919, 2849, 1745, 1544, 1269, 1212, 1137, 1093, 819, 765, $681 \mathrm{~cm}^{-1}$. HRMS (ESI) $\mathbf{m} / \mathbf{z}:[\mathrm{M}+\mathrm{Na}]^{+}$calcd for $\mathrm{C}_{19} \mathrm{H}_{20} \mathrm{~N}_{2} \mathrm{O}_{4} \mathrm{NaS}$ 395.1036; found: 395.1029 .

Phenyl (E)-2-(2-(tosylimino)-3,4-dihydroquinolin-1(2H)-yl)acetate (3I)<smiles>[3H]N=C1CCc2ccccc2N1CC(=O)Oc1ccccc1</smiles>

Purified by flash column chromatography (eluent: petroleum ether/ethyl acetate $=10 / 1-1 / 1)$, pale yellow oil, $61 \mathrm{mg}, 70 \%$ yield. ${ }^{1} \mathbf{H}$ NMR (400 MHz, CDCl $) \delta 7.82(\mathrm{~d}, J=8.0 \mathrm{~Hz}, 2 \mathrm{H}), 7.33-7.27(\mathrm{~m}$, $2 \mathrm{H}), 7.26-7.20(\mathrm{~m}, 5 \mathrm{H}), 7.11(\mathrm{td}, J=7.6,0.8 \mathrm{~Hz}, 1 \mathrm{H}), 7.00(\mathrm{~d}, J=8.0$ 
$\mathrm{Hz}, 1 \mathrm{H}), 6.90-6.87(\mathrm{~m}, 2 \mathrm{H}), 4.88(\mathrm{~s}, 2 \mathrm{H}), 3.37(\mathrm{t}, J=7.2 \mathrm{~Hz}, 2 \mathrm{H}), 2.90(\mathrm{t}, J=7.2 \mathrm{~Hz}$, 2H), 2.38 (s, 3H) ppm; ${ }^{13} \mathbf{C}$ NMR (101 MHz, CDCl3) $\delta$ 166.4, 166.1, 150.1, 142.6, 134.0, 138.5, 129.3, 129.2, 127.9, 127.9, 127.2, 126.4, 126.1, 124.7, 121.2, 115.7, 48.0, 27.8, 23.8, 21.4 ppm; IR (neat) $v$ 2956, 2920, 1766, 1539, 1142, 1091, 806, 754, 684 $\mathrm{cm}^{-1}$. HRMS (ESI) m/z: $\left[\mathrm{M}+\mathrm{Na}^{+}\right.$calcd for $\mathrm{C}_{24} \mathrm{H}_{22} \mathrm{~N}_{2} \mathrm{O}_{4} \mathrm{NaS}$ 457.1192; found: 457.1194.

\section{(E)- $N$-(1-((1,3-dioxan-2-yl)methyl)-3,4-dihydroquinolin-2(1H)-ylidene)-4- methylbenzenesulfonamide (3m)}

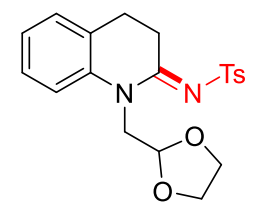

Purified by flash column chromatography (eluent: petroleum ether/ethyl acetate $=10 / 1-1 / 1)$, white solid, $60 \mathrm{mg}$, 78\% yield. Mp 159$160{ }^{\circ} \mathrm{C} .{ }^{1} \mathbf{H}$ NMR (400 MHz, CDCl3) $\delta 7.87(\mathrm{~d}, J=8.4 \mathrm{~Hz}, 2 \mathrm{H}), 7.40$ $(\mathrm{d}, J=8.0 \mathrm{~Hz}, 1 \mathrm{H}), 7.29-7.23(\mathrm{~m}, 3 \mathrm{H}), 7.16-7.14(\mathrm{~m}, 1 \mathrm{H}), 7.07(\mathrm{td}, J$ $=7.6,1.2 \mathrm{~Hz}, 1 \mathrm{H}), 5.23(\mathrm{t}, J=4.8 \mathrm{~Hz}, 1 \mathrm{H}), 4.20(\mathrm{~d}, J=4.8 \mathrm{~Hz}, 2 \mathrm{H}), 4.00-3.96(\mathrm{~m}, 2 \mathrm{H})$, 3.85-3.82 (m, 2H), 3.35-3.32 (m, 2H), $2.85(\mathrm{t}, J=7.2 \mathrm{~Hz}, 2 \mathrm{H}), 2.41(\mathrm{~s}, 3 \mathrm{H}) \mathrm{ppm} ;{ }^{13} \mathbf{C}$ NMR (101 MHz, CDCl $) \delta 165.8,142.3,140.4,139.0,129.2,127.6,127.3,126.2$, $124.4,117.3,100.964 .9,49.1,27.9,24.0,21.4 \mathrm{ppm}$ (One carbon is missing because of overlapping); IR (neat) $v$ 2953, 2892, 1593, 1407, 1271, 1134, 1091, 809, 758, $688 \mathrm{~cm}^{-}$ ${ }^{1}$. HRMS (ESI) m/z: [M + Na] ${ }^{+}$calcd for $\mathrm{C}_{20} \mathrm{H}_{22} \mathrm{~N}_{2} \mathrm{O}_{4} \mathrm{NaS}$ 409.1192; found: 409.1194 .

\section{(E)-N-(1-(2-methoxyethyl)-3,4-dihydroquinolin-2(1H)-ylidene)-4-} methylbenzenesulfonamide (3n)

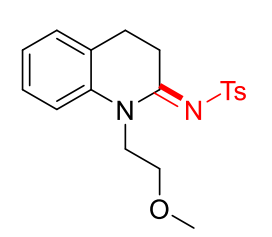

Purified by flash column chromatography (eluent: petroleum ether/ethyl acetate $=10 / 1-1 / 1)$, pale yellow oil, $52 \mathrm{mg}, 73 \%$ yield. ${ }^{1} \mathbf{H}$ NMR (400 MHz, CDCl 3$) ~ \delta 7.85(\mathrm{~d}, J=8.4 \mathrm{~Hz}, 2 \mathrm{H}), 7.35-7.33(\mathrm{~m}$, $1 \mathrm{H}), 7.30-7.23(\mathrm{~m}, 3 \mathrm{H}), 7.15(\mathrm{dd}, J=7.6,1.6 \mathrm{~Hz}, 1 \mathrm{H}), 7.07$ (td, $J=7.2$, $0.8 \mathrm{~Hz}, 1 \mathrm{H}), 4.16(\mathrm{t}, J=6.0 \mathrm{~Hz}, 2 \mathrm{H}), 3.66(\mathrm{t}, J=6.0 \mathrm{~Hz}, 2 \mathrm{H}), 3.32-$ 3.29 (m, 5H), 2.85-2.81 (m, 2H), 2.42 (s, 3H) ppm, ${ }^{13} \mathbf{C}$ NMR (101 MHz, CDCl $) \delta$ $165.4,142.3,140.5,139.0,129.2,127.7,127.6,127.2,126.2,124.4,117.2$, 69.0, 58.9, 46.5, 27.9, 24.0, 21.4 ppm; IR (neat) v 2894, 2097, 1530, 1277, 1142, 1087, 804, 753, $676 \mathrm{~cm}^{-1}$. HRMS (ESI) m/z: $[\mathrm{M}+\mathrm{H}]^{+}$calcd for $\mathrm{C}_{19} \mathrm{H}_{23} \mathrm{~N}_{2} \mathrm{O}_{3} \mathrm{~S}$ 359.1424; found: 359.1425 .

(E)-4-Methyl- $N$-(1-(2-((tetrahydro-2H-pyran-2-yl)oxy)ethyl)-3,4dihydroquinolin-2(1H)-ylidene)benzenesulfonamide (3o)

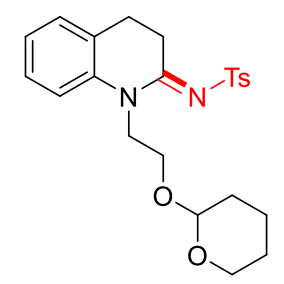

Purified by flash column chromatography (eluent: petroleum ether/ethyl acetate $=10 / 1-1 / 1)$, white solid, 56mg, 66\% yield. Mp 106$107{ }^{\circ} \mathrm{C} .{ }^{1} \mathbf{H}$ NMR $\left(400 \mathbf{~ M H z}, \mathbf{C D C l}_{3}\right) \delta 7.85(\mathrm{~d}, J=8.0 \mathrm{~Hz}, 2 \mathrm{H}), 7.39$ $(\mathrm{d}, J=8.0 \mathrm{~Hz}, 1 \mathrm{H}), 7.29-7.23(\mathrm{~m}, 3 \mathrm{H}), 7.15(\mathrm{dd}, J=7.6,1.6 \mathrm{~Hz}, 1 \mathrm{H})$, $7.07(\mathrm{td}, J=7.2,0.8 \mathrm{~Hz}, 1 \mathrm{H}), 4.52(\mathrm{t}, J=3.6 \mathrm{~Hz}, 1 \mathrm{H}), 4.29-4.16(\mathrm{~m}$, $2 \mathrm{H}), 3.99-3.93(\mathrm{~m}, 1 \mathrm{H}), 3.75-3.66(\mathrm{~m}, 2 \mathrm{H}), 3.44-3.38(\mathrm{~m}, 1 \mathrm{H}), 3.36-$ $3.25(\mathrm{~m}, 2 \mathrm{H}), 2.84(\mathrm{t}, J=7.2 \mathrm{~Hz}, 2 \mathrm{H}), 2.41(\mathrm{~s}, 3 \mathrm{H}), 1.73-1.60(\mathrm{~m}, 2 \mathrm{H}), 1.57-1.42(\mathrm{~m}$, 4H) ppm; ${ }^{13}$ C NMR (101 MHz, CDCl $) \delta$ 165.4, 142.3, 140.5, 139.0, 129.2, 127.6, 127.5, 127.3, 126.3, 124.3, 117.2, 98.8, 63.7, 62.0, 46.4, 30.4, 28.0, 25.3, 24.0, 21.4, 
19.3 ppm; IR (neat) v 2938, 2882, 1534, 1138, 1121, 1090, 1060, 1030, 972, 819, 756, $680 \mathrm{~cm}^{-1}$. HRMS (ESI) m/z: $[\mathrm{M}+\mathrm{Na}]^{+}$calcd for $\mathrm{C}_{23} \mathrm{H}_{28} \mathrm{~N}_{2} \mathrm{O}_{4} \mathrm{NaS} 451.1662$; found: 451.1662 .

\section{Ethyl (E)-4-(2-(tosylimino)-3,4-dihydroquinolin-1(2H)-yl)butanoate (3p)}

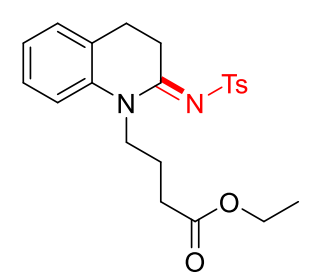

Purified by flash column chromatography (eluent: petroleum ether/ethyl acetate =10/1-1/1), white solid, $66 \mathrm{mg}, 80 \%$ yield. $\mathbf{M p}$ 93-94 ${ }^{\circ} \mathrm{C} .{ }^{1} \mathbf{H}$ NMR $\left(400 \mathrm{MHz}, \mathbf{C D C l}_{3}\right) \delta 7.85(\mathrm{~d}, J=8.0 \mathrm{~Hz}, 2 \mathrm{H})$, $7.30-7.24(\mathrm{~m}, 4 \mathrm{H}), 7.17(\mathrm{~d}, J=7.6 \mathrm{~Hz}, 1 \mathrm{H}), 7.07(\mathrm{td}, J=7.2,1.6 \mathrm{~Hz}$, $1 \mathrm{H}), 4.12(\mathrm{q}, J=7.2 \mathrm{~Hz}, 2 \mathrm{H}), 4.06-4.03(\mathrm{~m}, 2 \mathrm{H}), 3.32-3.28(\mathrm{~m}, 2 \mathrm{H})$, 2.85-2.81 (m, 2H), $2.42(\mathrm{~s}, 3 \mathrm{H}), 2.34(\mathrm{t}, J=6.8 \mathrm{~Hz}, 2 \mathrm{H}), 2.04-1.97$

(m, 2H), 1.25 (t, $J=7.2 \mathrm{~Hz}, 3 \mathrm{H}) \mathrm{ppm} ;{ }^{13} \mathbf{C}$ NMR (101 MHz, CDCl3) $\delta$ 172.8, 165.2, $142.3,140.5,138.2,129.2,127.9,127.0,126.2,124.3,116.1,60.6,45.0,31.3,27.8$, 23.9, 21.7, 21.4, $14.2 \mathrm{ppm}$ (One carbon is missing because of overlapping); IR (neat) $v$ 2938, 2849, 1535, 1275, 1140, 1091, 808, 758, 696, $682 \mathrm{~cm}^{-1}$. HRMS (ESI) m/z: [M $+\mathrm{Na}]^{+}$calcd for $\mathrm{C}_{22} \mathrm{H}_{26} \mathrm{~N}_{2} \mathrm{O}_{4} \mathrm{NaS}$ 437.1506; found: 437.1505 .

(E)-N-(1-(2-bromoethyl)-3,4-dihydroquinolin-2(1H)-ylidene)-4methylbenzenesulfonamide $(3 q)$

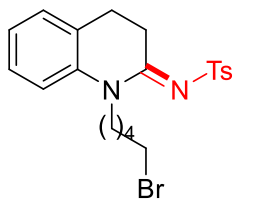

Purified by flash column chromatography (eluent: petroleum ether/ethyl acetate $=10 / 1-1 / 1$ ), pale yellow solid, $71 \mathrm{mg}, 79 \%$ yield. Mp 122-123 ${ }^{\circ} \mathrm{C} .{ }^{1} \mathbf{H}$ NMR (400 MHz, CDCl $) \delta 7.85(\mathrm{~d}, J=8.4 \mathrm{~Hz}$, $2 \mathrm{H}), 7.29(\mathrm{~d}, J=8.4 \mathrm{~Hz}, 2 \mathrm{H}), 7.27-7.24(\mathrm{~m}, 1 \mathrm{H}), 7.17(\mathrm{dd}, J=7.6,1.6$ $\mathrm{Hz}, 1 \mathrm{H}), 7.10-7.04(\mathrm{~m}, 2 \mathrm{H}), 3.98(\mathrm{t}, J=8.0 \mathrm{~Hz}, 2 \mathrm{H}), 3.32-3.28(\mathrm{~m}, 4 \mathrm{H}), 2.85-2.81(\mathrm{~m}$, 2H), 2.42 (s, 3H), 1.81-1.74 (m, 2H), 1.73-1.65 (m, 2H), 1.47-1.39 (m, 2H) ppm; ${ }^{13} \mathrm{C}$ NMR (101 MHz, CDCl 3 ) $\delta$ 164.8, 142.3, 140.5, 138.1, 129.2, 127.9, 127.7, 127.2, $126.2,124.2,115.8,45.3,33.3,31.8,27.8,25.6,25.1,23.9,21.4$ ppm; IR (neat) $v 2944$, 2866, 1545, 1273, 1140, 1085, 811, 758, $668 \mathrm{~cm}^{-1}$. HRMS (ESI) m/z: $[\mathrm{M}+\mathrm{H}]^{+}$calcd for $\mathrm{C}_{21} \mathrm{H}_{26} \mathrm{~N}_{2} \mathrm{O}_{2} \mathrm{SBr} 449.0893$; found: 449.0887 .

\section{(E)-4-Methyl- $N$-(1-phenyl-3,4-dihydroquinolin-2(1H)-}

\section{ylidene)benzenesulfonamide (3r)}

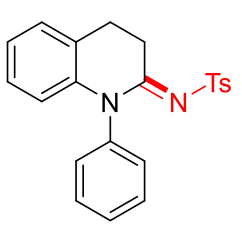

Purified by flash column chromatography (eluent: petroleum ether/ethyl acetate =10/1-1/1), white solid, $56 \mathrm{mg}$, 75\% yield. Mp 192$193{ }^{\circ} \mathrm{C} .{ }^{1} \mathbf{H}$ NMR (400 MHz, CDCl $)$ ) $\delta$ 7.51-7.46 (m, 4H), 7.44-7.39 $(\mathrm{m}, 1 \mathrm{H}), 7.22-7.18(\mathrm{~m}, 3 \mathrm{H}), 7.10(\mathrm{~d}, J=8.0 \mathrm{~Hz}, 2 \mathrm{H}), 7.05-7.03(\mathrm{~m}$, 2H), 6.39-6.37 (m, 1H), 3.55-3.51 (m, 2H), 3.06-3.02 (m, 2H), $2.33(\mathrm{~s}$, 3H) ppm, ${ }^{13} \mathbf{C}$ NMR (101 MHz, CDCl $) \delta 165.6,141.9,140.4,140.0,138.9,129.6$, $128.8,128.7,128.3,127.7,127.3,125.9,125.9,124.2,118.0,28.1,24.0,21.3$ ppm; IR (neat) $v 2917,2890,1548,1388,1271,1134,1086,807,745,681 \mathrm{~cm}^{-1}$. HRMS (ESI) $\mathbf{m} / \mathbf{z}:[\mathrm{M}+\mathrm{Na}]^{+}$calcd for $\mathrm{C}_{22} \mathrm{H}_{20} \mathrm{~N}_{2} \mathrm{O}_{2} \mathrm{NaS} 399.1138$; found: 399.1141 .

(E)- $N$-(1-(benzo[d][1,3]dioxol-5-yl)-3,4-dihydroquinolin-2(1H)-ylidene)-4methylbenzenesulfonamide (3s) 


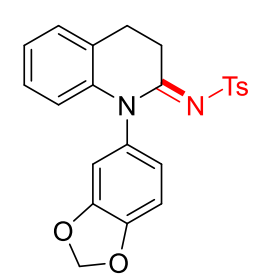

Purified by flash column chromatography (eluent: petroleum ether/ethyl acetate $=10 / 1-1 / 1)$, pale yellow solid, $66 \mathrm{mg}, 78 \%$ yield. Mp 162-163 ${ }^{\circ} \mathrm{C} .{ }^{1} \mathbf{H}$ NMR (400 MHz, CDCl 3$) \delta 7.60(\mathrm{~d}, J=8.4 \mathrm{~Hz}$, $2 \mathrm{H}), 7.20-7.15(\mathrm{~m}, 3 \mathrm{H}), 7.09-7.01(\mathrm{~m}, 2 \mathrm{H}), 6.87(\mathrm{~d}, J=8.8 \mathrm{~Hz}, 1 \mathrm{H})$, 6.65-6.63 (m, 2H), $6.48(\mathrm{dd}, J=8.0,1.6 \mathrm{~Hz}, 1 \mathrm{H}), 6.03(\mathrm{~s}, 2 \mathrm{H}), 3.48(\mathrm{t}$, $J=7.2 \mathrm{~Hz}, 2 \mathrm{H}), 3.00(\mathrm{t}, J=7.6 \mathrm{~Hz}, 2 \mathrm{H}), 2.36(\mathrm{~s}, 3 \mathrm{H}) \mathrm{ppm} ;{ }^{13} \mathbf{C} \mathbf{N M R}$ (101 MHz, CDCl3) $\delta 166.1,148.5,147.4,142.0,140.3,140.1,132.3,128.9,127.6$, 127.3, 126.0, 125.8, 124.2, 122.0, 118.0, 109.4, 108.7, 101.8, 28.0, 23.9, 21.4 ppm; IR (neat) $v 2919,2848,1536,1276,1245,833,681 \mathrm{~cm}^{-1}$. HRMS (ESI) m/z: $[\mathrm{M}+\mathrm{H}]^{+}$ calcd for $\left[\mathrm{C}_{23} \mathrm{H}_{21} \mathrm{~N}_{2} \mathrm{O}_{4} \mathrm{~S}\right]^{+} 421.1216$; found: 421.1217 .

\section{(E)-4-Methyl- $N$-(1-methylpyrrolidin-2-ylidene)benzenesulfonamide (3t)}

5 Purified by flash column chromatography (eluent: petroleum ether/ethyl I $\mathrm{N}^{-T s}$ acetate $\left.=10 / 1-1 / 1\right)$, white solid, $17 \mathrm{mg}, 33 \%$ yield. Mp $146-147{ }^{\circ} \mathrm{C} .{ }^{1} \mathbf{H}$ NMR (400 MHz, CDCl $) \delta 7.81(\mathrm{~d}, J=8.0 \mathrm{~Hz}, 2 \mathrm{H}), 7.25(\mathrm{~d}, J=8.0 \mathrm{~Hz}$, $2 \mathrm{H}), 3.45(\mathrm{t}, J=7.6 \mathrm{~Hz}, 2 \mathrm{H}), 3.03(\mathrm{t}, J=8.0 \mathrm{~Hz}, 2 \mathrm{H}), 2.96(\mathrm{~s}, 3 \mathrm{H}), 2.40(\mathrm{~s}, 3 \mathrm{H}), 2.08-$ $2.00(\mathrm{~m}, 2 \mathrm{H}) \mathrm{ppm} ;{ }^{13} \mathbf{C} \mathbf{N M R}\left(\mathbf{1 0 1} \mathbf{M H z}, \mathbf{C D C l}_{3}\right) \delta 169.8,141.9,140.5,129.0,126.4$, 51.6, 31.9, 30.6, 21.4, 18.9 ppm; IR (neat) v 2927, 1600, 1492, 1281, 1007, 902, 813, 670 $\mathrm{cm}^{-1}$. HRMS (ESI) m/z: [M + H] $]^{+}$calcd for $\mathrm{C}_{12} \mathrm{H}_{17} \mathrm{~N}_{2} \mathrm{O}_{2} \mathrm{~S}$ 253.1005; found: 253.1008.

\section{(E)-N-(1-benzylpiperidin-2-ylidene)-4-methylbenzenesulfonamide (3u)}

(6) Purified by flash column chromatography (eluent: petroleum ether/ethyl ${ }_{N} N_{N^{-T s}}$ acetate $\left.=10 / 1-1 / 1\right)$, white solid, $57 \mathrm{mg}, 83 \%$ yield. Mp $156-157{ }^{\circ} \mathrm{C} .{ }^{1} \mathbf{H}$ Bn NMR (400 MHz, CDCl $) \delta 7.77(\mathrm{~d}, J=8.0 \mathrm{~Hz}, 2 \mathrm{H}), 7.30-7.28(\mathrm{~m}, 3 \mathrm{H})$, 7.23-7.20 (m, 4H), 4.69 (s, 2H), 3.32-3.29 (m, 2H), $3.14(\mathrm{t}, J=6.0 \mathrm{~Hz} 2 \mathrm{H}), 2.38(\mathrm{~s}, 3 \mathrm{H})$, 1.78-1.75(m,4 H) ppm; ${ }^{13}$ C NMR (101 MHz, CDCl $) \delta 165.9,141.6,141.2,135.6$, 129.0, 128.6, 128.0, 127.7, 126.1, 53.8, 48.2, 28.7, 22.1, 21.3, 19.5 ppm; IR (neat) $v$ 2922, 2863, 1552, 1468, 1264, 1137, 1071, 959, 813, 699, $665 \mathrm{~cm}^{-1}$. HRMS (ESI) m/z: $[\mathrm{M}+\mathrm{H}]^{+}$calcd for $\mathrm{C}_{19} \mathrm{H}_{23} \mathrm{~N}_{2} \mathrm{O}_{2} \mathrm{~S} 343.1475$; found: 343.1480 .

\section{(E)-4-Methyl- $N$-(1-methylpiperidin-2-ylidene)benzenesulfonamide (3v)}

$\begin{array}{ll}6 & \begin{array}{l}\text { Purified by flash column chromatography (eluent: petroleum ether/ethyl } \\ \text { acetate }=10 / 1-1 / 1) \text {, white solid, } 50 \mathrm{mg}, 93 \% \text { yield. }\end{array}{ }^{\mathbf{1}} \mathbf{H} \text { NMR (400 MHz, } \\ \left.\mathbf{C D C l}_{\mathbf{N}}\right) \delta 7.80(\mathrm{~d}, J=8.0 \mathrm{~Hz}, 2 \mathrm{H}), 7.24(\mathrm{~d}, J=8.0 \mathrm{~Hz}, 2 \mathrm{H}), 3.34(\mathrm{t}, J=5.6\end{array}$ $\mathrm{Hz}, 2 \mathrm{H}), 3.02$ (s, 3H), $2.98(\mathrm{t}, J=6.4 \mathrm{~Hz}, 2 \mathrm{H}), 2.38$ (s, 3H), 1.79-1.69 (m, 4H) ppm, ${ }^{13}$ C NMR (101 MHz, CDCl3) $\delta 165.6,141.4,141.0,128.7,125.8,50.5,38.0,28.1$, $21.8,21.1,19.2 \mathrm{ppm}$. The data is consistent with that reported in the literature. ${ }^{[6]}$

\section{(E)- $N$-(1-benzylazepan-2-ylidene)-4-methylbenzenesulfonamide (3w)}

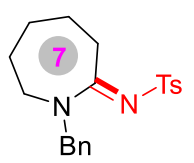

Purified by flash column chromatography (eluent: petroleum ether/ethyl acetate $=10 / 1-1 / 1)$, colorless oil , $51 \mathrm{mg}, 72 \%$ yield. ${ }^{1} \mathbf{H}$ NMR $(400 \mathrm{MHz}$, CDCl3) $\delta 7.80(\mathrm{~d}, J=8.4 \mathrm{~Hz}, 2 \mathrm{H}), 7.30-6.26(\mathrm{~m} 3 \mathrm{H}), 7.24-7.21(\mathrm{~m}, 4 \mathrm{H})$, $4.71(\mathrm{~s}, 2 \mathrm{H}), 3.44-3.42(\mathrm{~m}, 2 \mathrm{H}), 3.20(\mathrm{t}, J=6.0 \mathrm{~Hz} 2 \mathrm{H}), 2.38(\mathrm{~s}, 3 \mathrm{H}), 1.70-1.68(\mathrm{~m}, 4 \mathrm{H})$, 1.48-1.43 (m, 2H) ppm; ${ }^{13}$ C NMR (101 MHz, CDCl $) \delta 171.3,141.7,141.4,136.1$, 
129.0, 128.6, 128.3 127.7, 126.2, 53.9, 50.4, 31.4, 29.1, 26.8, 23.0, 21.4 ppm; IR (neat) v 2923, 2857, 1539, 1263, 1138, 1082, 890, 777, $682 \mathrm{~cm}^{-1}$. HRMS (ESI) m/z: $[\mathrm{M}+$ $\mathrm{Na}]^{+}$calcd for $\mathrm{C}_{20} \mathrm{H}_{24} \mathrm{~N}_{2} \mathrm{O}_{2} \mathrm{NaS} 379.1451$; found: 379.1454 .

\section{(E)- $N$-(1-benzylazocan-2-ylidene)-4-methylbenzenesulfonamide (3x)}

(8) Purified by flash column chromatography (eluent: petroleum ether/ethyl acetate $=10 / 1-1 / 1)$, white solid, $50 \mathrm{mg}$, 68\% yield. $\mathbf{M p} 96-97{ }^{\circ} \mathrm{C} .{ }^{\mathbf{1}} \mathbf{H}$ NMR (400 MHz, CDCl3) $\delta 7.74(\mathrm{~d}, J=8.4 \mathrm{~Hz}, 2 \mathrm{H}), 7.27-7.26(\mathrm{~m}, 3 \mathrm{H}), 7.21$ $7.18(\mathrm{~m}, 4 \mathrm{H}), 4.69(\mathrm{~s}, 2 \mathrm{H}), 3.50(\mathrm{t}, J=5.6 \mathrm{~Hz}, 2 \mathrm{H}), 3.14(\mathrm{t}, J=6.0 \mathrm{~Hz}, 2 \mathrm{H})$, $\mathrm{Bn}^{\prime}-\mathrm{N}^{\mathrm{N}}-\mathrm{Ts}$ $2.37(\mathrm{~s}, 3 \mathrm{H}), 2.08-2.01(\mathrm{~m}, 2 \mathrm{H}), 1.66-1.60(\mathrm{~m}, 2 \mathrm{H}), 1.58-1.47(\mathrm{~m}, 4 \mathrm{H}) \mathrm{ppm} ;{ }^{13} \mathbf{C}$ NMR
$\left(\mathbf{1 0 1} \mathbf{M H z}, \mathbf{C D C l}_{3}\right) \delta 169.8,141.5,136.1,128.9,128.5,128.1,127.6,126.1,51.1,47.4$, 29.4, 29.2, 28.7, 25.9, 24.7, 21.3 ppm; IR (neat) v 2925, 2860, 1533, 1281, 1139, 1083, 819, 728, $676 \mathrm{~cm}^{-1}$. HRMS (ESI) $\mathbf{m} / \mathbf{z}:[\mathrm{M}+\mathrm{H}]^{+}$calcd for $\mathrm{C}_{21} \mathrm{H}_{27} \mathrm{~N}_{2} \mathrm{O}_{2} \mathrm{~S} 371.1788$; found: 371.1788 .

\section{(E)- $N$-(1-benzylazonan-2-ylidene)-4-methylbenzenesulfonamide (3y)}

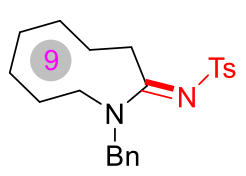

Purified by flash column chromatography (eluent: petroleum ether/ethyl acetate $=10 / 1-1 / 1)$, colorless oil, $44 \mathrm{mg}, 57 \%$ yield. ${ }^{1} \mathbf{H}$ NMR (400 MHz, CDCl3) $\delta 7.70(\mathrm{~d}, J=8.0 \mathrm{~Hz}, 2 \mathrm{H}), 7.27-7.24(\mathrm{~m}$, $3 \mathrm{H}), 7.18-7.14(\mathrm{~m}, 4 \mathrm{H}), 4.66(\mathrm{~s}, 2 \mathrm{H}), 3.51(\mathrm{t}, J=6.0 \mathrm{~Hz}, 2 \mathrm{H}), 3.12(\mathrm{~s}$, $2 \mathrm{H}), 2.36(\mathrm{~s}, 3 \mathrm{H}), 2.06-2.01(\mathrm{~m}, 2 \mathrm{H}), 1.70-1.59(\mathrm{~m}, 4 \mathrm{H}), 1.55-1.42(\mathrm{~m}, 4 \mathrm{H}) \mathrm{ppm} ;{ }^{13} \mathrm{C}$ NMR (101 MHz, CDCl 3$) \delta$ 170.3, 141.5, 141.4, 135.8, 128.9, 128.5, 128.0, 127.5, 126.0, 50.3, 48.1, 31.4, 28.4, 26.1, 25.6, 25.2, 21.3, 20.8 ppm; IR (neat) v 2924, 2863, 1529, 1452, 1261, 1138, 1084, 791, $678 \mathrm{~cm}^{-1}$. HRMS (ESI) m/z: $[\mathrm{M}+\mathrm{H}]^{+}$calcd for $\mathrm{C}_{22} \mathrm{H}_{29} \mathrm{~N}_{2} \mathrm{O}_{2} \mathrm{~S}$ 385.1944; found: 385.1946 .

\section{(E)- $N$-(1-ethyl-3-methyl-3,4-dihydroquinolin-2(1H)-ylidene)-}

\section{4methylbenzenesulfonamide $(3 \mathrm{z})^{[7,8]}$}<smiles>[3H]/N=C1\C(C)Cc2ccccc2N1[12CH3]</smiles>

Purified by flash column chromatography (eluent: petroleum ether/ethyl acetate =10/1-1/1), white solid, $21 \mathrm{mg}, 30 \%$ yield. Mp 110-

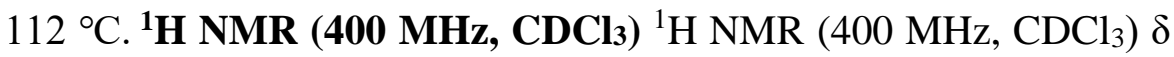
$7.88(\mathrm{~d}, J=8.4 \mathrm{~Hz}, 2 \mathrm{H}), 7.30-7.27(\mathrm{~m}, 3 \mathrm{H}), 7.18-7.16(\mathrm{~d}, J=7.2 \mathrm{~Hz}, 1 \mathrm{H}), 7.10-7.07$ $(\mathrm{m}, 2 \mathrm{H}), 4.28-4.15(\mathrm{~m}, 2 \mathrm{H}), 3.87-3.79(\mathrm{~m}, 1 \mathrm{H}), 3.12(\mathrm{dd}, J=16,5.2 \mathrm{~Hz}, 1 \mathrm{H}), 2.56(\mathrm{dd}$, $J=16,6.0 \mathrm{~Hz}, 1 \mathrm{H}), 2.42(\mathrm{~s}, 3 \mathrm{H}), 1.25(\mathrm{t}, 3 \mathrm{H}), 1.09(\mathrm{~d}, J=7.2 \mathrm{~Hz}, 3 \mathrm{H}) \mathrm{ppm} ;{ }^{13} \mathbf{C} \mathbf{~ N M R}$ (101 MHz, CDCl3) $\delta 167.8,142.1,140.9,137.4,129.14,129.13,127.6,126.2,125.1$, $124.3,115.4,40.9,31.4,31.1,21.4,15.3,12.0$ ppm; IR (neat) v 2972, 2920, 2850, 1530 , 1259, 1139, 1077, 1019, 819, 769, 755, 689, $664 \mathrm{~cm}^{-1}$.HRMS (ESI) m/z: $[\mathrm{M}+\mathrm{H}]^{+}$ calcd for $\mathrm{C}_{19} \mathrm{H}_{23} \mathrm{~N}_{2} \mathrm{O}_{2} \mathrm{~S} 343.1475$; found: 343.1476 .

(E)-N-(1-ethyl-3,4-dihydroquinolin-2(1H)-ylidene)-4methoxybenzenesulfonamide (4a) 


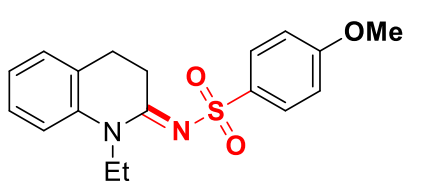

Purified by flash column chromatography (eluent: petroleum ether/ethyl acetate $=10 / 1-1 / 1)$, white solid, $45 \mathrm{mg}, 65 \%$ yield. Mp 129-130 ${ }^{\circ} \mathrm{C} .{ }^{1} \mathbf{H}$ NMR (400 MHz, CDCl $) \delta 7.91(\mathrm{~d}, J=$ $8.4 \mathrm{~Hz}, 2 \mathrm{H}), 7.29-7.25(\mathrm{~m}, 1 \mathrm{H}), 7.17(\mathrm{~d}, J=7.2 \mathrm{~Hz}, 1 \mathrm{H})$, 7.11-7.05 (m, 2H), $6.97(\mathrm{~d}, J=8.8 \mathrm{~Hz}, 2 \mathrm{H}), 4.06(\mathrm{q}, J=7.2 \mathrm{~Hz}, 2 \mathrm{H}), 3.86(\mathrm{~s}, 3 \mathrm{H}), 3.29$ (t, $J=7.2 \mathrm{~Hz}, 2 \mathrm{H}), 2.83(\mathrm{t}, J=7.2 \mathrm{~Hz}, 2 \mathrm{H}), 1.29(\mathrm{t}, J=7.2 \mathrm{~Hz}, 3 \mathrm{H}) \mathrm{ppm} ;{ }^{13} \mathbf{C} \mathbf{N M R}$ (101 MHz, CDCl3) $\delta 164.6,162.0,138.2,135.5,128.2,127.8,127.7,127.1,124.1$, 115.8, 113.7, 55.4, 41.0, 27.7, 23.9, $12.1 \mathrm{ppm}$; IR (neat) $\vee 2984,2960,1536,1256$, 1129, 1079. 1012, 815, 756, 705, $681 \mathrm{~cm}^{-1}$. HRMS (ESI) $\mathbf{m} / \mathbf{z}:[\mathrm{M}+\mathrm{H}]^{+}$calcd for $\mathrm{C}_{18} \mathrm{H}_{21} \mathrm{~N}_{2} \mathrm{O}_{3} \mathrm{~S}$ 345.1267; found: 345.1270.

(E)-N-(1-ethyl-3,4-dihydroquinolin-2(1H)-ylidene)-4(trifluoromethyl)benzenesulfonamide (4b)

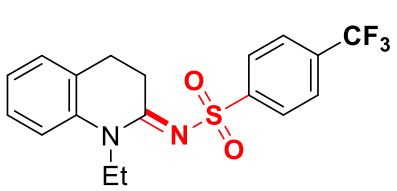

Purified by flash column chromatography (eluent: petroleum ether/ethyl acetate $=10 / 1-1 / 1)$, white solid, $73 \mathrm{mg}, 95 \%$ yield. Mp 139-140 ${ }^{\circ} \mathrm{C} .{ }^{1} \mathbf{H}$ NMR (400 MHz, CDCl $) \delta 8.12(\mathrm{~d}, J=$ $8.4 \mathrm{~Hz}, 2 \mathrm{H}), 7.77(\mathrm{~d}, J=8.0 \mathrm{~Hz}, 2 \mathrm{H}), 7.31-7.29(\mathrm{~m}, 1 \mathrm{H}), 7.21-$ $7.18(\mathrm{~m}, 1 \mathrm{H}), 7.13-7.09(\mathrm{~m}, 2 \mathrm{H}), 4.05(\mathrm{q}, J=7.2 \mathrm{~Hz}, 2 \mathrm{H}), 3.35-3.32(\mathrm{~m}, 2 \mathrm{H}), 2.89-2.85$ (m, 2H), 1.29 (t, $J=7.2 \mathrm{~Hz}, 3 \mathrm{H}) \mathrm{ppm} ;{ }^{13} \mathbf{C}$ NMR (151 MHz, CDCl $) \delta 165.1,146.8$, 138.0, $133.4\left(\mathrm{q},{ }^{2} J_{\mathrm{C}-\mathrm{F}}=32.9 \mathrm{~Hz}\right), 128.0,127.8,127.1,126.8,125.8\left(\mathrm{q},{ }^{3} J_{\mathrm{C}-\mathrm{F}}=3.8 \mathrm{~Hz}\right)$, 124.6, 123.4(q, $\left.{ }^{1} J_{\mathrm{C}-\mathrm{F}}=273.2 \mathrm{~Hz}\right), 116.0,41.3,28.3,23.8,12.1 \mathrm{ppm} ;{ }^{19} \mathbf{F}$ NMR (376 MHz, CDCl 3 ) $\delta-62.99$ ppm; IR (neat) v 2988, 2954, 1609, 1324, 1269, 1121, 1061, 819, 758, 718, $641 \mathrm{~cm}^{-1}$. HRMS (ESI) $\mathbf{m} / \mathbf{z}:[\mathrm{M}+\mathrm{H}]^{+}$calcd for $\mathrm{C}_{18} \mathrm{H}_{18} \mathrm{~N}_{2} \mathrm{O}_{2} \mathrm{~F}_{3} \mathrm{~S}$ 383.1036; found: 383.1027 .

\section{(E)-4-(Tert-butyl)- $\mathrm{N}$-(1-ethyl-3,4-dihydroquinolin-2(1H)-} ylidene)benzenesulfonamide (4c)

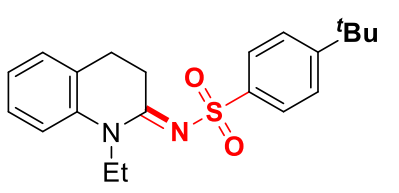

Purified by flash column chromatography (eluent: petroleum ether/ethyl acetate $=10 / 1-1 / 1)$, white solid, $66 \mathrm{mg}, 89 \%$ yield. Mp 102-103 ${ }^{\circ} \mathrm{C} .{ }^{1} \mathbf{H}$ NMR (400 MHz, CDCl3) $\delta 7.90(\mathrm{~d}, J=$ $8.4 \mathrm{~Hz}, 2 \mathrm{H}), 7.49(\mathrm{~d}, J=8.4 \mathrm{~Hz}, 2 \mathrm{H}), 7.29-7.24(\mathrm{~m}, 1 \mathrm{H}), 7.17$ $(\mathrm{dd}, J=7.2,1.2 \mathrm{~Hz}, 1 \mathrm{H}), 7.11-7.05(\mathrm{~m}, 2 \mathrm{H}), 4.08(\mathrm{q}, J=7.2 \mathrm{~Hz}, 2 \mathrm{H}), 3.32-3.28(\mathrm{~m}$, 2H), 2.84-2.81(m, 2H), 1.34 (s, 9H), 1.31 (t, $J=7.2 \mathrm{~Hz}, 3 \mathrm{H}) \mathrm{ppm} ;{ }^{13} \mathbf{C}$ NMR (101 MHz, $\left.\mathbf{C D C l}_{3}\right) \delta 164.8,155.2,140.4,138.2,127.8,127.6,127.1,126.0,125.5,124.2,115.8$, 41.0, 34.9, 31.0, 27.7, 23.8, 12.1 ppm; IR (neat) v 2957, 2906, 1540, 1274, 1146, 1080, 757, $649 \mathrm{~cm}^{-1}$.HRMS (ESI) $\mathbf{m} / \mathbf{z}:[\mathrm{M}+\mathrm{H}]^{+}$calcd for $\mathrm{C}_{21} \mathrm{H}_{27} \mathrm{~N}_{2} \mathrm{O}_{2} \mathrm{~S} 371.1788$; found: 371.1780 .

\section{(E)-4-Chloro- $N$-(1-ethyl-3,4-dihydroquinolin-2(1H)-ylidene)benzenesulfonamide} (4d)

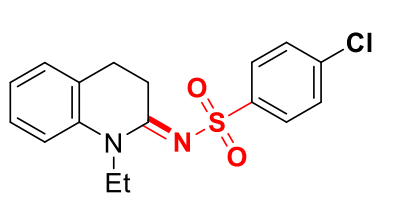

Purified by flash column chromatography (eluent: petroleum ether/ethyl acetate $=10 / 1-1 / 1$ ), white solid, $50 \mathrm{mg}, 72 \%$ yield. Mp 164-165 ${ }^{\circ} \mathrm{C} .{ }^{1} \mathbf{H}$ NMR (400 MHz, CDCl3) $\delta 7.92(\mathrm{~d}, J=$ $8.8 \mathrm{~Hz}, 2 \mathrm{H}), 7.46$ (d, $J=8.8 \mathrm{~Hz}, 2 \mathrm{H}), 7.30-7.25$ (m, 1H), 7.19- 
$7.17(\mathrm{~m}, 1 \mathrm{H}), 7.12-7.07(\mathrm{~m}, 2 \mathrm{H}), 4.05(\mathrm{q}, J=7.2 \mathrm{~Hz}, 2 \mathrm{H}), 3.30(\mathrm{t}, J=7.2 \mathrm{~Hz} 2 \mathrm{H}), 2.84$ $(\mathrm{t}, J=7.2 \mathrm{~Hz}, 2 \mathrm{H}), 1.28(\mathrm{t}, J=7.2 \mathrm{~Hz}, 3 \mathrm{H}) \mathrm{ppm} ;{ }^{13} \mathbf{C ~ N M R}(\mathbf{1 0 1} \mathbf{M H z}, \mathbf{C D C l} 3) \delta 164.8$, 142.0, 138.0, 137.9, 128.8, 127.9, 127.8, 127.7, 127.1, 124.4, 115.8, 41.2, 28.0, 23.8, $12.1 \mathrm{ppm}$; IR (neat) $v 2983,2956,1533,1281,1080,818,758,649 \mathrm{~cm}^{-1}$. HRMS (ESI) $\mathbf{m} / \mathbf{z}:\left([\mathrm{M}+\mathrm{Na}]^{+}\right.$calcd for $\mathrm{C}_{17} \mathrm{H}_{17} \mathrm{~N}_{2} \mathrm{O}_{2} \mathrm{NaSCl} 371.0591$; found: 371.0586 .

\section{(E)-3,5-Dichloro- $N$-(1-ethyl-3,4-dihydroquinolin-2(1H)-} ylidene)benzenesulfonamide (4e)

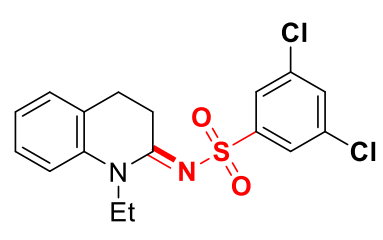

Purified by flash column chromatography (eluent: petroleum ether/ethyl acetate $=10 / 1-1 / 1)$, white solid, $64 \mathrm{mg}, 84 \%$ yield. Mp 210-21 ${ }^{\circ} \mathrm{C} .{ }^{1} \mathbf{H}$ NMR (400 MHz, CDCl $) \delta 7.86(\mathrm{~s}, 2 \mathrm{H})$, $7.50(\mathrm{~s}, 1 \mathrm{H}), 7.31-7.27(\mathrm{~m}, 1 \mathrm{H}), 7.20(\mathrm{~d}, J=7.2 \mathrm{~Hz}, 1 \mathrm{H}), 7.16-$ $7.09(\mathrm{~m}, 2 \mathrm{H}), 4.06(\mathrm{q}, J=7.2 \mathrm{~Hz}, 2 \mathrm{H}), 3.31(\mathrm{t}, J=7.2 \mathrm{~Hz}, 2 \mathrm{H})$, $2.87(\mathrm{t}, J=7.2 \mathrm{~Hz}, 2 \mathrm{H}), 1.30(\mathrm{t}, J=7.2 \mathrm{~Hz}, 3 \mathrm{H}) \mathrm{ppm} ;{ }^{\mathbf{1 3}} \mathbf{C} \mathbf{N M R}\left(\mathbf{1 0 1} \mathbf{M H z}, \mathbf{C D C l}_{3}\right) \delta$ 165.1, 146.1, 137.9, 135.4, 131.6, 128.0, 127.8, 127.1, 124.8, 124.7, 116.0, 41.4, 28.3, 23.8, $12.1 \mathrm{ppm}$; IR (neat) v 3074, 2979, 1528, 1378, 1274, 1155, 1121, 1086, 1010, 755, 670, $638 \mathrm{~cm}^{-1}$. HRMS (ESI) $\mathbf{m} / \mathbf{z}:[\mathrm{M}+\mathrm{Na}]^{+}$calcd for $\mathrm{C}_{17} \mathrm{H}_{16} \mathrm{~N}_{2} \mathrm{O}_{2} \mathrm{NaSCl}_{2}$ 405.0202; found: 405.0188 .

(E)- $N$-(1-ethyl-3,4-dihydroquinolin-2(1H)-ylidene)naphthalene-1-sulfonamide (4f)

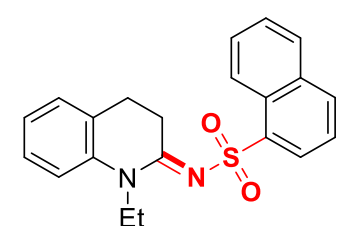
Purified by flash column chromatography (eluent: petroleum ether/ethyl acetate $=10 / 1-1 / 1)$, white solid, $61 \mathrm{mg}, 84 \%$ yield. Mp 210-21 ${ }^{\circ} \mathrm{C} .{ }^{1} \mathbf{H}$ NMR (400 MHz, CDCl $) \delta 8.84(\mathrm{~d}, J=8.4 \mathrm{~Hz}$, $1 \mathrm{H}), 8.35(\mathrm{~d}, J=7.2 \mathrm{~Hz}, 1 \mathrm{H}), 8.02(\mathrm{~d}, J=8.4 \mathrm{~Hz}, 1 \mathrm{H}), 7.91(\mathrm{~d}, J$ $=8.4 \mathrm{~Hz}, 1 \mathrm{H}), 7.63(\mathrm{t}, J=7.6 \mathrm{~Hz}, 1 \mathrm{H}), 7.55(\mathrm{q}, J=8.0 \mathrm{~Hz}, 2 \mathrm{H})$,

7.27-7.22 (m, 1H), $7.15(\mathrm{~d}, J=7.2 \mathrm{~Hz}, 1 \mathrm{H}), 7.07-7.04(\mathrm{~m}, 2 \mathrm{H}), 4.00(\mathrm{q}, J=7.2 \mathrm{~Hz}, 2 \mathrm{H})$, $3.32(\mathrm{t}, J=7.2 \mathrm{~Hz}, 2 \mathrm{H}), 2.82(\mathrm{t}, J=7.2 \mathrm{~Hz}, 2 \mathrm{H}), 1.20(\mathrm{t}, J=7.2 \mathrm{~Hz}, 3 \mathrm{H}) \mathrm{ppm} ;{ }^{13} \mathbf{C}$ NMR (101 MHz, CDCl $) \delta 164.9,138.5,138.2,134.2,133.3,128.53,128.50,127.9$, $127.7,127.4,127.1,126.9,126.5,126.2,124.3,124.0,115.9,41.1,27.8,23.9,12.4$ ppm; IR (neat) v 2979, 2922, 1528, 1044, 1275, 1155, 1120, 1063, 975, 835, 798, 755, 669, $638 \mathrm{~cm}^{-1}$. HRMS (ESI) m/z: $[\mathrm{M}+\mathrm{Na}]^{+}$calcd for $\mathrm{C}_{21} \mathrm{H}_{20} \mathrm{~N}_{2} \mathrm{O}_{2} \mathrm{NaS} 387.1138$; found: 387.1135 .

(E)-N-(1-ethyl-3,4-dihydroquinolin-2(1H)-ylidene)-2,4,6triisopropylbenzenesulfonamide $(4 \mathrm{~g})$<smiles>CCN1C(=NS(=O)(=O)c2c(C(C)C)cc(C(C)C)cc2C(F)(F)F)CCc2ccccc21</smiles>
Purified by flash column chromatography (eluent: petroleum ether/ethyl acetate =10/1-1/1), white solid, $48 \mathrm{mg}, 55 \%$ yield. Mp 109-1 $10{ }^{\circ} \mathrm{C} .{ }^{\mathbf{1}} \mathbf{H}$ NMR (400 $\left.\mathbf{~ M H z , ~} \mathbf{C D C l}_{3}\right) \delta$ 7.28-7.24 $(\mathrm{m}, 1 \mathrm{H}), 7.16-7.14(\mathrm{~m}, 3 \mathrm{H}), 7.11-7.03(\mathrm{~m}, 2 \mathrm{H}), 4.42-4.35(\mathrm{~m}$ $2 \mathrm{H}), 4.08(\mathrm{q}, J=7.2 \mathrm{~Hz}, 2 \mathrm{H}), 3.22-3.19(\mathrm{~m}, 2 \mathrm{H}), 2.92-2.86(\mathrm{~m}, 1 \mathrm{H}), 2.79-2.76(\mathrm{~m}, 2 \mathrm{H})$, 1.29-1.25 (m, 21H) ppm; ${ }^{13}$ C NMR (101 MHz, CDCl3) $\delta 164.3,151.6,149.1,138.3$, $136.5,127.8,127.7,127.2,123.9,123.2,115.8,40.5,34.1,29.5,27.9,24.8,24.0,23.6$, $12.2 \mathrm{ppm}$; IR (neat) v 2962, 2930, 1527, 1277, 1253, 1122, 809, 752, 673, $647 \mathrm{~cm}^{-1}$. HRMS (ESI) m/z: $[\mathrm{M}+\mathrm{H}]^{+}$calcd for $\mathrm{C}_{26} \mathrm{H}_{37} \mathrm{~N}_{2} \mathrm{O}_{2} \mathrm{~S} 441.2570$; found: 441.2567 . 
1-((1R,4R)-7,7-dimethyl-2-oxobicyclo[2.2.1]heptan-1-yl)-N-((E)-1-ethyl-3,4dihydroquinolin-2(1H)-ylidene)methanesulfonamide (4h)

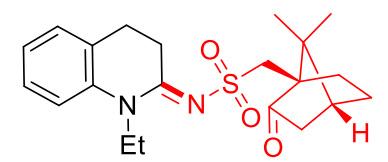

Purified by flash column chromatography (eluent: petroleum ether/ethyl acetate $=10 / 1-1 / 1)$, colorless oil, $40 \mathrm{mg}, 52 \%$ yield. ${ }^{1}$ H NMR (400 MHz, CDCl 3 ) $\delta$ 7.29-7.25 (m, 1H), 7.17 (d, $J=$ $7.2 \mathrm{~Hz}, 1 \mathrm{H}), 7.11(\mathrm{~d}, J=8.4 \mathrm{~Hz}, 1 \mathrm{H}), 7.07(\mathrm{t}, J=7.2 \mathrm{~Hz}, 1 \mathrm{H})$, 4.10-4.04(m, 2H), $3.76(\mathrm{~d}, J=15.6 \mathrm{~Hz}, 1 \mathrm{H}), 3.28(\mathrm{t}, J=7.2 \mathrm{~Hz}, 2 \mathrm{H}), 3.09$ (d, $J=15.2$ $\mathrm{Hz}, 1 \mathrm{H}), 2.87-2.82(\mathrm{~m}, 2 \mathrm{H}), 2.76-2.69(\mathrm{~m}, 1 \mathrm{H}), 2.38$ (dt, $J=18.4,4.4 \mathrm{~Hz}, 1 \mathrm{H}), 2.12-$ $2.03(\mathrm{~m}, 2 \mathrm{H}), 1.94(\mathrm{~d}, J=18.4 \mathrm{~Hz}, 1 \mathrm{H}), 1.77-1.70(\mathrm{~m} \mathrm{1H}), 1.46-1.40(\mathrm{~m}, 1 \mathrm{H}), 1.31(\mathrm{t}$, $J=7.2 \mathrm{~Hz}, 3 \mathrm{H}), 1.16(\mathrm{~s}, 3 \mathrm{H}), 0.91(\mathrm{~s}, 3 \mathrm{H}) \mathrm{ppm} ;{ }^{13} \mathbf{C}$ NMR (101 MHz, CDCl$) \delta ~ 215.2$, 164.7, 138.2, 127.8, 127.6, 127.2, 124.0, 115.8, 58.3, 51.3, 47.9, 42.6, 42.5, 40.6, 28.0, 26.9, 24.4, 23.8, 19.9, 19.7, 12.2 ppm; IR (neat) v 2958, 1741, 1536, 1461, 1411, 1293, 1274, 1118, 818, 751, 722, 636, 620, $490 \mathrm{~cm}^{-1}$. HRMS (ESI) m/z: $[\mathrm{M}+\mathrm{Na}]^{+}$calcd for $\mathrm{C}_{21} \mathrm{H}_{28} \mathrm{~N}_{2} \mathrm{O}_{3} \mathrm{NaS}$ 411.1713; found: 411.1714.

(E)- $\mathrm{N}$-(6-(4-(1-cyclohexyl-1H-tetrazol-5-yl)butoxy)-1-ethyl-3,4-dihydroquinolin2(1H)-ylidene)-4-methylbenzenesulfonamide (6a)

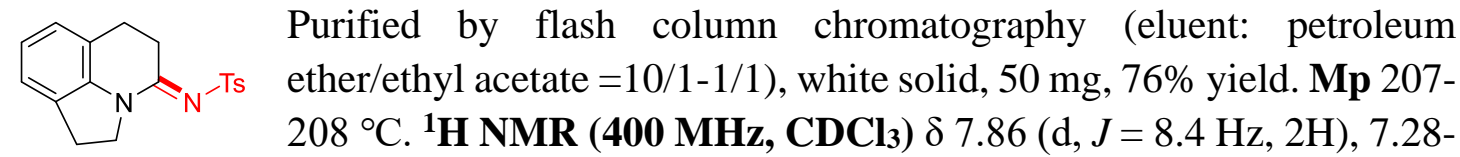
$7.26(\mathrm{~m}, 2 \mathrm{H}), 7.08(\mathrm{t}, J=4.4 \mathrm{~Hz}, 1 \mathrm{H}), 6.99-6.98(\mathrm{~m}, 2 \mathrm{H}), 4.09(\mathrm{t}, J=8.0 \mathrm{~Hz}, 2 \mathrm{H}), 3.34$ $(\mathrm{t}, J=8.0 \mathrm{~Hz}, 2 \mathrm{H}), 3.17(\mathrm{t}, J=8.4 \mathrm{~Hz}, 2 \mathrm{H}), 2.92(\mathrm{t}, J=8.0 \mathrm{~Hz}, 2 \mathrm{H}), 2.40(\mathrm{~s}, 3 \mathrm{H}) \mathrm{ppm}$; ${ }^{13}$ C NMR (101 MHz, CDCl3) $\delta 161.1,142.2,140.5,139.1,129.9,129.1,126.4,125.4$, 124.9, 123.5, 120.6, 47.7, 27.4, 27.2, 22.6, 21.4 ppm; IR (neat) $\vee 2920,2850,1519$, 1484, 1284, 1146, 1085, 807, 761, 676, 657, 597, 552, $534 \mathrm{~cm}^{-1}$. HRMS (ESI) m/z: [M $+\mathrm{Na}]^{+}$calcd for $\mathrm{C}_{18} \mathrm{H}_{18} \mathrm{~N}_{2} \mathrm{O}_{2} \mathrm{NaS} 349.0981$; found: 349.0975 .

(E)-N-(6-(4-(4-(2,3-dichlorophenyl)piperazin-1-yl)butoxy)-1-ethyl-3,4dihydroquinolin-2(1H)-ylidene)-4-methylbenzenesulfonamide (6b)

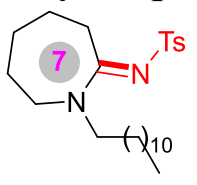

Purified by flash column chromatography (eluent: petroleum ether/ethyl acetate =10/1-1/1), colorless oil, $61 \mathrm{mg}, 70 \%$ yield. ${ }^{1}$ H NMR (400 MHz, $\left.\mathrm{CDCl}_{3}\right) \delta 7.80(\mathrm{~d}, J=8.0 \mathrm{~Hz}, 2 \mathrm{H}), 7.23(\mathrm{~d}, J=8.0 \mathrm{~Hz}, 2 \mathrm{H}), 3.49-3.44(\mathrm{~m}$, $4 \mathrm{H}), 3.12-3.09(\mathrm{~m}, 2 \mathrm{H}), 2.39(\mathrm{~s}, 3 \mathrm{H}), 1.73-1.61(\mathrm{~m}, 6 \mathrm{H}), 1.54-1.50(\mathrm{~m}$, 2H), 1.31-1.22 (m, 18H), 0.88 (t, $J=6.8 \mathrm{~Hz}, 3 \mathrm{H}) \mathrm{ppm} ;{ }^{\mathbf{1 3}} \mathbf{C}$ NMR (101 MHz, CDCl3) $\delta 170.6,141.7,141.5,128.9,126.1,51.5,51.2,31.9,31.5,29.6,29.5,29.4,29.3,29.27$, $29.24,27.4,27.1,26.8,23.0,22.6,21.4,14.1 \mathrm{ppm}$ (One carbon is missing because of overlapping); IR (neat) v 2922, 2852, 1546, 1489, 1466, 1434, 1266, 1141, 1085, 892, 844, 775, $686 \mathrm{~cm}^{-1}$. HRMS (ESI) $\mathbf{m} / \mathbf{z}:[\mathrm{M}+\mathrm{H}]^{+}$calcd for $\mathrm{C}_{25} \mathrm{H}_{43} \mathrm{~N}_{2} \mathrm{O}_{2} \mathrm{~S}$ 435.3040; found: 435.3041 .

(E)-4-Methyl- $N$-(1,2,5,6-tetrahydro-4H-pyrrolo[3,2,1-ij]quinolin-4ylidene)benzenesulfonamide (6c) 


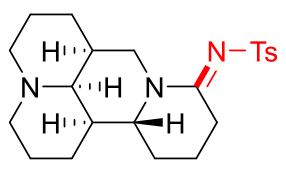

Purified by flash column chromatography (eluent: petroleum ether/ethyl acetate $=10 / 1-1 / 1)$, white solid, $65 \mathrm{mg}, 81 \%$ yield. Mp $153-154{ }^{\circ} \mathrm{C},[\alpha]_{\mathrm{D}}{ }^{25}=+63.4\left(c=0.50, \mathrm{CH}_{3} \mathrm{Cl}\right) .{ }^{1} \mathbf{H}$ NMR $(400 \mathrm{MHz}$, $\left.\mathbf{C D C l}_{3}\right) \delta 7.81(\mathrm{~d}, J=8.0 \mathrm{~Hz}, 2 \mathrm{H}), 7.24(\mathrm{~d}, J=8.0 \mathrm{~Hz}, 2 \mathrm{H}), 4.57$ (dd, $J=12.8,4.0 \mathrm{~Hz}, 1 \mathrm{H}), 3.93-3.89(\mathrm{~m}, 1 \mathrm{H}), 3.28-3.16(\mathrm{~m}, 2 \mathrm{H}), 2.84-2.70(\mathrm{~m}, 3 \mathrm{H})$, 2.39 (s, 3H), 2.04-1.70 (m, 8H), 1.68-1.38(m, 9H) ppm; ${ }^{13} \mathbf{C}$ NMR (101 MHz, CDCl $)$ $\delta 165.5,141.5,141.5,129.0,126.1,63.1,57.0,56.9,54.2,45.2,43.3,35.3,29.2,27.6$, 26.2, 25.9, 21.4, 21.0, 20.6, 17.2 ppm; IR (neat) v 2921, 2858, 1546, 1478, 1398, 1287, 1133, 1081, 973, 946, 815, 694, $674 \mathrm{~cm}^{-1}$. HRMS (ESI) m/z: $[\mathrm{M}+\mathrm{H}]^{+}$calcd for $\mathrm{C}_{22} \mathrm{H}_{32} \mathrm{~N}_{3} \mathrm{O}_{2} \mathrm{~S}$ 402.2210; found: 402.2205 .

\section{(E)-4-Methyl- $N$-(1-propylazepan-2-ylidene)benzenesulfonamide (6d)}

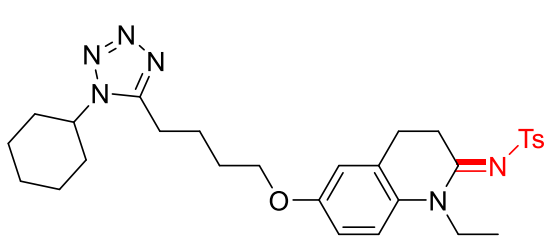

Purified by flash column chromatography (eluent: petroleum ether/ethyl acetate $=10 / 1-1 / 1)$, white solid, $72 \mathrm{mg}, 65 \%$ yield. Mp $132-133^{\circ} \mathrm{C} .{ }^{1} \mathbf{H}$ NMR (400 MHz, CDCl 3$) \delta 7.84(\mathrm{~d}, J=8.0 \mathrm{~Hz}, 2 \mathrm{H}), 7.30-$ $7.27(\mathrm{~m}, 2 \mathrm{H}), 7.01(\mathrm{~d}, J=8.8 \mathrm{~Hz}, 1 \mathrm{H}), 6.76(\mathrm{dd}, J=$ 8.8, $2.8 \mathrm{~Hz}, 1 \mathrm{H}), 6.71(\mathrm{~s}, 1 \mathrm{H}), 4.19-4.11(\mathrm{~m}, 1 \mathrm{H}), 4.06-3.98(\mathrm{~m}, 4 \mathrm{H}), 3.24(\mathrm{t}, J=7.2$ $\mathrm{Hz}, 2 \mathrm{H}), 2.93(\mathrm{t}, J=7.6 \mathrm{~Hz}, 2 \mathrm{H}), 2.77(\mathrm{t}, J=7.2 \mathrm{~Hz}, 2 \mathrm{H}), 2.41(\mathrm{~s}, 3 \mathrm{H}), 2.05-1.89(\mathrm{~m}$, 12H), 1.44-1.33 (m, 2H), 1.27 (t, $J=7.2 \mathrm{~Hz}, 3 \mathrm{H}) \mathrm{ppm} ;{ }^{\mathbf{1 3}} \mathbf{C}$ NMR (101 MHz, CDCl $)$ $\delta 164.0,155.3,153.4,142.1,140.6,131.5,129.1,128.7,126.1,116.9,114.0,112.9$, 67.3, 57.4, 41.1, 32.7, 28.3, 27.6, 25.1, 24.7, 24.1, 23.8, 22.8, 21.4, $12.1 \mathrm{ppm}$; IR (neat) v 2934, 2861, 1534, 1502, 1442, 1258, 1130, 1079, 864, 812, 760, 704, $663 \mathrm{~cm}^{-1}$. HRMS (ESI) m/z: $[\mathrm{M}+\mathrm{H}]^{+}$calcd for $\mathrm{C}_{29} \mathrm{H}_{39} \mathrm{~N}_{6} \mathrm{O}_{3} \mathrm{~S}$ 551.2799; found: 551.2793.

(4 $\left.{ }^{1} \mathrm{~S}, 7 \mathrm{aS}, 13 \mathrm{aR}, 13 \mathrm{bR}, \mathrm{E}\right)-10$-(tosylmethylene)dodecahydro-1 $H, 5 H, 8 H$ dipyrido[2,1-f:3',2',1'-ij] $][1,6]$ naphthyridine (6e)

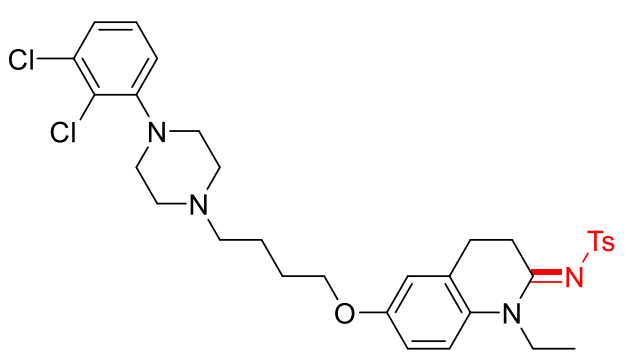

Purified by flash column chromatography (eluent: petroleum ether/ethyl acetate $=10 / 1$ 1/1), white solid, $107 \mathrm{mg}, 85 \%$ yield. Mp 136$137{ }^{\circ} \mathrm{C} .{ }^{\mathbf{1}} \mathbf{H}$ NMR (400 MHz, CDCl $) \delta 7.86(\mathrm{~d}$, $J=8.0 \mathrm{~Hz}, 2 \mathrm{H}), 7.29-7.27(\mathrm{~m}, 2 \mathrm{H}), 7.15-7.13$ $(\mathrm{m}, 2 \mathrm{H}), 7.05(\mathrm{~d}, J=8.0 \mathrm{~Hz}, 1 \mathrm{H}), 6.95(\mathrm{dd}, J=$ $6.4,3.6 \mathrm{~Hz}, 1 \mathrm{H}), 6.66(\mathrm{~d}, J=2.4 \mathrm{~Hz}, 1 \mathrm{H}), 6.59$ $(\mathrm{dd}, J=8.4,2.0 \mathrm{~Hz}, 1 \mathrm{H}), 4.05-3.97(\mathrm{~m}, 4 \mathrm{H}), 3.28-3.25(\mathrm{~m}, 2 \mathrm{H}), 3.06(\mathrm{~s}, 4 \mathrm{H}), 2.75(\mathrm{t}, J$ $=7.6 \mathrm{~Hz}, 2 \mathrm{H}), 2.65(\mathrm{~s}, 4 \mathrm{H}), 2.49(\mathrm{t}, J=7.6 \mathrm{~Hz}, 2 \mathrm{H}), 2.41(\mathrm{~s}, 3 \mathrm{H}), 1.87-1.80(\mathrm{~m}, 2 \mathrm{H})$, 1.76-1.68 (m, 2H) $1.28(\mathrm{t}, J=7.2 \mathrm{~Hz}, 3 \mathrm{H}) \mathrm{ppm} ;{ }^{13} \mathbf{C}$ NMR (101 MHz, CDCl 3$) \delta 164.9$, 158.6, 151.1, 142.2, 140.5, 139.0, 133.9, 129.1, 128.3, 127.4, 127.3, 126.2, 124.5, 119.1, $118.5,108.4$, 104.1, 67.9, 58.1, 53.2, 51.2, 41.1, 28.2, 27.2, 23.3, 23.1, 21.4, 12.1 ppm; IR (neat) v 2943, 2872, 1537, 1513, 1442, 1271, 1140, 1097, 899, 684, $674 \mathrm{~cm}^{-1}$. HRMS (ESI) $\mathbf{m} / \mathbf{z}:[\mathrm{M}+\mathrm{H}]^{+}$calcd for $\mathrm{C}_{32} \mathrm{H}_{39} \mathrm{~N}_{4} \mathrm{O}_{3} \mathrm{SCl}_{2}$ 629.2114; found: 629.2109 .

\subsection{Gram-scale synthesis}



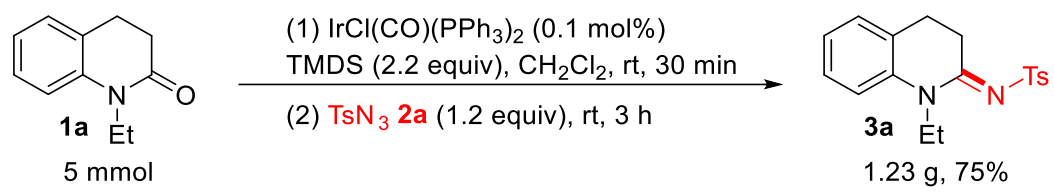

To a solution of $\mathrm{IrCl}(\mathrm{CO})\left(\mathrm{PPh}_{3}\right)_{2}$ in $\mathrm{CH}_{2} \mathrm{Cl}_{2}(25 \mathrm{~mL} ; 0.005 \mathrm{mmol}$ of [ $\left.\mathrm{Ir}]\right)$ was added the lactam 1a $(5 \mathrm{mmol})$ and TMDS $(1.47 \mathrm{~g}, 11 \mathrm{mmol})$. The solution was stirred for $30 \mathrm{~min}$ at RT. The sulfone azide $\mathbf{2 a}(6 \mathrm{mmol})$ was added and stirred at RT for $3 \mathrm{~h}$. The solvent was removed under reduced pressure and the residue was purified by flash chromatography on silica gel using a mixture of ethyl acetate/petroleum ether as eluent to yield amidine $\mathbf{3 a}$ (1.23 $\mathrm{g}, 75 \%$ yield).

\section{Preliminary mechanistic studies}

NMR experiments were performed to identify the intermediates present at each stage of the reaction. Pure starting material is shown in spectrum A, Figure S1 and starting material with TMDS is shown in spectrum B. On addition of Vaska's complex, clean conversion to the hemiaminal after 30 mins was witnessed in spectrum $\mathrm{C}$.

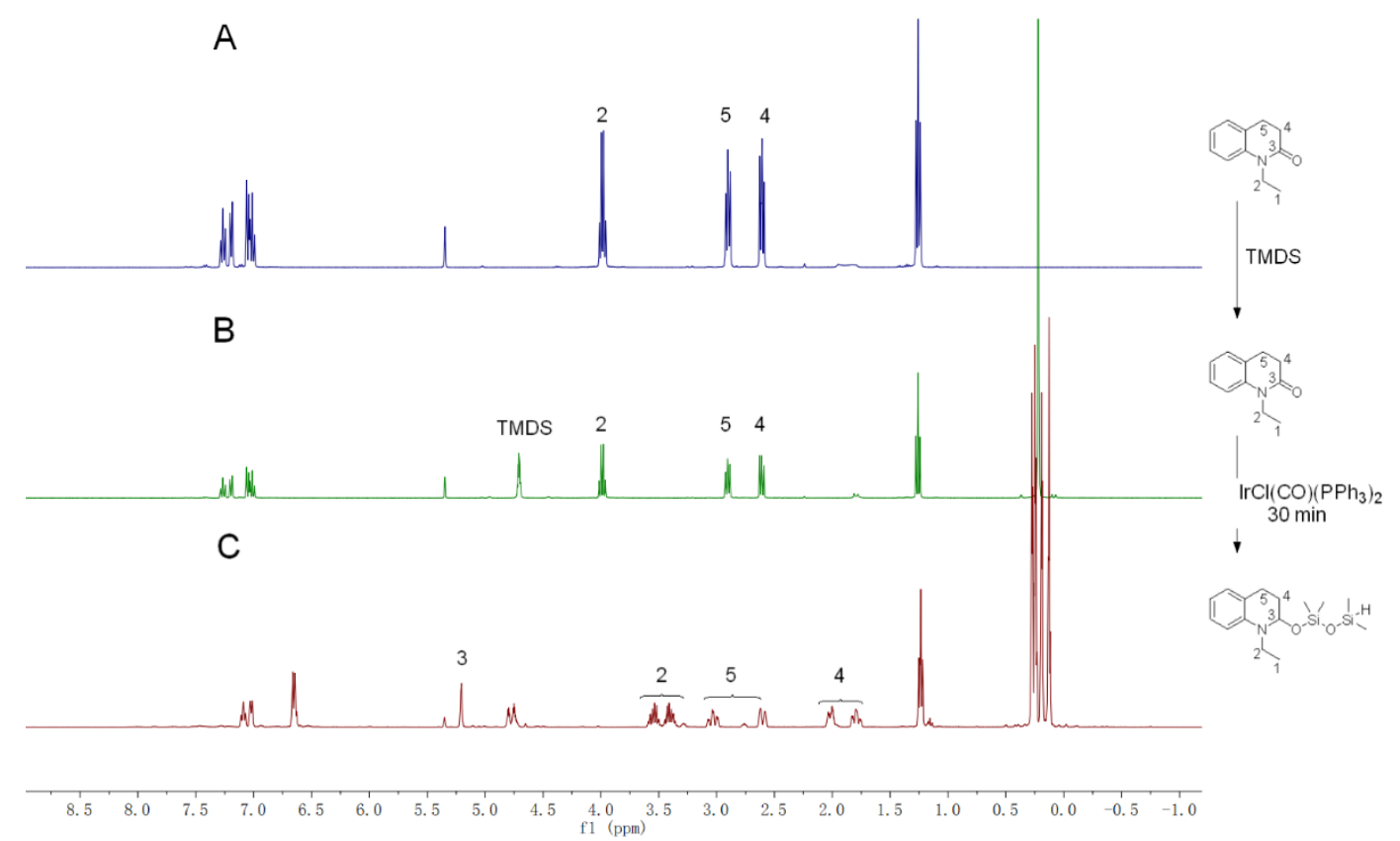

Figure S1. ${ }^{1} \mathrm{H}$ NMR $\left(400 \mathrm{M}, \mathrm{CD}_{2} \mathrm{Cl}_{2}\right)$ spectra of starting material and intermediate. 

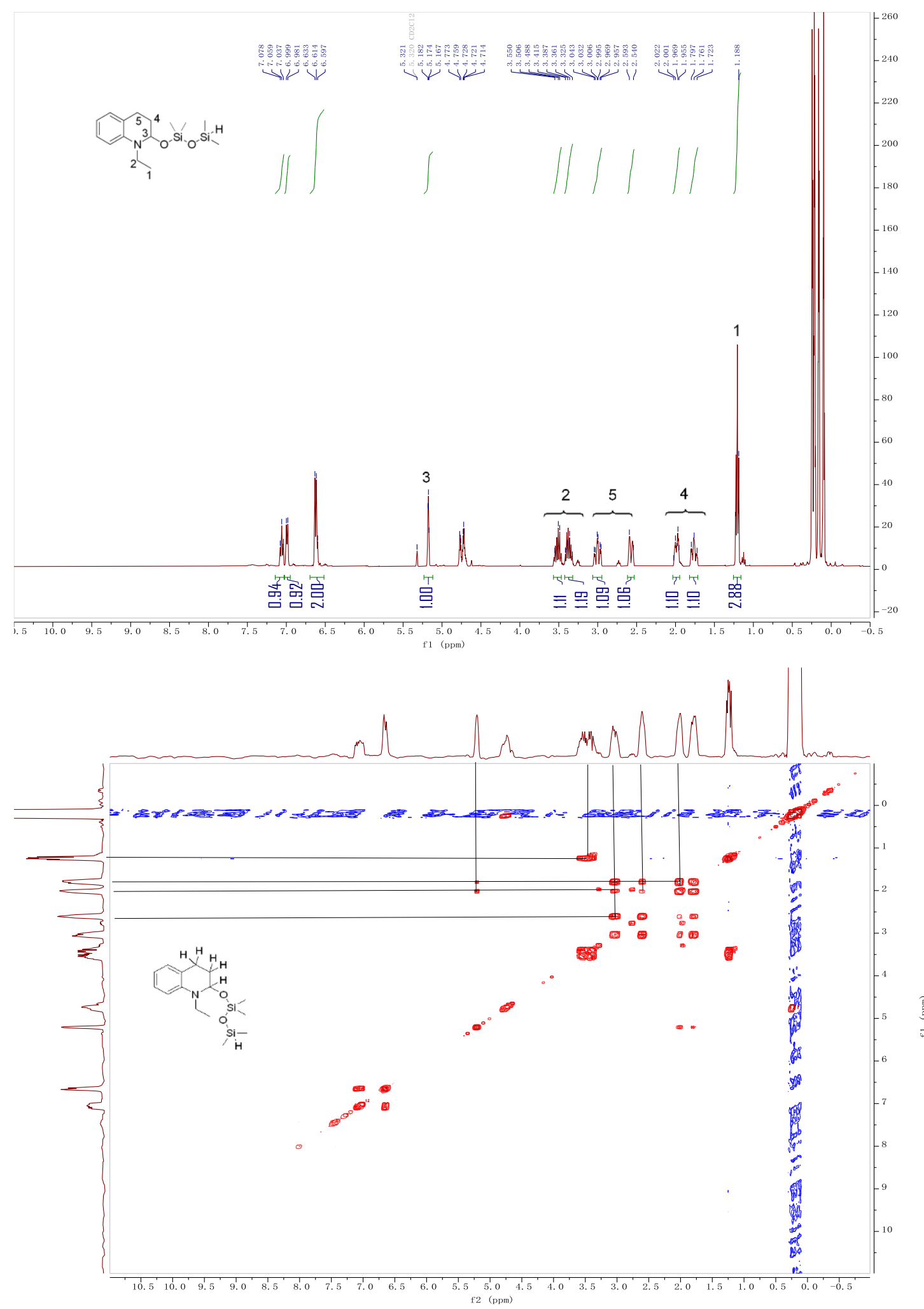

Figure S2. ${ }^{1} \mathrm{H}$ NMR $\left(400 \mathrm{M}, \mathrm{CD}_{2} \mathrm{Cl}_{2}\right)$ and ${ }^{1} \mathrm{H},{ }^{1} \mathrm{H}-\mathrm{COSY}\left(400 \mathrm{M}, \mathrm{CD}_{2} \mathrm{Cl}_{2}\right)$ spectra of the hemiaminal intermediate.

NMR experiments were performed to identify whether sulfonyl azides were compatible with the Vaska's complex. TsN 3 and Ir complex were stirred in $\mathrm{CD}_{2} \mathrm{Cl}_{2}$ at $\mathrm{rt}$ for $18 \mathrm{~h}$ and no decomposition product was observed (spectrum A, Figure S3). In addition, no 
aziridine was detected when styrene was treated with $\mathrm{TsN}_{3}$ and Vaska's complex in $\mathrm{CD}_{2} \mathrm{Cl}_{2}$ at $\mathrm{rt}$ for $18 \mathrm{~h}$ (spectrum C).

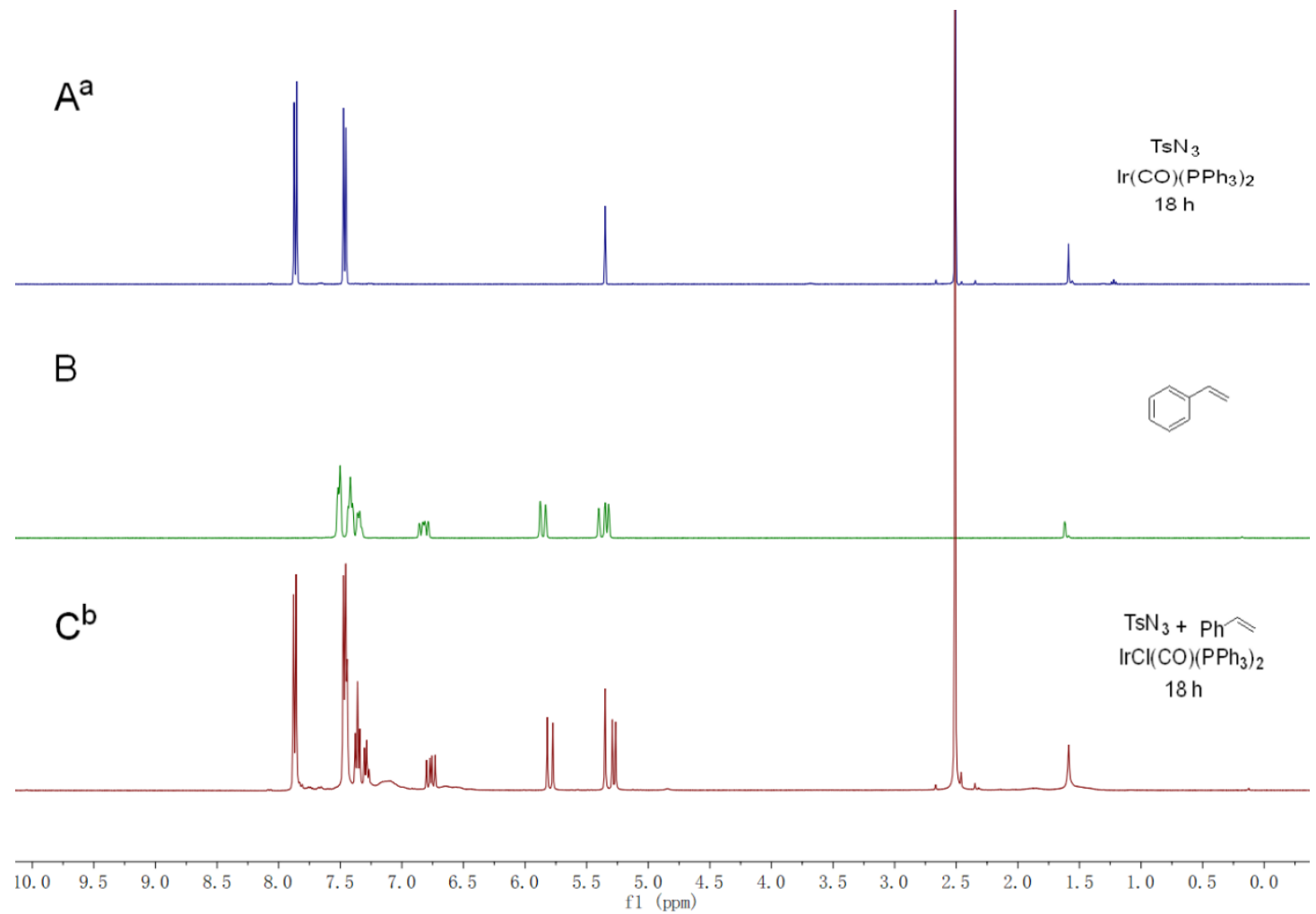

Figure S3. The investigation of compatibility of sulfonyl azides with the Vaska's complex by ${ }^{1} \mathrm{H}$ NMR $\left(400 \mathrm{M}, \mathrm{CD}_{2} \mathrm{Cl}_{2}\right)$. ${ }^{a}$ Reaction condition: $\mathrm{TsN}_{3}(0.1 \mathrm{mmol})$, $\operatorname{IrCl}(\mathrm{CO})\left(\mathrm{PPh}_{3}\right)_{2}(1.0 \mathrm{~mol} \%)$ in $\mathrm{CD}_{2} \mathrm{Cl}_{2}(0.5 \mathrm{~mL})$ were stirred at $\mathrm{rt}$ under Ar atmosphere for $18 \mathrm{~h}$. ${ }^{b}$ Reaction condition: $\mathrm{TsN}_{3}(0.1 \mathrm{mmol})$, styrene $(0.1 \mathrm{mmol})$ and $\mathrm{IrCl}(\mathrm{CO})\left(\mathrm{PPh}_{3}\right)_{2}(1.0 \mathrm{~mol} \%)$ in $\mathrm{CD}_{2} \mathrm{Cl}_{2}(0.5 \mathrm{~mL})$ were stirred at $\mathrm{rt}$ under Ar atmosphere for $18 \mathrm{~h}$.

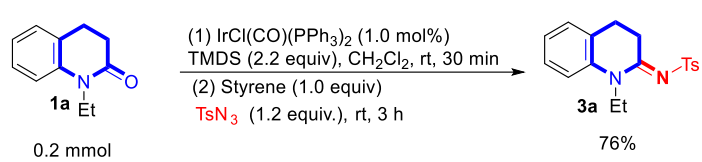

Styrene ( 1 equiv) was added into the standard reaction together with the addition of $\mathrm{TsN}_{3}$ to capture the possible iridium-nitrene intermediate. However, the reaction still gave the product $3 \mathbf{a}$ in $76 \%$ yield and no aziridine was observed.

\section{Crystallographic data}

Single crystals suitable for X-ray diffraction were obtained by slow evaporation of the solvent from $\mathrm{CH}_{2} \mathrm{Cl}_{2}$ and petroleum ether at rt. X-ray structure of $3 \mathbf{a}$ with $50 \%$ ellipsoid probability. Crystal data have been deposited to CCDC, number 2021109. 


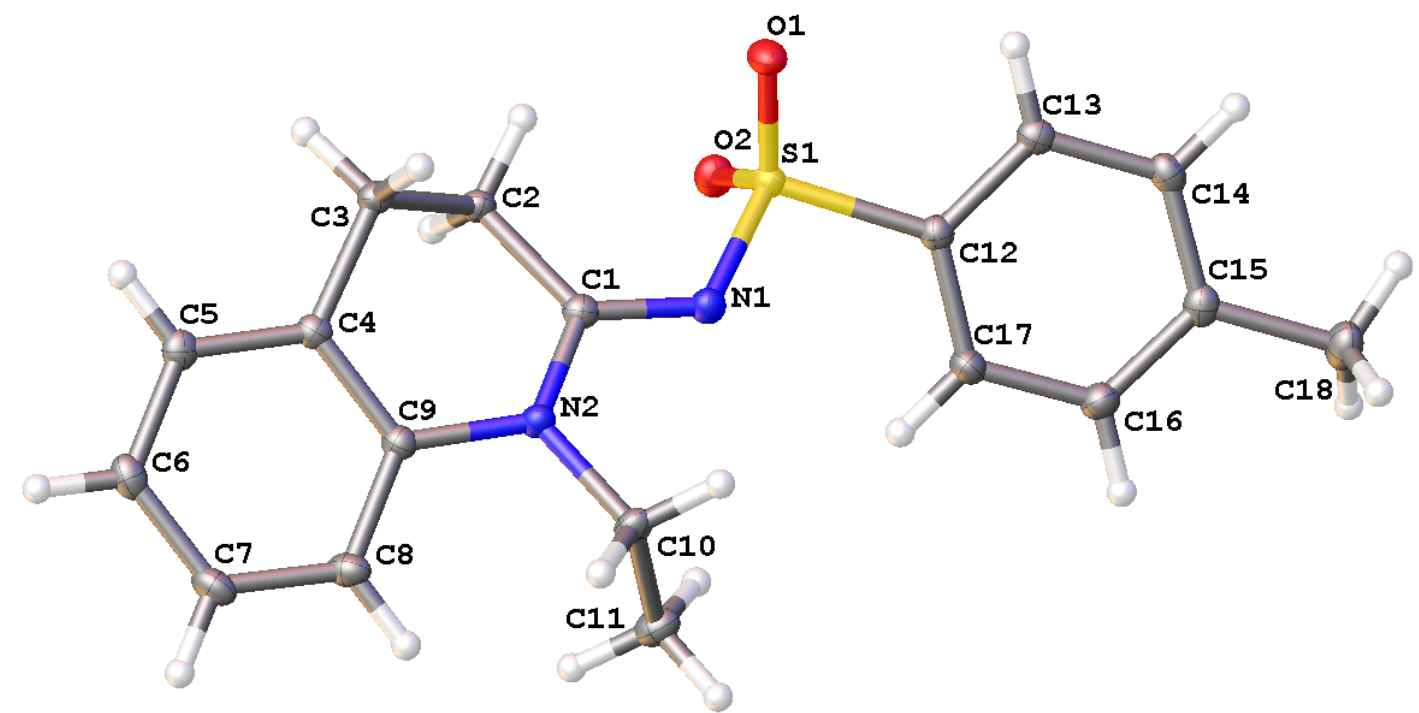

Figure S4. X-Ray crystal structure of 3a.

Table 1. Crystal data and structure refinement for $\mathbf{3 a}$.

Identification code

Empirical formula

Formula weight

Temperature

Wavelength

Crystal system

Space group

Unit cell dimensions

Volume

Z

Density (calculated)

Absorption coefficient

$\mathrm{F}(000)$

Crystal size

Theta range for data collection

Index ranges

Reflections collected

Independent reflections 3a

$\mathrm{C}_{18} \mathrm{H}_{20} \mathrm{~N}_{2} \mathrm{O}_{2} \mathrm{~S}$

328.42

$173.01 \mathrm{~K}$

$1.34139 \AA$

Monoclinic

P 1 21/c 1

$a=17.7841(15) \AA \quad \alpha=90^{\circ}$.

$\mathrm{b}=6.2339(5) \AA \quad \beta=106.775(3)^{\circ}$.

$\mathrm{c}=15.0312(13) \AA \quad \gamma=90^{\circ}$.

1595.5(2) $\AA^{3}$

4

$1.367 \mathrm{Mg} / \mathrm{m}^{3}$

$1.229 \mathrm{~mm}^{-1}$

696

$0.12 \times 0.08 \times 0.08 \mathrm{~mm}^{3}$

6.579 to $54.999^{\circ}$.

$-21<=\mathrm{h}<=21,-7<=\mathrm{k}<=7,-17<=\mathrm{l}<=18$

13776

$2989[\mathrm{R}($ int $)=0.0564]$ 
Completeness to theta $=53.594^{\circ}$

Absorption correction

Max. and min. transmission

Refinement method

Data / restraints / parameters

Goodness-of-fit on $\mathrm{F}^{2}$

Final R indices [I $>2 \operatorname{sigma}(\mathrm{I})]$

$\mathrm{R}$ indices (all data)

Extinction coefficient

Largest diff. peak and hole
$98.3 \%$

Semi-empirical from equivalents

0.7508 and 0.5643

Full-matrix least-squares on $\mathrm{F}^{2}$

2989 / 0 / 210

1.093

$\mathrm{R} 1=0.0515, \mathrm{wR} 2=0.1419$

$\mathrm{R} 1=0.0526, \mathrm{wR} 2=0.1433$

$\mathrm{n} / \mathrm{a}$

0.400 and -0.589 e. $\AA^{-3}$ 


\section{Reference}

[1] Gui, J.; Xie, H.; Jiang, H.; Zeng, W. Visible-Light-Mediated Sulfonylimination of Tertiary Amines with Sulfonylazides Involving $\mathrm{Csp}^{3}-\mathrm{Csp}^{3}$ Bond Cleavage. Org. Lett. 2019, 21, 2804-2807.

[2] Lan, Y.; Chen, Y.; Xu, X.; Qiu, Y.; Liu, S.; Liu, X.; Liu, B. F.; Zhang, G. Synthesis and biological evaluation of a novel sigma-1 receptor antagonist based on 3,4-dihydro-2(1H)-quinolinone scaffold as a potential analgesic. Eur. J. Med. Chem. 2014, 79, 216-230.

[3] Kuang, Z.; Li, B.; Song, Q. Cu/Pd cooperatively catalyzed tandem intramolecular anti-Markovnikov hydroarylation of unsaturated amides: facile construction of 3,4-dihydroquinolinones via borylation/intramolecular $\mathrm{C}\left(\mathrm{sp}^{3}\right)-\mathrm{C}\left(\mathrm{sp}^{2}\right)$ cross coupling. Chem. Commun. 2018, 54, 34-37.

[4] Durak, L. J.; Lewis, J. C. Iridium-Promoted, Palladium-Catalyzed Direct Arylation of Unactivated Arenes. Organometallics 2014, 33, 620-623.

[5] Ruiz Espelt, L.; Wiensch, E. M.; Yoon, T. P. Brønsted Acid Cocatalysts in Photocatalytic Radical Addition of $\alpha$ Amino C-H Bonds across Michael Acceptors. J. Org. Chem. 2013, 78, 4107-4114.

[6] Zhang, L.; Su, J. H.; Wang, S.; Wan, C.; Zha, Z.; Du, J.; Wang, Z. Direct electrochemical imidation of aliphatic aminesvia anodic oxidation. Chem. Commun. 2011, 47, 5488-5490.

[7] Bailey, A. S.; Baldry, P. A.; Scott, P. W. Examination of the Reactions of Hexahydropyrrolo [3,2,1 -jk]carbazole and of Pyrrolo[3,2,1 -hi] indoles with Arenesulphonyl Azides in Non-polar and Polar Solvents. J. Chem. Soc., Perkin Trans. 1, 1979, 2387-2392.

[8] Critchley, S. R.; Prout, K.; Watkin, D.; Bailey, A. S.; Peach, J. M. Crystal and molecular structure of cis5,6,6a,7,8,9,10,10a-octahydro-5-methyl-10a- $p$-tolylsulphonylamino-6-p-tolylsulphonyliminophenanthridine. $J$. Chem. Soc., Perkin Trans. 2, 1977, 1335-1338. 


\section{NMR Spectra}

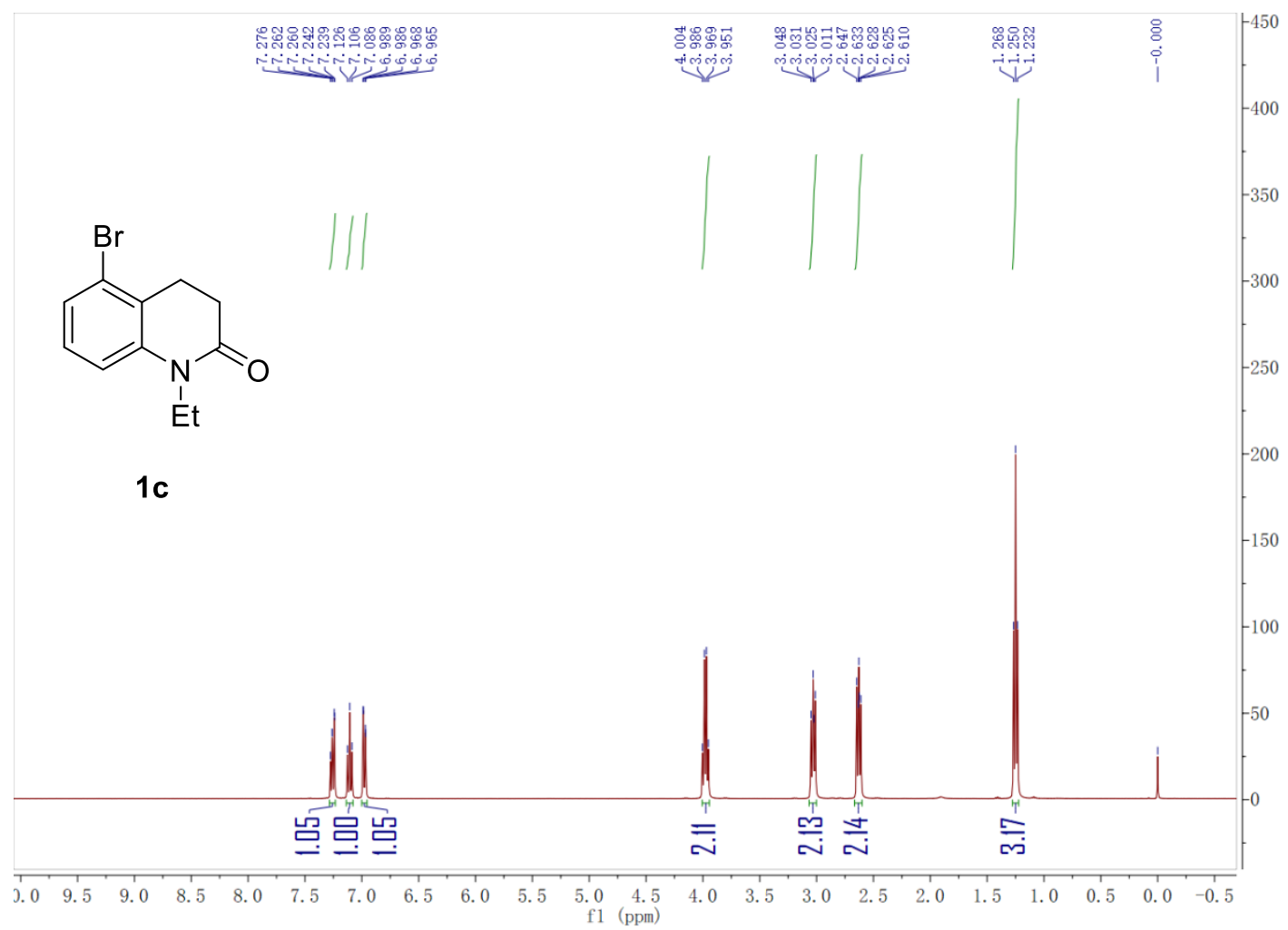

${ }^{1} \mathrm{H}$ NMR spectra $\left(400 \mathrm{MHz}, \mathrm{CDCl}_{3}\right)$ of $\mathbf{1 c}$

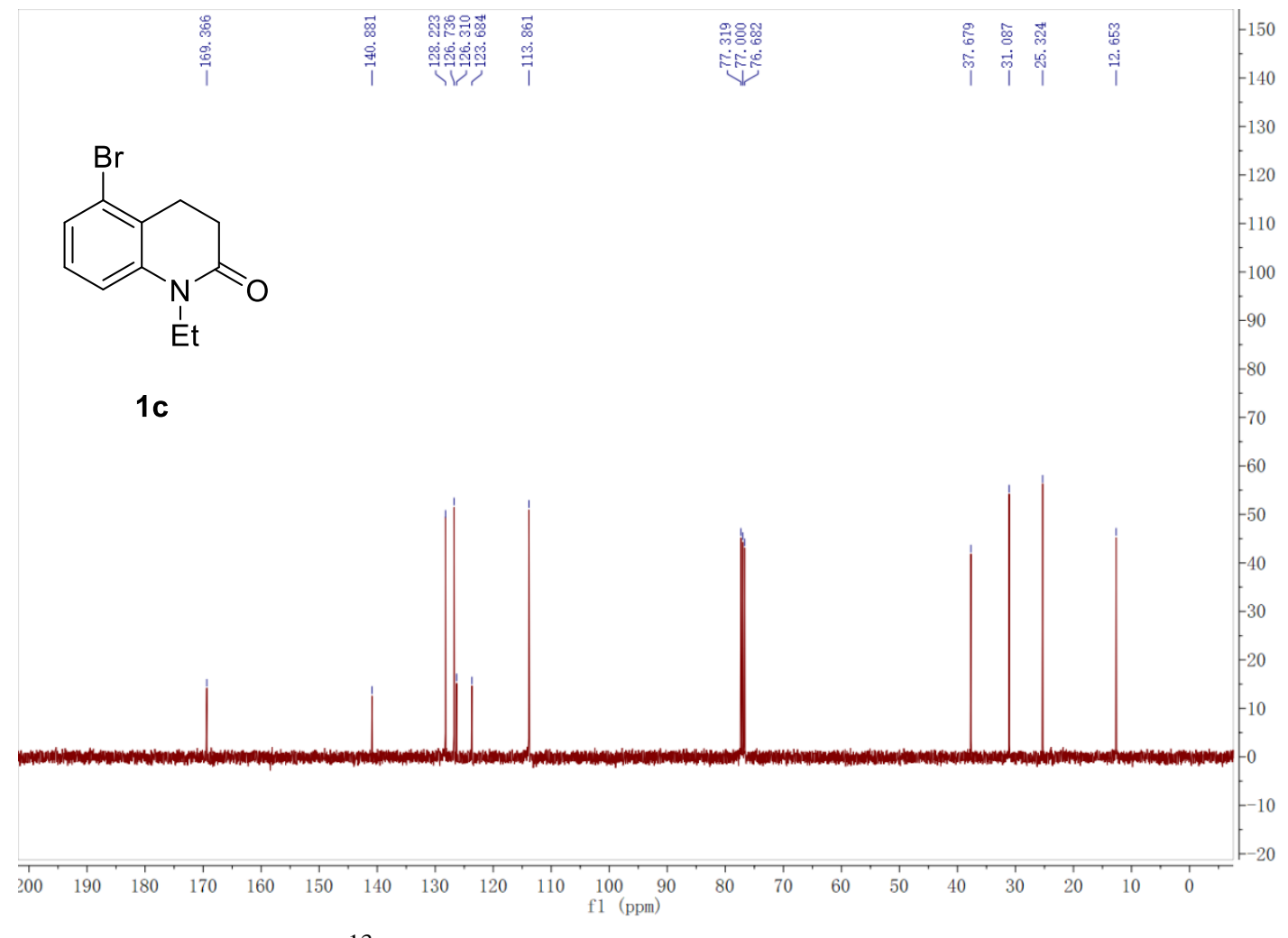

${ }^{13} \mathrm{C}$ NMR spectra $\left(101 \mathrm{MHz}, \mathrm{CDCl}_{3}\right)$ of $\mathbf{1 c}$ 


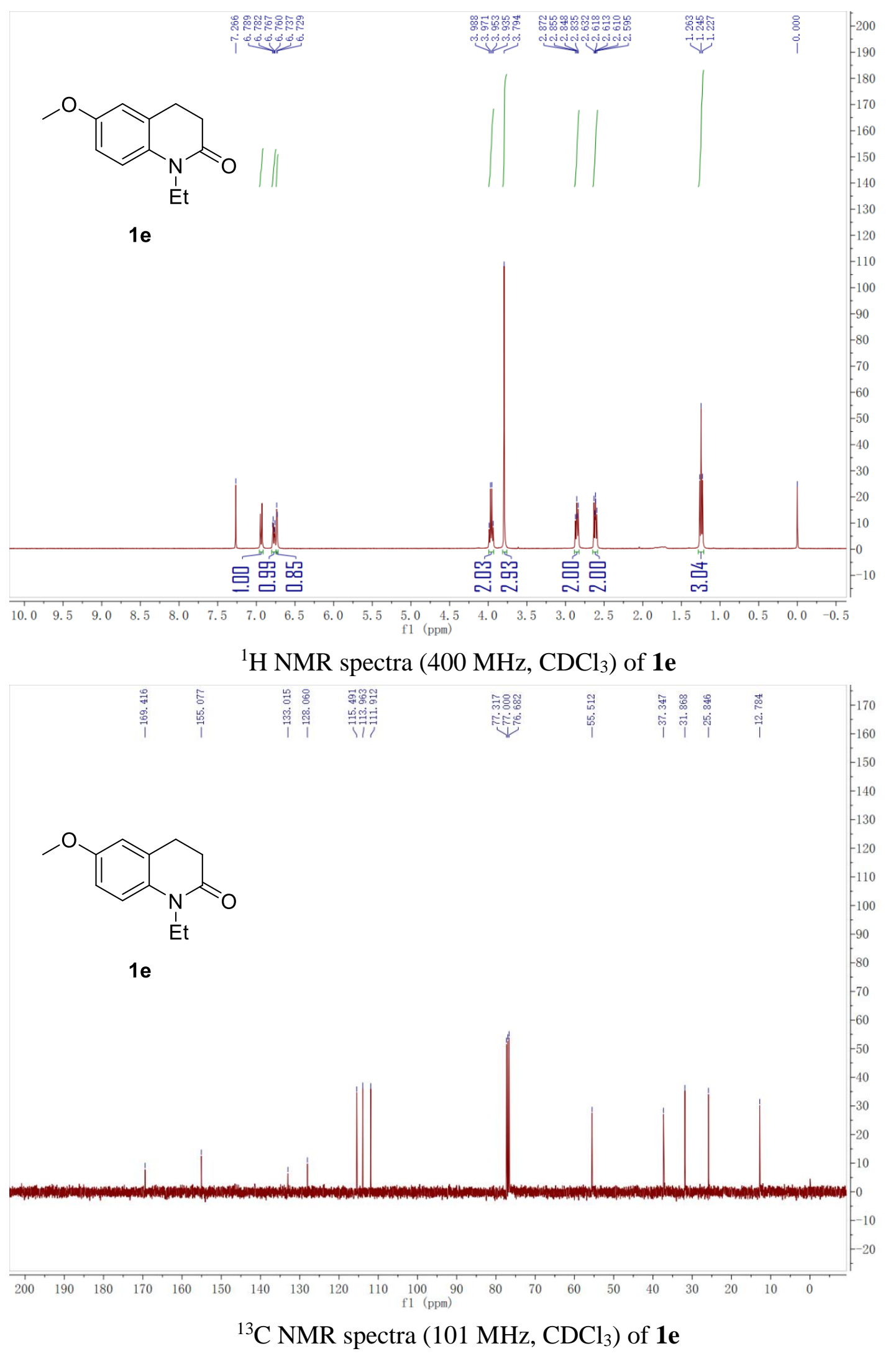



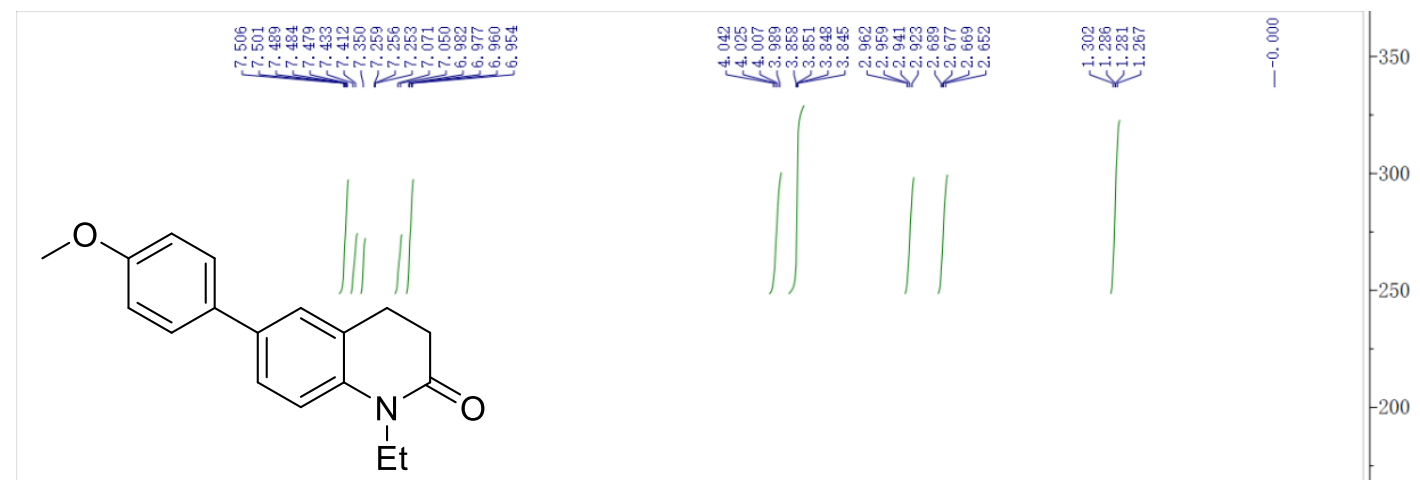

$1 f$

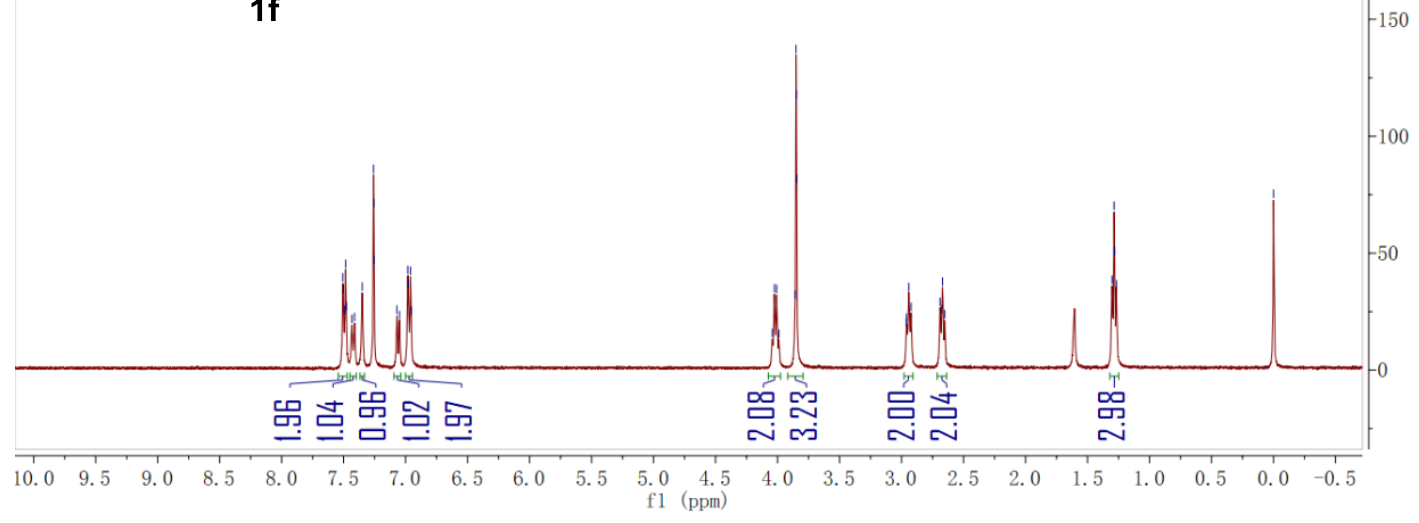

${ }^{1} \mathrm{H}$ NMR spectra (400 MHz, $\mathrm{CDCl}_{3}$ ) of $\mathbf{1 f}$

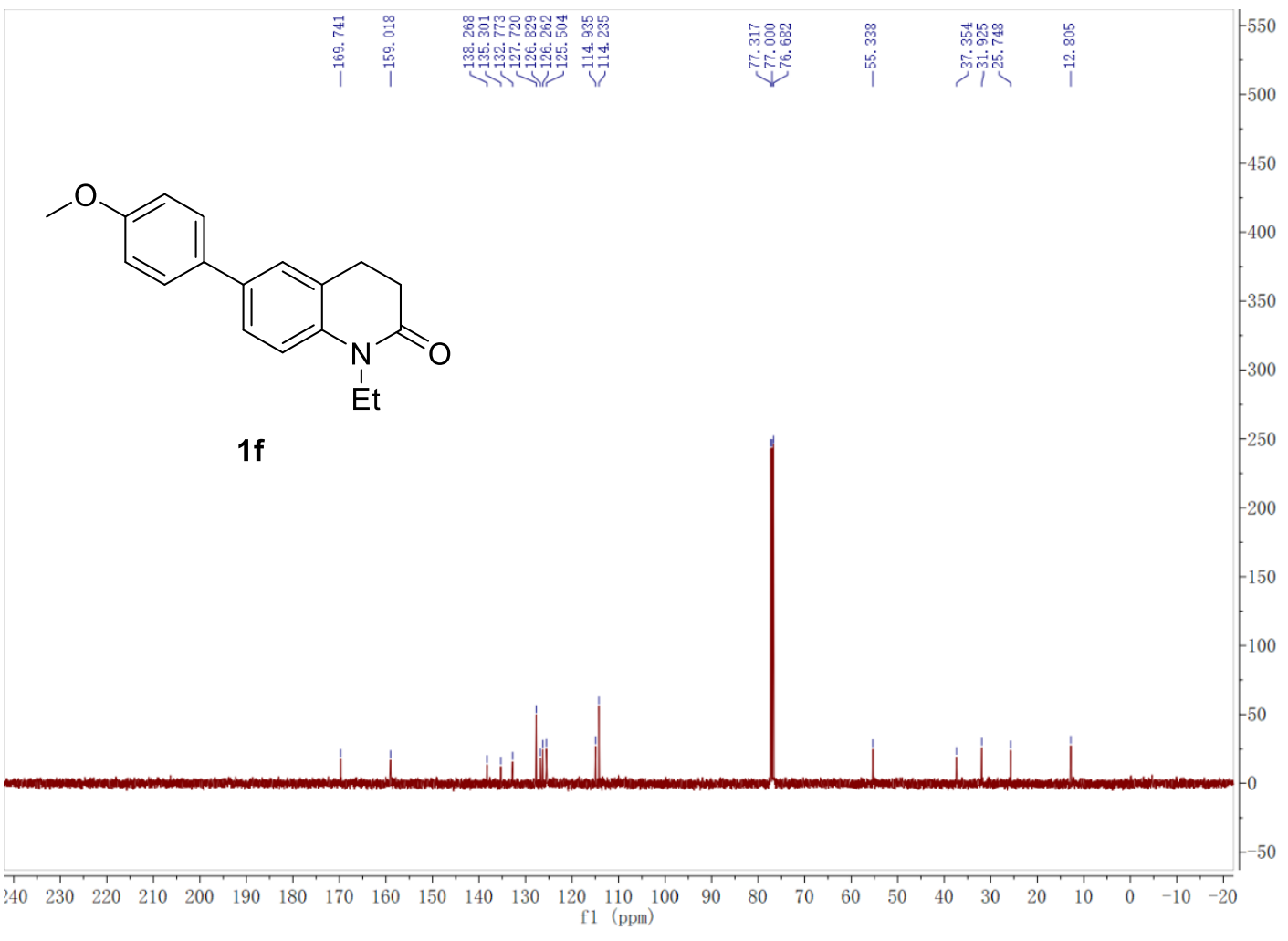

${ }^{13} \mathrm{C}$ NMR spectra $\left(101 \mathrm{MHz}, \mathrm{CDCl}_{3}\right)$ of $\mathbf{1 f}$ 


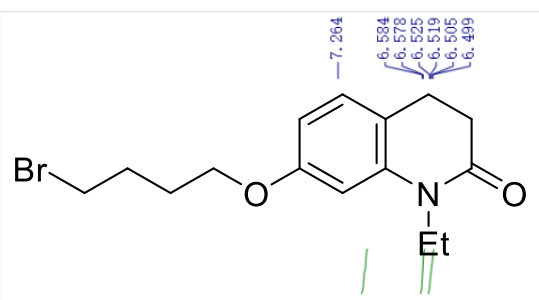

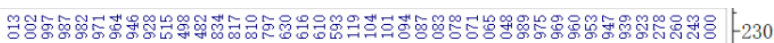
+

$1 \mathrm{~g}$
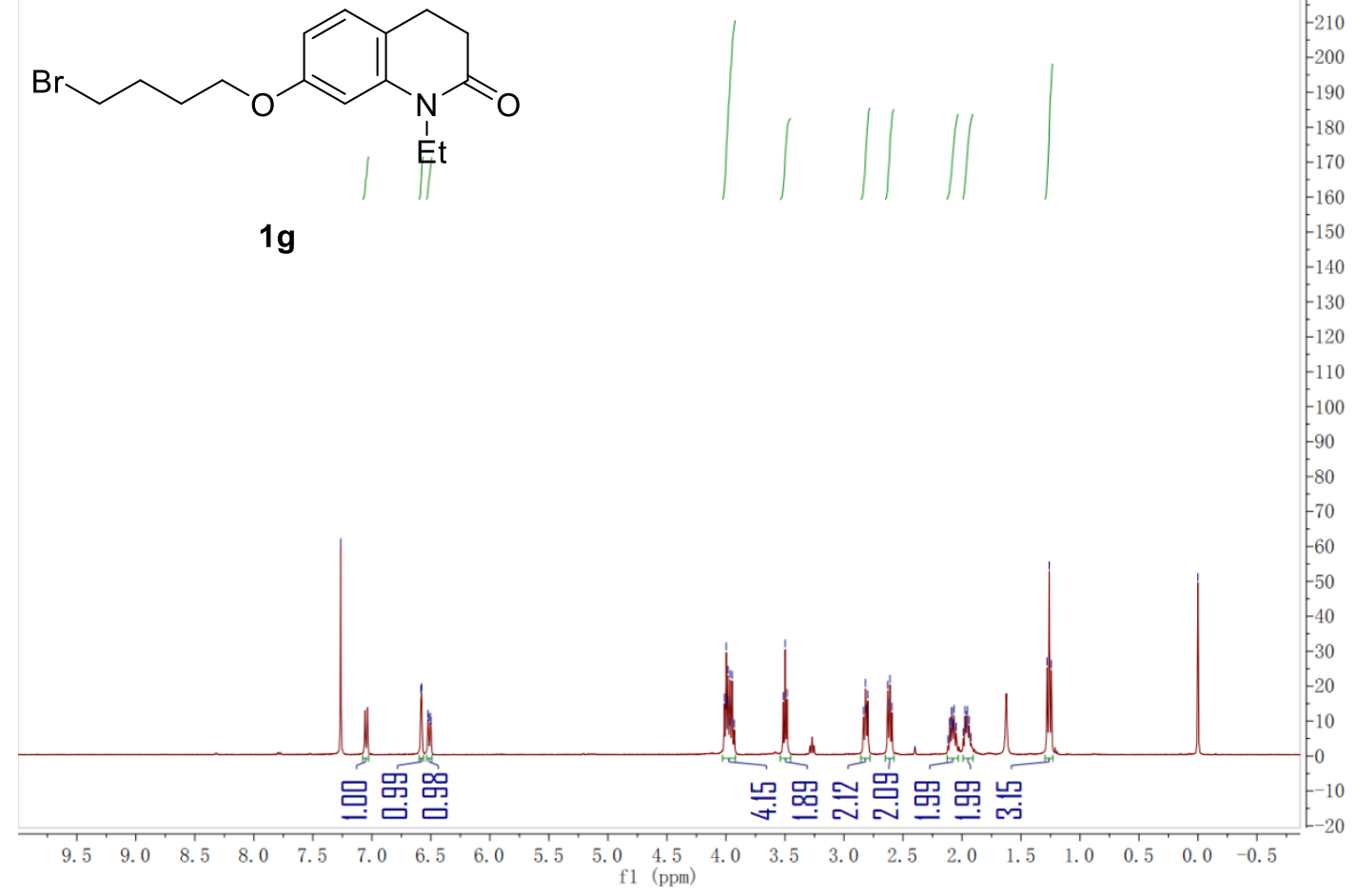

${ }^{1} \mathrm{H}$ NMR spectra $\left(400 \mathrm{MHz}, \mathrm{CDCl}_{3}\right)$ of $\mathbf{1 g}$

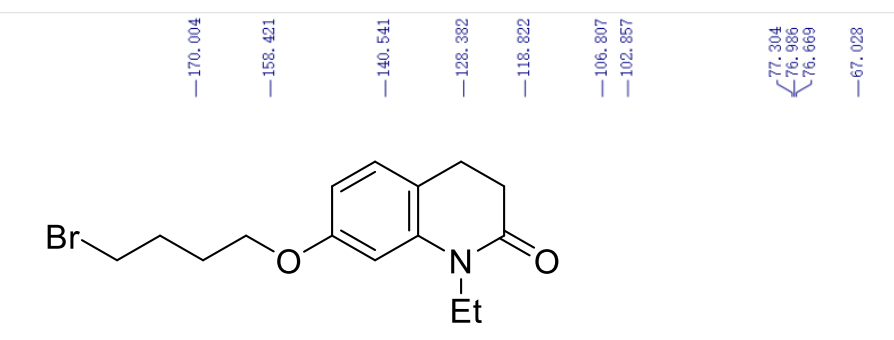

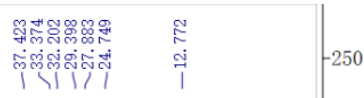

$1 \mathrm{~g}$

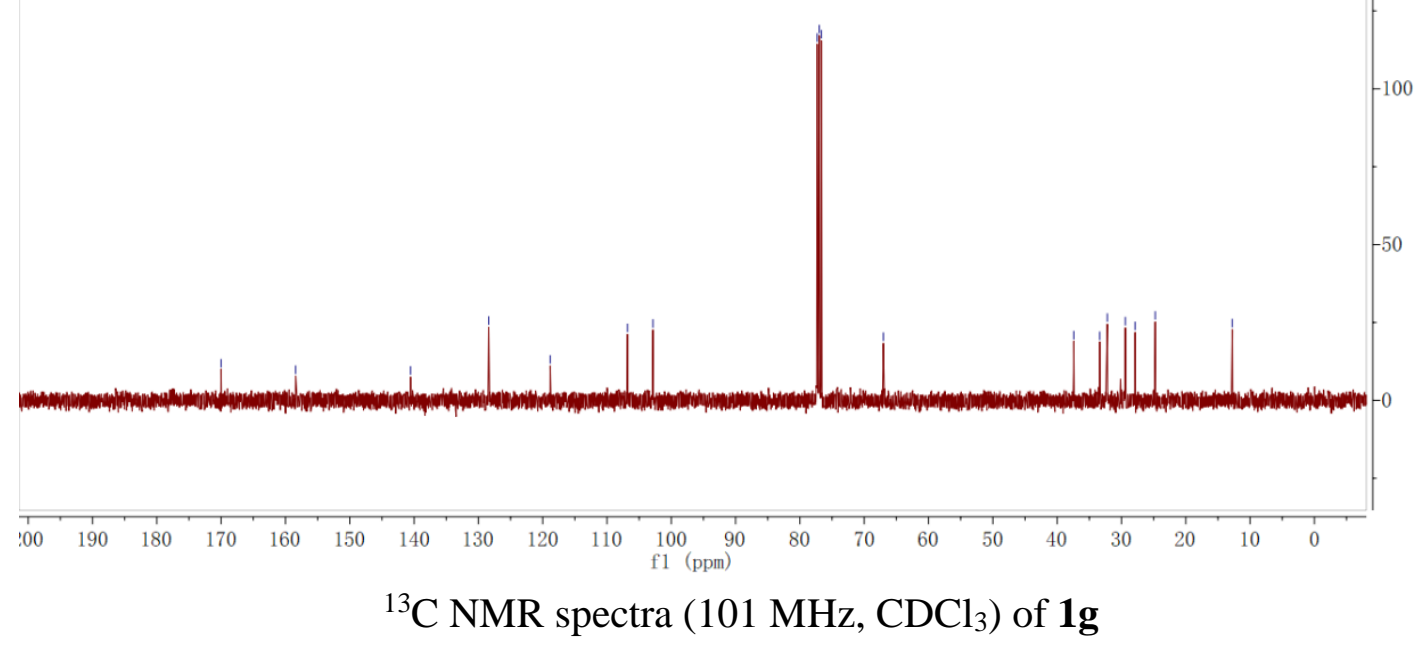




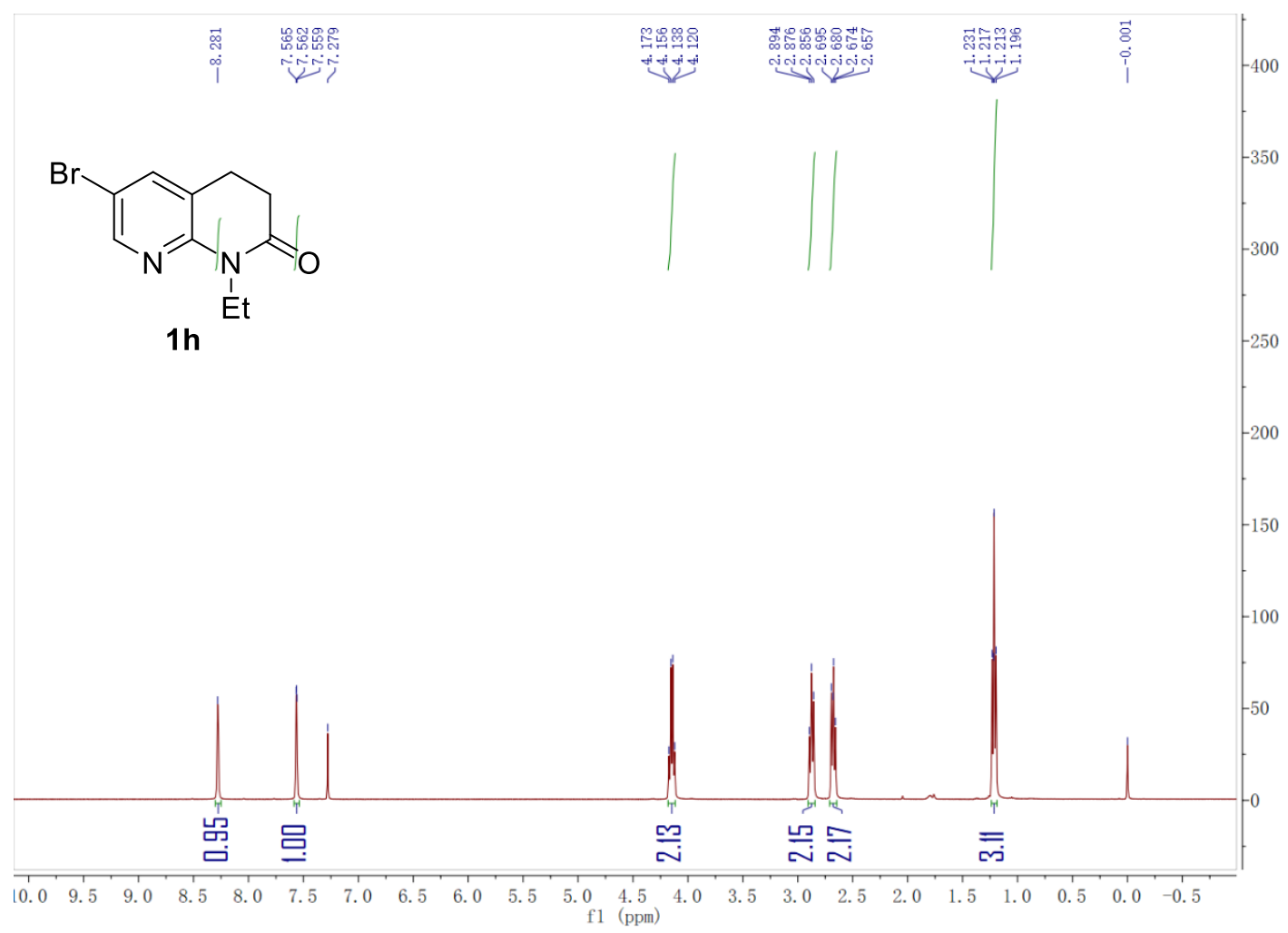

${ }^{1} \mathrm{H}$ NMR spectra $\left(400 \mathrm{MHz}, \mathrm{CDCl}_{3}\right)$ of $\mathbf{1 h}$

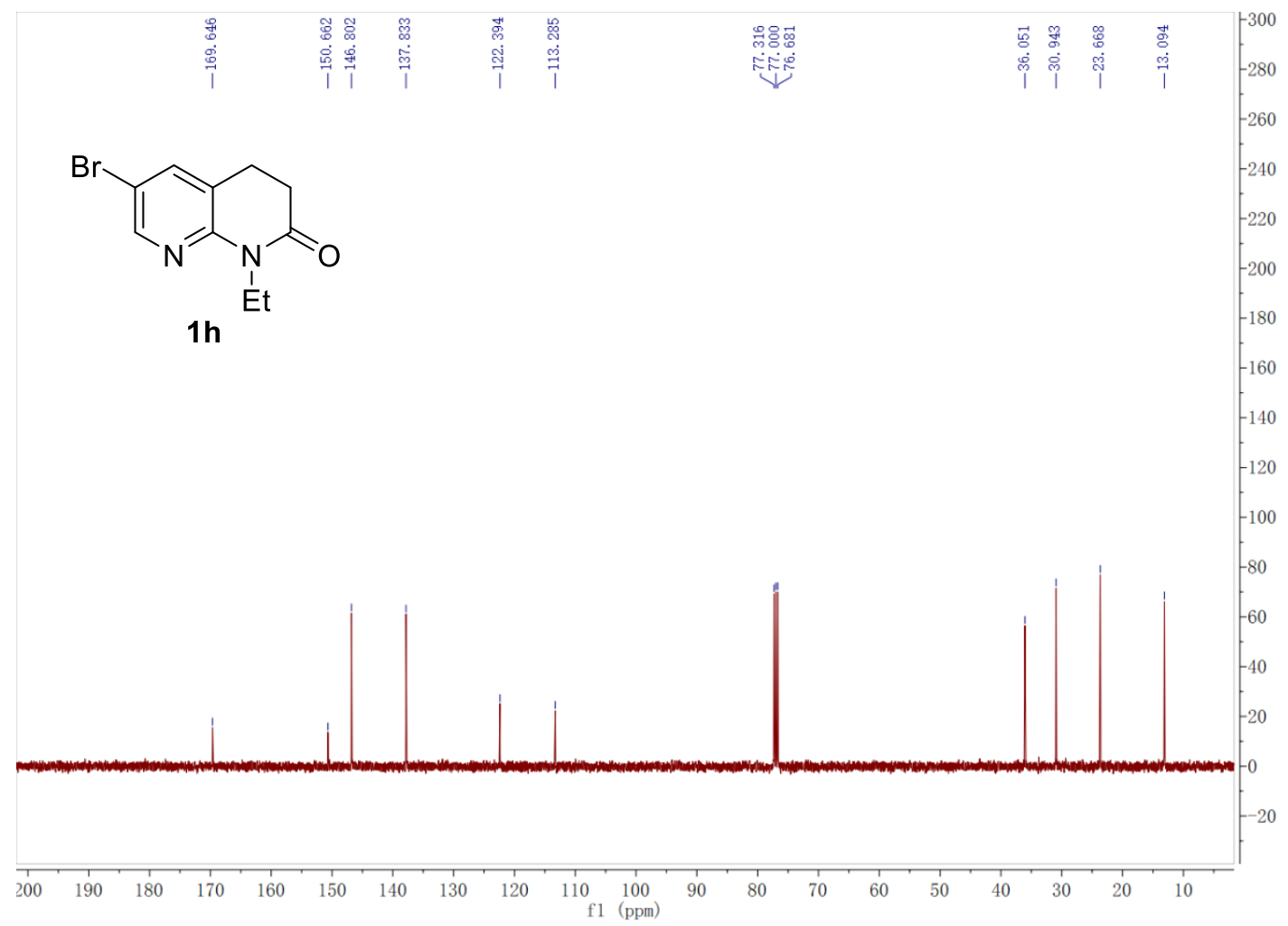

${ }^{13} \mathrm{C}$ NMR spectra $\left(101 \mathrm{MHz}, \mathrm{CDCl}_{3}\right)$ of $\mathbf{1 h}$ 


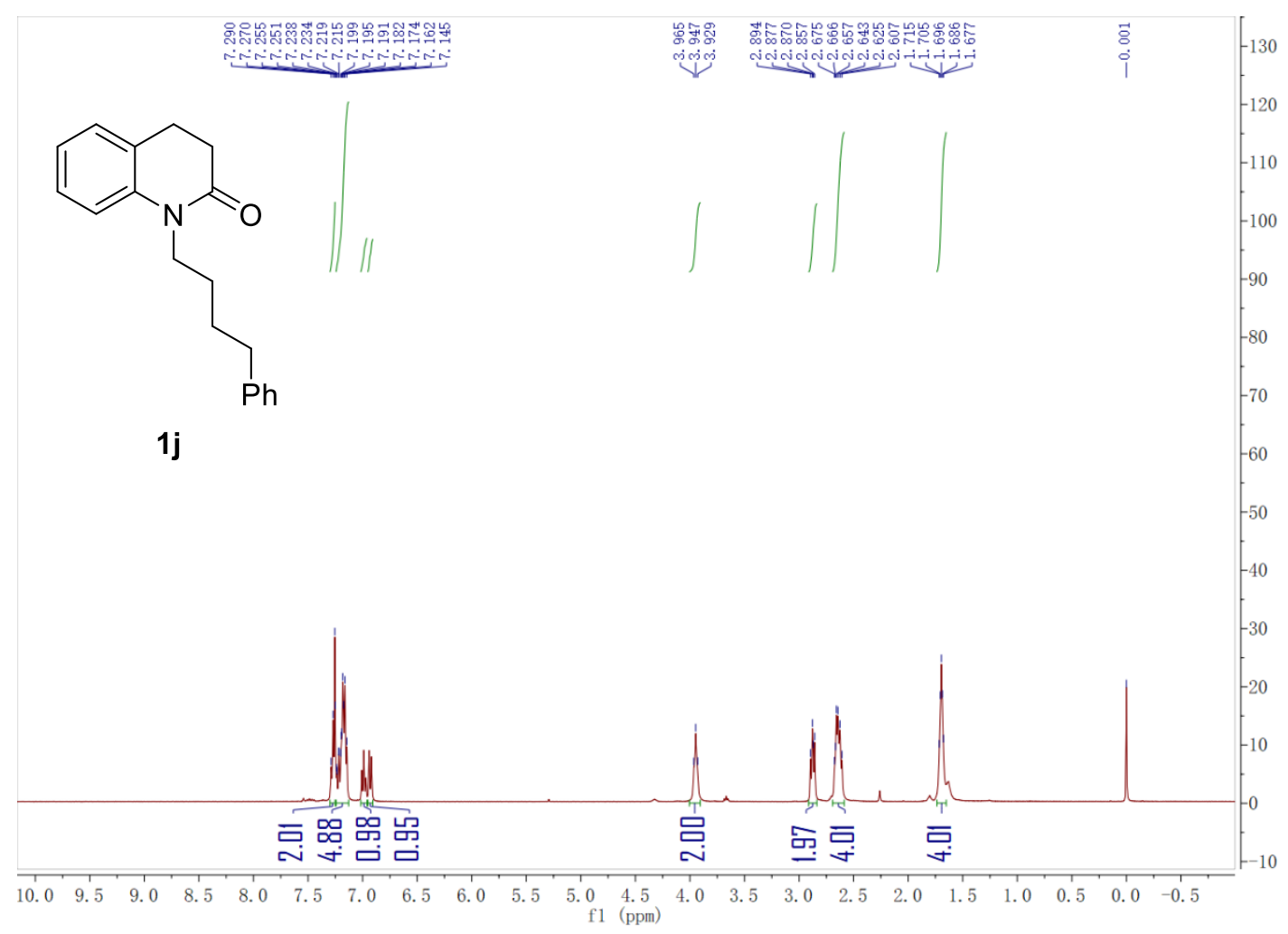

${ }^{1} \mathrm{H}$ NMR spectra $\left(400 \mathrm{MHz}, \mathrm{CDCl}_{3}\right)$ of $\mathbf{1 j}$

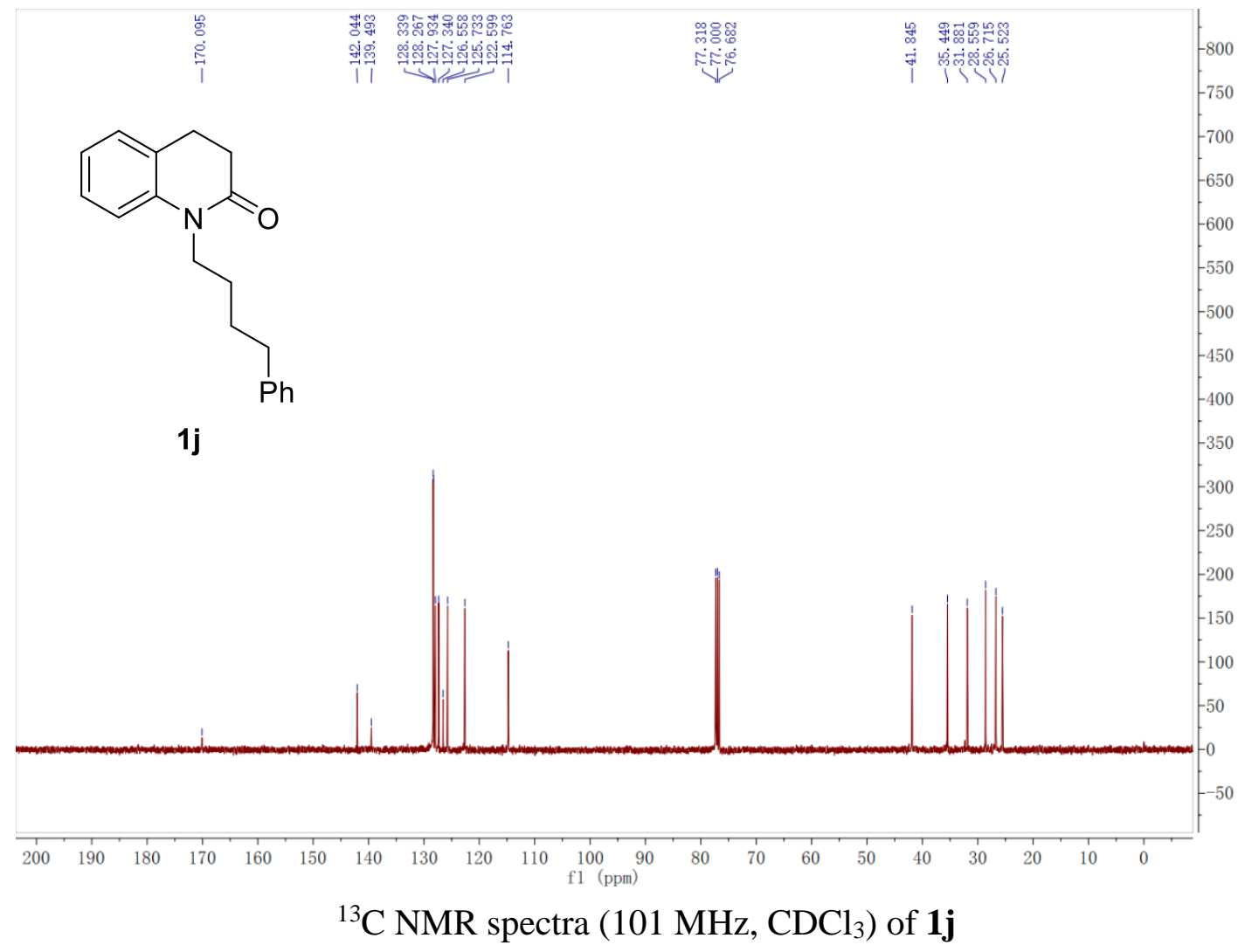




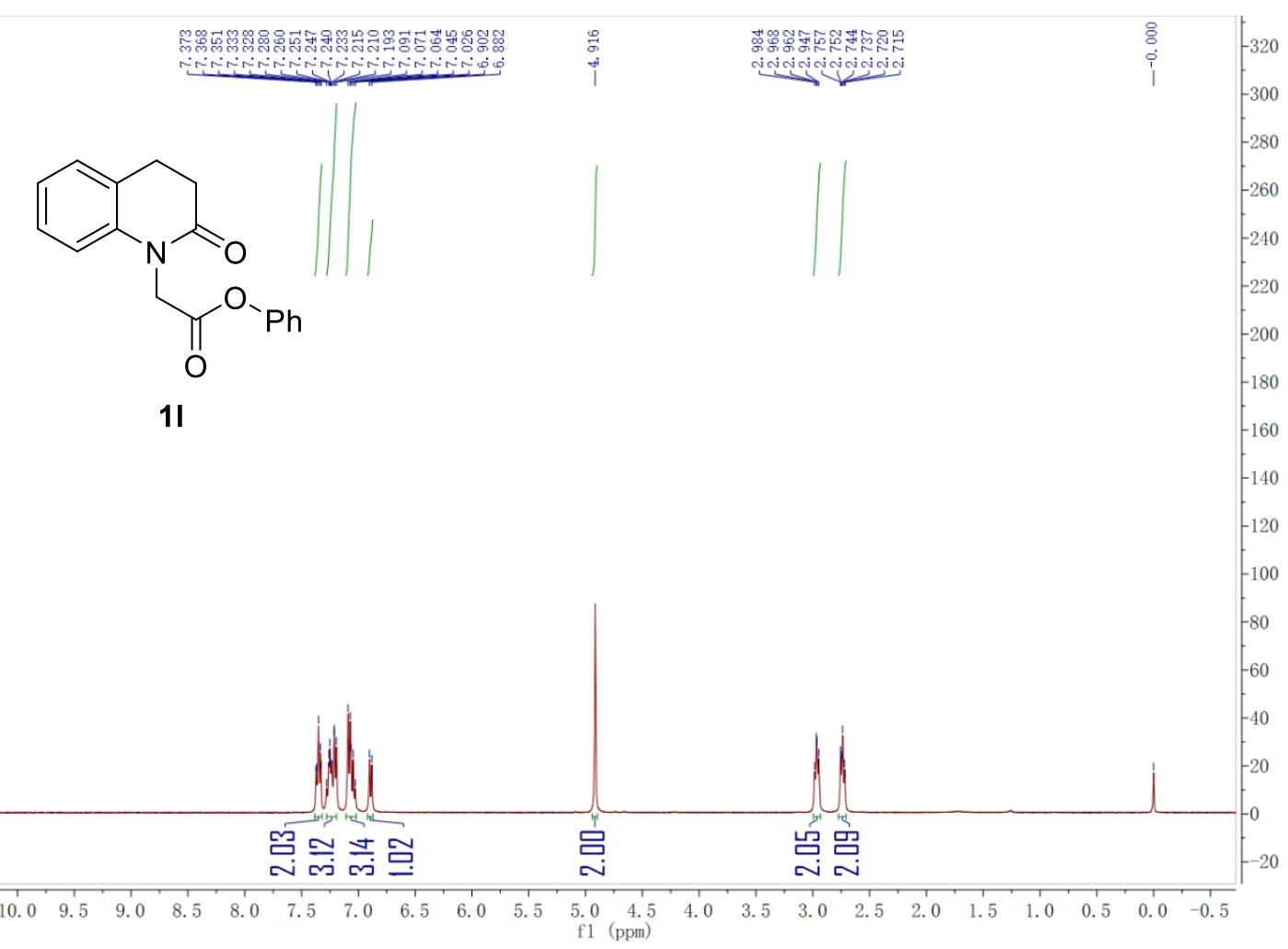

${ }^{1} \mathrm{H}$ NMR spectra $\left(400 \mathrm{MHz}, \mathrm{CDCl}_{3}\right)$ of $\mathbf{1 l}$

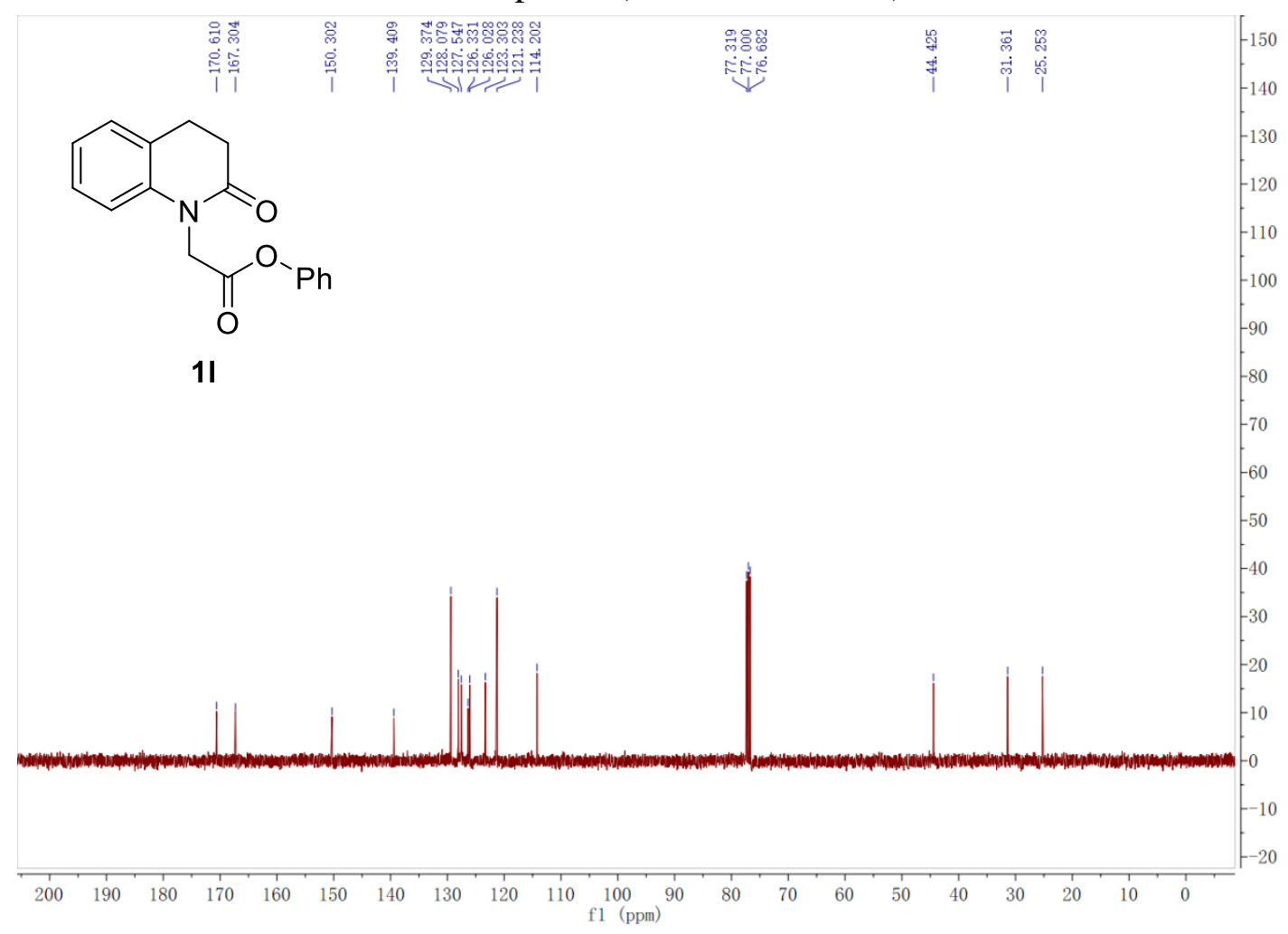

${ }^{13} \mathrm{C}$ NMR spectra $\left(101 \mathrm{MHz}, \mathrm{CDCl}_{3}\right)$ of $\mathbf{1 l}$ 


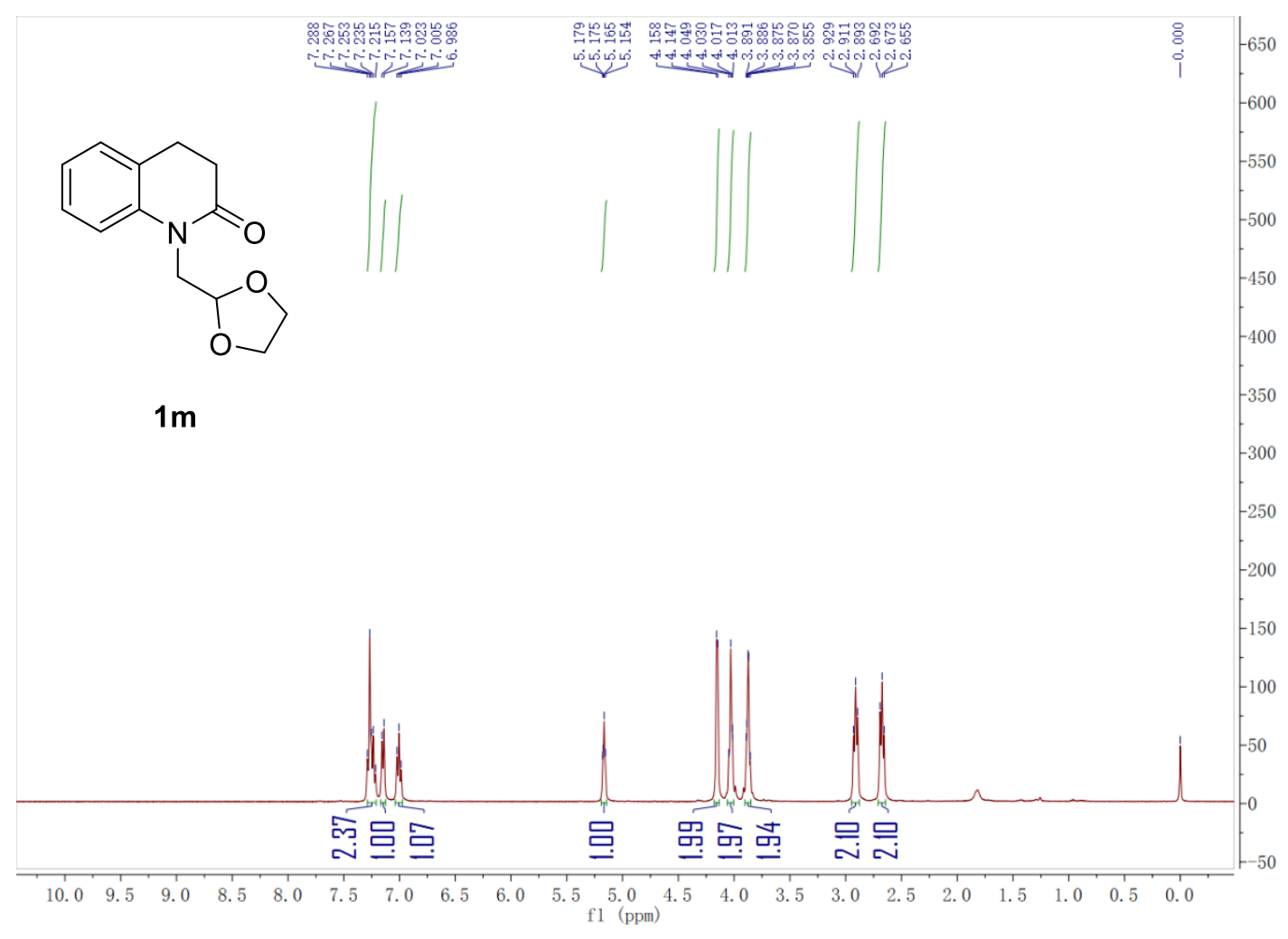

${ }^{1} \mathrm{H}$ NMR spectra $\left(400 \mathrm{MHz}, \mathrm{CDCl}_{3}\right)$ of $\mathbf{1 m}$

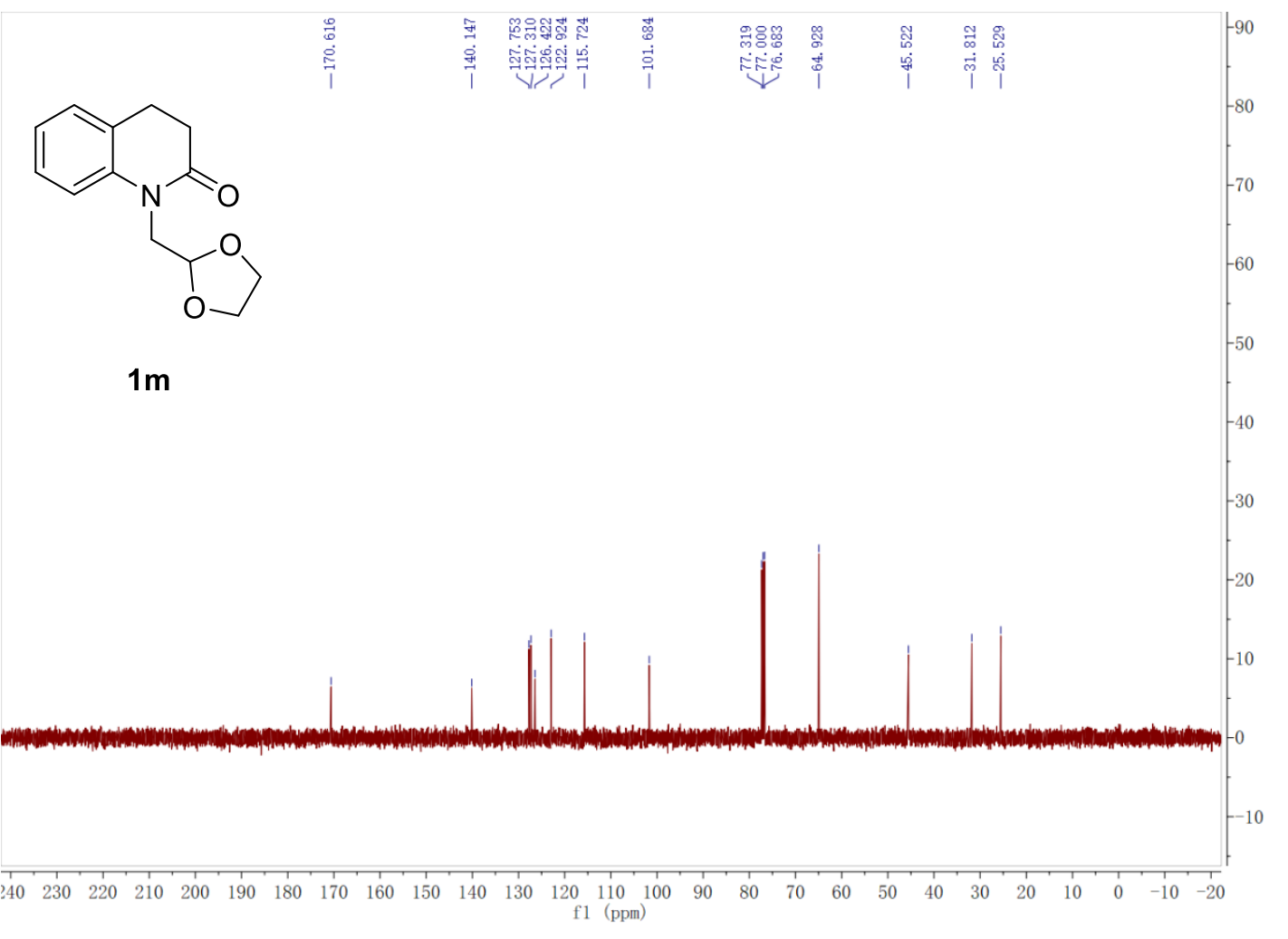

${ }^{13} \mathrm{C}$ NMR spectra $\left(101 \mathrm{MHz}, \mathrm{CDCl}_{3}\right)$ of $\mathbf{1 m}$ 


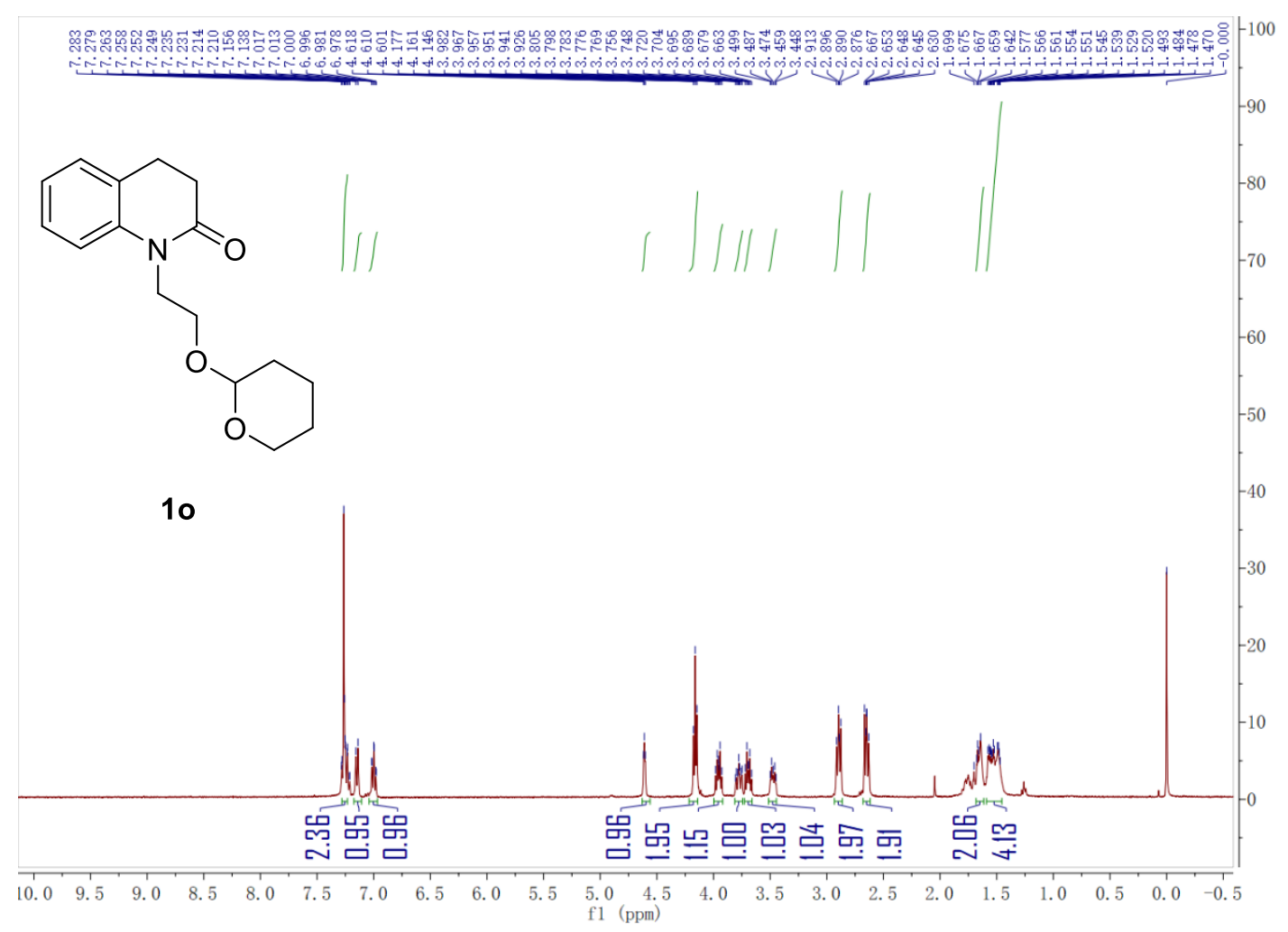

${ }^{1} \mathrm{H}$ NMR spectra (400 MHz, $\mathrm{CDCl}_{3}$ ) of $\mathbf{1 o}$

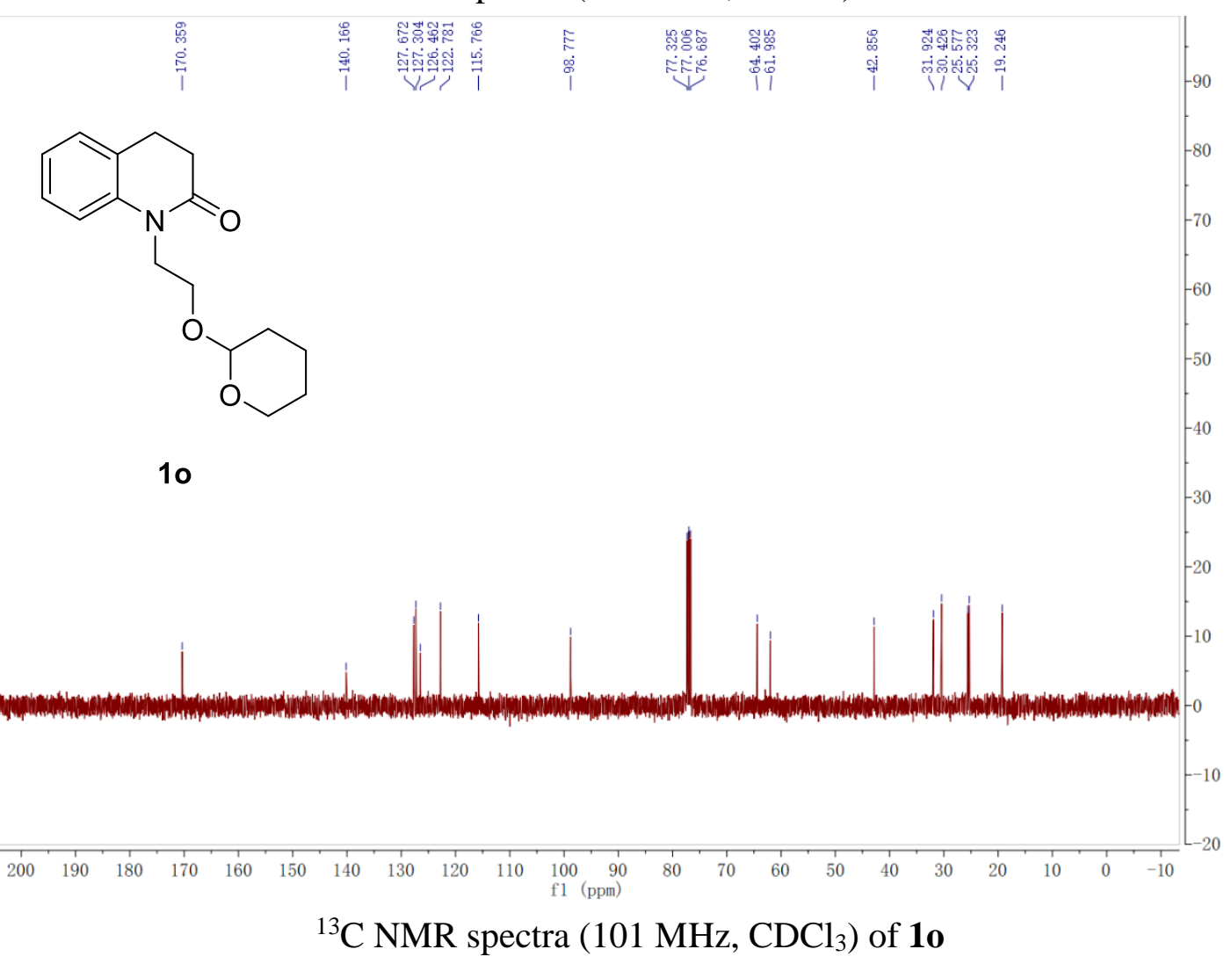




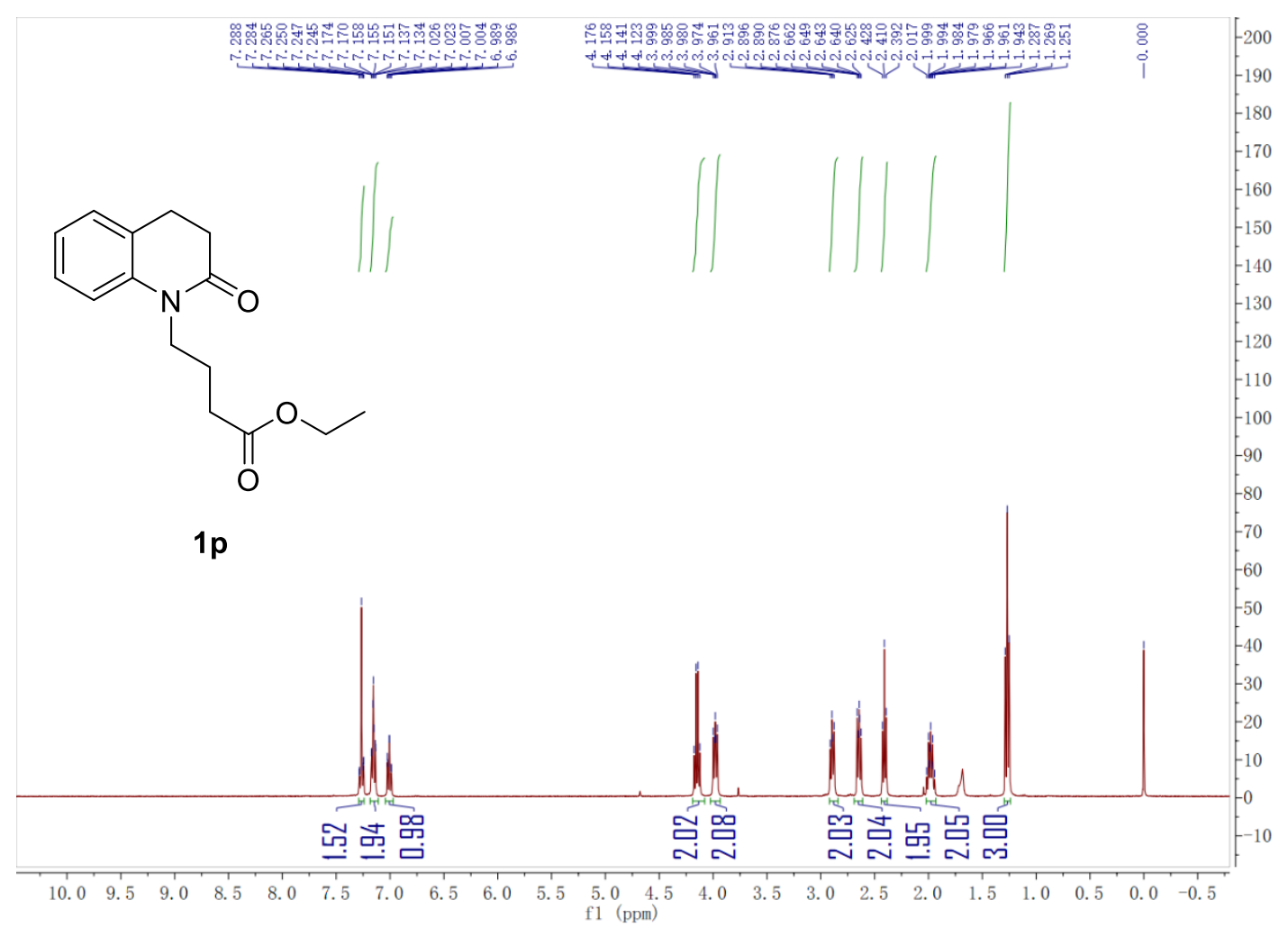

${ }^{1} \mathrm{H}$ NMR spectra $\left(400 \mathrm{MHz}, \mathrm{CDCl}_{3}\right)$ of $\mathbf{1 p}$

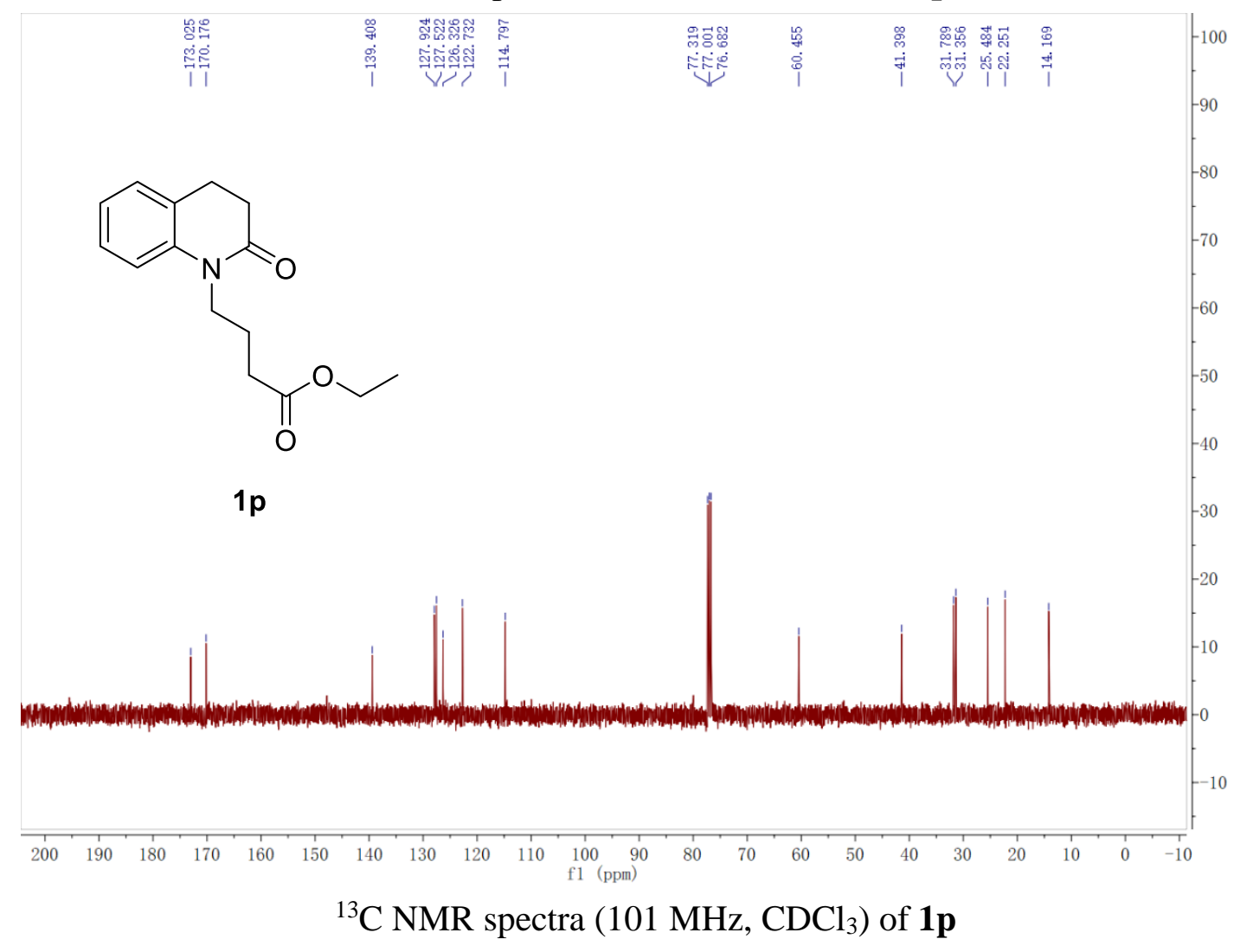




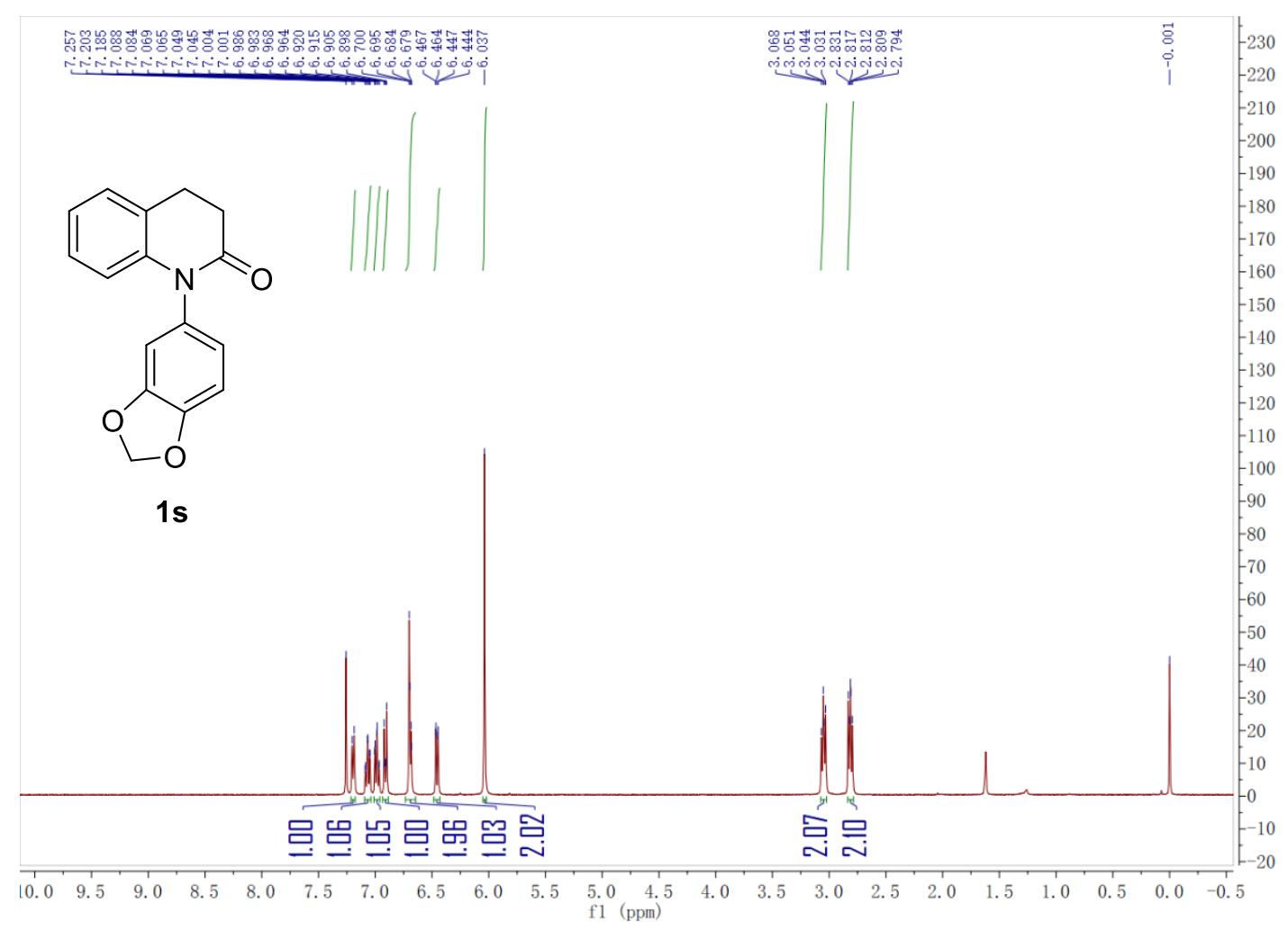

${ }^{1} \mathrm{H}$ NMR spectra (400 MHz, $\mathrm{CDCl}_{3}$ ) of $\mathbf{1 s}$

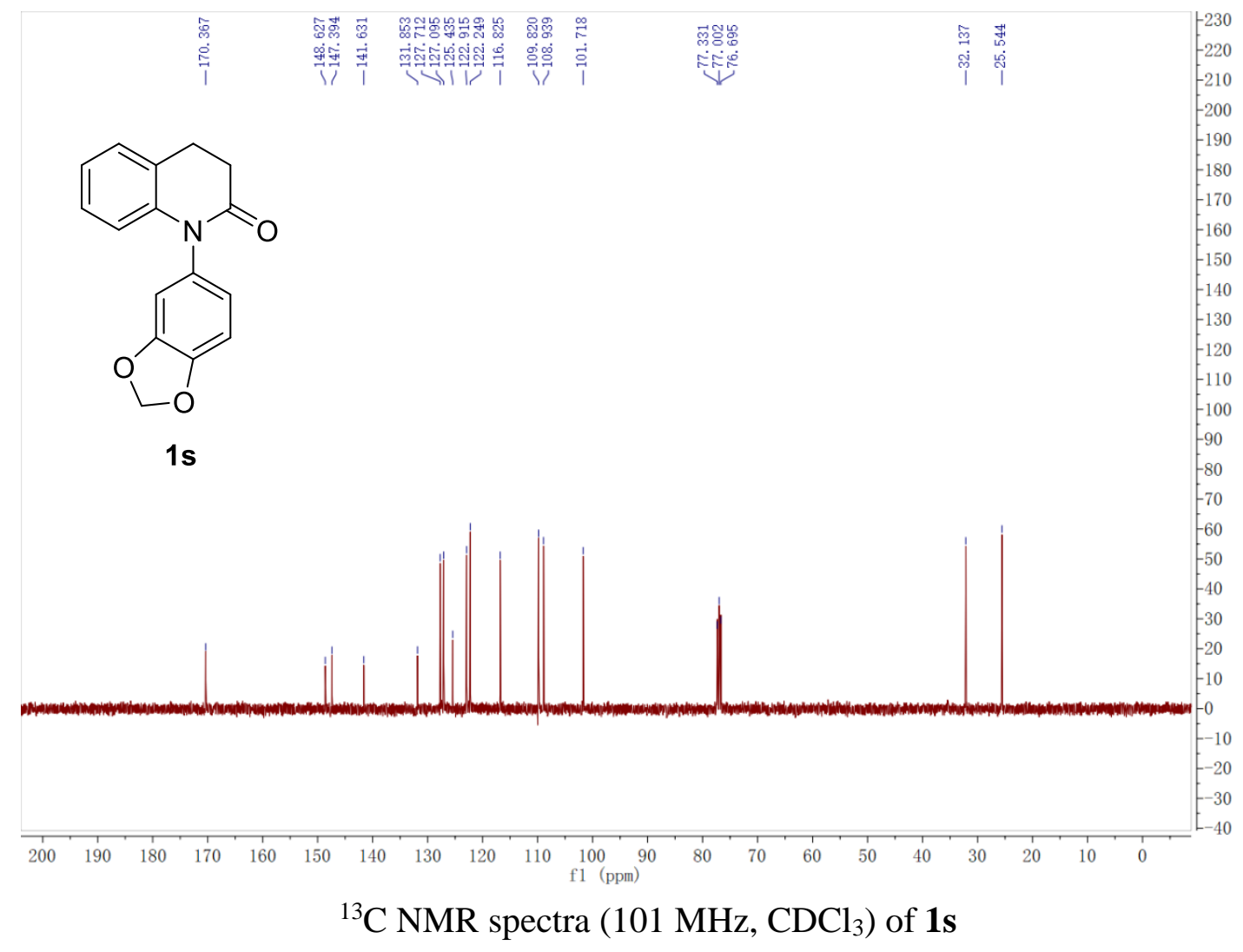




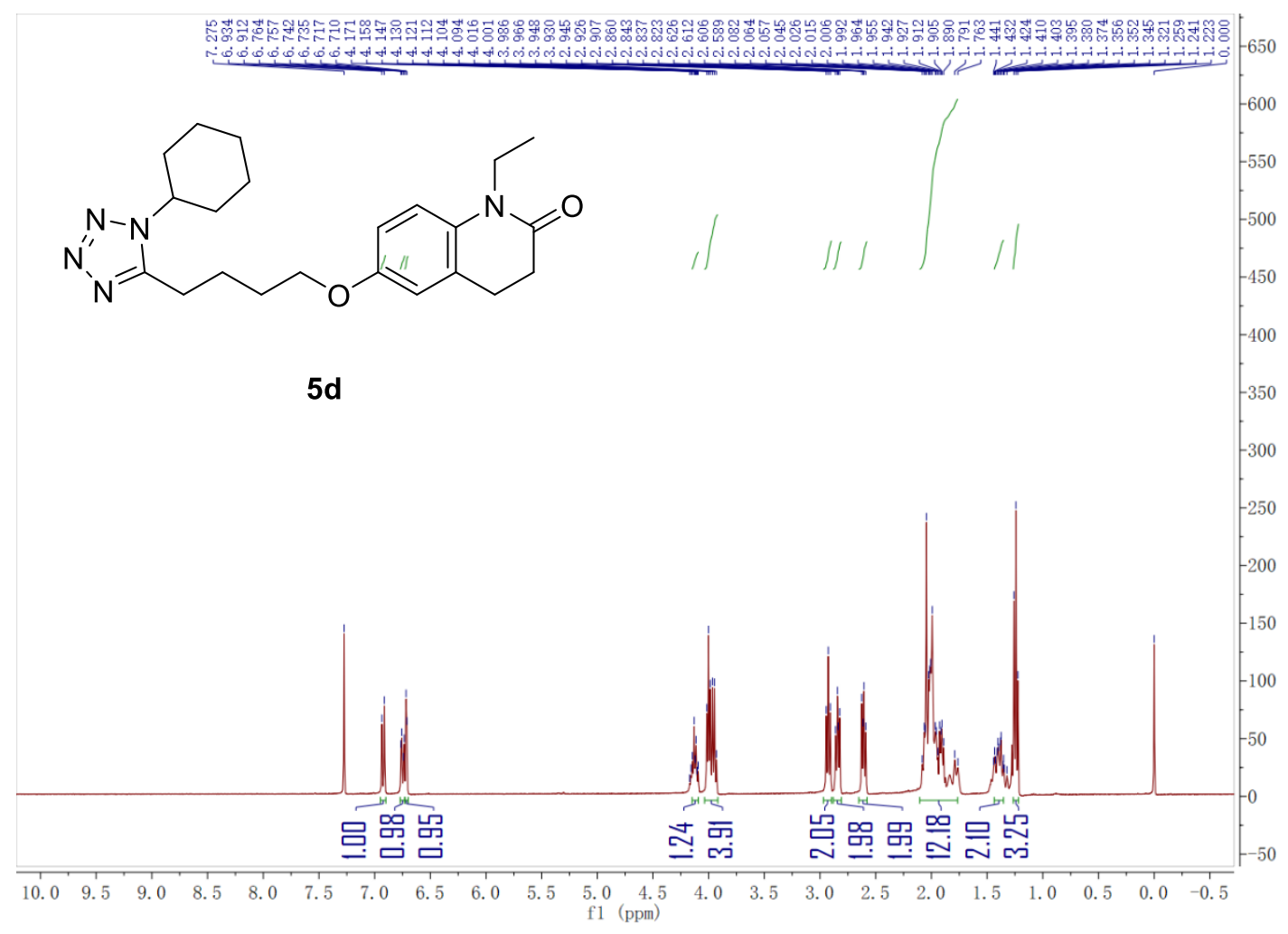

${ }^{1} \mathrm{H}$ NMR spectra $\left(400 \mathrm{MHz}, \mathrm{CDCl}_{3}\right)$ of $\mathbf{5 d}$

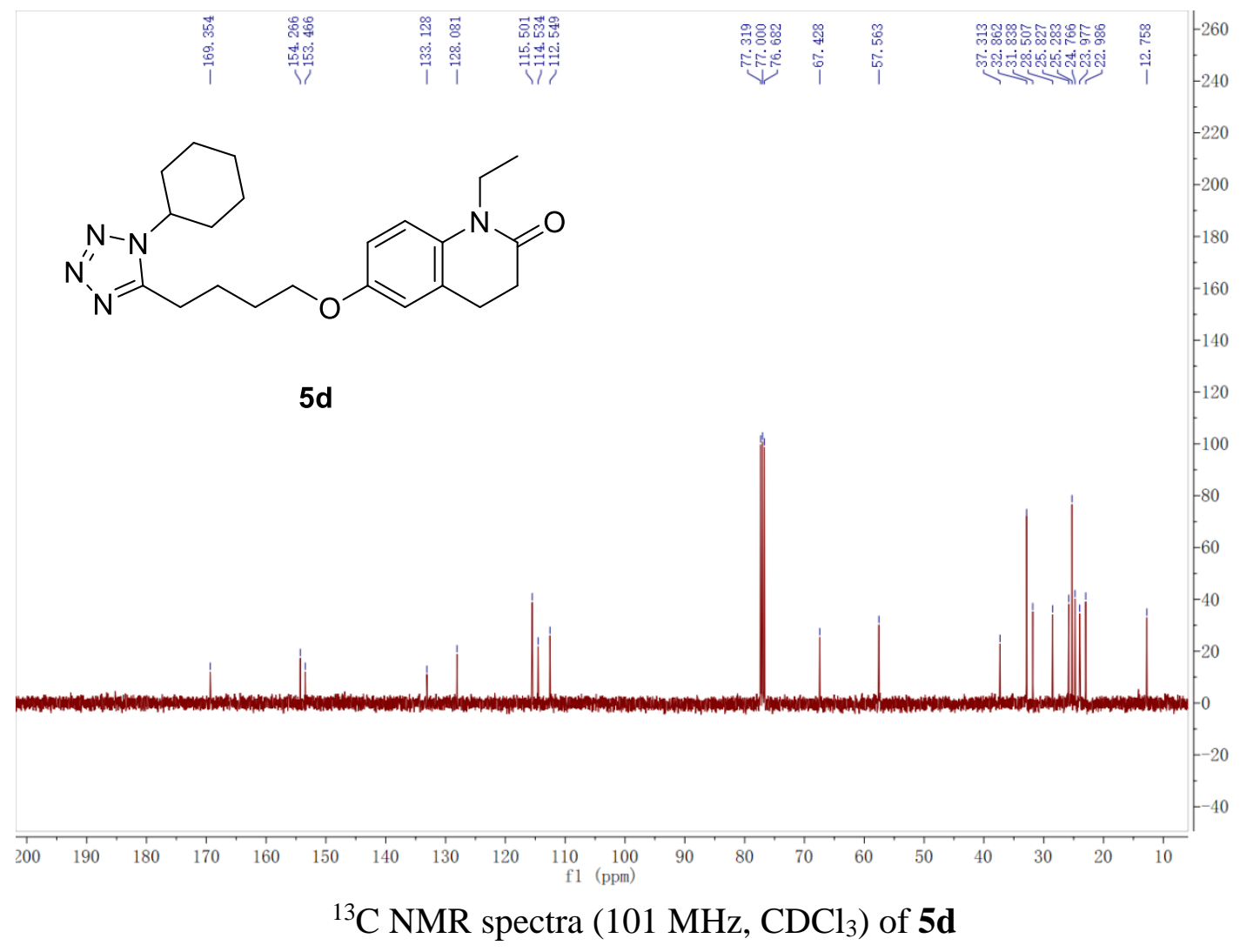




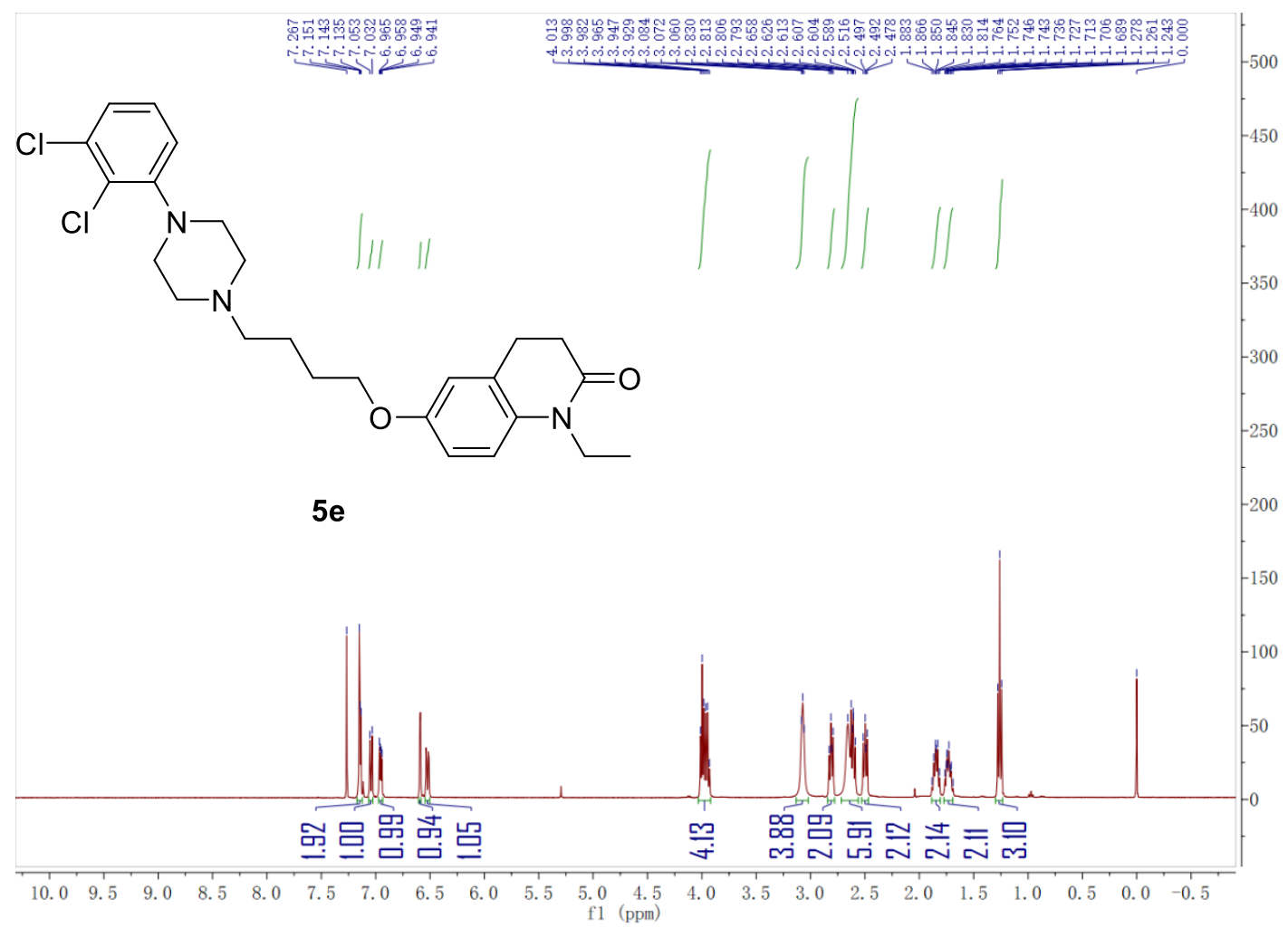

${ }^{1} \mathrm{H}$ NMR spectra $\left(400 \mathrm{MHz}, \mathrm{CDCl}_{3}\right)$ of $\mathbf{5 e}$

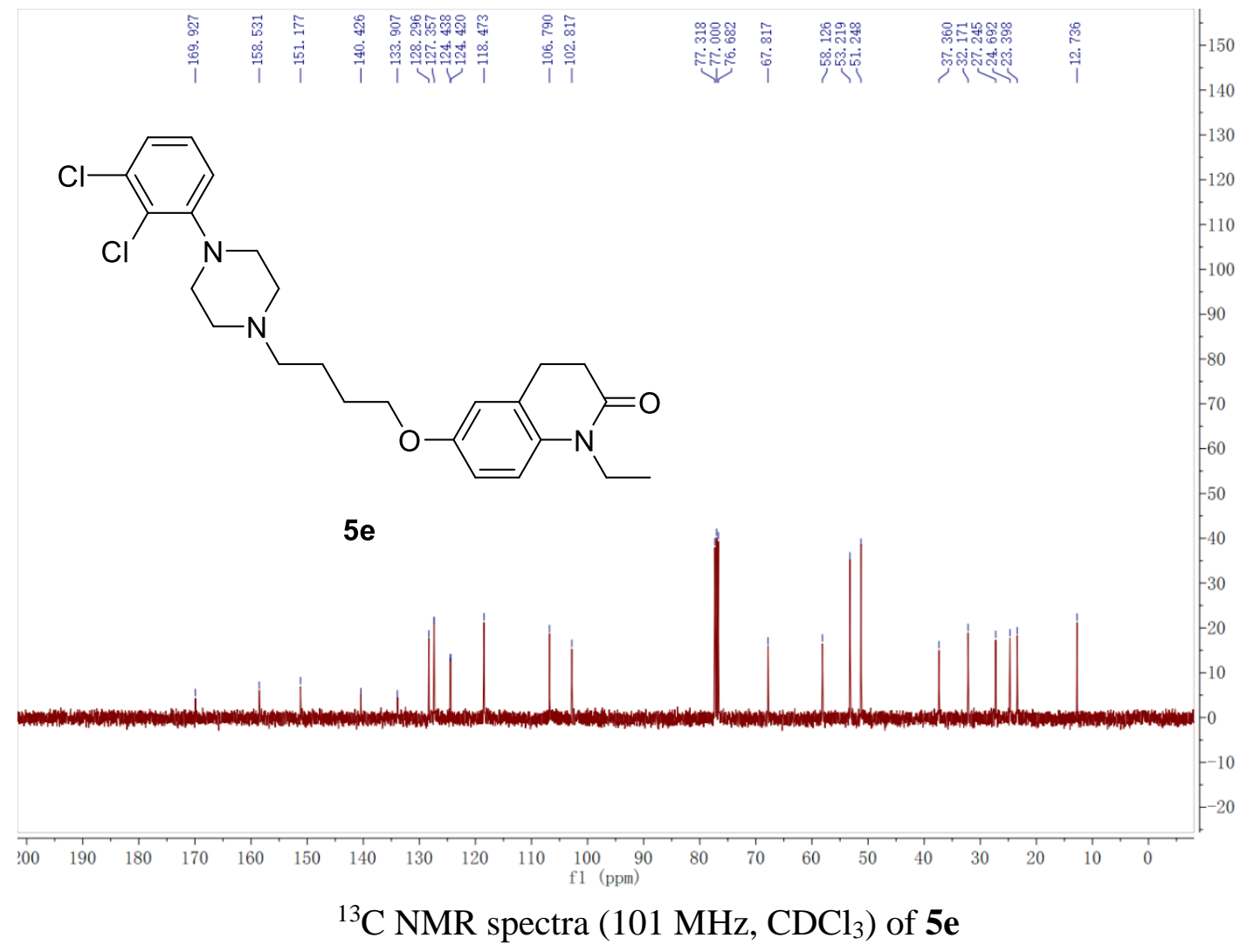



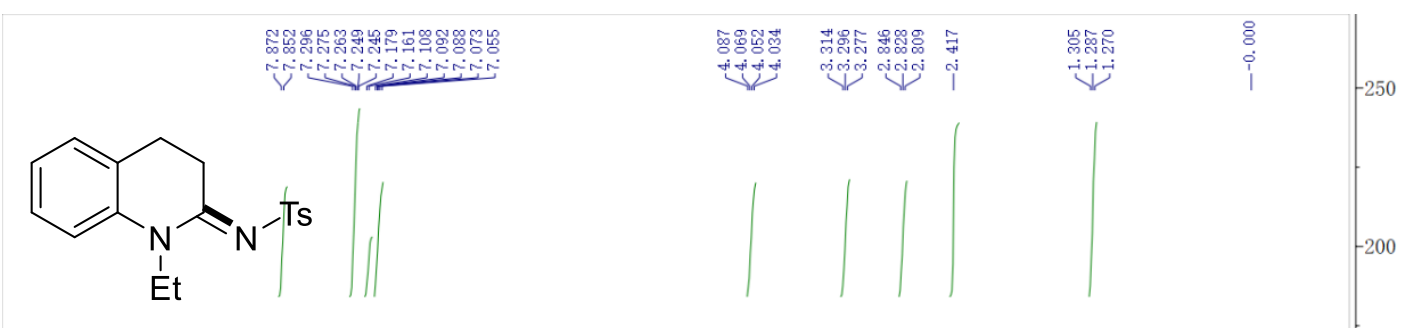

3a

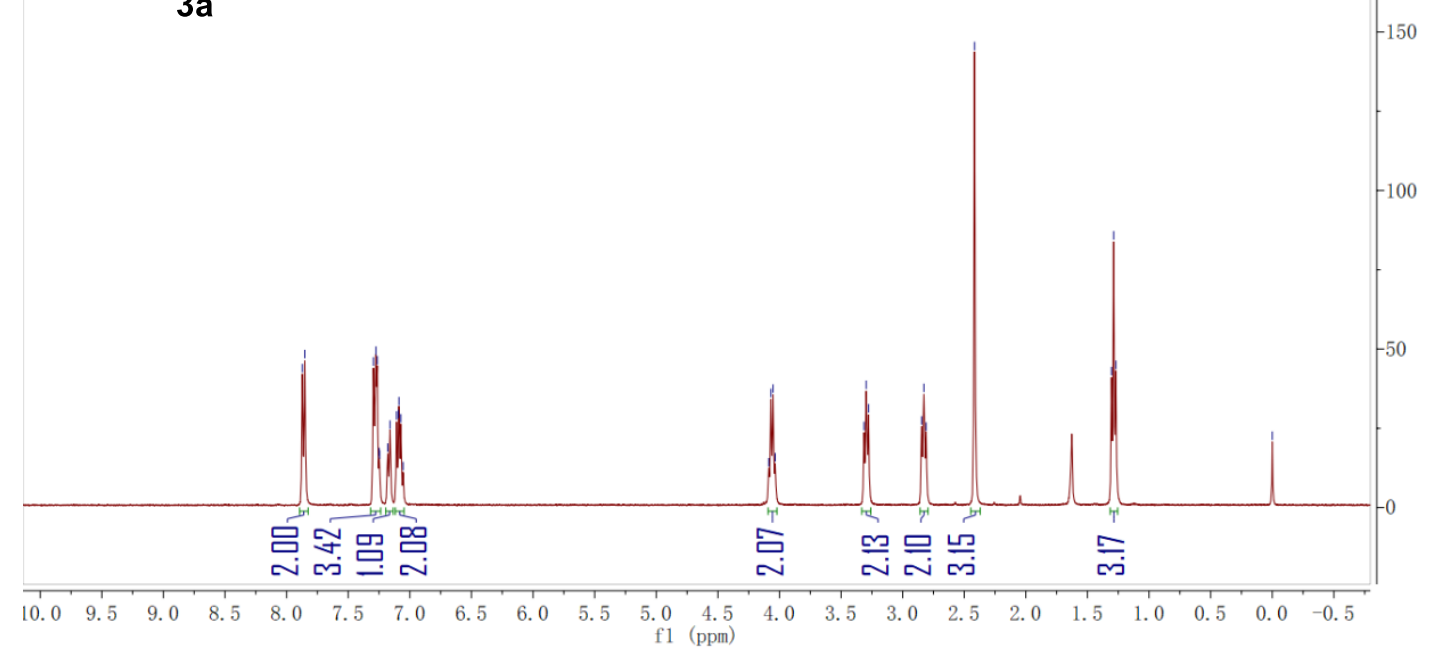

${ }^{1} \mathrm{H}$ NMR spectra $\left(400 \mathrm{MHz}, \mathrm{CDCl}_{3}\right)$ of $\mathbf{3 a}$

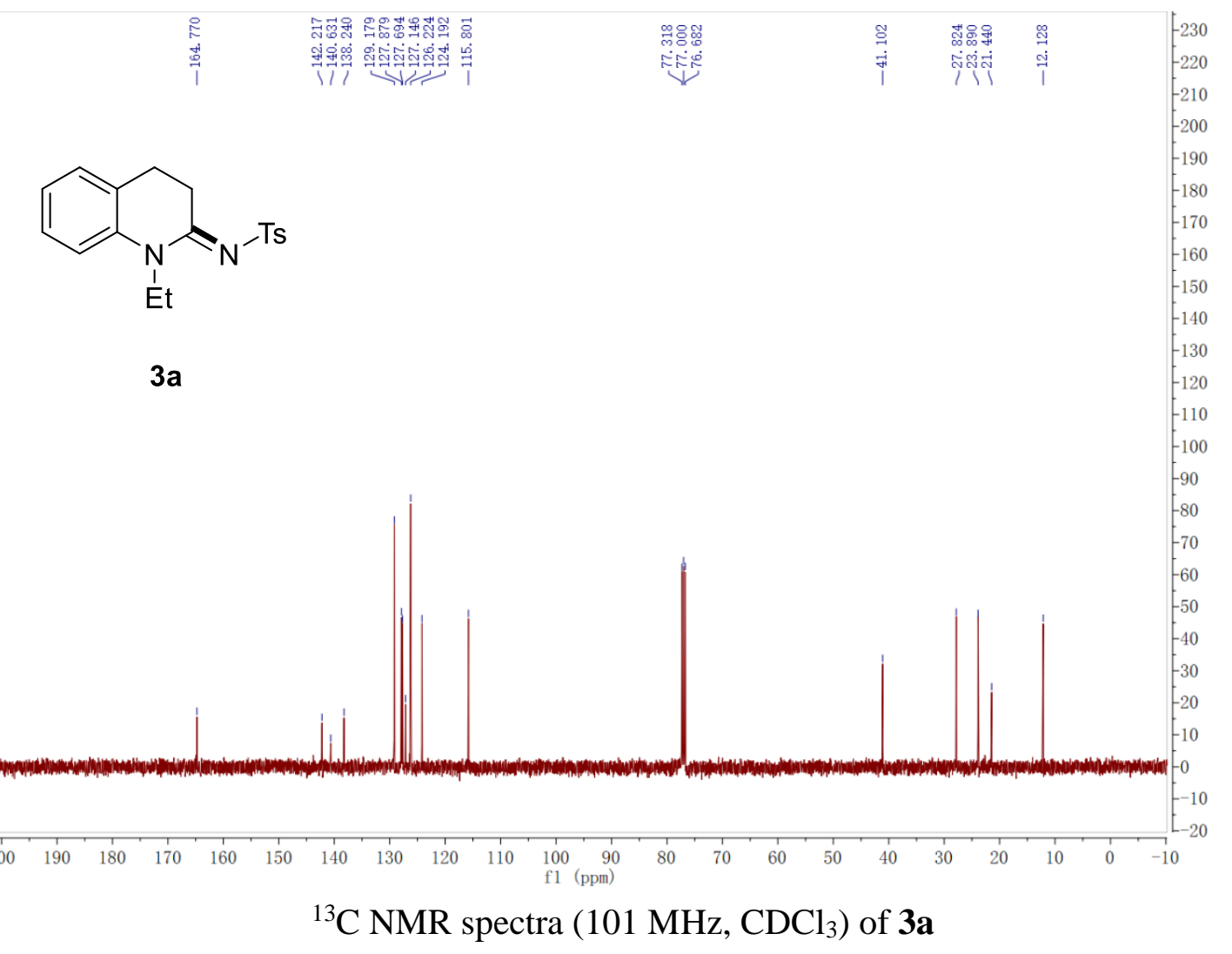




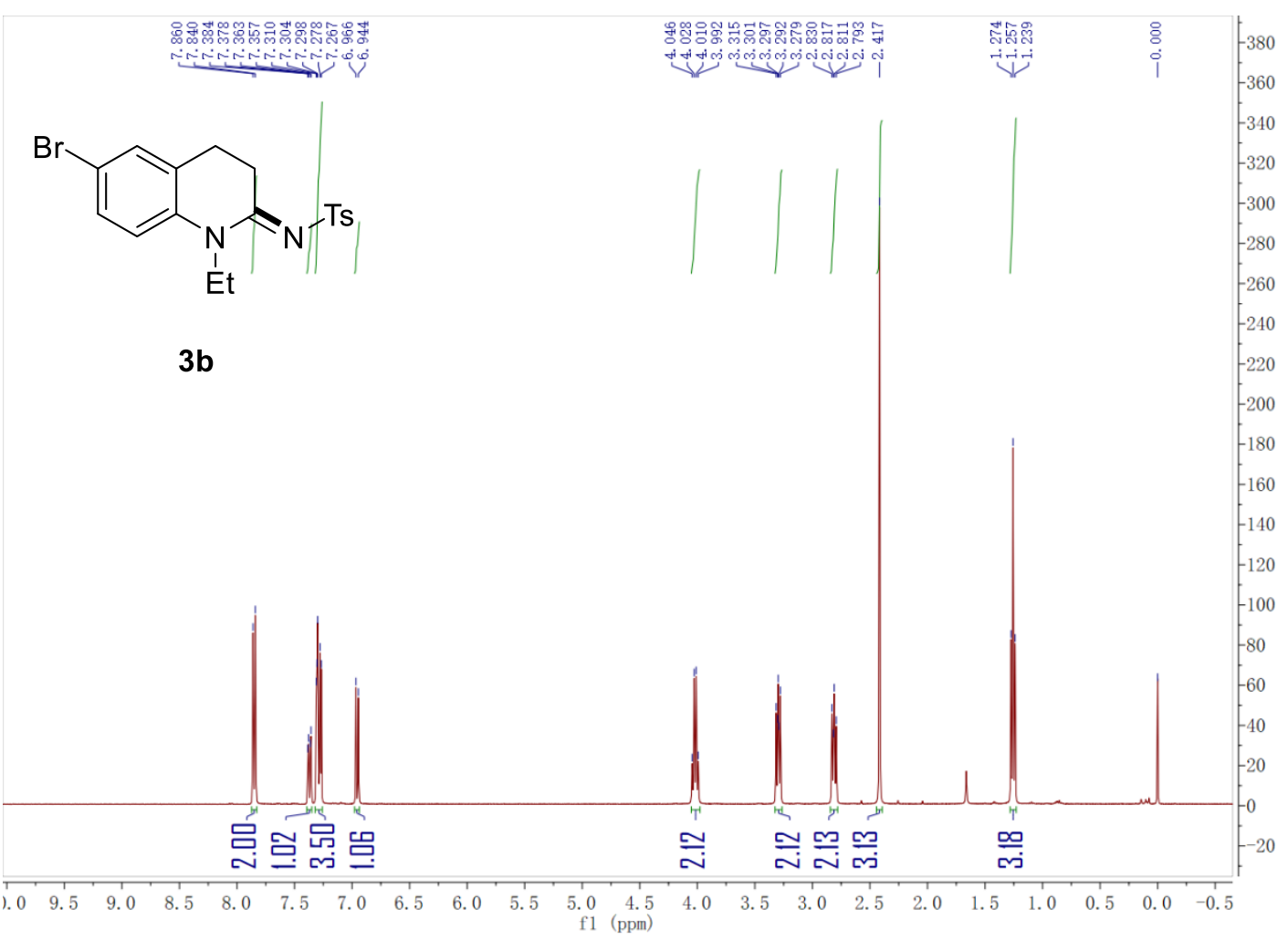

${ }^{1} \mathrm{H}$ NMR spectra $\left(400 \mathrm{MHz}, \mathrm{CDCl}_{3}\right)$ of $\mathbf{3 b}$

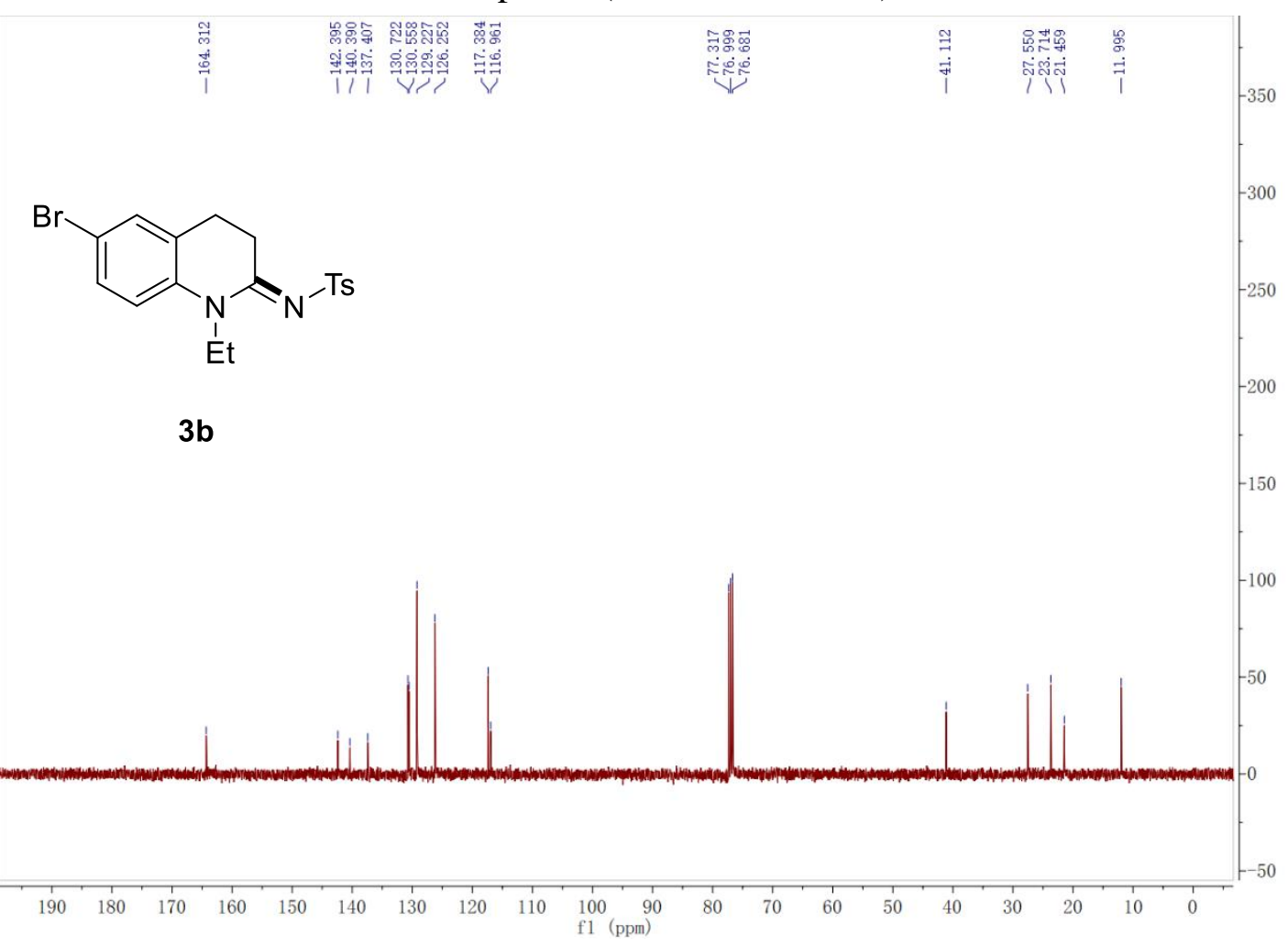

${ }^{13} \mathrm{C}$ NMR spectra $\left(101 \mathrm{MHz}, \mathrm{CDCl}_{3}\right)$ of $\mathbf{3 b}$ 


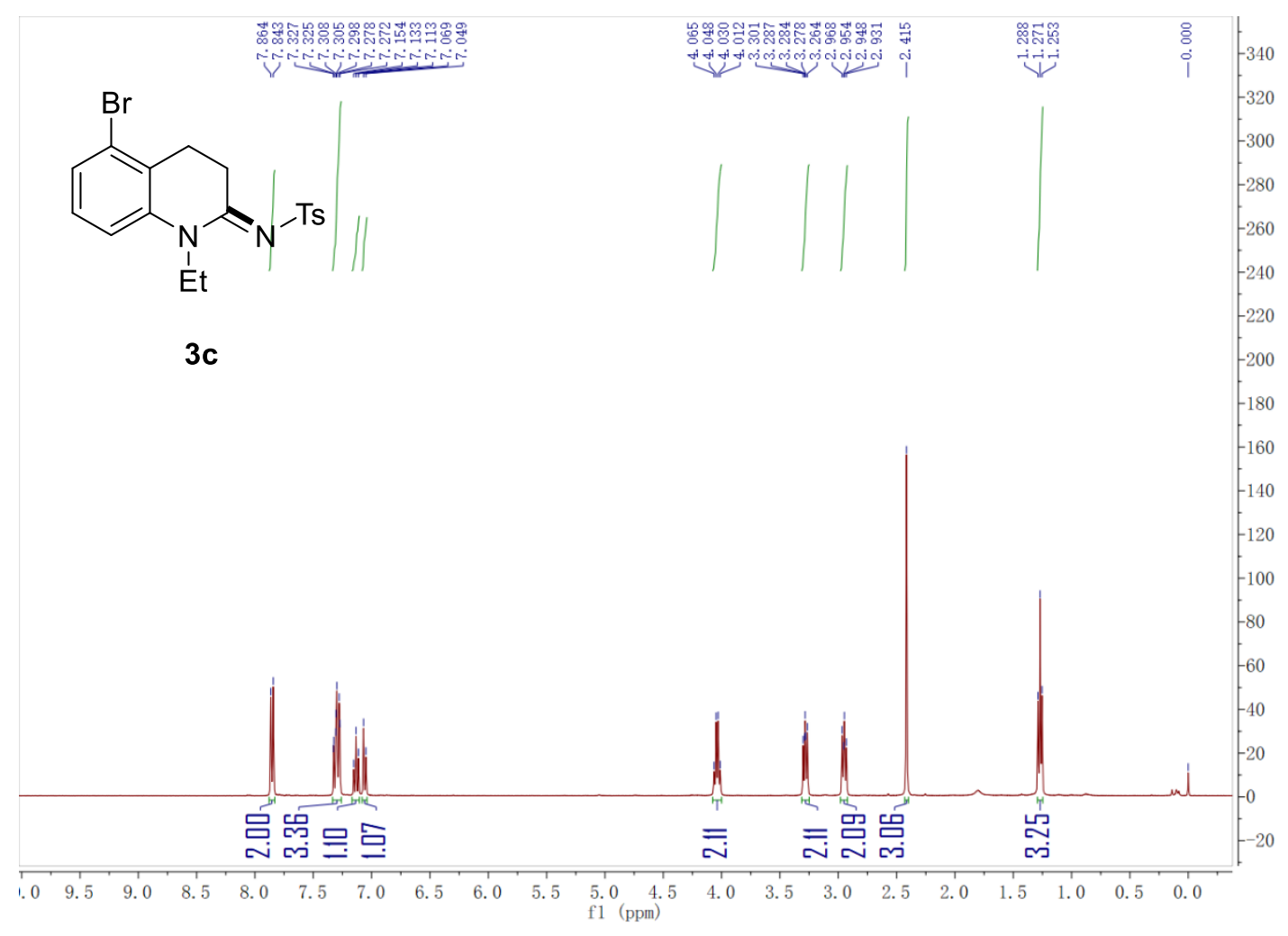

${ }^{1} \mathrm{H}$ NMR spectra $\left(400 \mathrm{MHz}, \mathrm{CDCl}_{3}\right)$ of $\mathbf{3 c}$

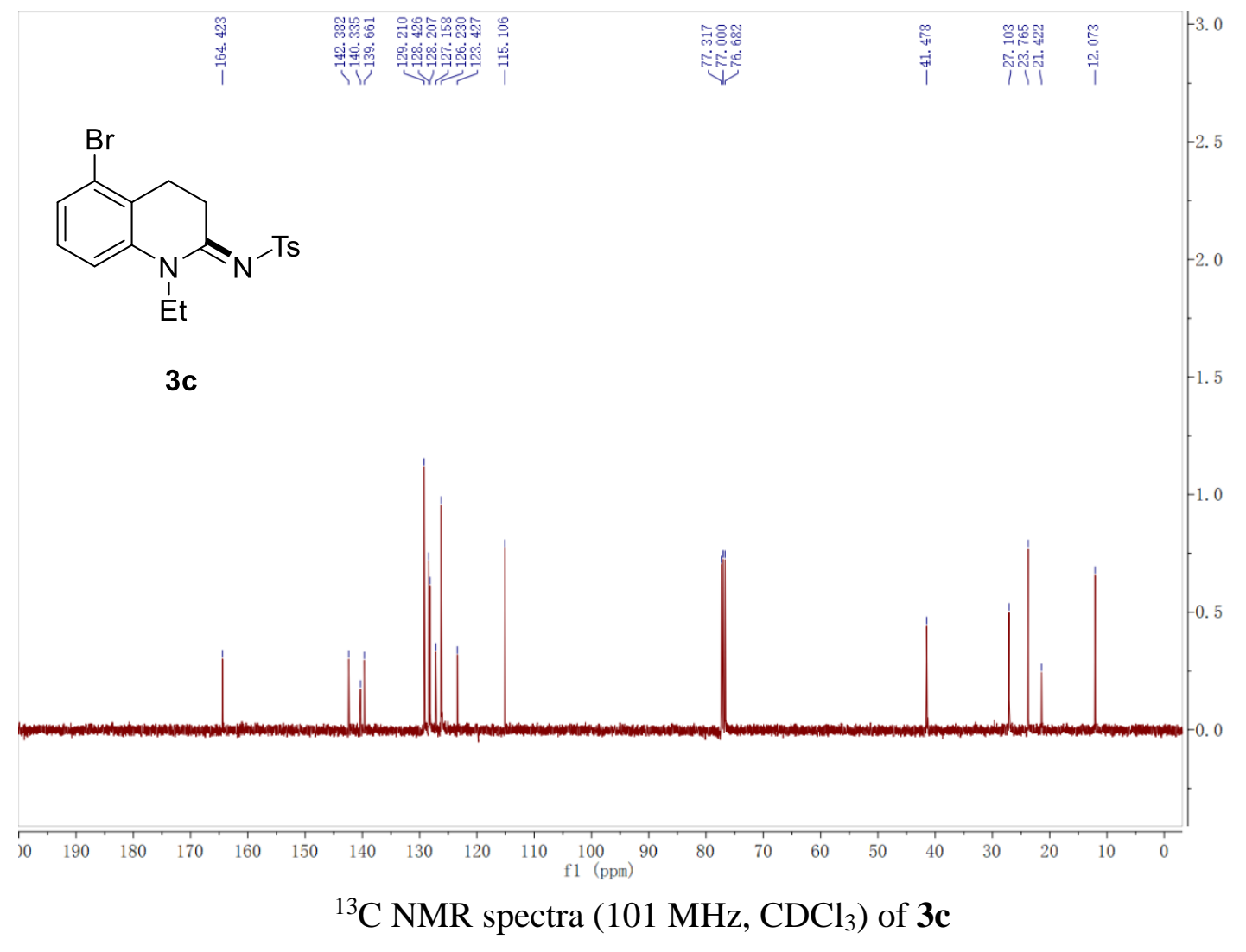




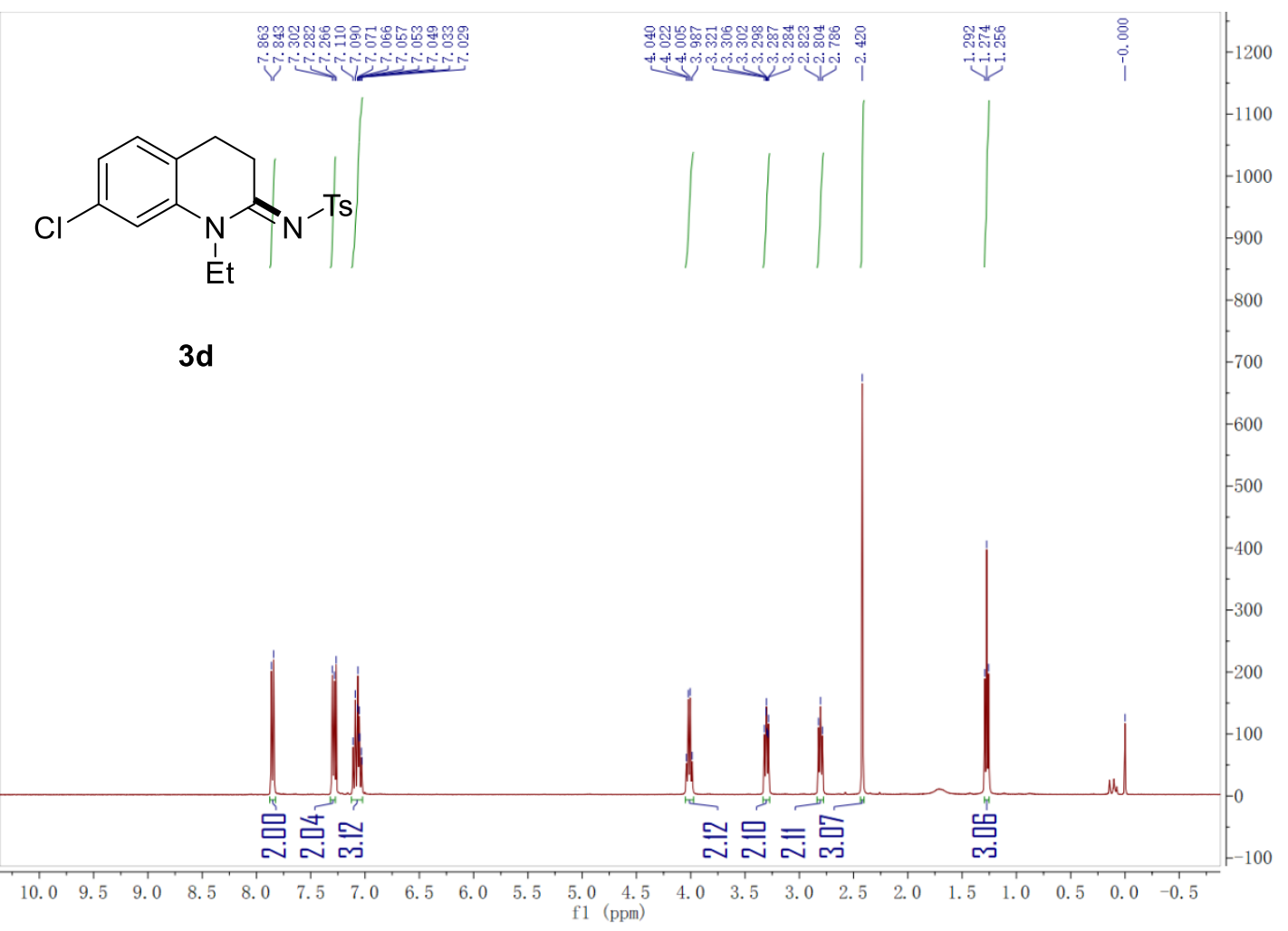

${ }^{1} \mathrm{H}$ NMR spectra (400 MHz, $\mathrm{CDCl}_{3}$ ) of $\mathbf{3 d}$

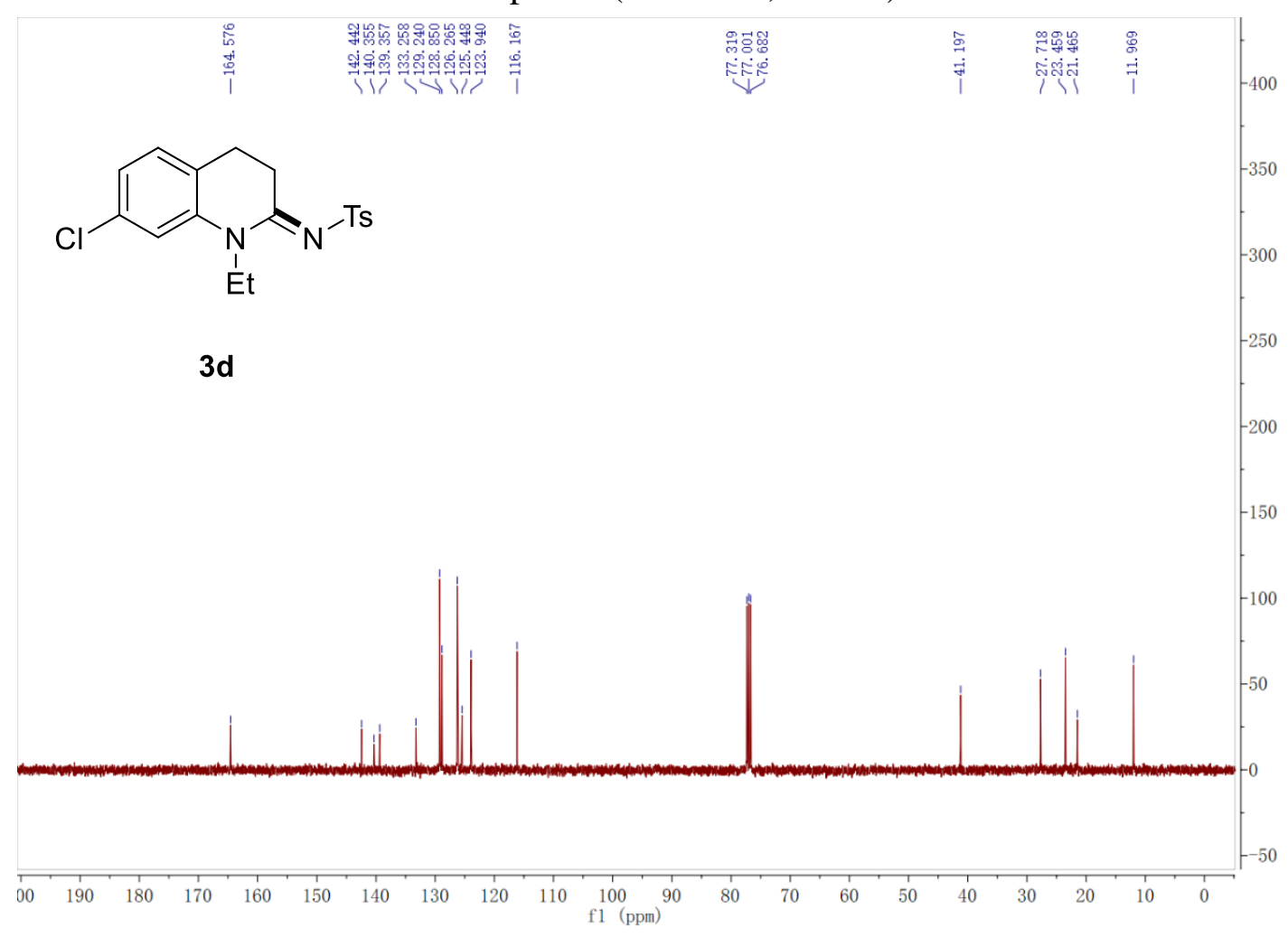

${ }^{13} \mathrm{C}$ NMR spectra $\left(101 \mathrm{MHz}, \mathrm{CDCl}_{3}\right)$ of $\mathbf{3 d}$ 


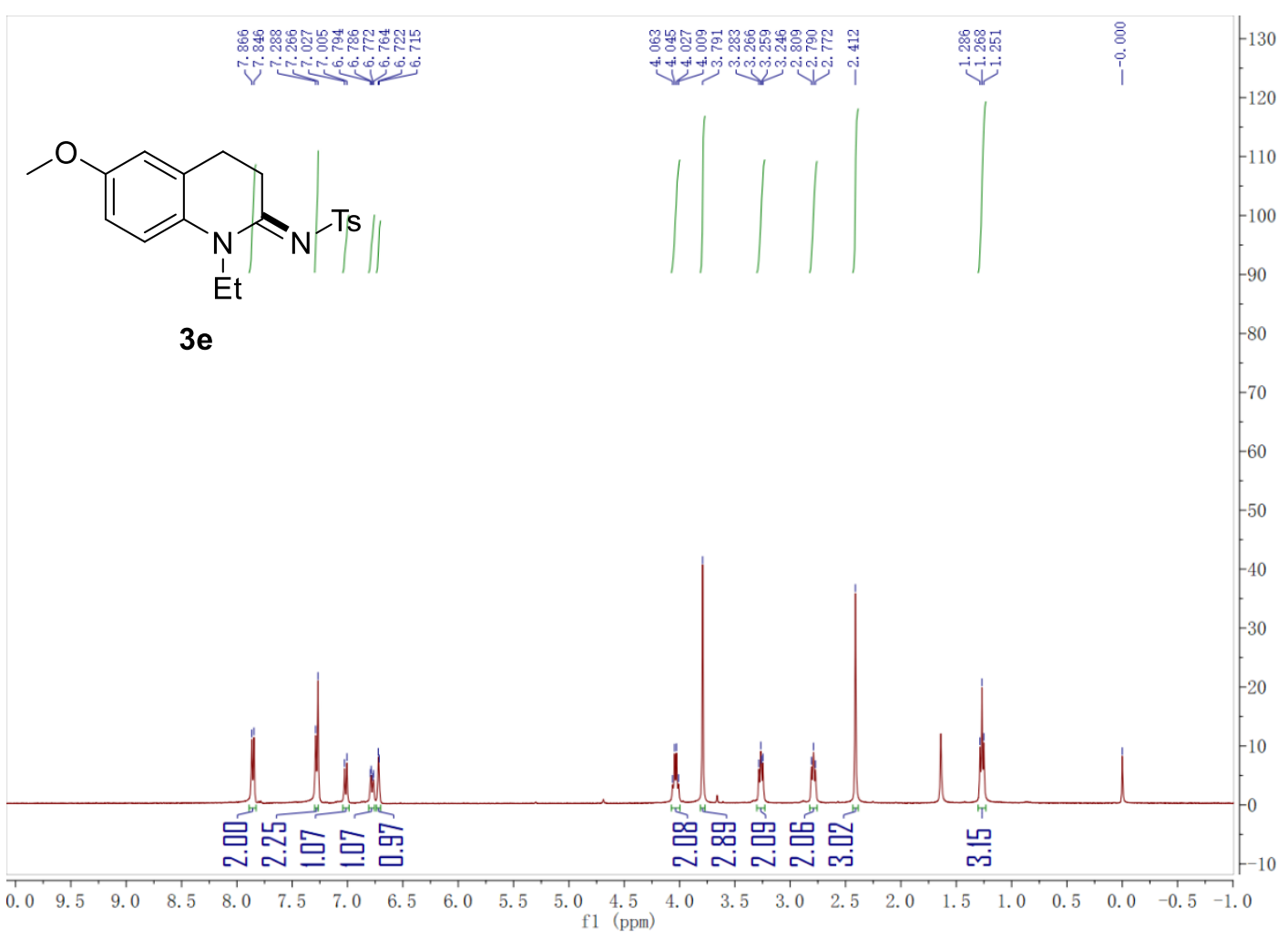

${ }^{1} \mathrm{H}$ NMR spectra $\left(400 \mathrm{MHz}, \mathrm{CDCl}_{3}\right)$ of $\mathbf{3 e}$

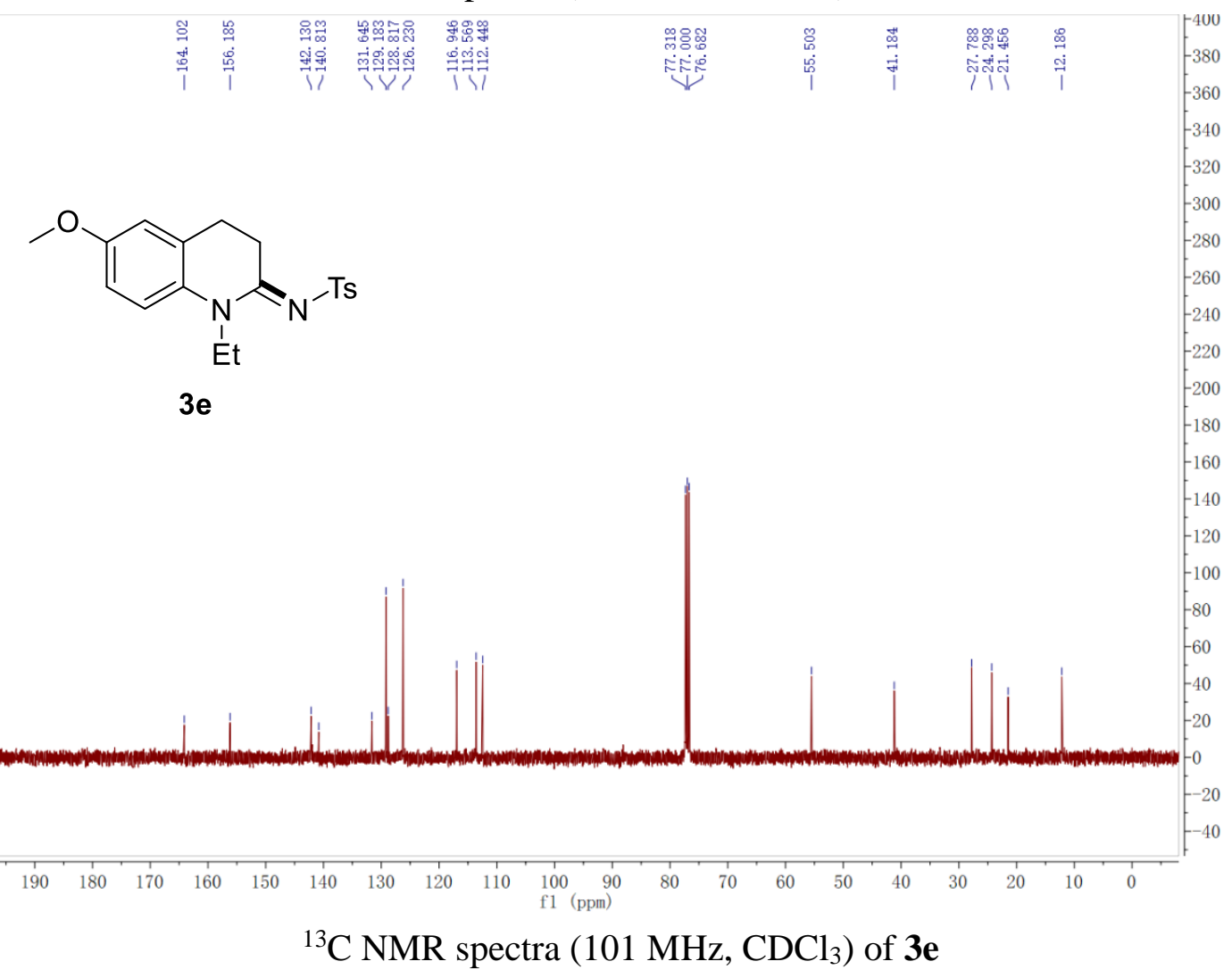




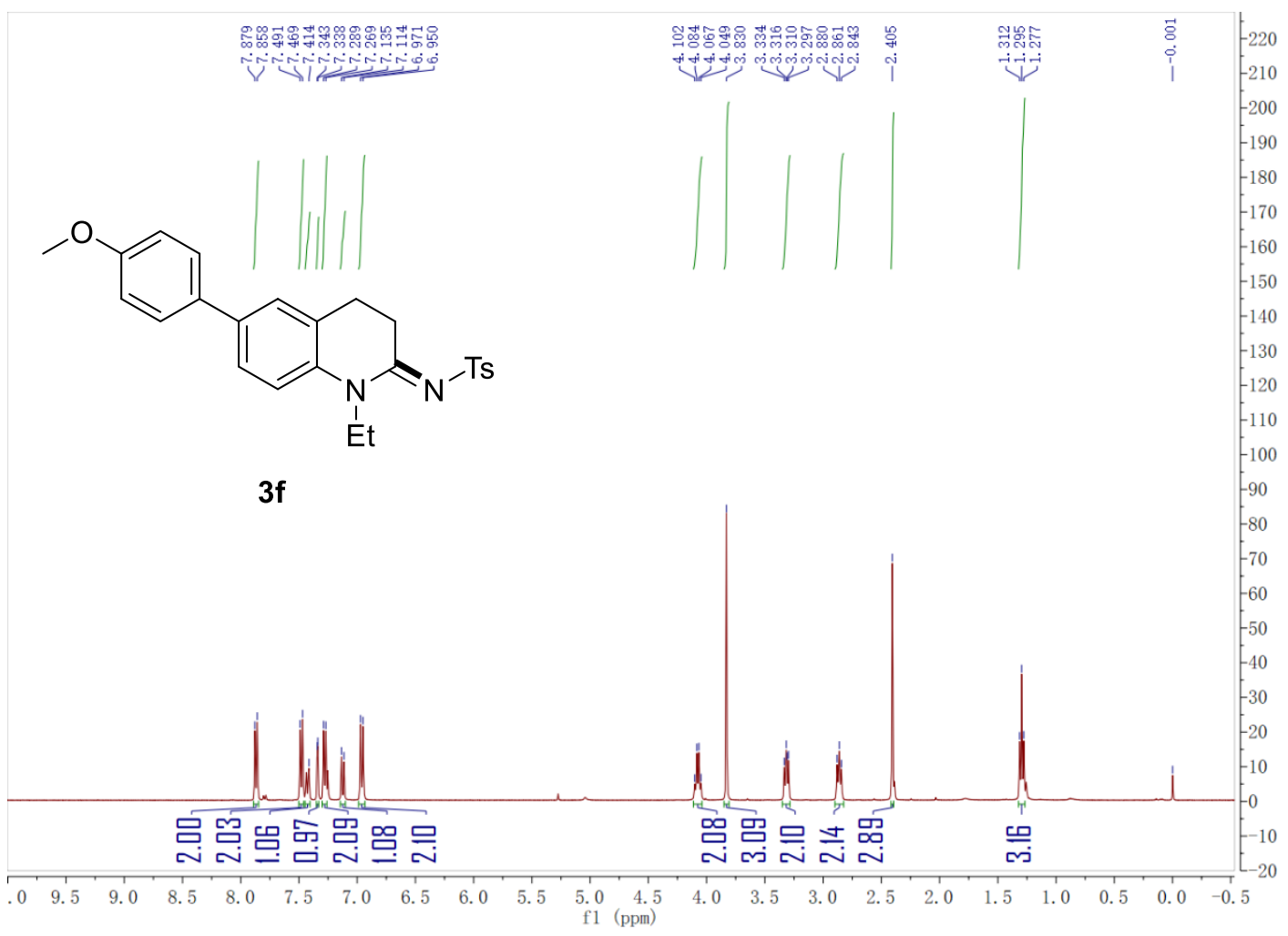

${ }^{1} \mathrm{H}$ NMR spectra $\left(400 \mathrm{MHz}, \mathrm{CDCl}_{3}\right)$ of $\mathbf{3 f}$

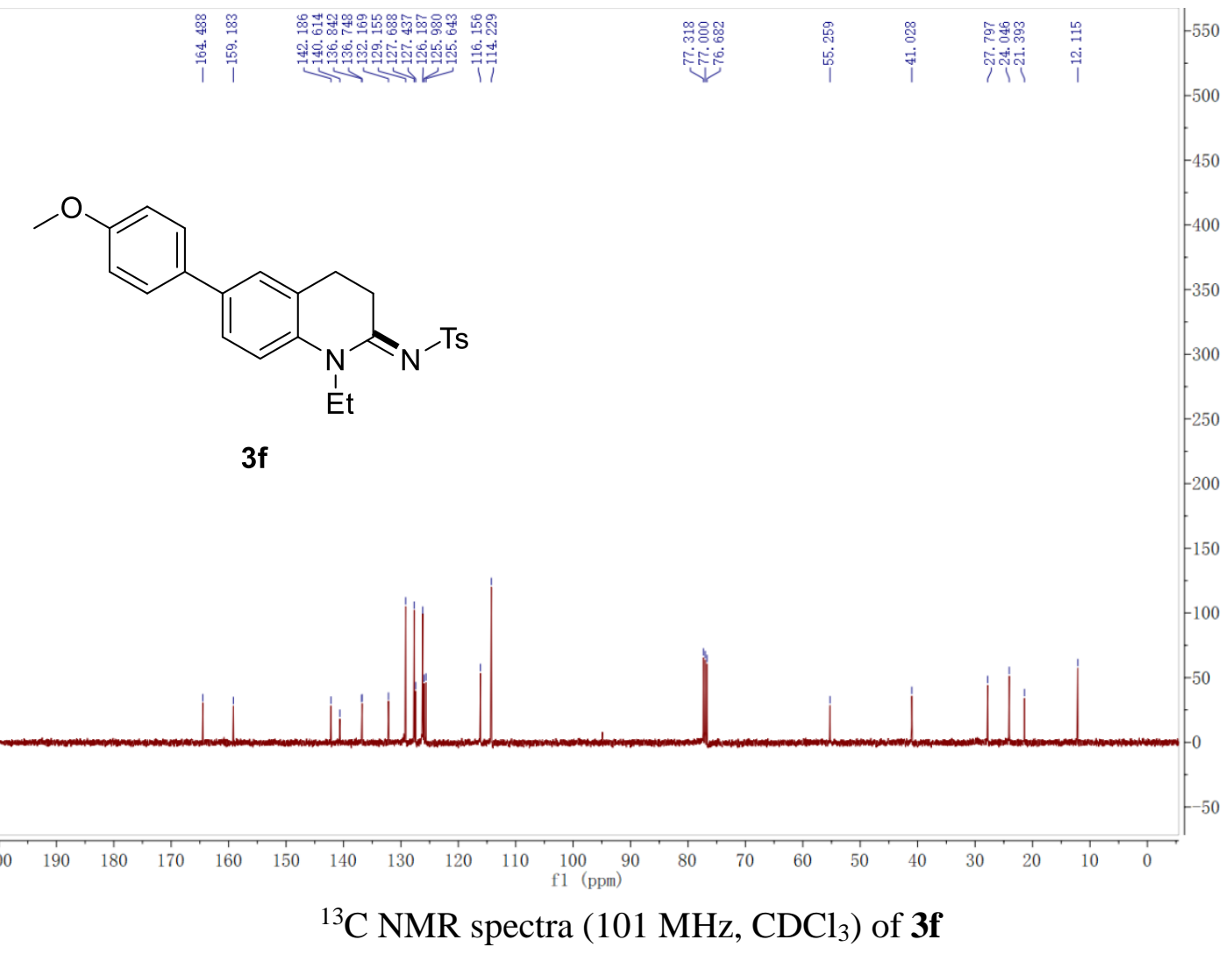




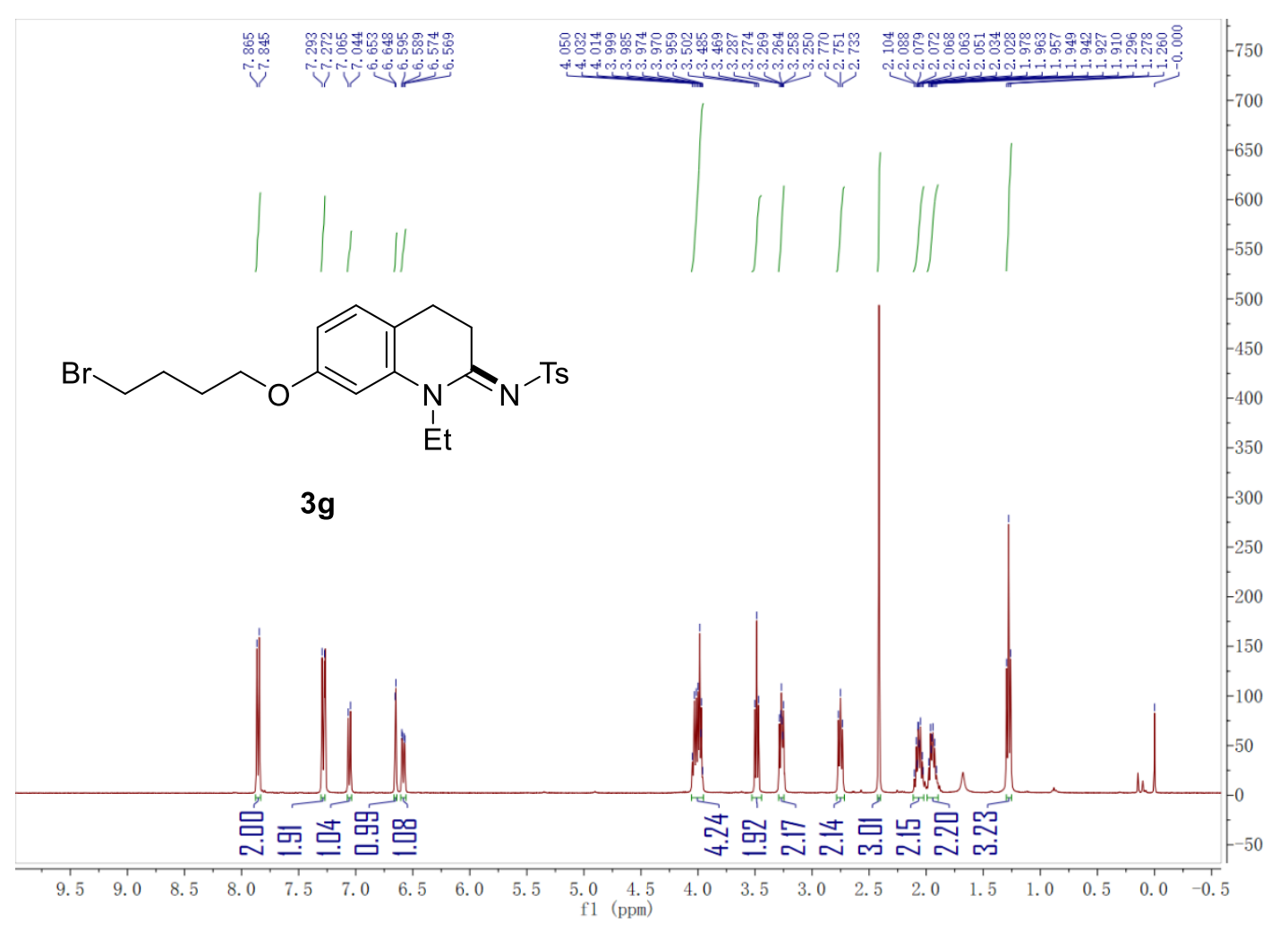

${ }^{1} \mathrm{H}$ NMR spectra (400 MHz, $\mathrm{CDCl}_{3}$ ) of $\mathbf{3 g}$

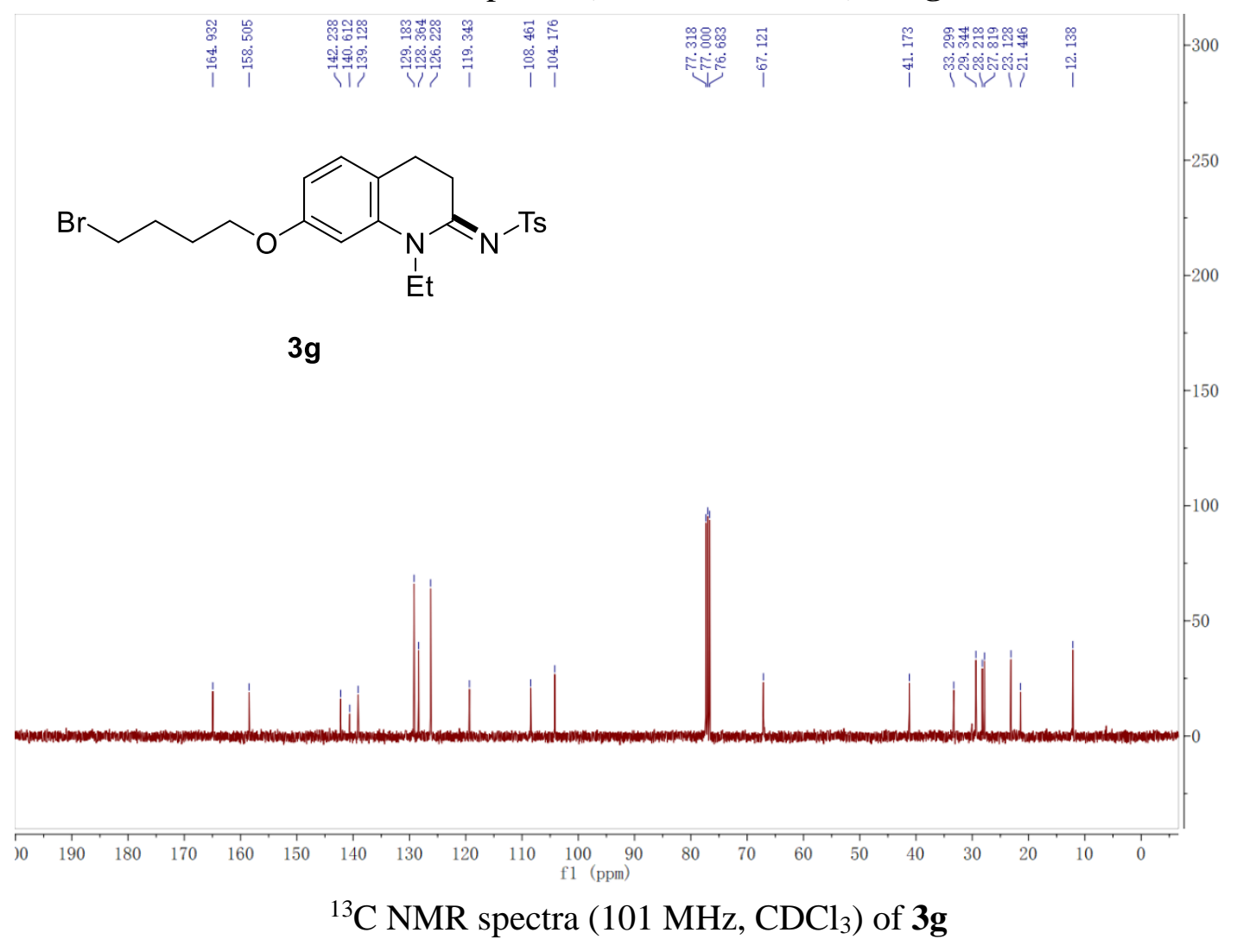




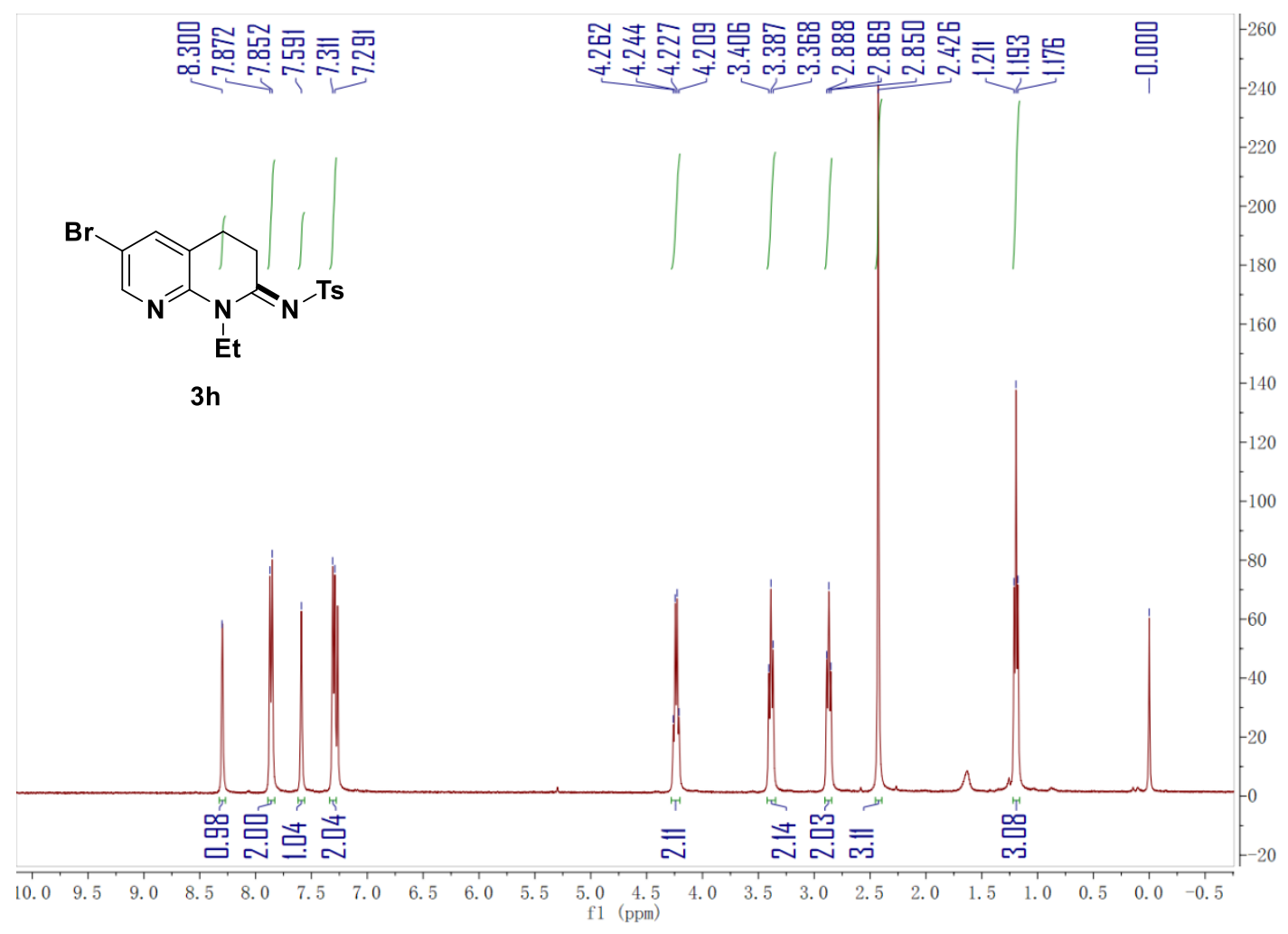

${ }^{1} \mathrm{H}$ NMR spectra (400 MHz, $\mathrm{CDCl}_{3}$ ) of $\mathbf{3 h}$

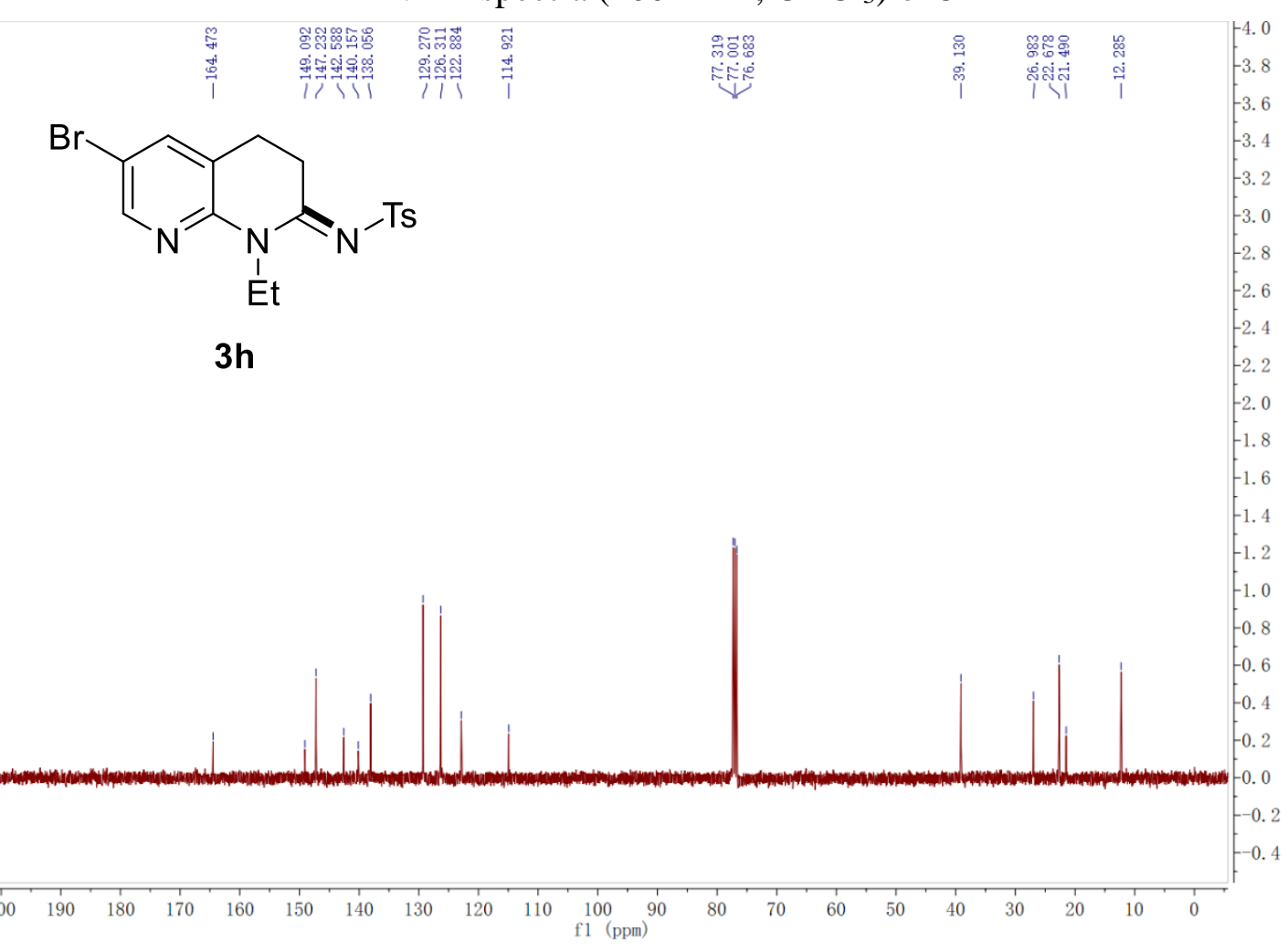

${ }^{13} \mathrm{C}$ NMR spectra $\left(101 \mathrm{MHz}, \mathrm{CDCl}_{3}\right)$ of $\mathbf{3 h}$ 


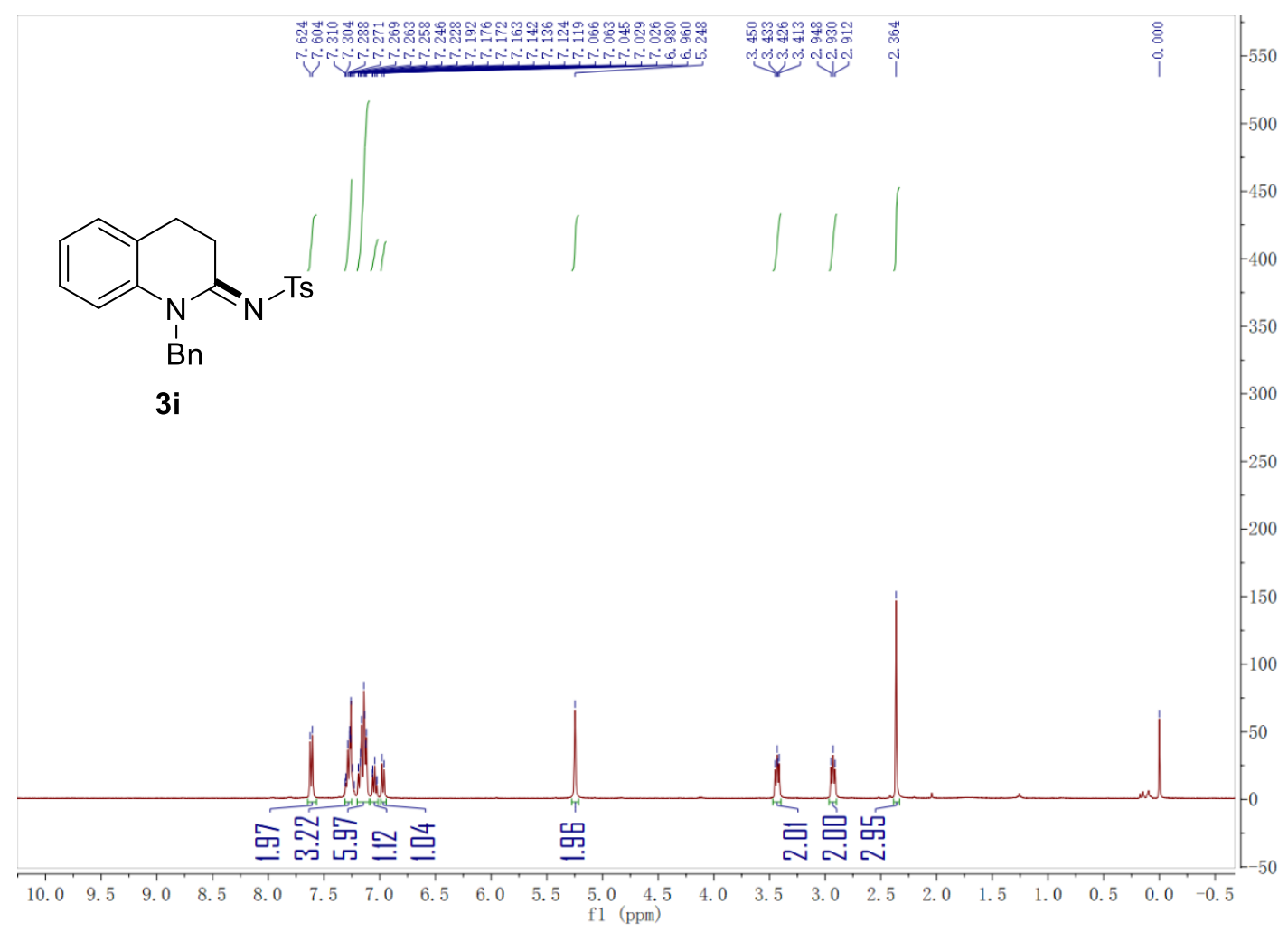

${ }^{1} \mathrm{H}$ NMR spectra $\left(400 \mathrm{MHz}, \mathrm{CDCl}_{3}\right)$ of $\mathbf{3 i}$

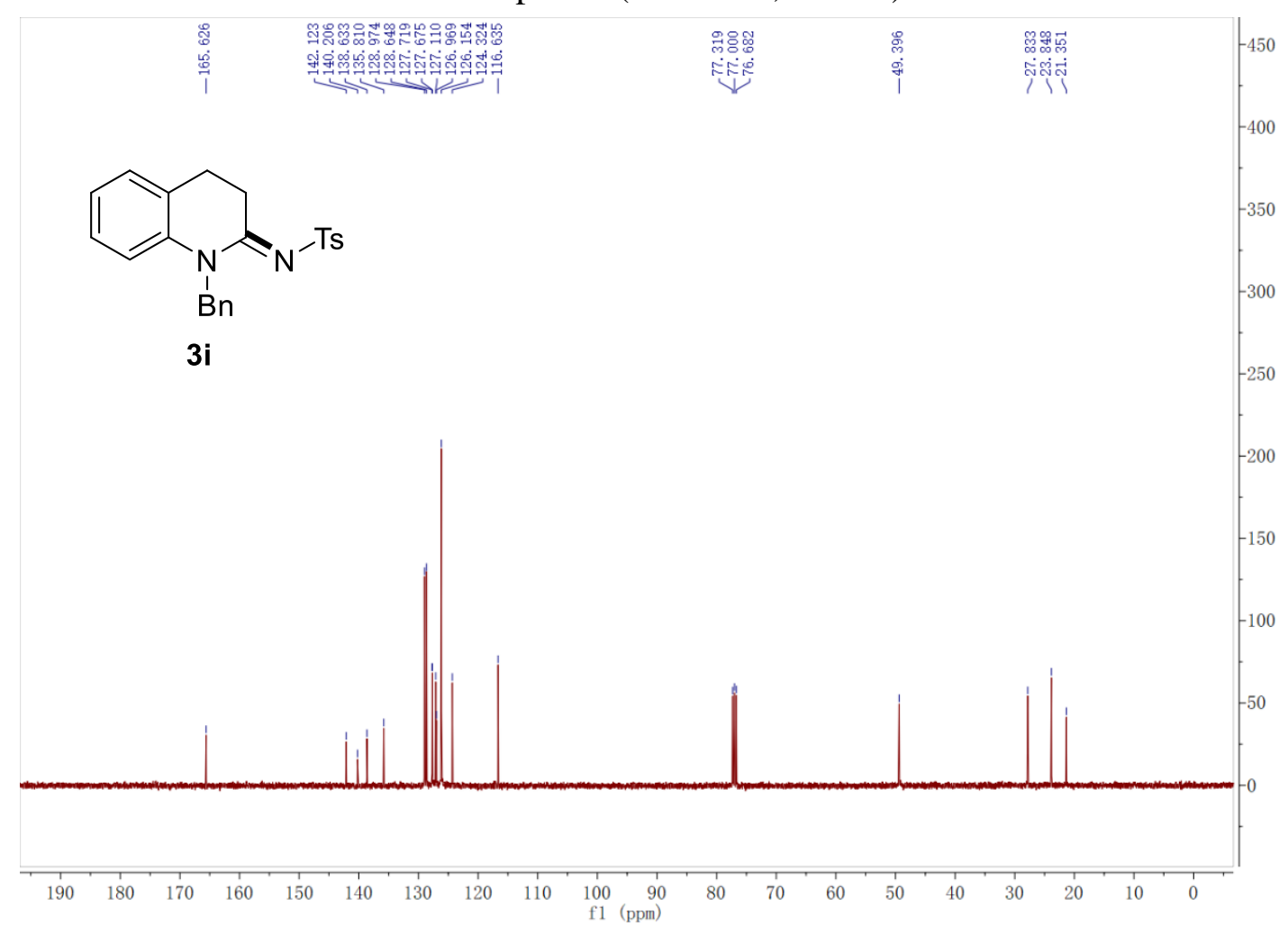

${ }^{13} \mathrm{C}$ NMR spectra $\left(101 \mathrm{MHz}, \mathrm{CDCl}_{3}\right)$ of $\mathbf{3 i}$ 


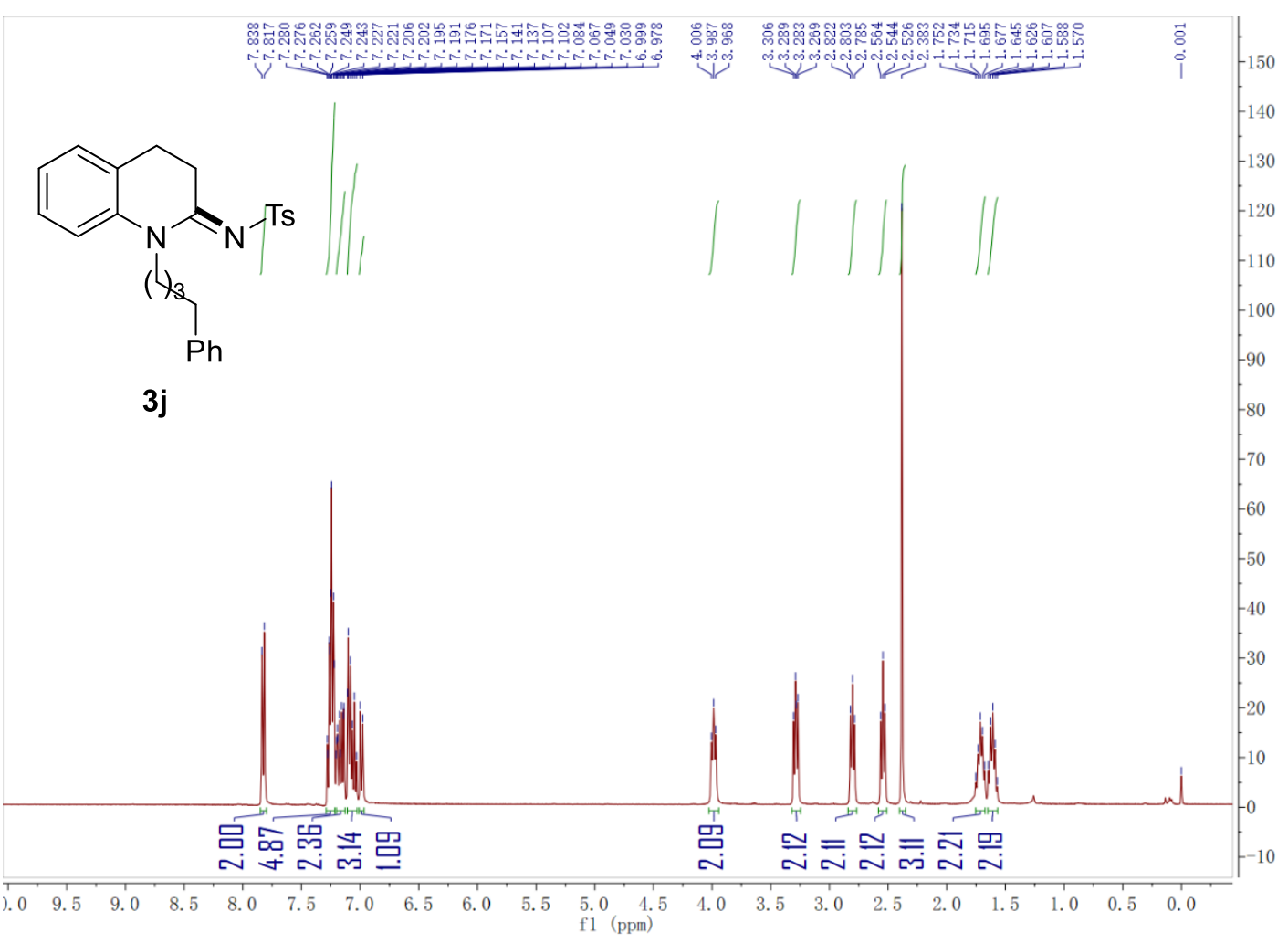

${ }^{1} \mathrm{H}$ NMR spectra (400 MHz, $\mathrm{CDCl}_{3}$ ) of $\mathbf{3 j}$

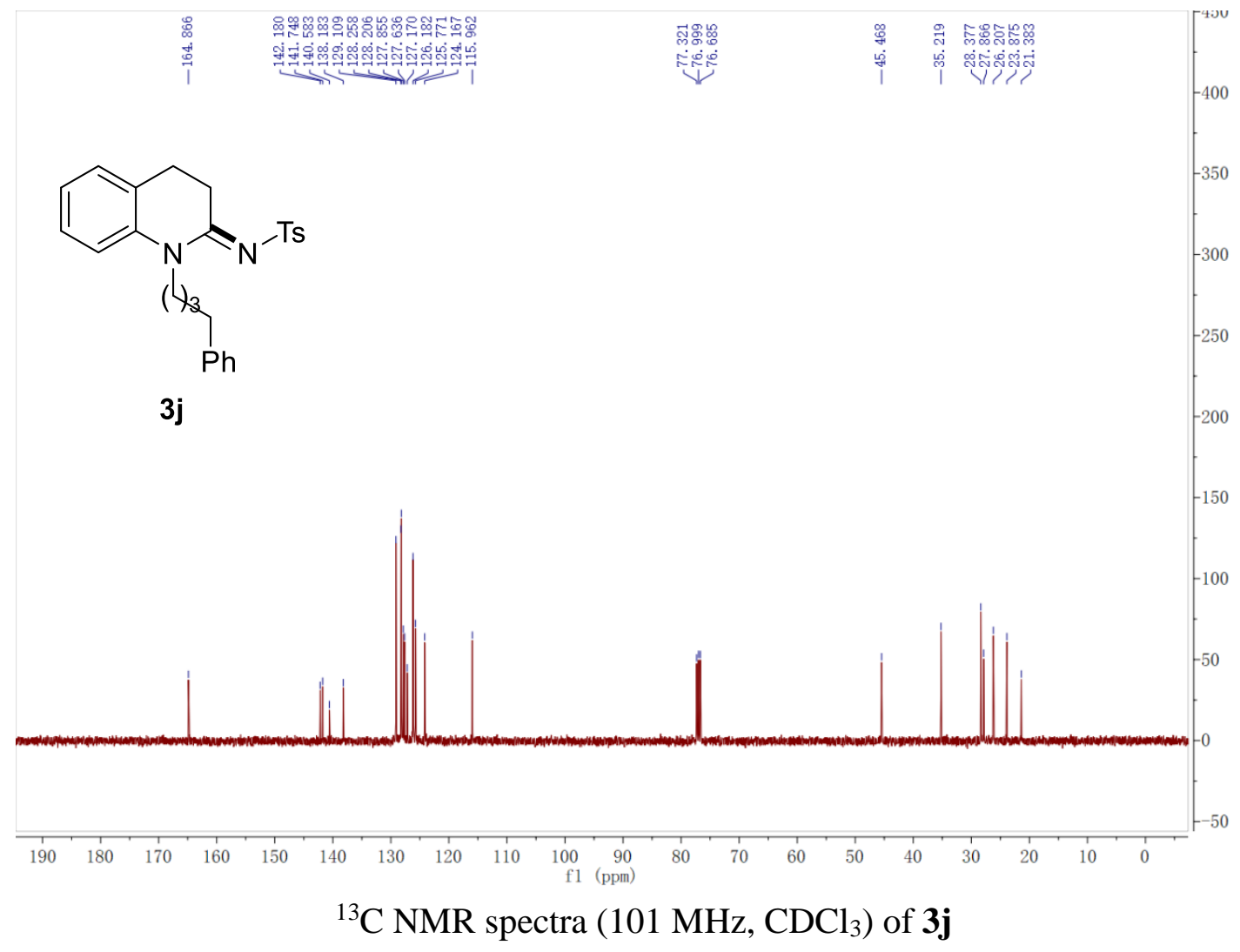




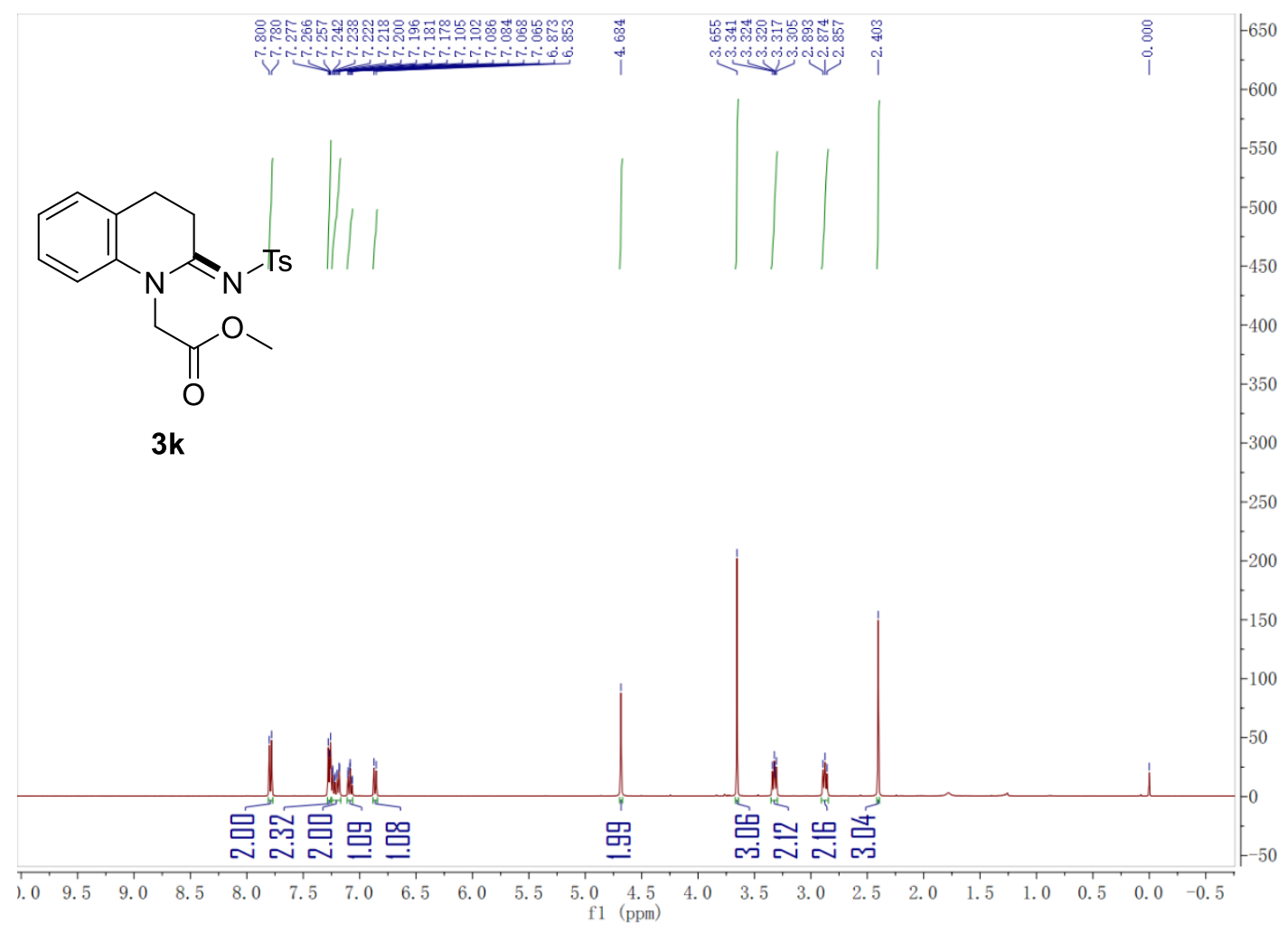

${ }^{1} \mathrm{H}$ NMR spectra $\left(400 \mathrm{MHz}, \mathrm{CDCl}_{3}\right)$ of $\mathbf{3 k}$

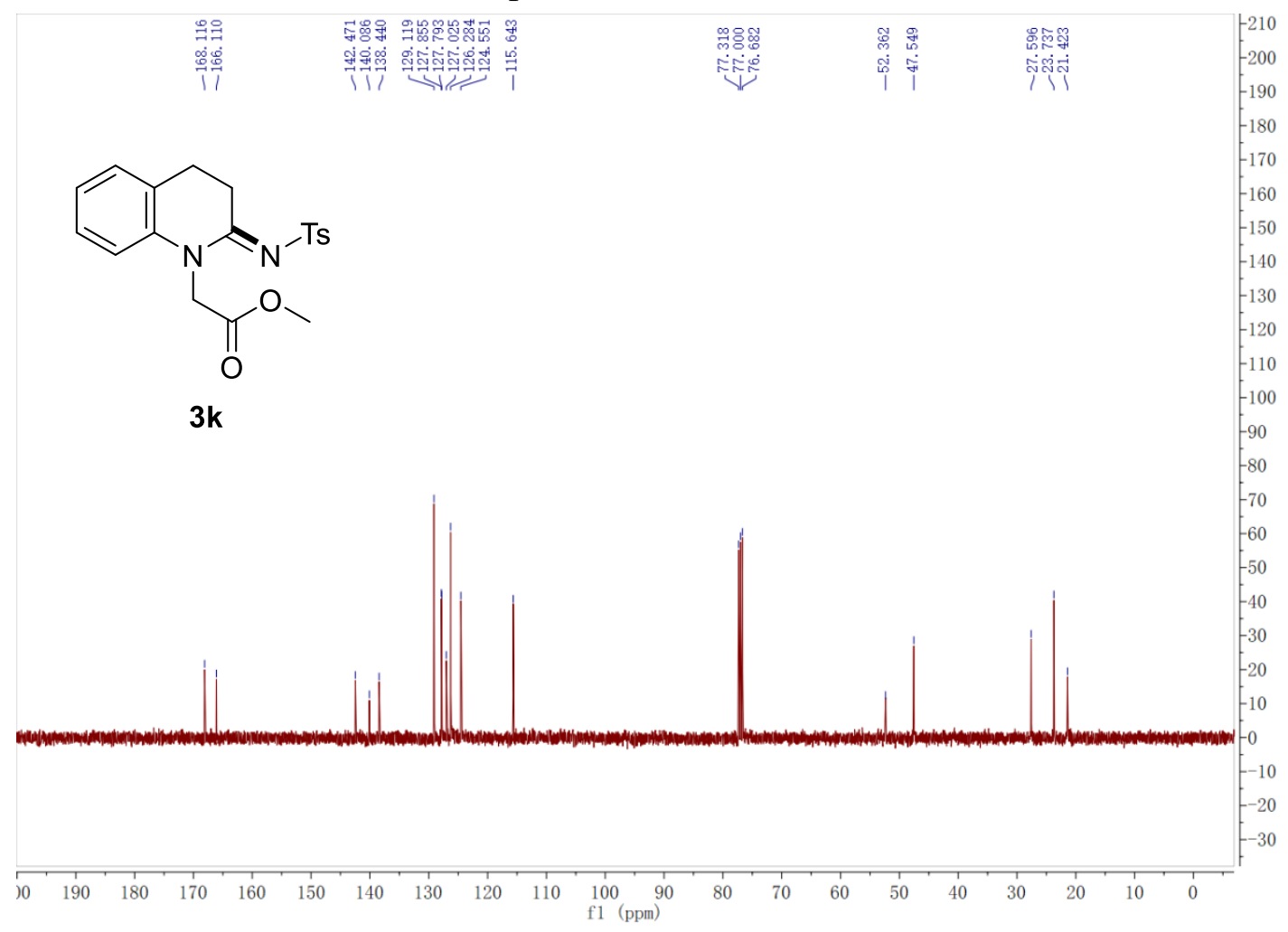

${ }^{13} \mathrm{C}$ NMR spectra $\left(101 \mathrm{MHz}, \mathrm{CDCl}_{3}\right)$ of $\mathbf{3 k}$ 


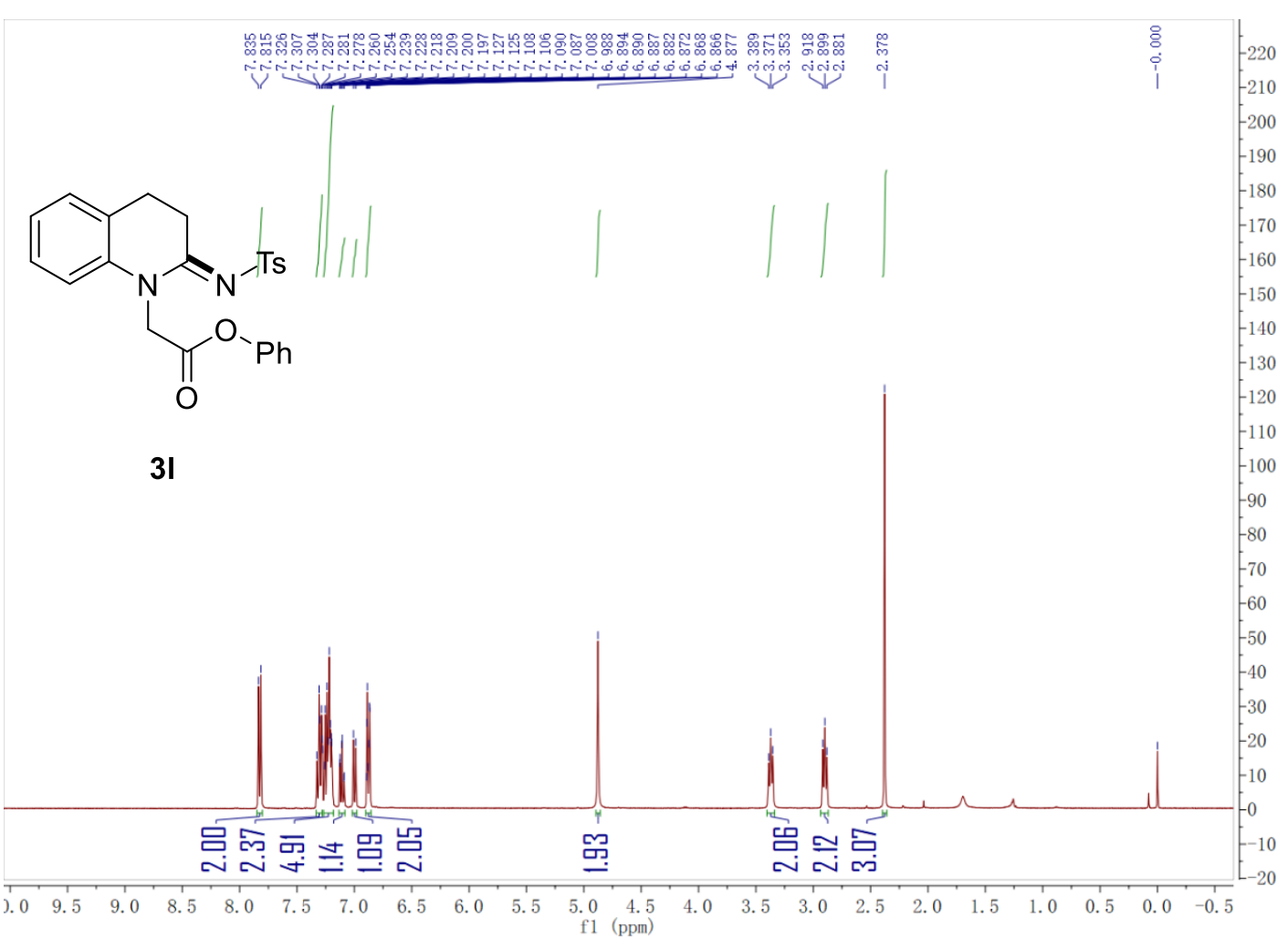

${ }^{1} \mathrm{H}$ NMR spectra $\left(400 \mathrm{MHz}, \mathrm{CDCl}_{3}\right)$ of $\mathbf{3 l}$

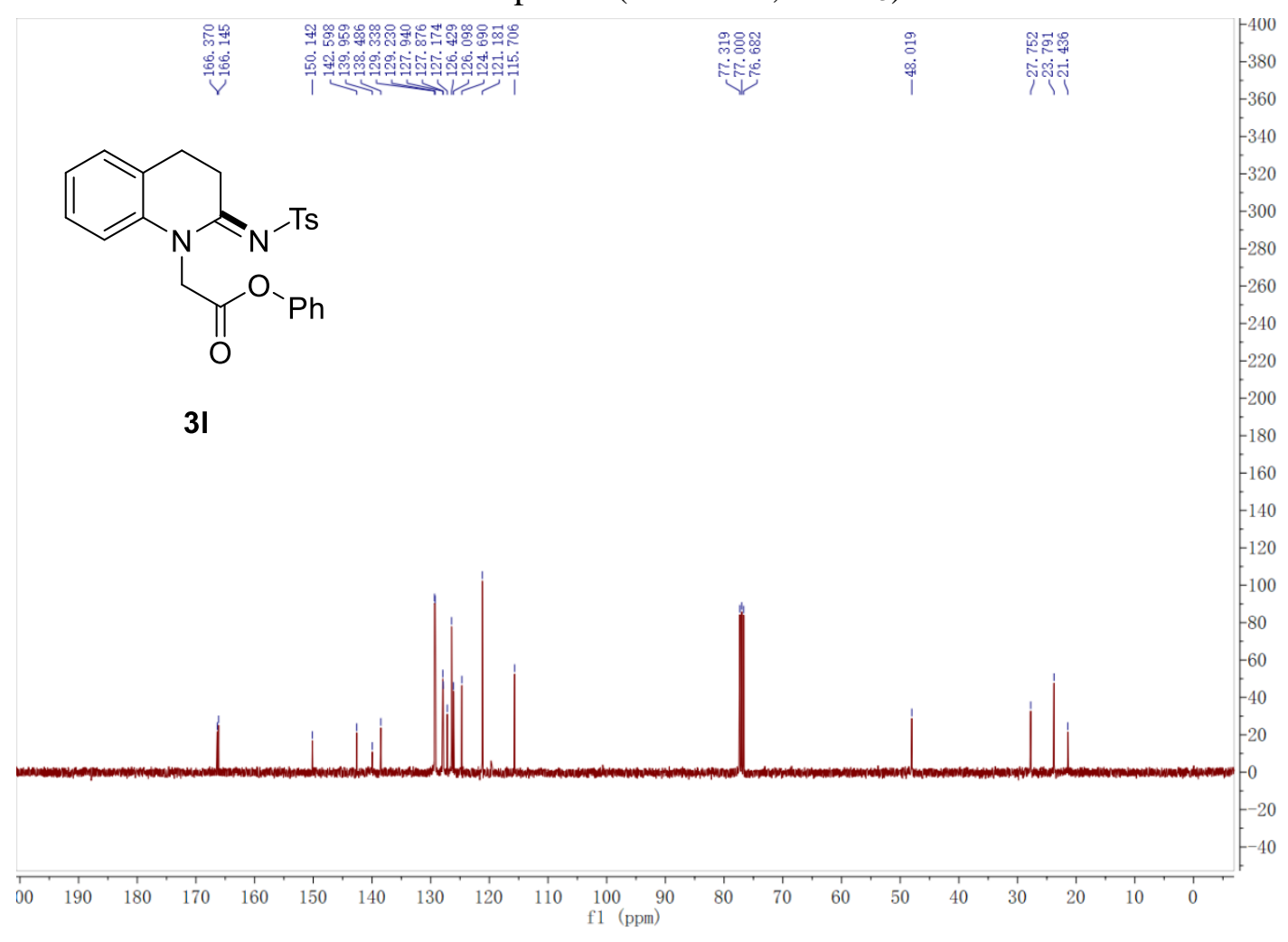

${ }^{13} \mathrm{C}$ NMR spectra $\left(101 \mathrm{MHz}, \mathrm{CDCl}_{3}\right)$ of $\mathbf{3 l}$ 


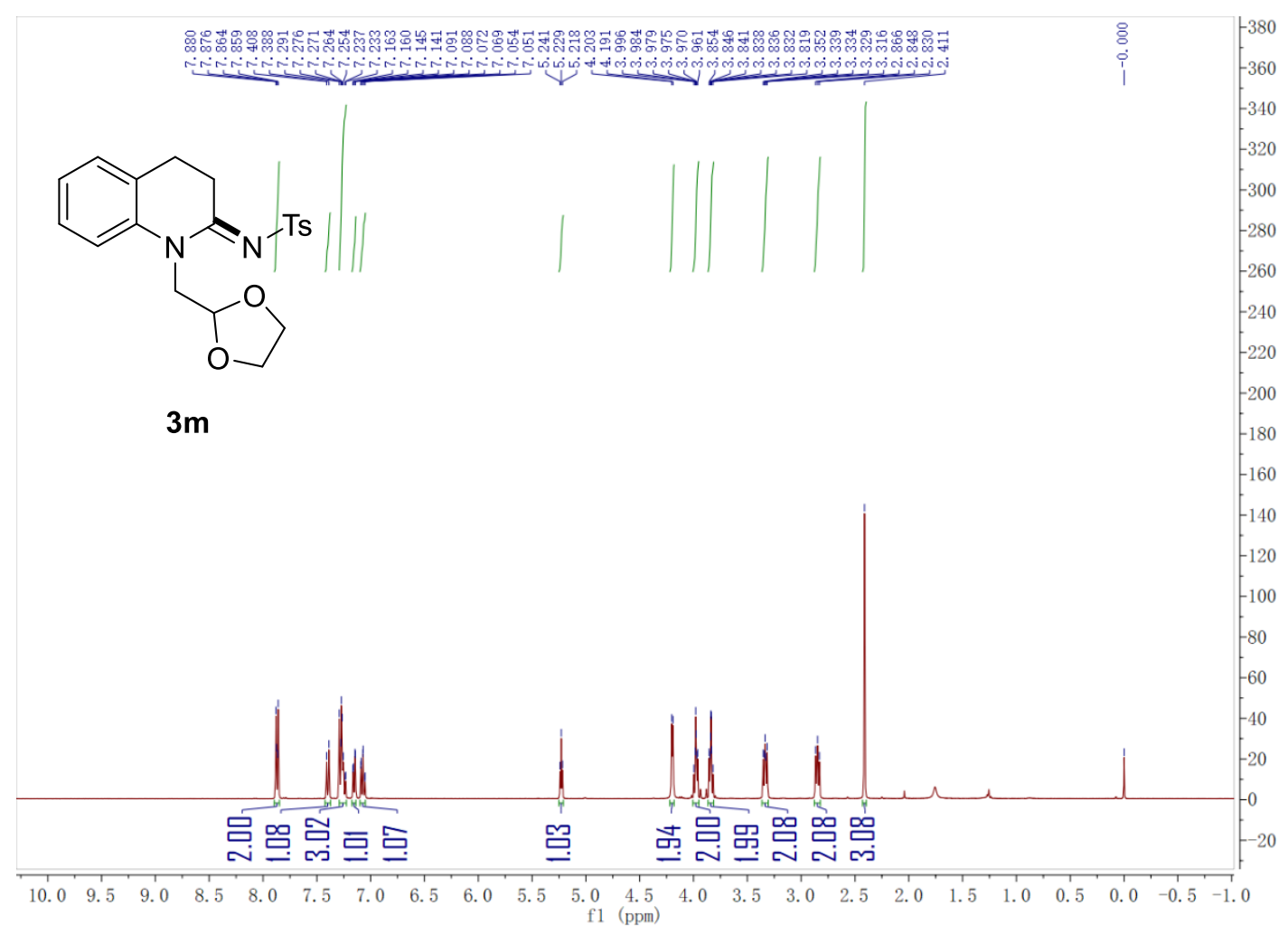

${ }^{1} \mathrm{H}$ NMR spectra $\left(400 \mathrm{MHz}, \mathrm{CDCl}_{3}\right)$ of $\mathbf{3 m}$

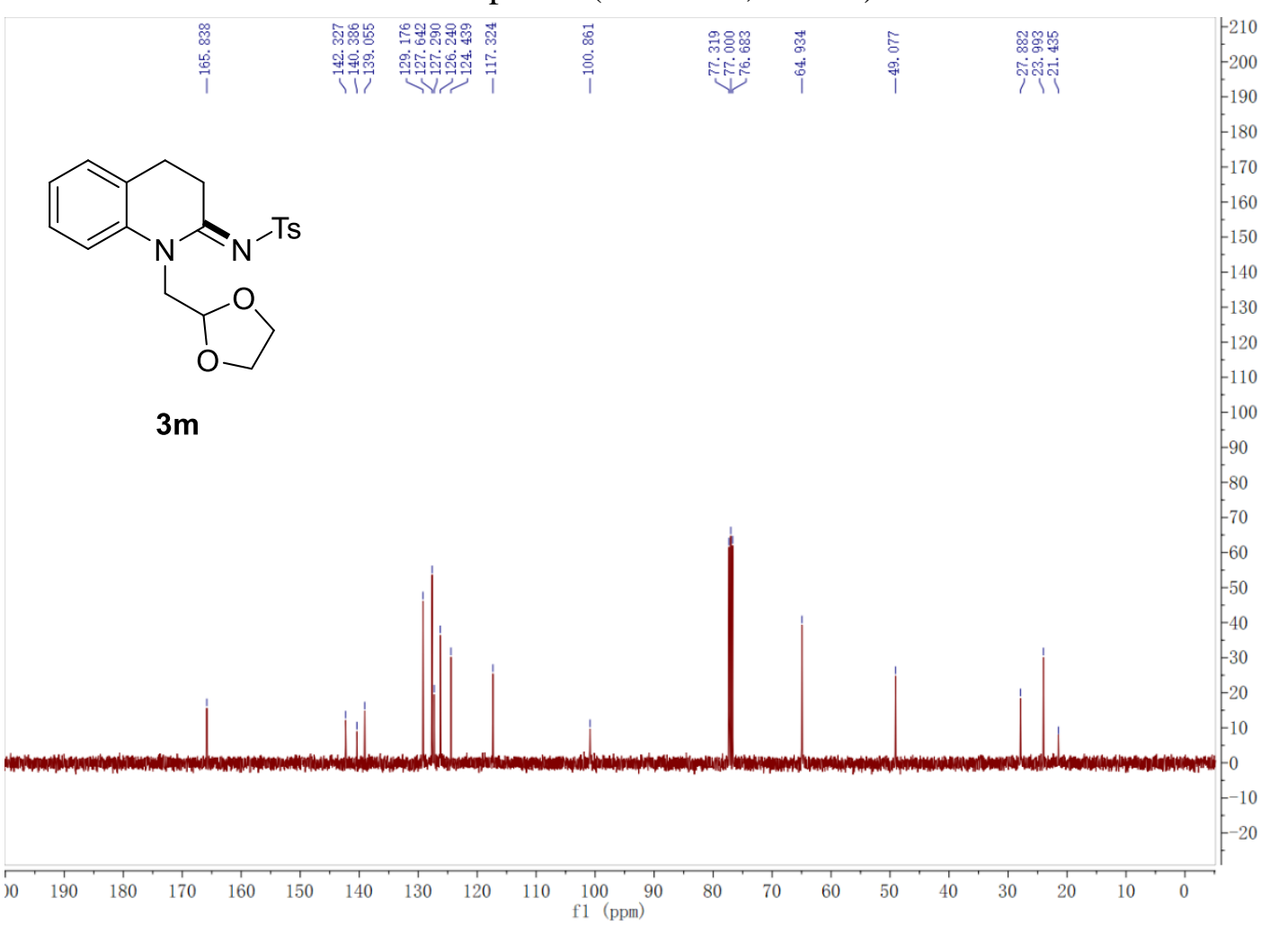

${ }^{13} \mathrm{C}$ NMR spectra $\left(101 \mathrm{MHz}, \mathrm{CDCl}_{3}\right)$ of $\mathbf{3 m}$ 


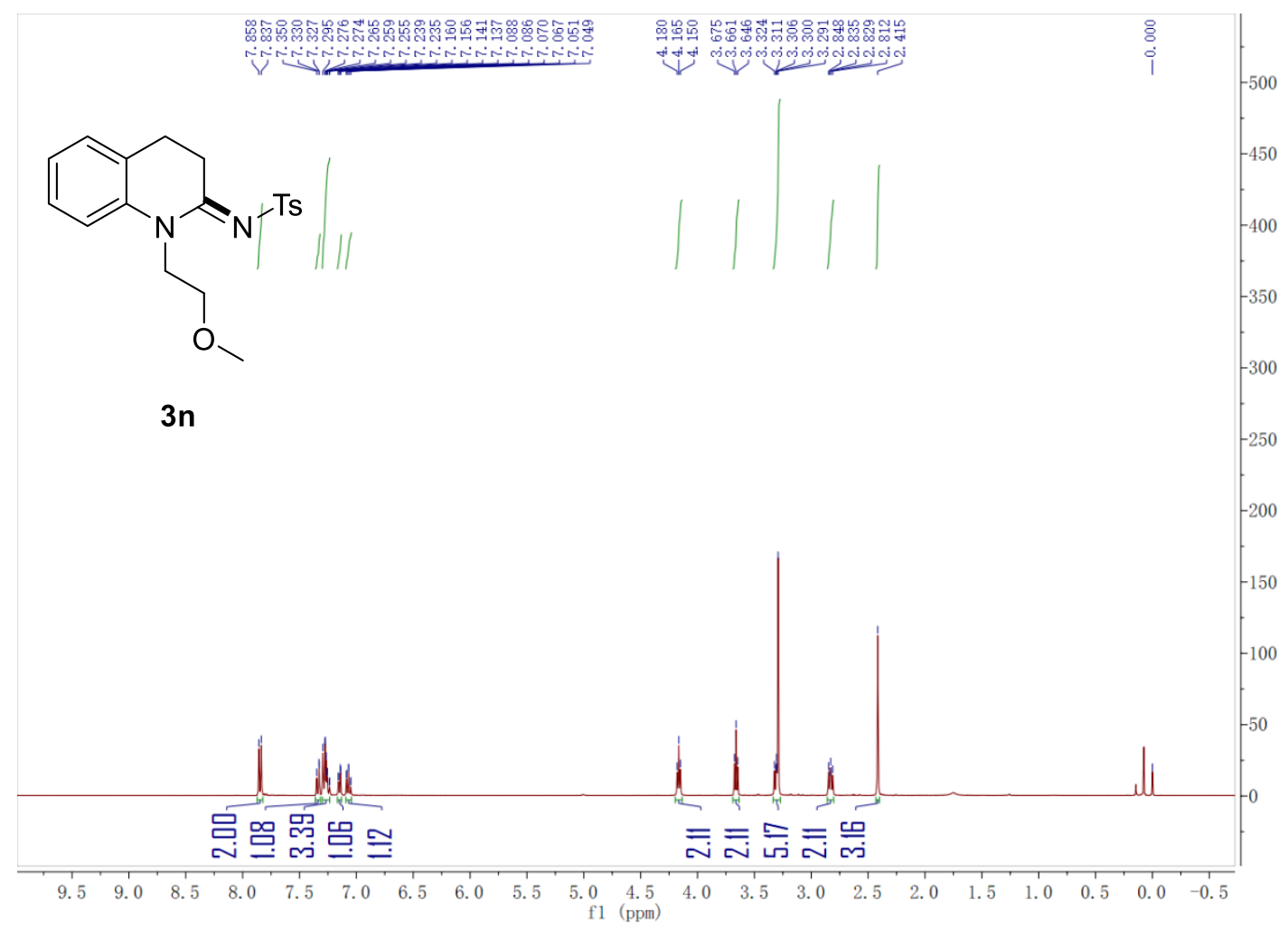

${ }^{1} \mathrm{H}$ NMR spectra (400 MHz, $\mathrm{CDCl}_{3}$ ) of $\mathbf{3 n}$

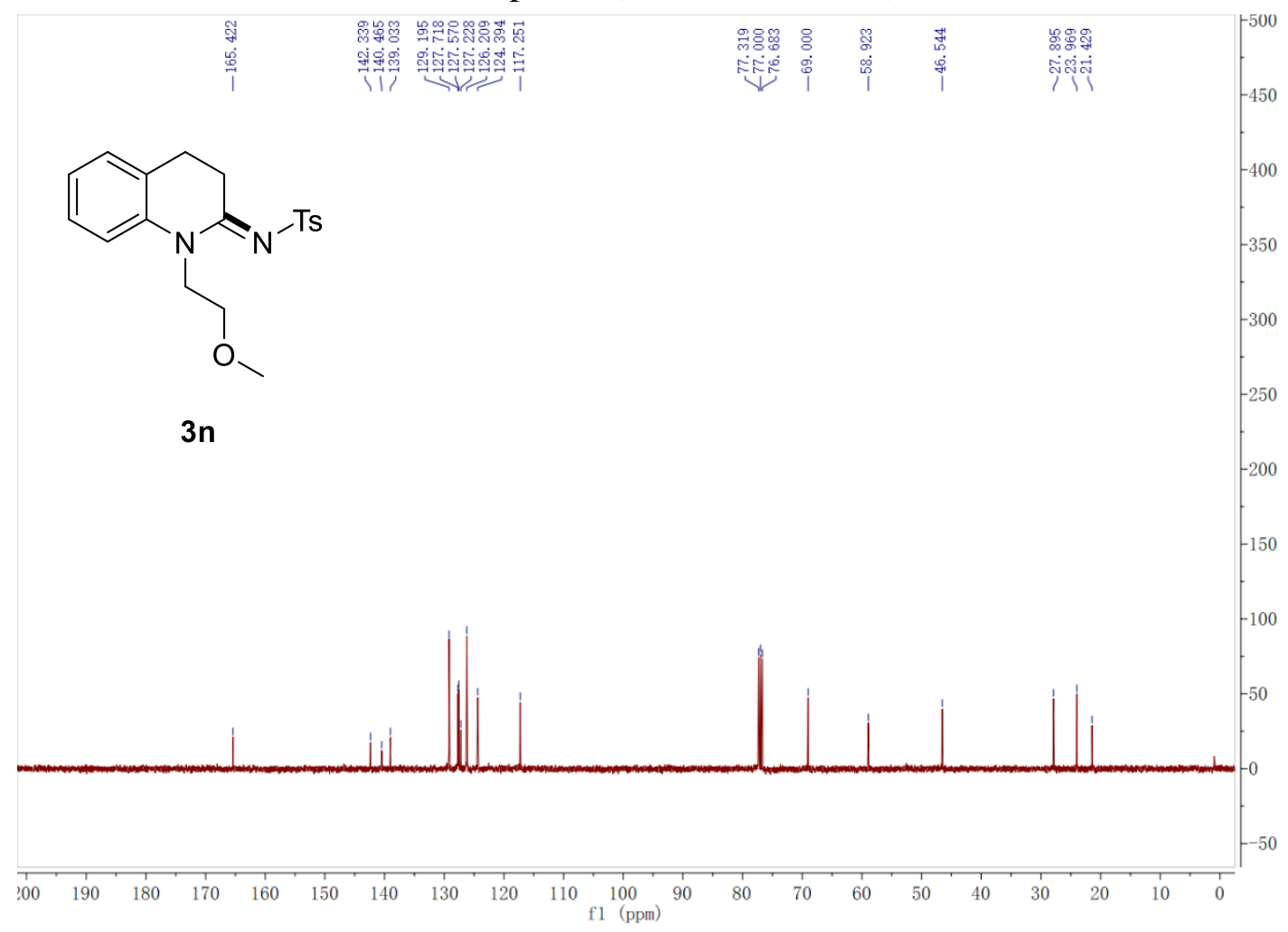

${ }^{13} \mathrm{C}$ NMR spectra $\left(101 \mathrm{MHz}, \mathrm{CDCl}_{3}\right)$ of $\mathbf{3 n}$ 


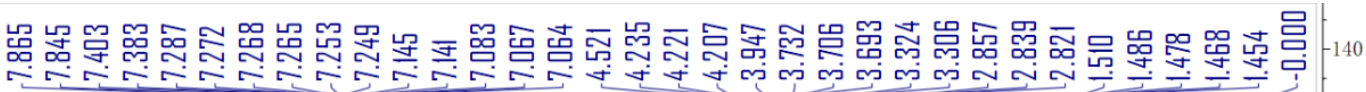<smiles>C#CC1CCc2ccccc2N1CCOC1CCCCO1</smiles>

30

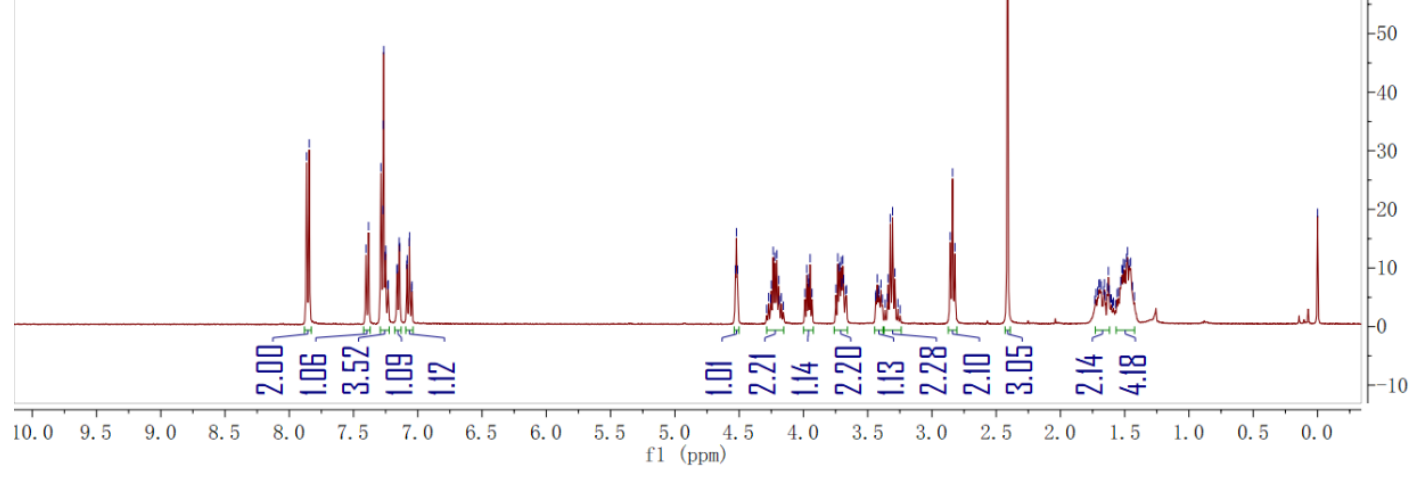

${ }^{1} \mathrm{H}$ NMR spectra (400 MHz, $\mathrm{CDCl}_{3}$ ) of $\mathbf{3 o}$

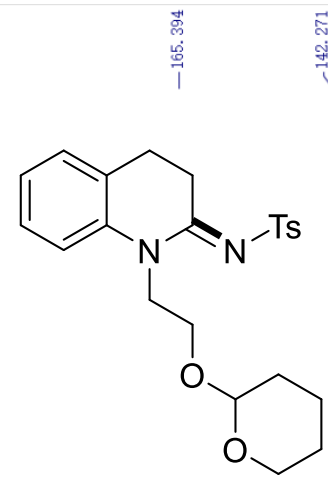

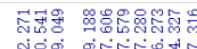

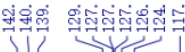

30

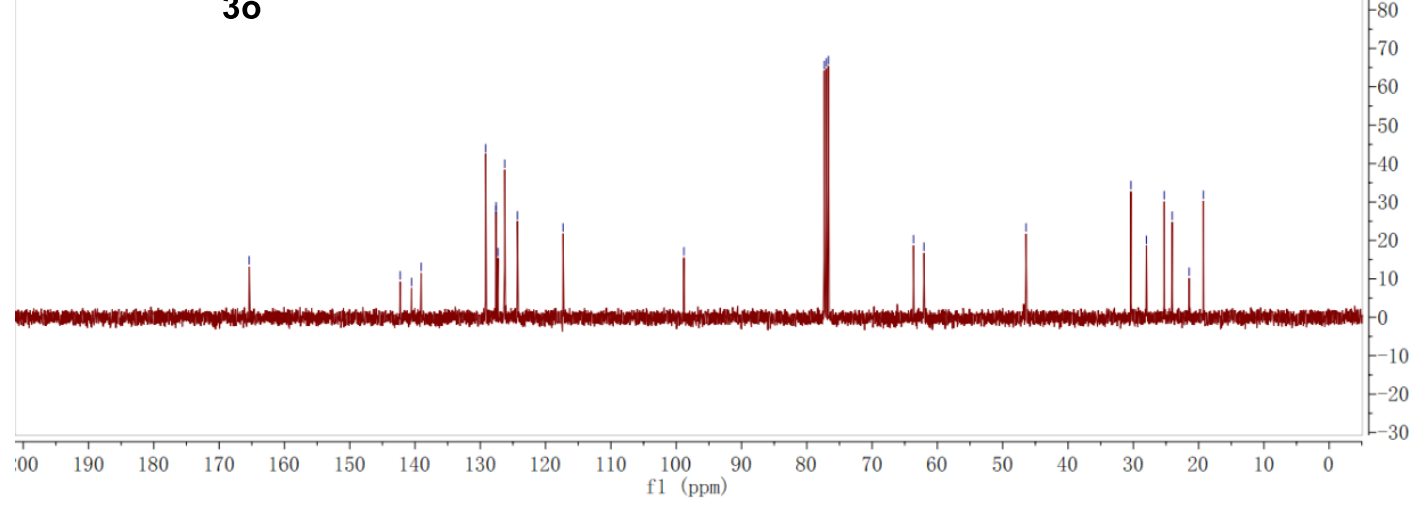

${ }^{13} \mathrm{C}$ NMR spectra $\left(101 \mathrm{MHz}, \mathrm{CDCl}_{3}\right)$ of $\mathbf{3 o}$ 

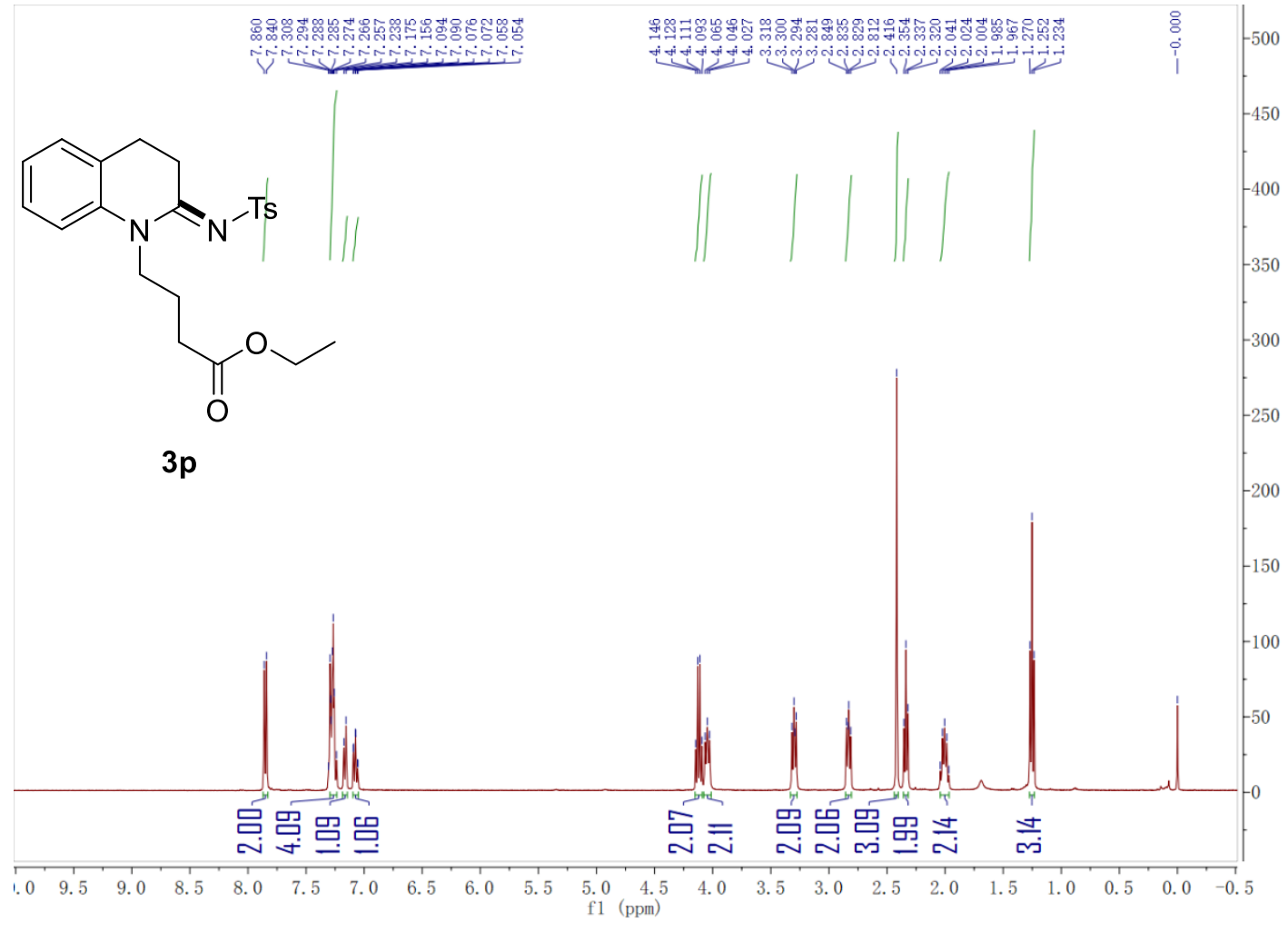

${ }^{1} \mathrm{H}$ NMR spectra (400 MHz, $\mathrm{CDCl}_{3}$ ) of $\mathbf{3 p}$

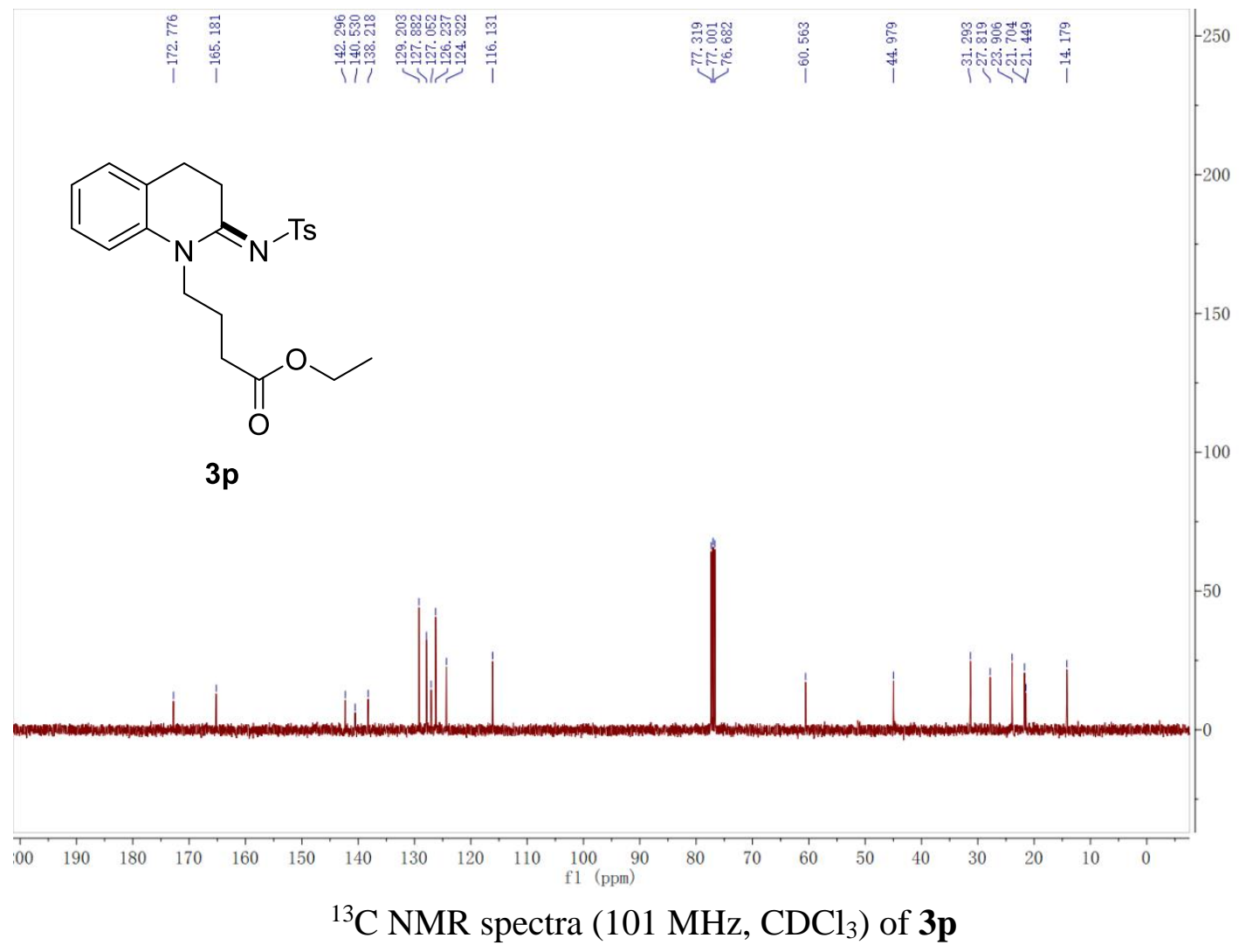




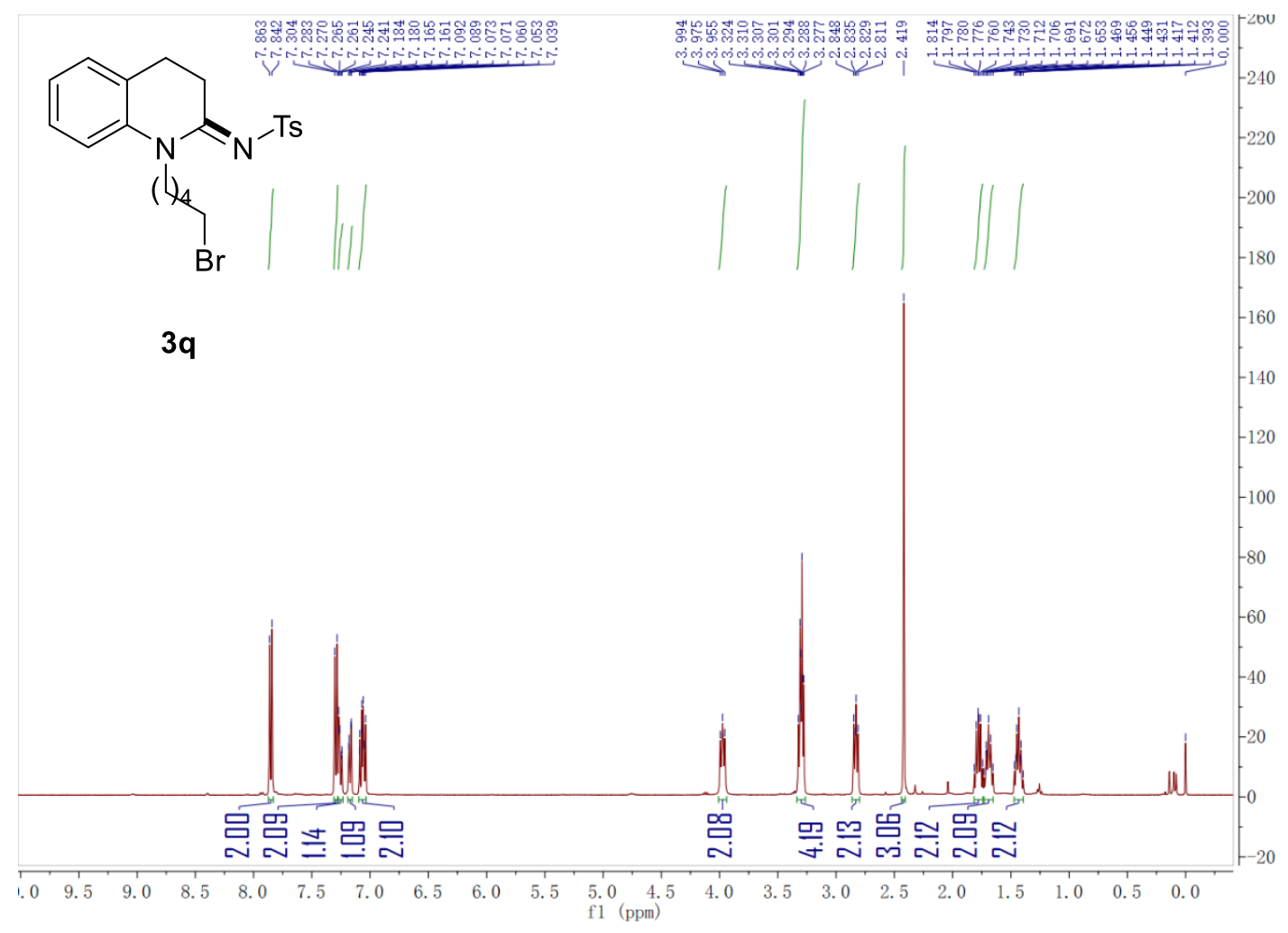

${ }^{1} \mathrm{H}$ NMR spectra $\left(400 \mathrm{MHz}, \mathrm{CDCl}_{3}\right.$ ) of $\mathbf{3 q}$

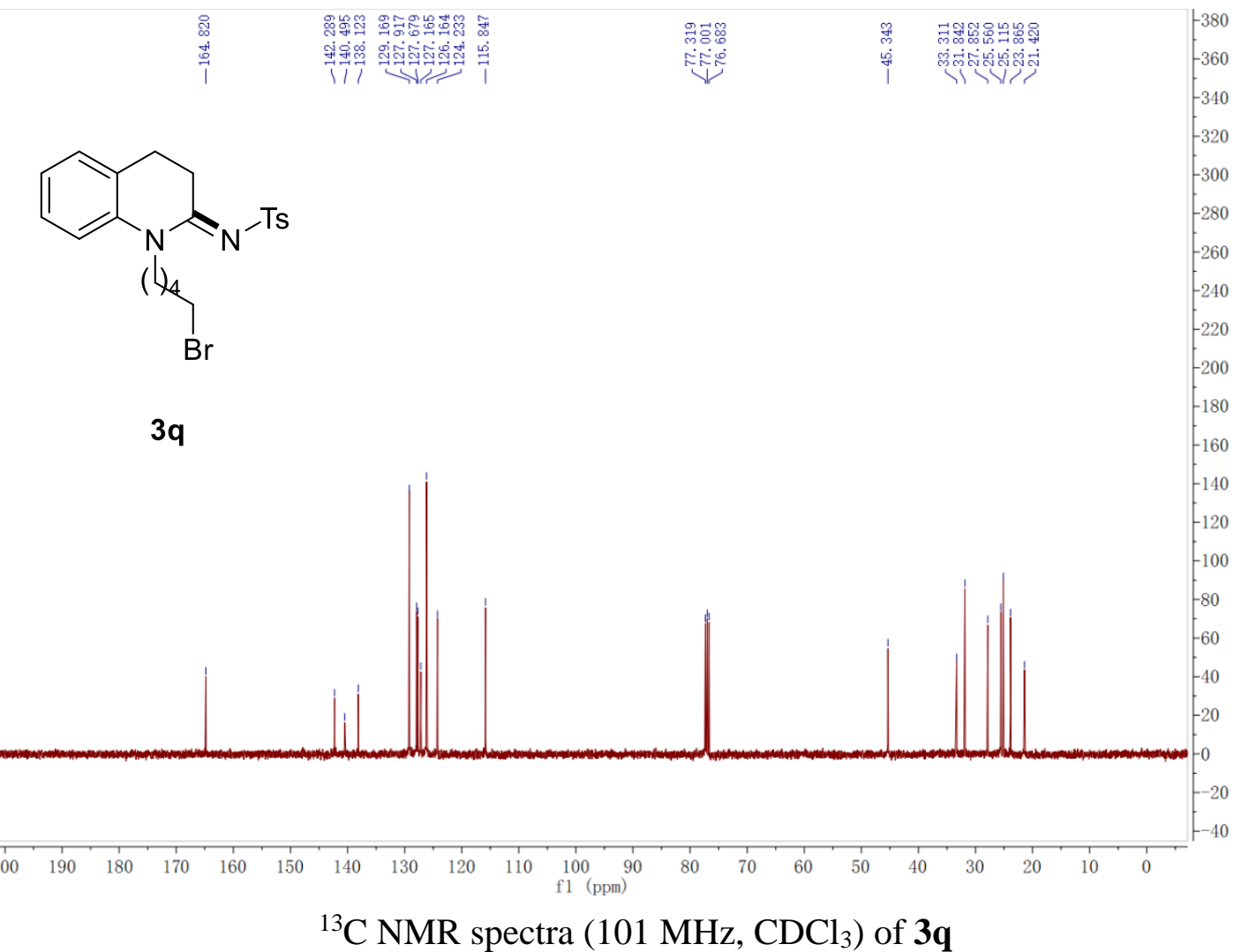




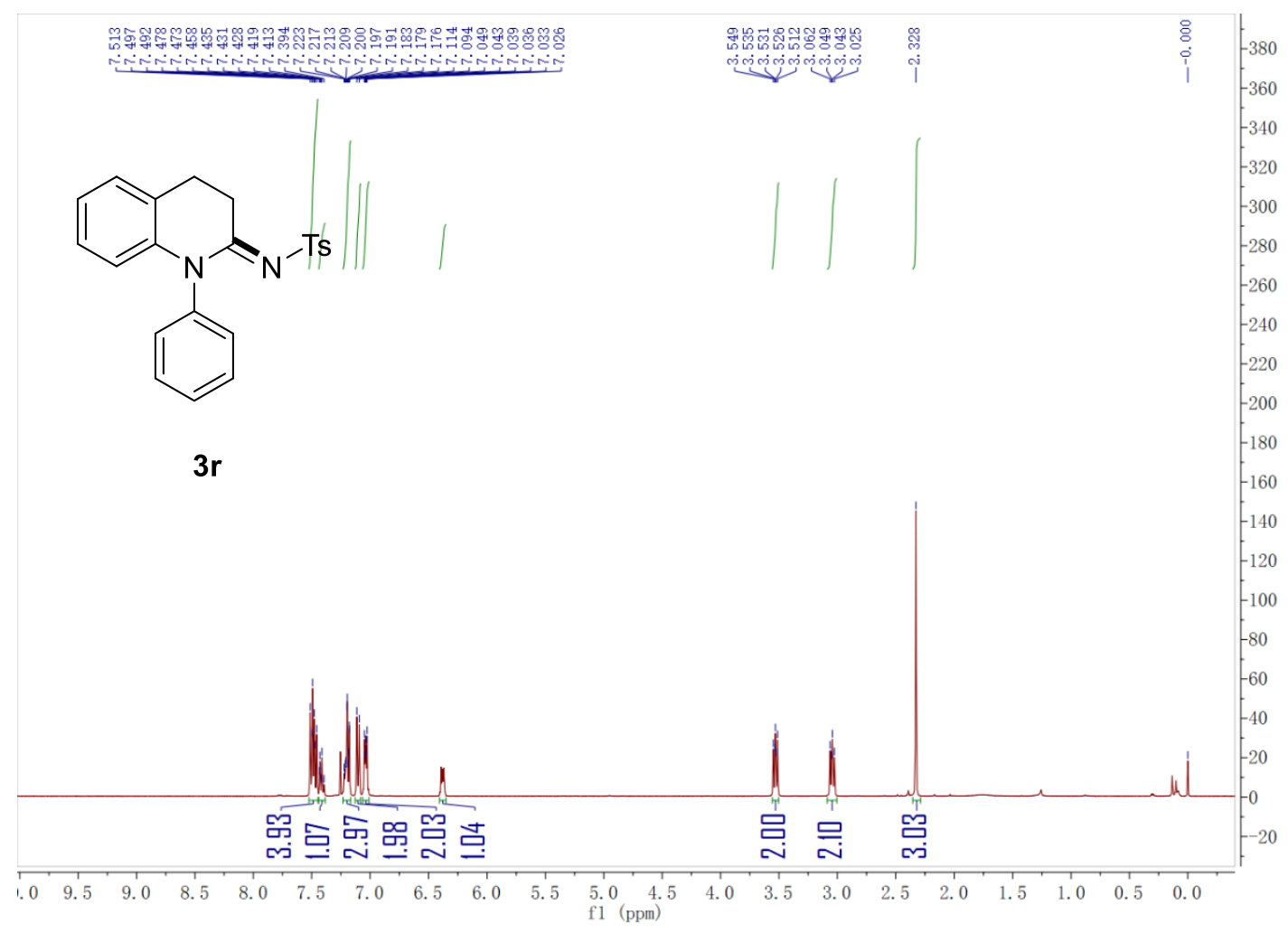

${ }^{1} \mathrm{H}$ NMR spectra $\left(400 \mathrm{MHz}, \mathrm{CDCl}_{3}\right)$ of $\mathbf{3 r}$

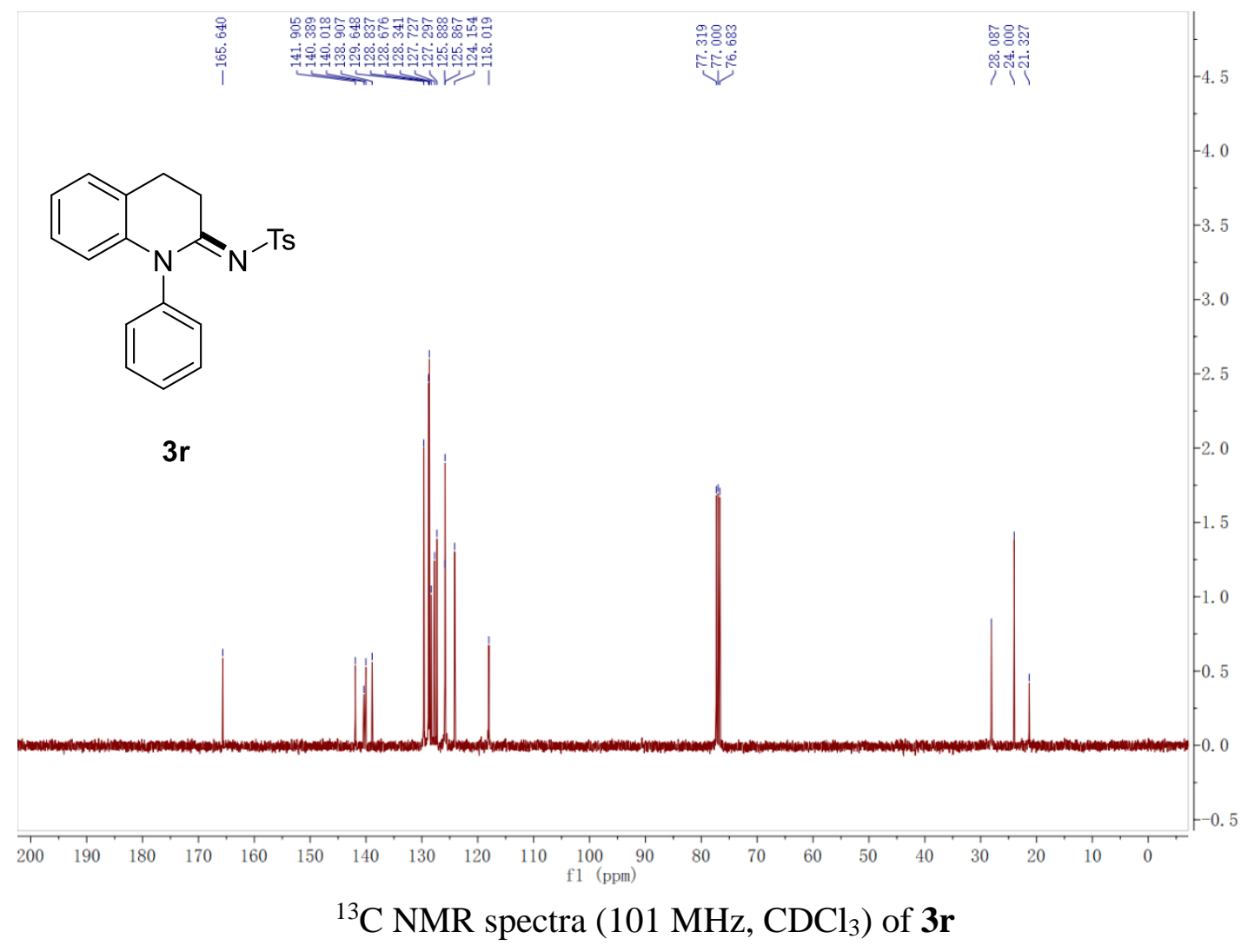




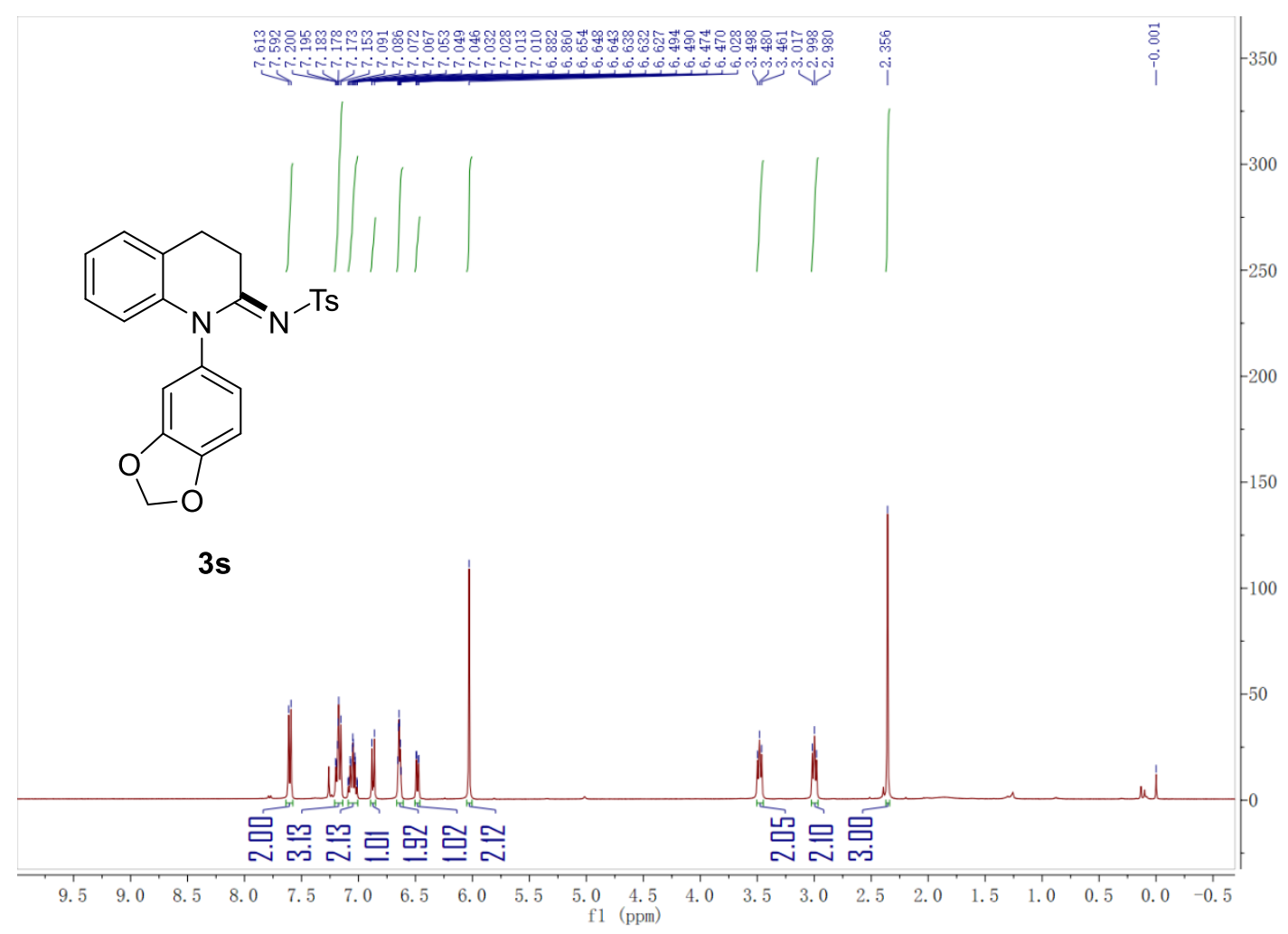

${ }^{1} \mathrm{H}$ NMR spectra (400 MHz, $\mathrm{CDCl}_{3}$ ) of $\mathbf{3 s}$

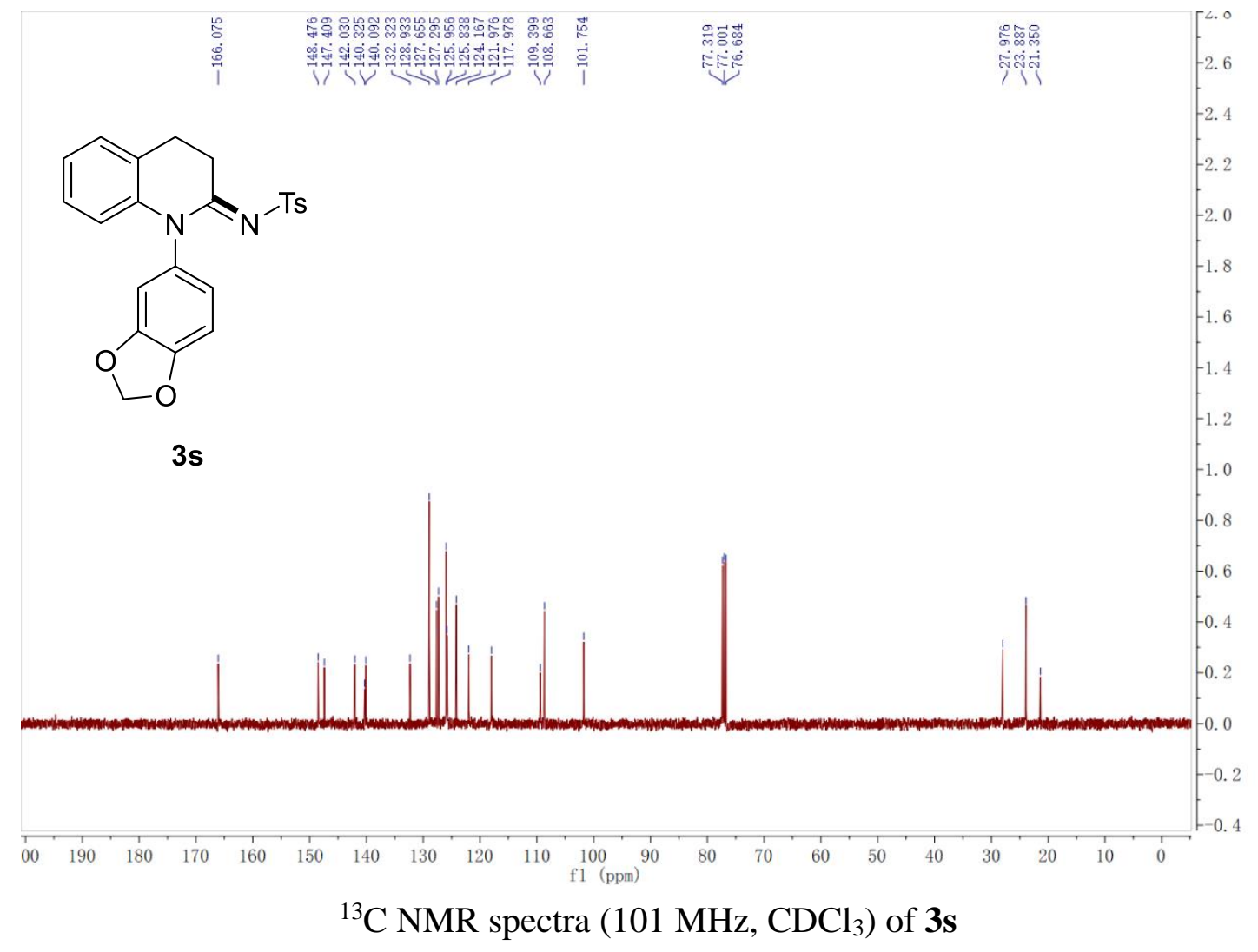




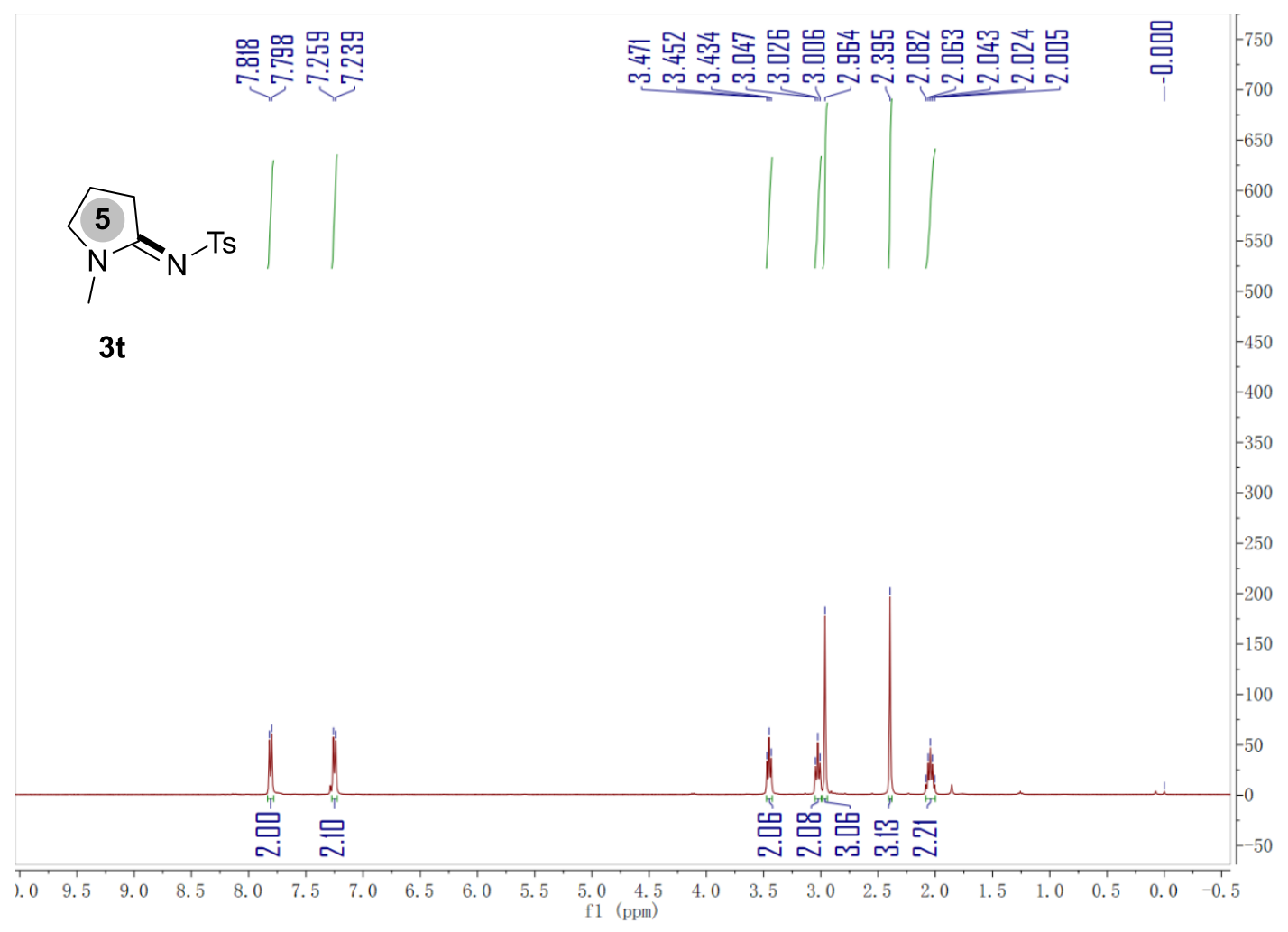

${ }^{1} \mathrm{H}$ NMR spectra $\left(400 \mathrm{MHz}, \mathrm{CDCl}_{3}\right.$ ) of $\mathbf{3 t}$

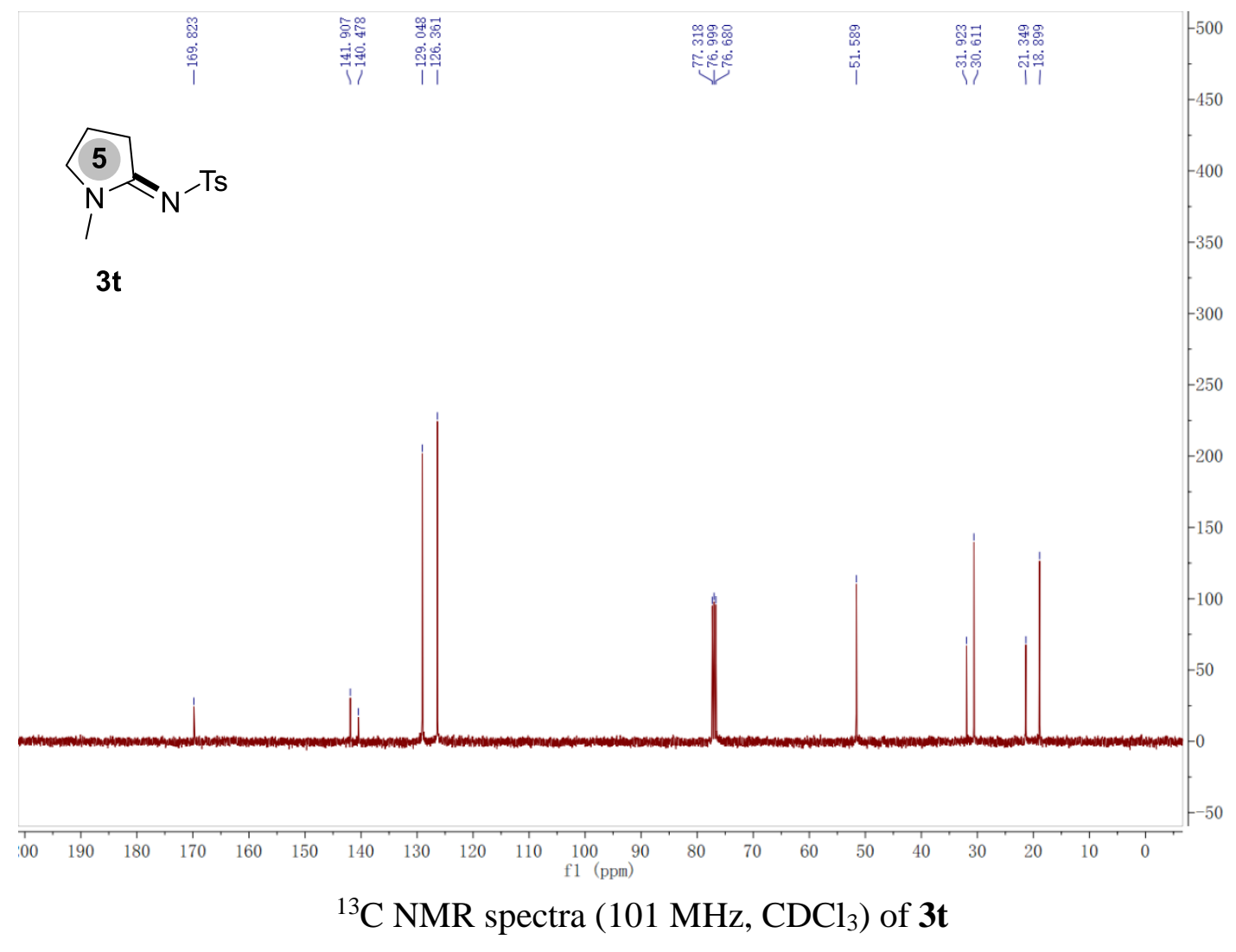




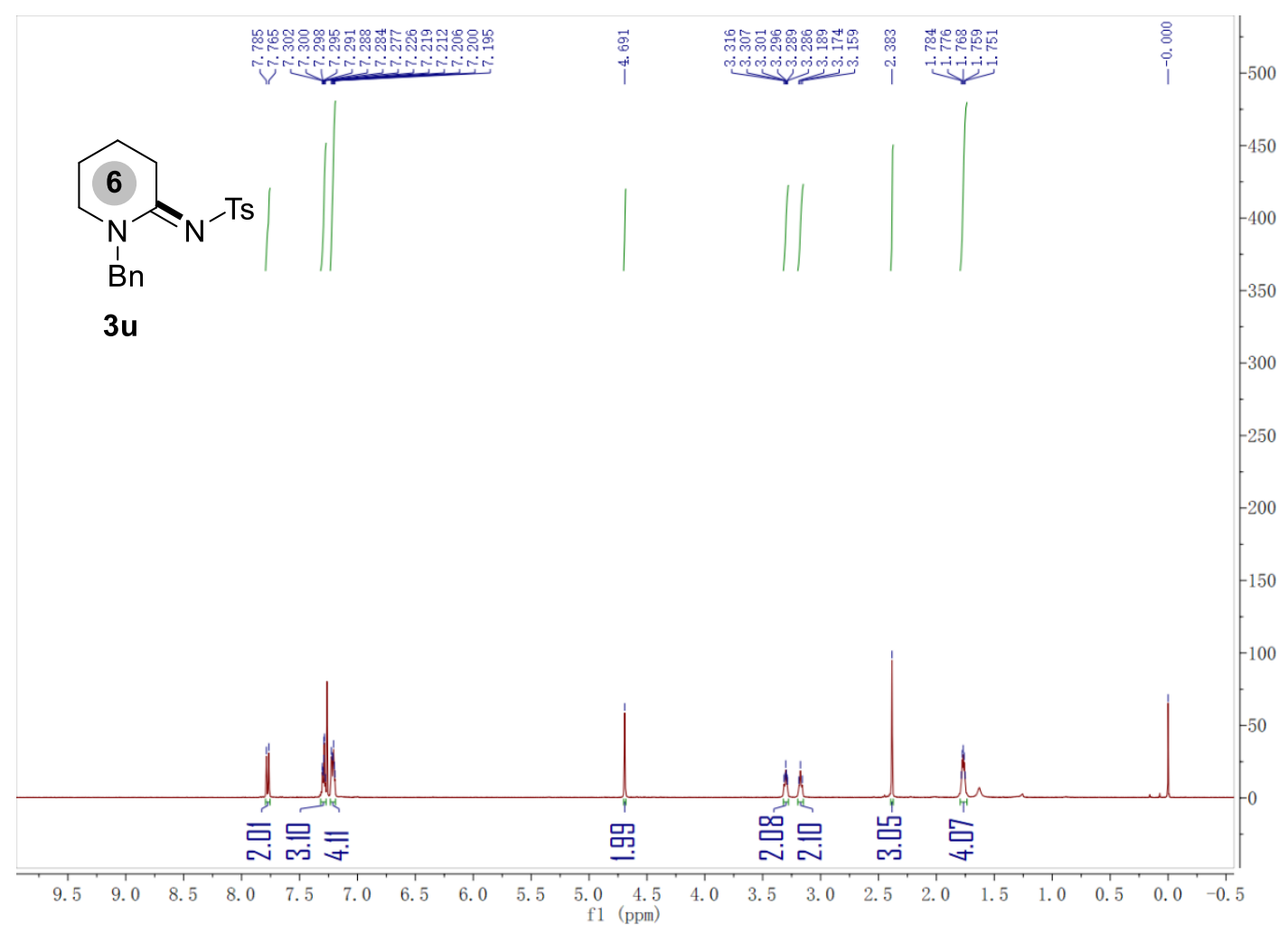

${ }^{1} \mathrm{H}$ NMR spectra $\left(400 \mathrm{MHz}, \mathrm{CDCl}_{3}\right)$ of $\mathbf{3 u}$

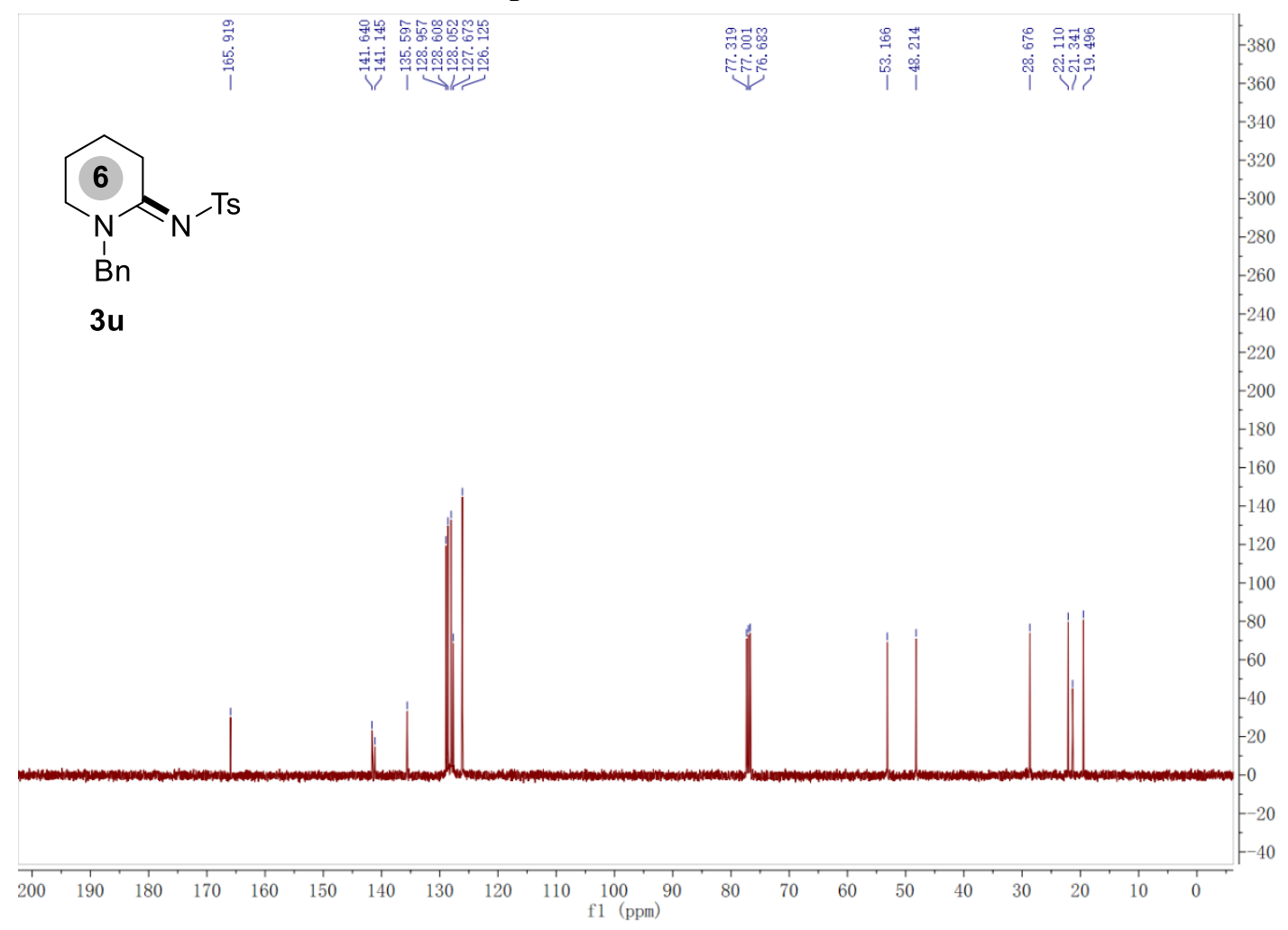

${ }^{13} \mathrm{C}$ NMR spectra $\left(101 \mathrm{MHz}, \mathrm{CDCl}_{3}\right)$ of $\mathbf{3 u}$ 


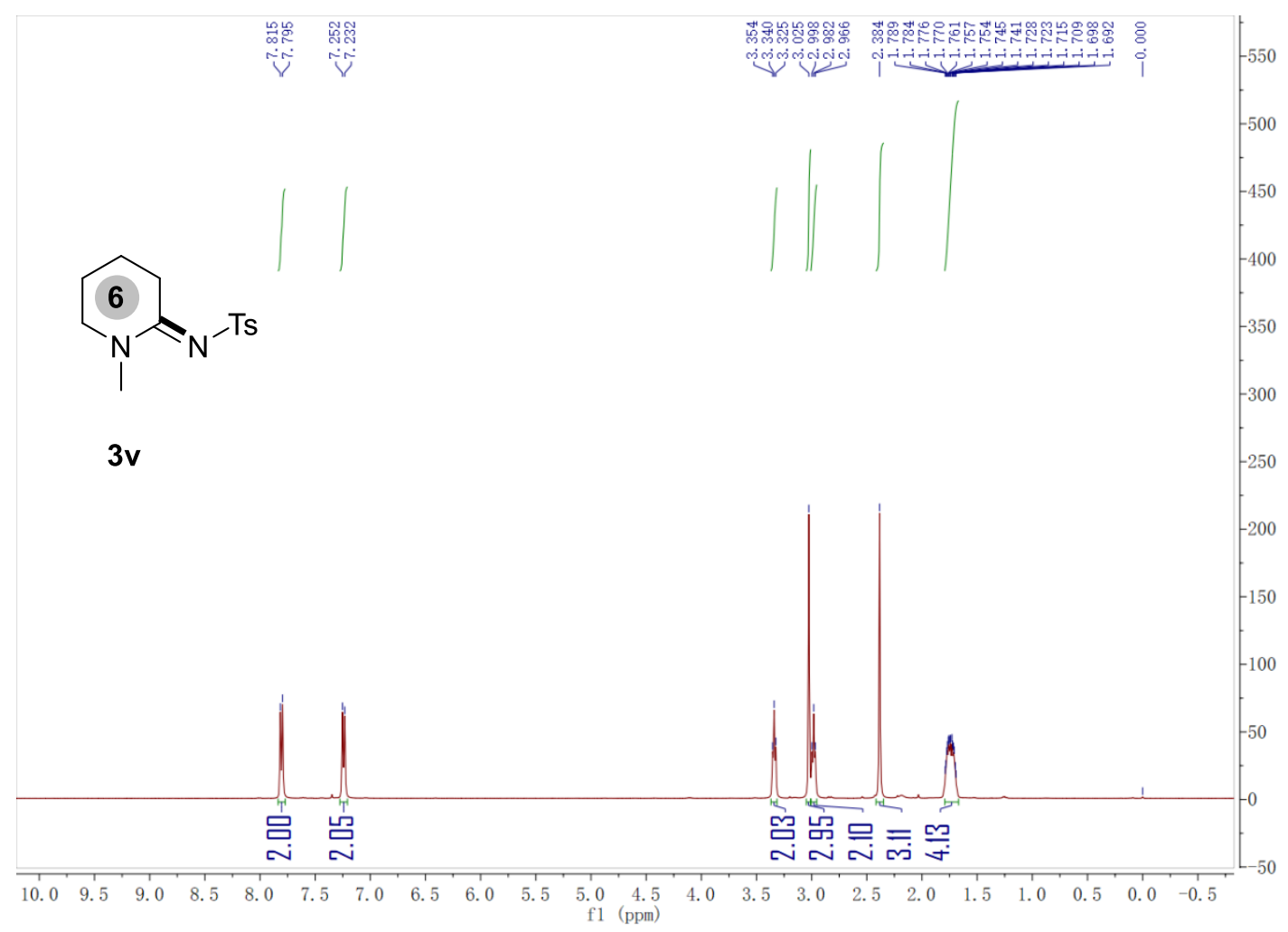

${ }^{1} \mathrm{H}$ NMR spectra $\left(400 \mathrm{MHz}, \mathrm{CDCl}_{3}\right)$ of $\mathbf{3 v}$

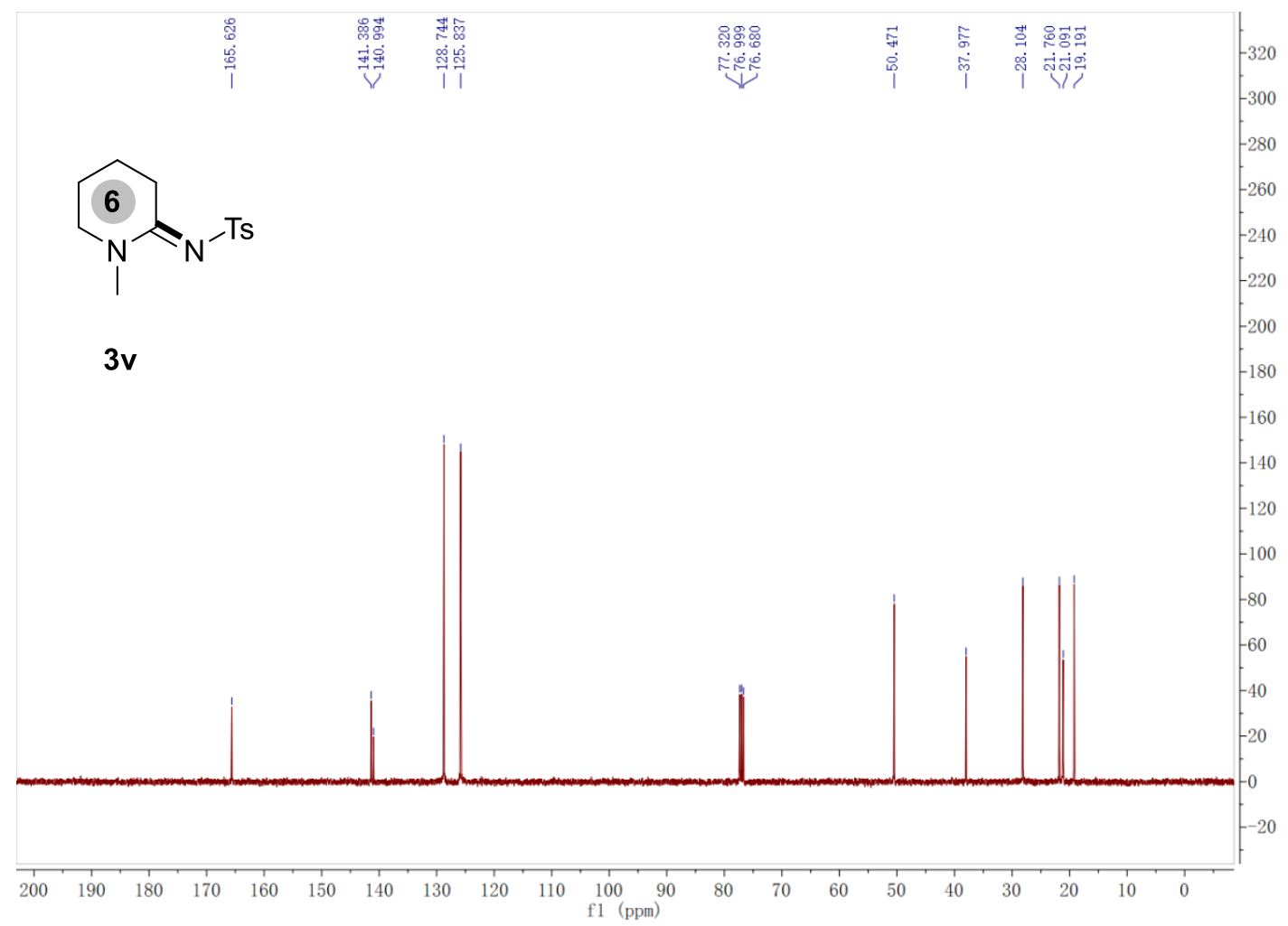

${ }^{13} \mathrm{C}$ NMR spectra $\left(101 \mathrm{MHz}, \mathrm{CDCl}_{3}\right)$ of $\mathbf{3 v}$ 


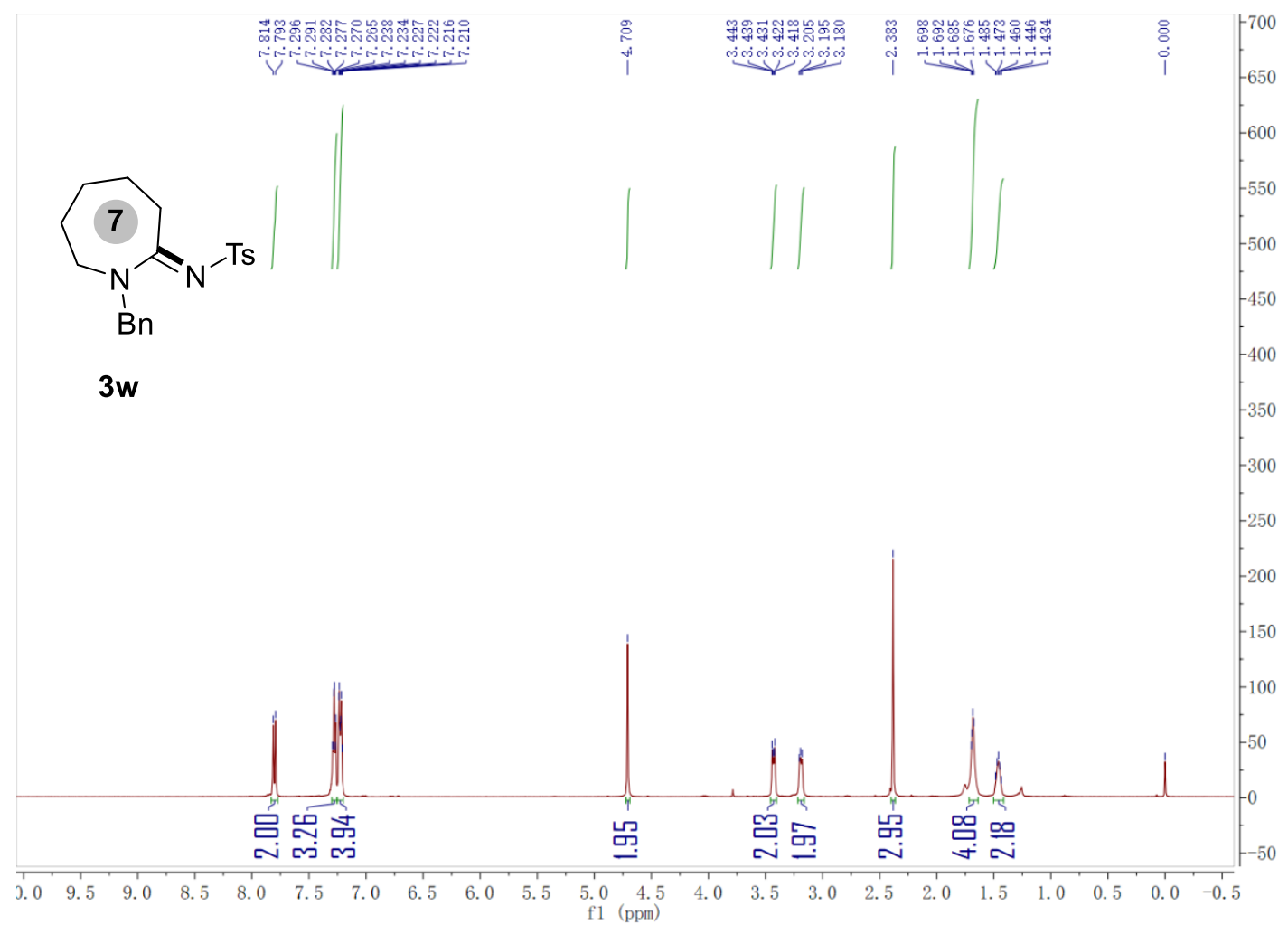

${ }^{1} \mathrm{H}$ NMR spectra $\left(400 \mathrm{MHz}, \mathrm{CDCl}_{3}\right)$ of $\mathbf{3 w}$

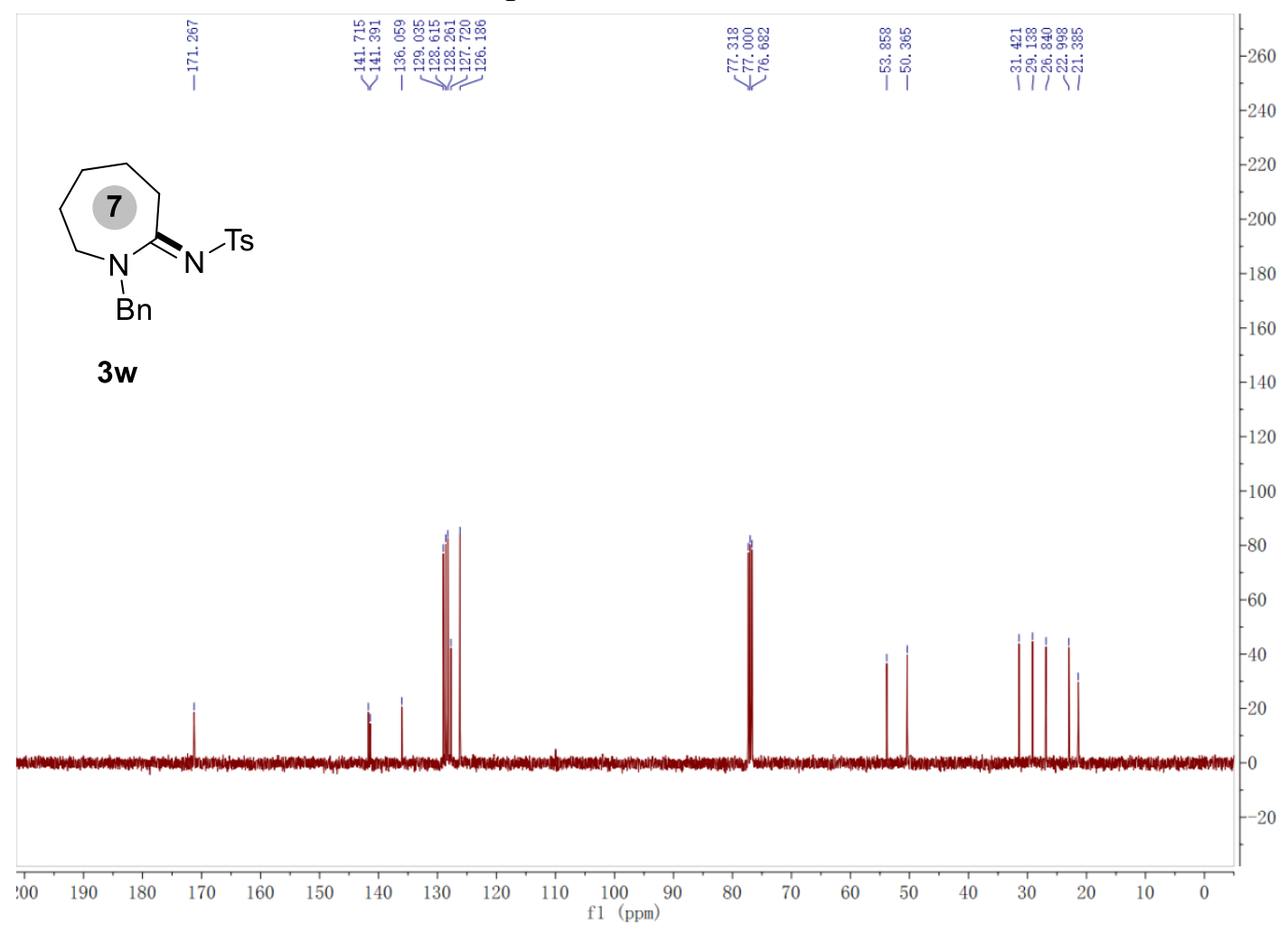

${ }^{13} \mathrm{C}$ NMR spectra $\left(101 \mathrm{MHz}, \mathrm{CDCl}_{3}\right)$ of $\mathbf{3 w}$ 


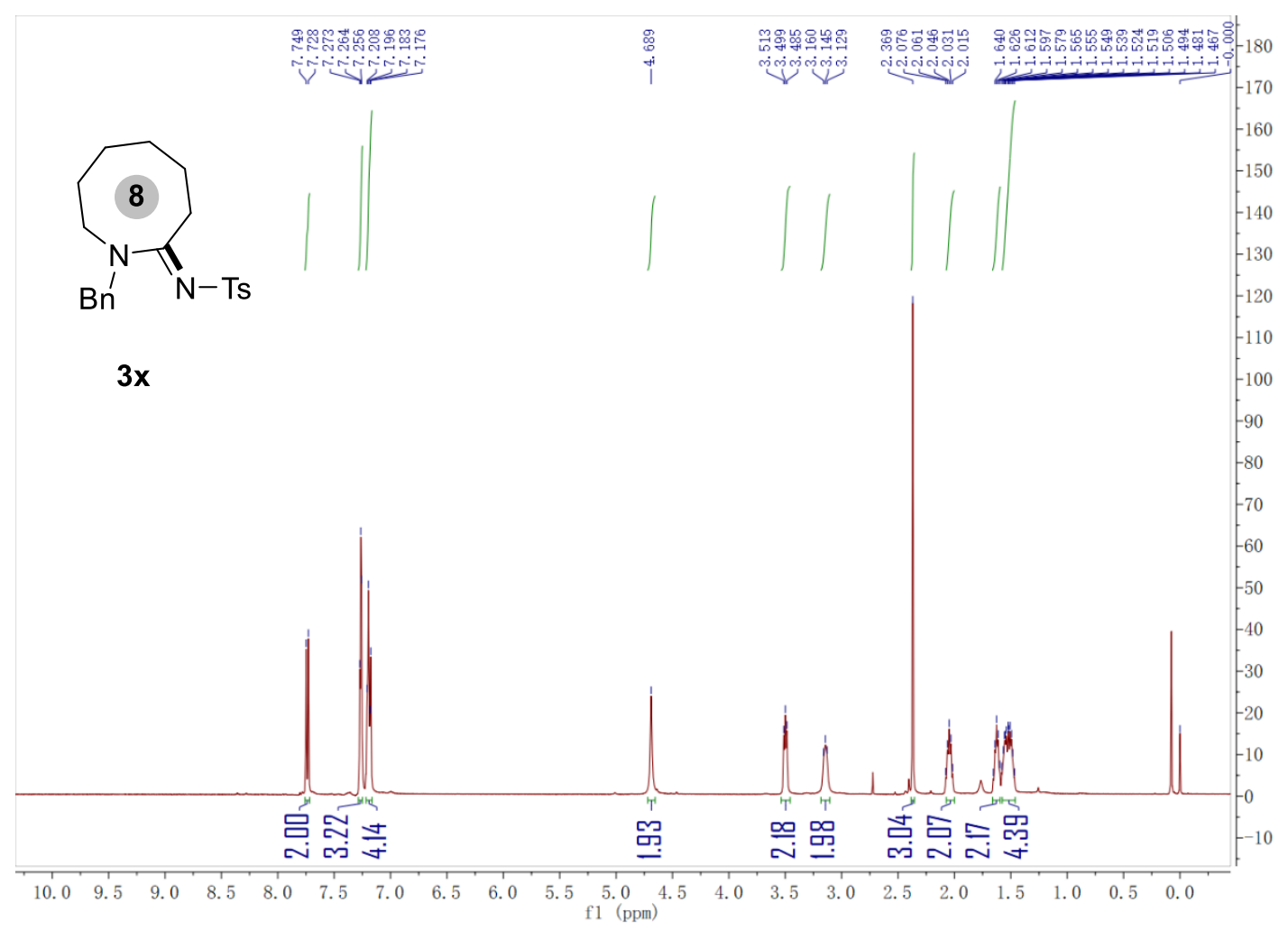

${ }^{1} \mathrm{H}$ NMR spectra $\left(400 \mathrm{MHz}, \mathrm{CDCl}_{3}\right)$ of $\mathbf{3 x}$

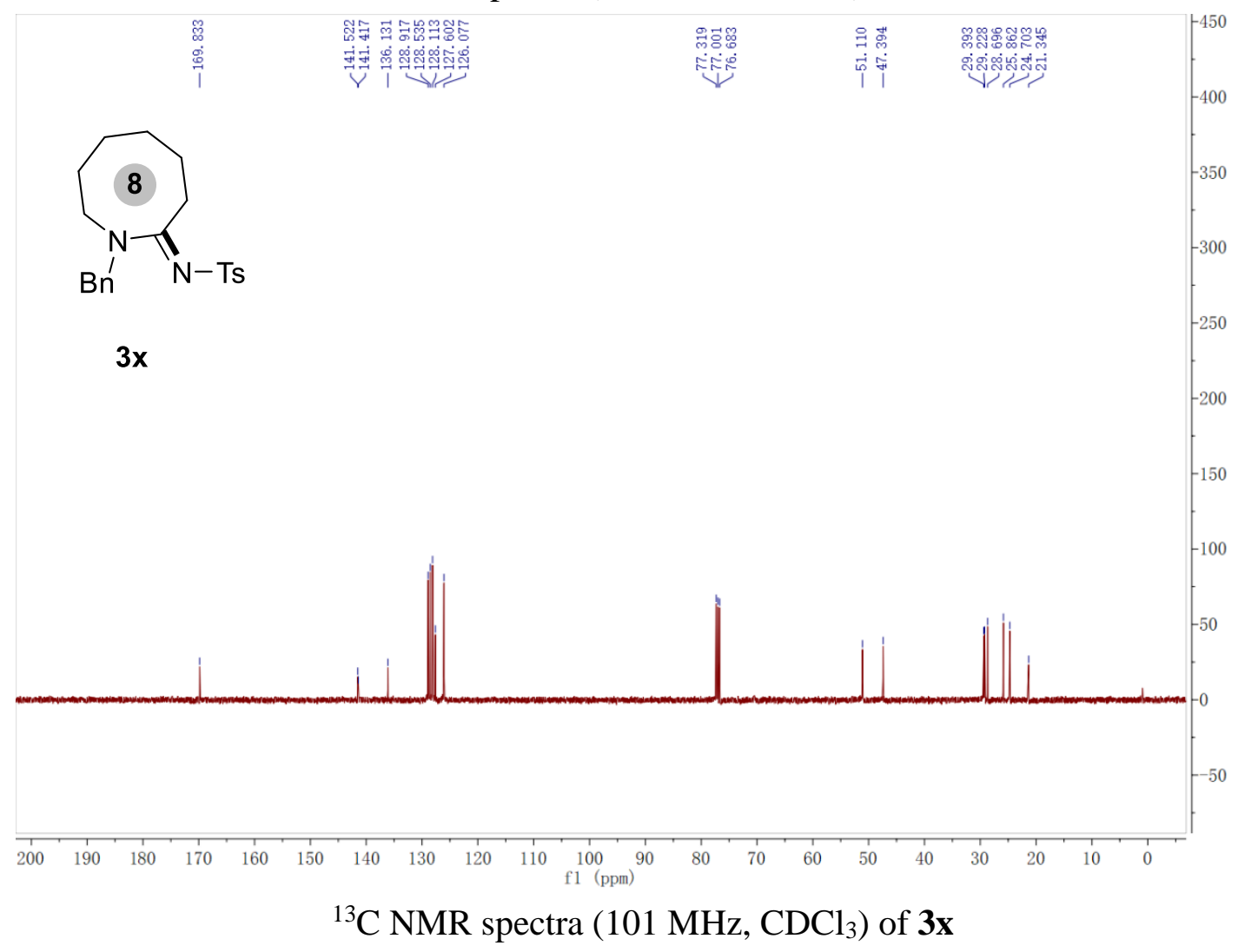




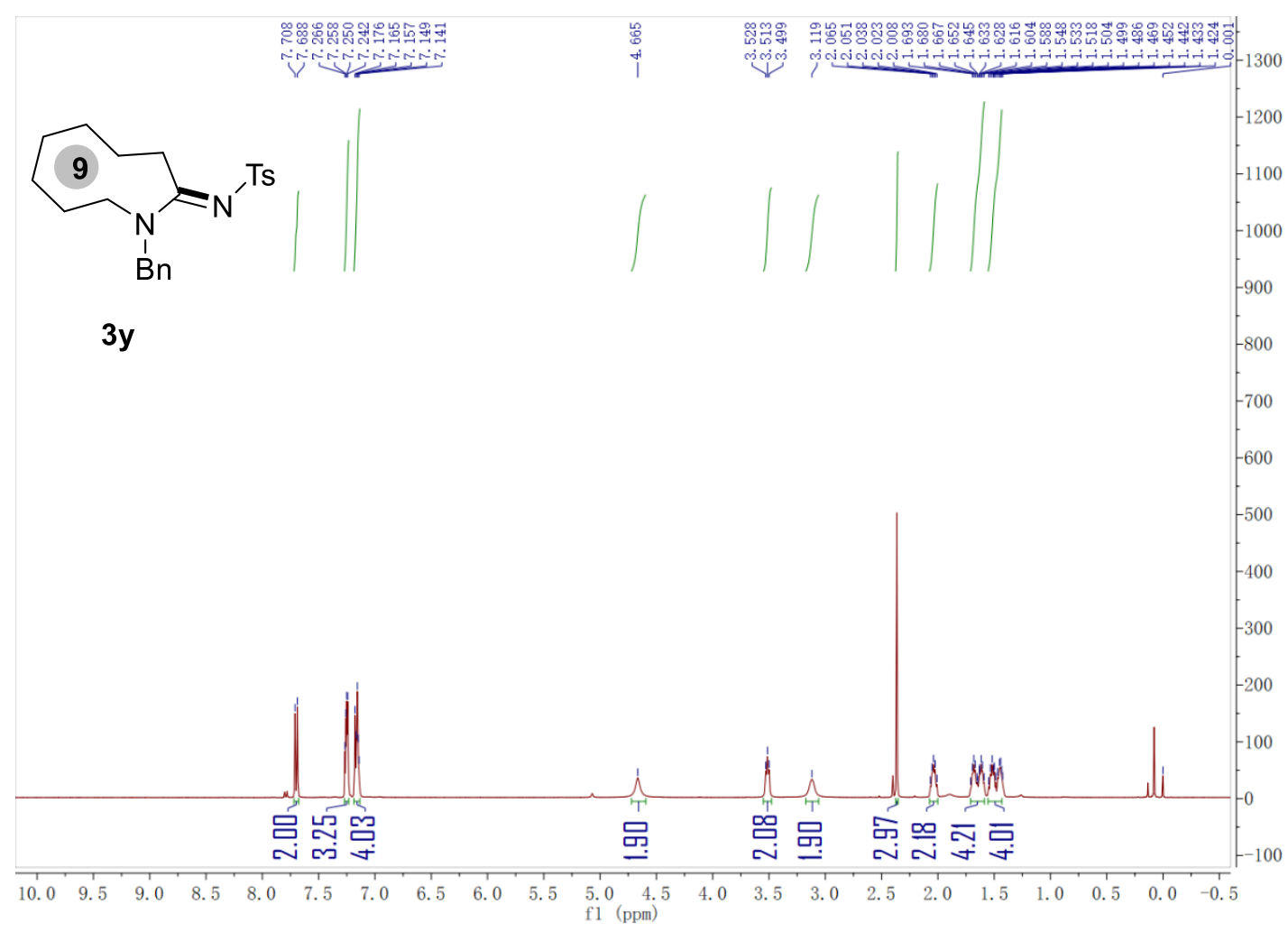

${ }^{1} \mathrm{H}$ NMR spectra (400 MHz, $\mathrm{CDCl}_{3}$ ) of $\mathbf{3 y}$

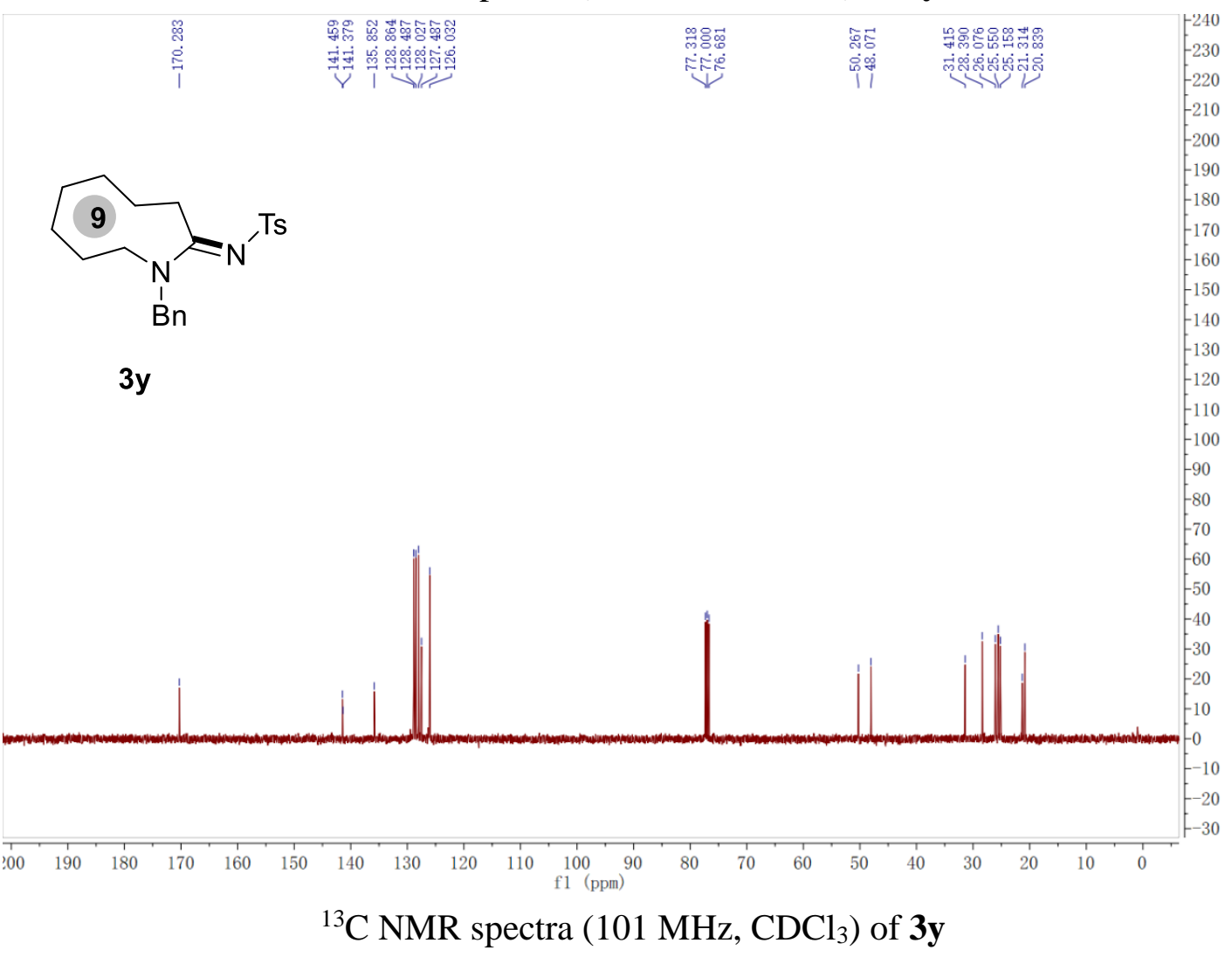




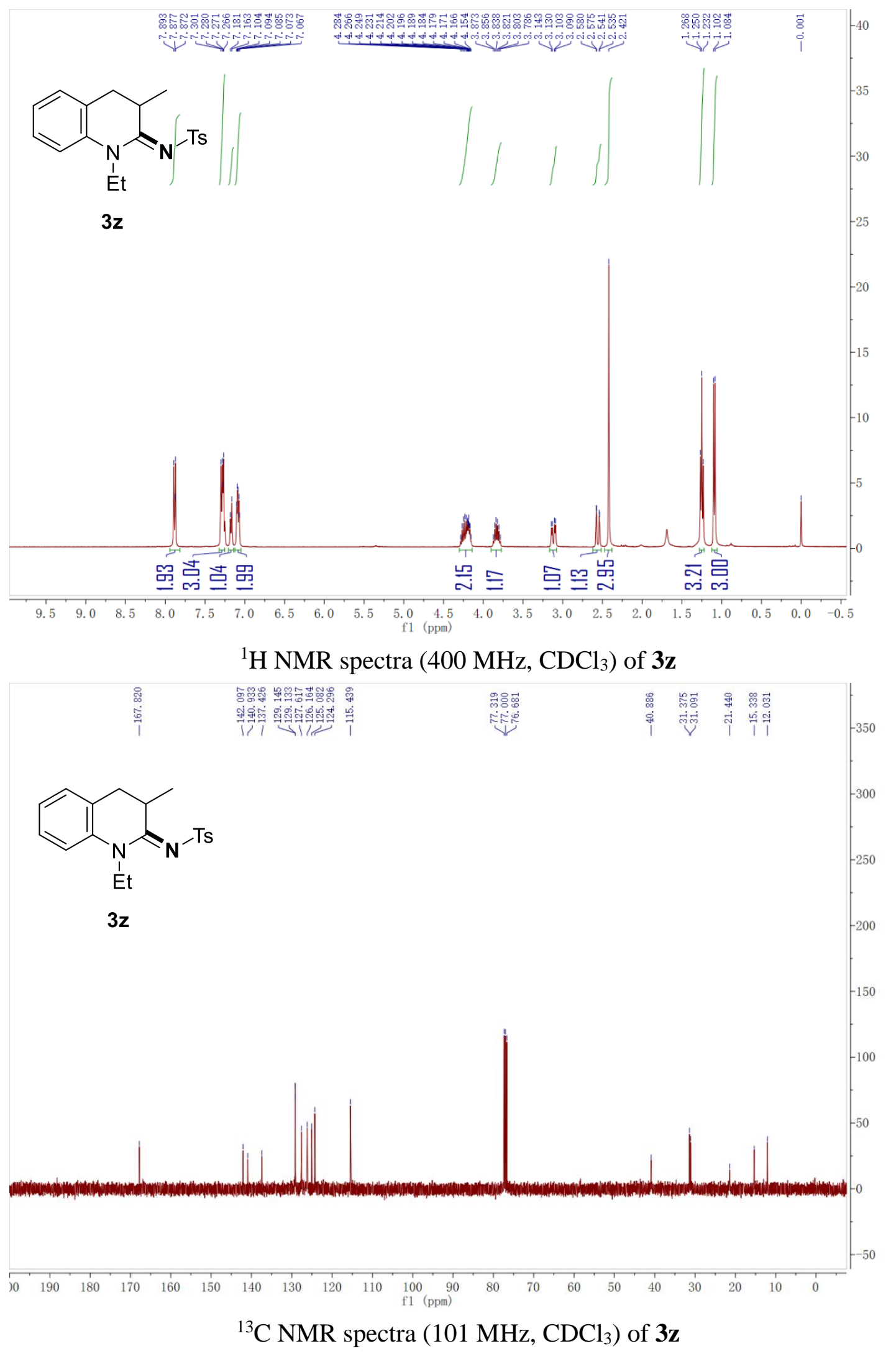




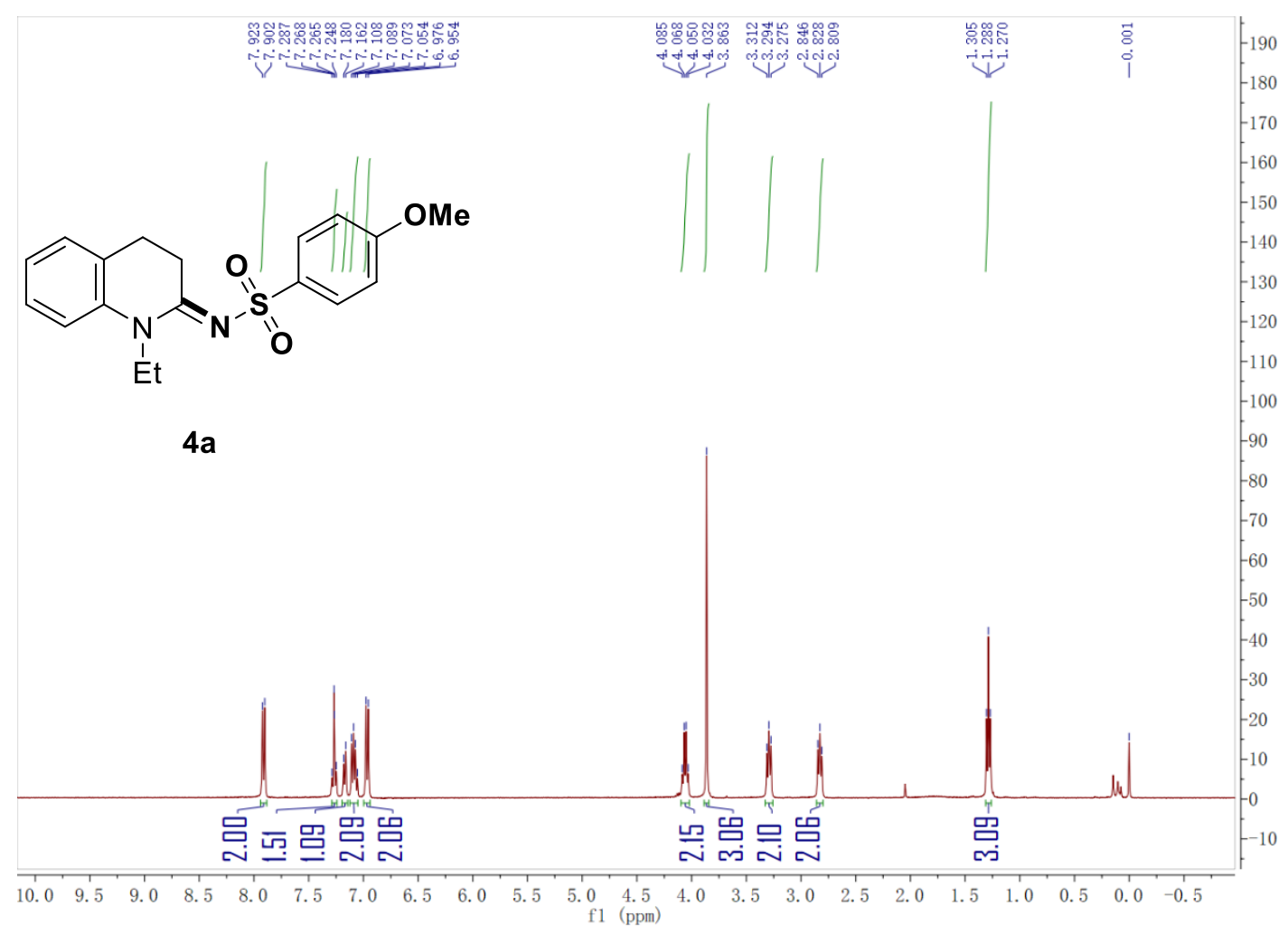

${ }^{1} \mathrm{H}$ NMR spectra (400 MHz, $\mathrm{CDCl}_{3}$ ) of $\mathbf{4 a}$

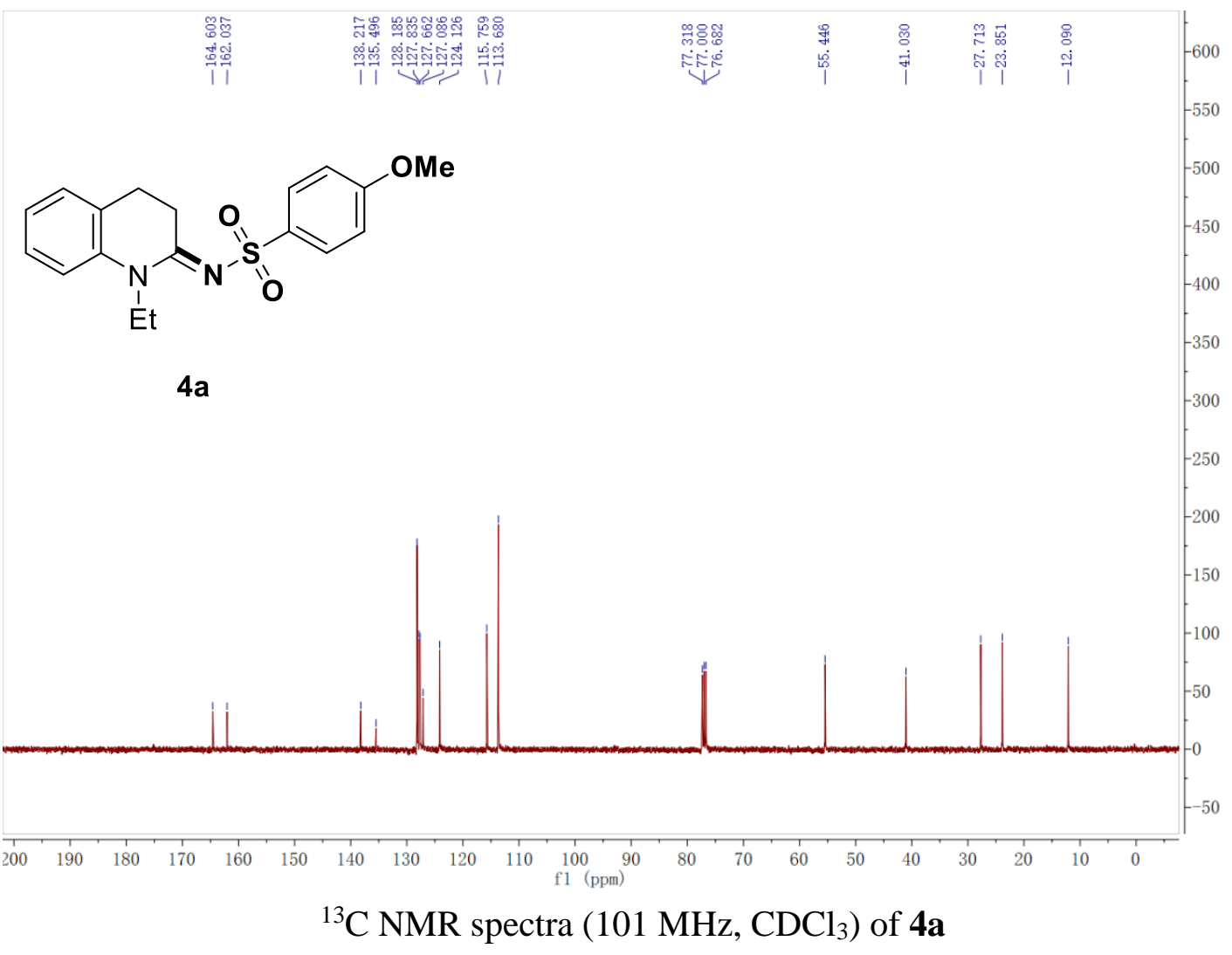




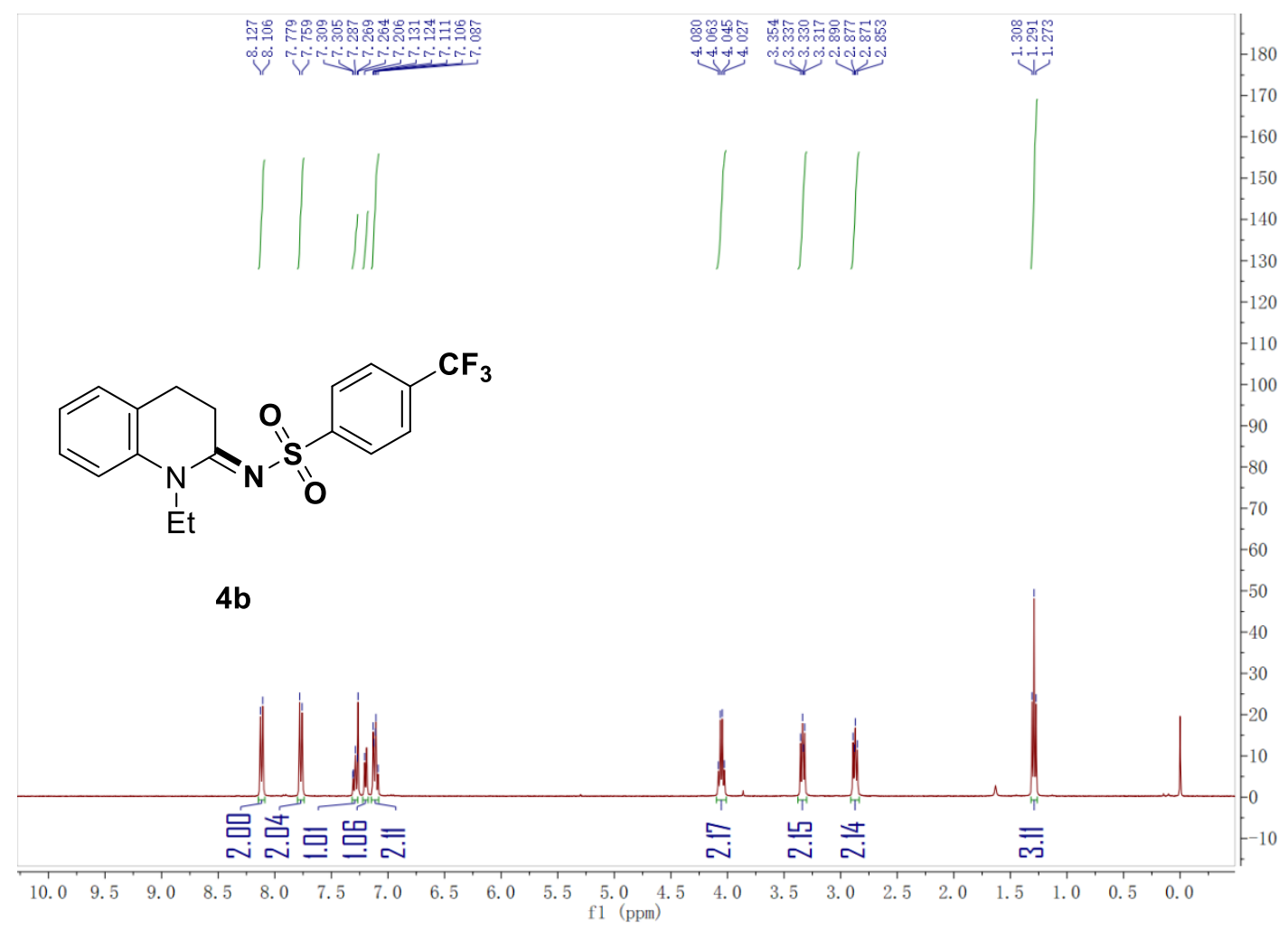

${ }^{1} \mathrm{H}$ NMR spectra $\left(400 \mathrm{MHz}, \mathrm{CDCl}_{3}\right.$ ) of $\mathbf{4 b}$

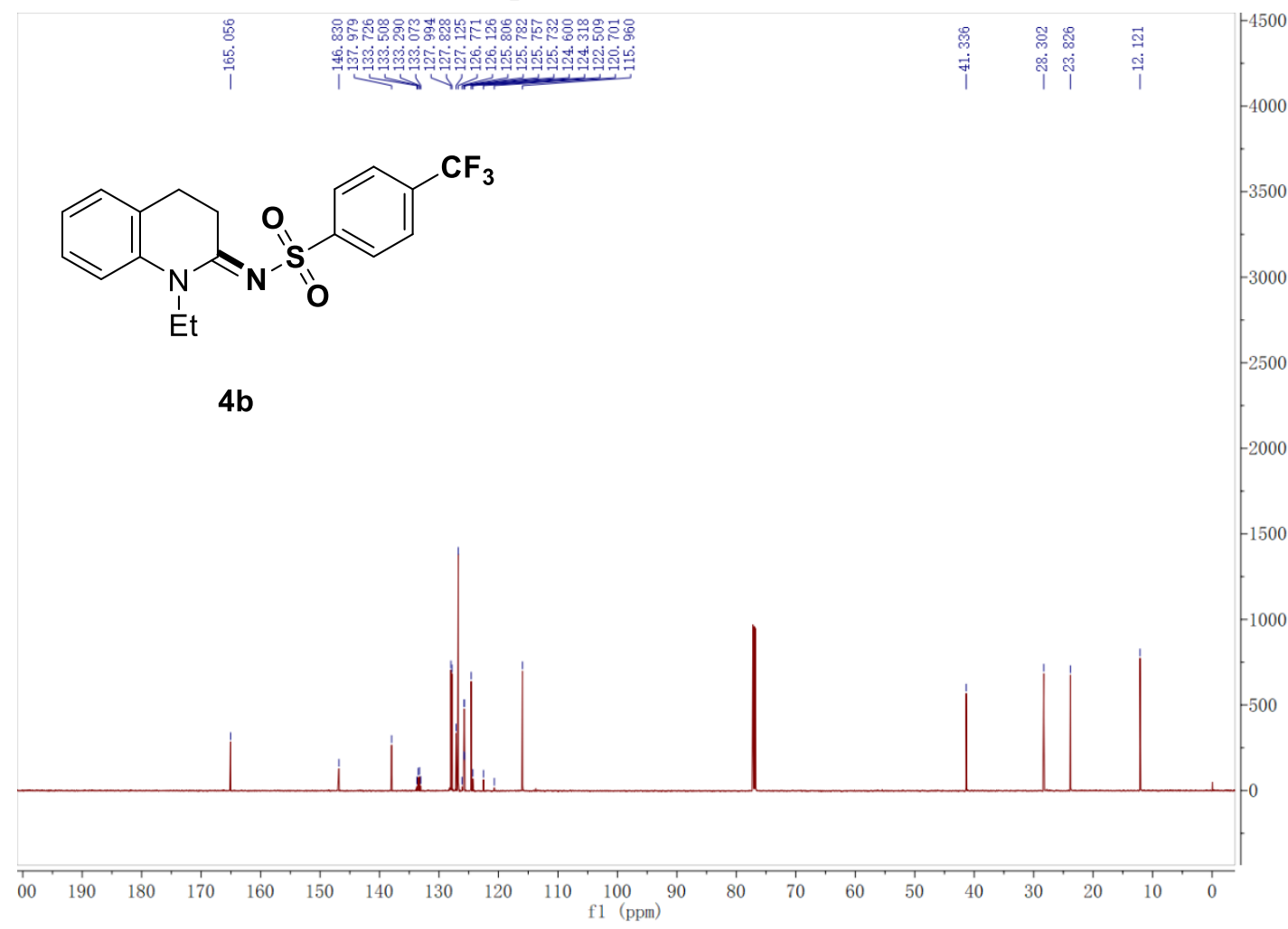

${ }^{13} \mathrm{C}$ NMR spectra $\left(151 \mathrm{MHz}, \mathrm{CDCl}_{3}\right)$ of $\mathbf{4 b}$ 


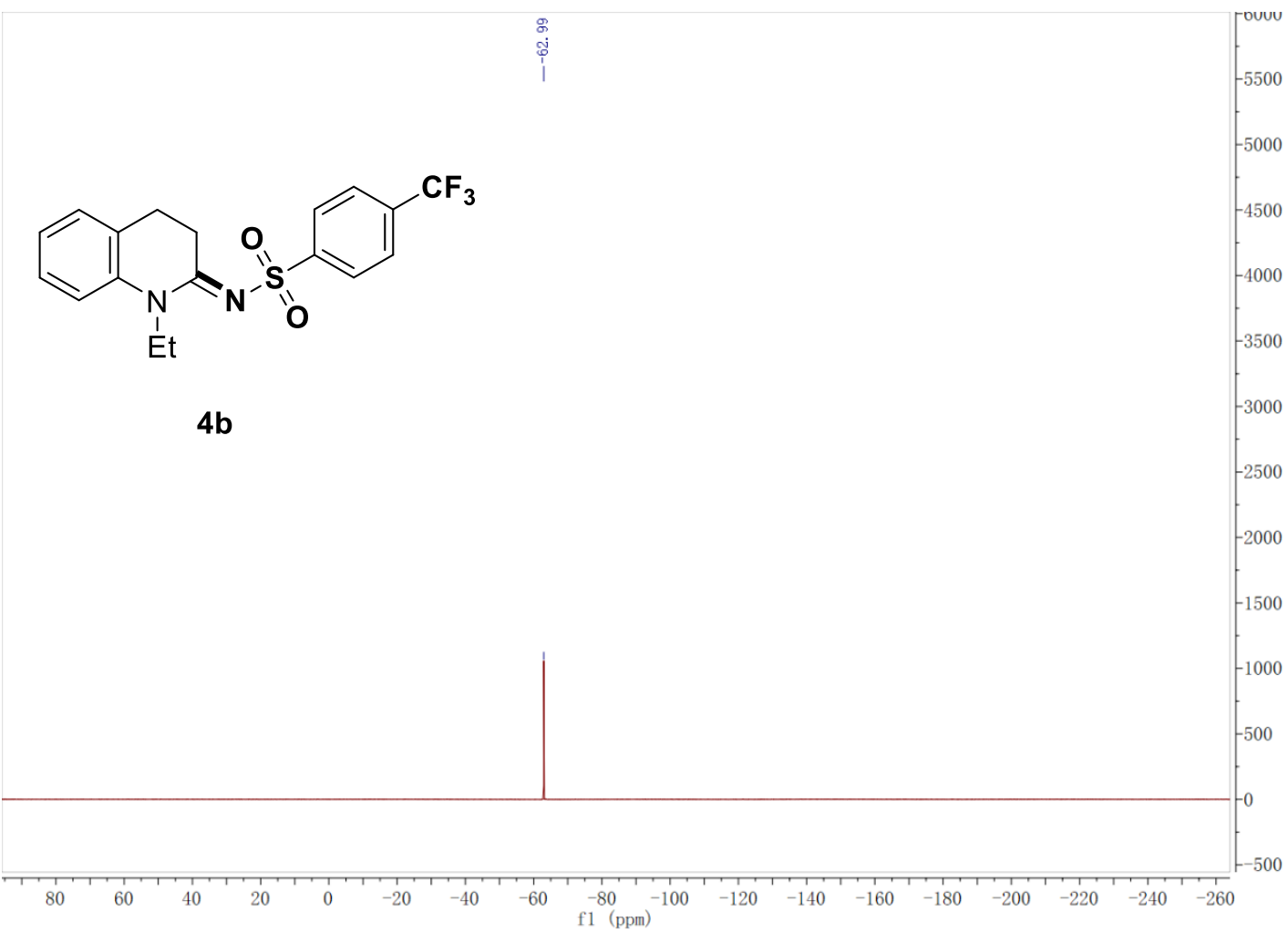

${ }^{19} \mathrm{~F}$ NMR spectra $\left(376 \mathrm{MHz}, \mathrm{CDCl}_{3}\right)$ of $\mathbf{4 b}$ 


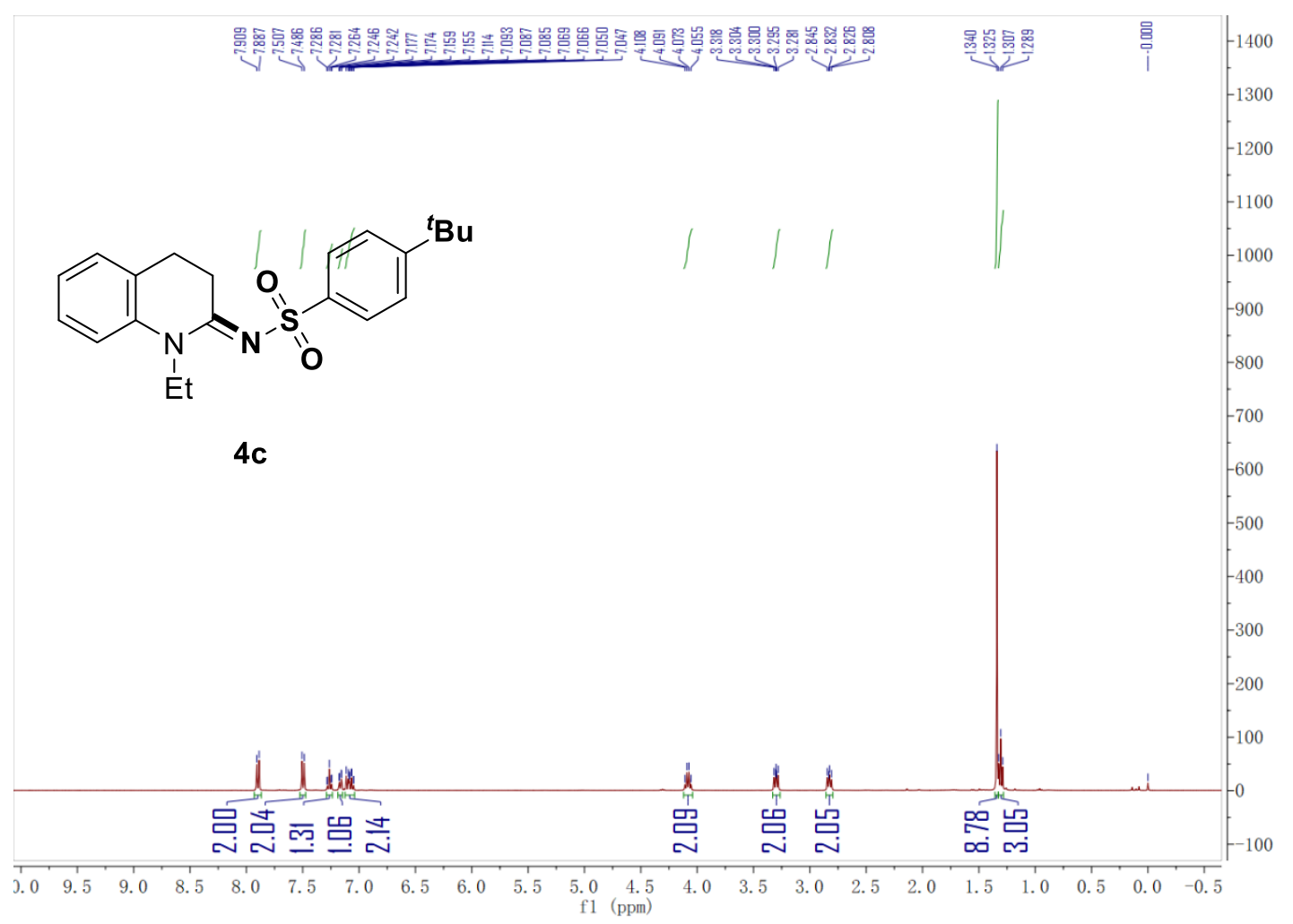

${ }^{1} \mathrm{H}$ NMR spectra $\left(400 \mathrm{MHz}, \mathrm{CDCl}_{3}\right)$ of $\mathbf{4 c}$

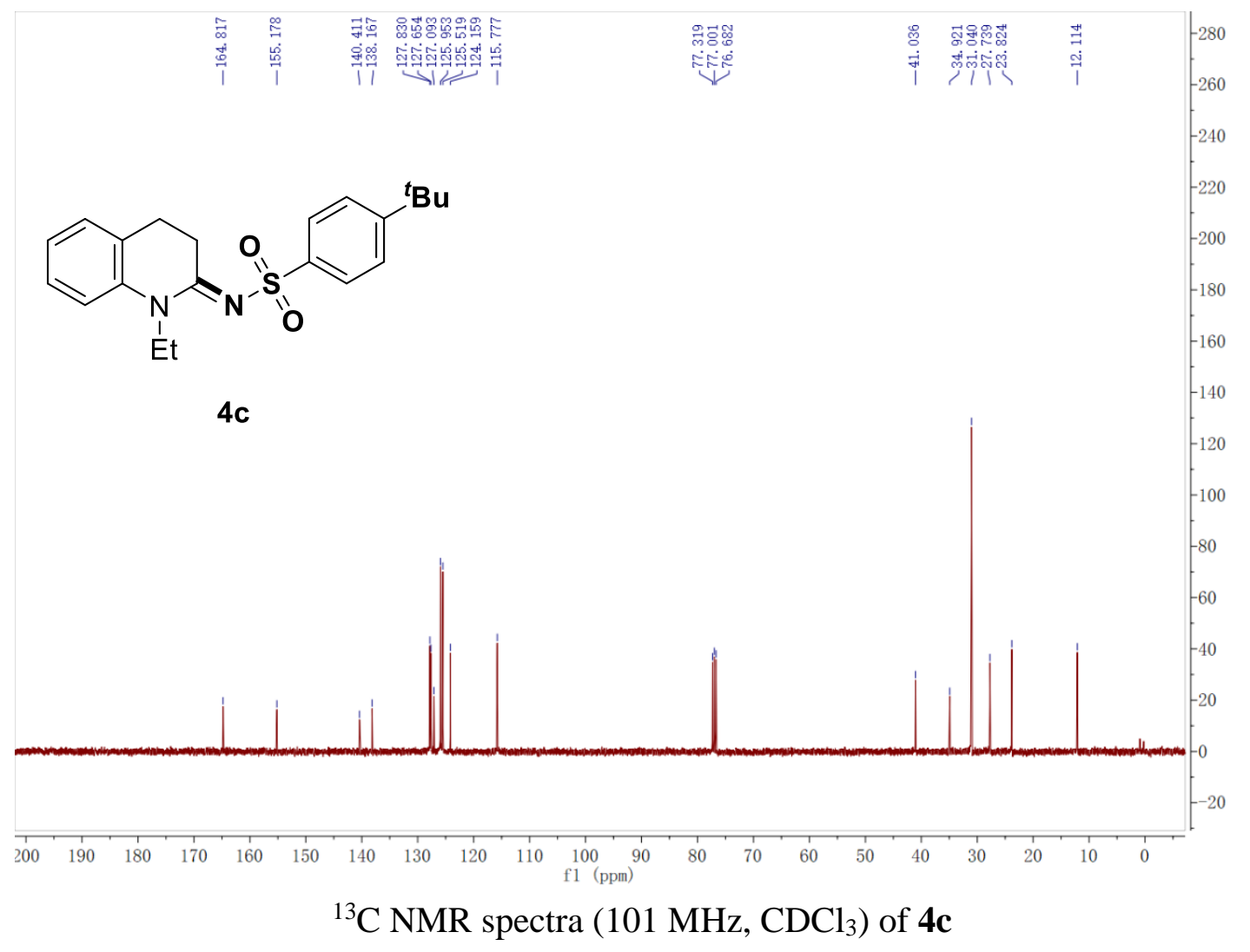




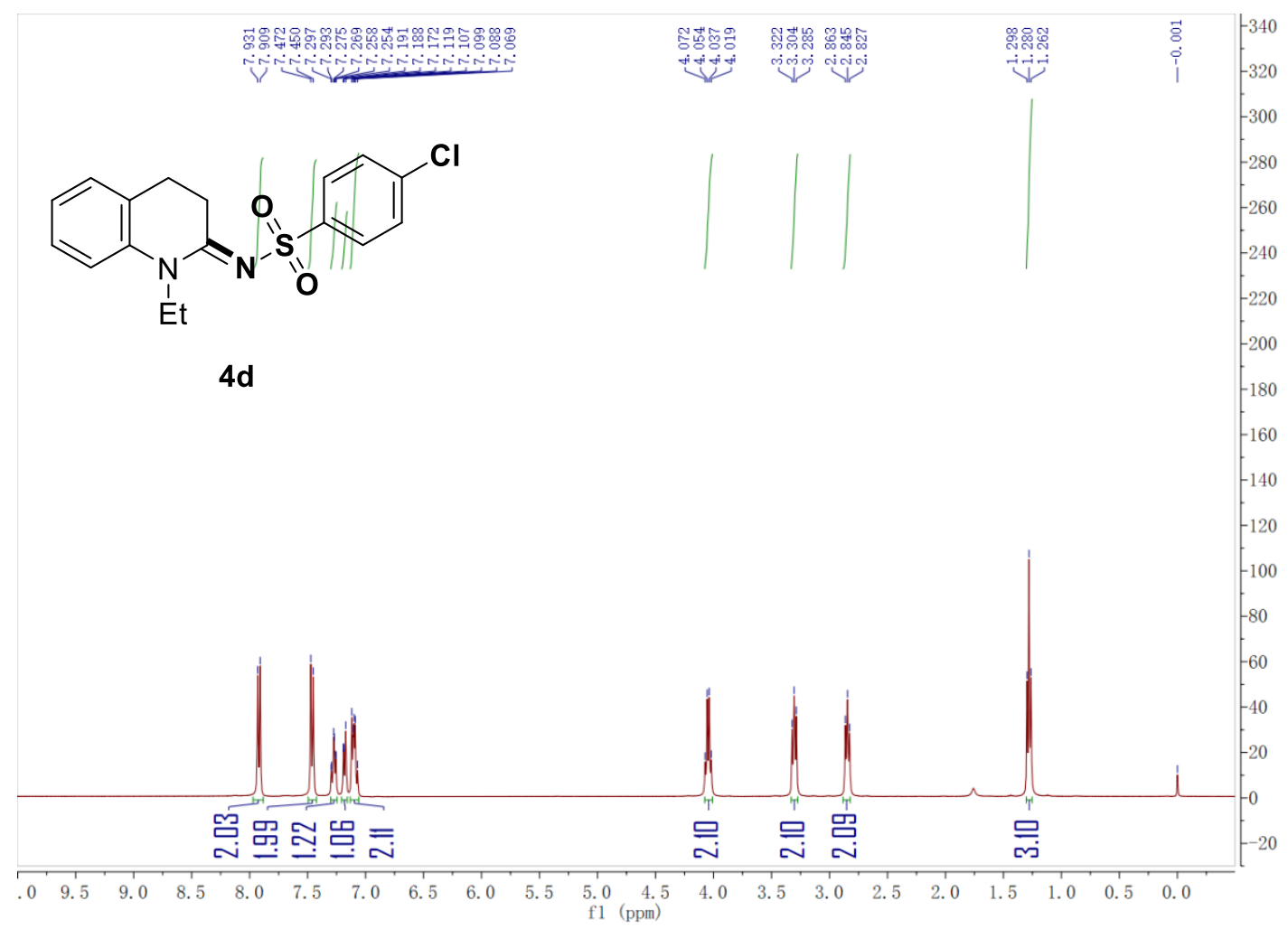

${ }^{1} \mathrm{H}$ NMR spectra $\left(400 \mathrm{MHz}, \mathrm{CDCl}_{3}\right)$ of $\mathbf{4 d}$

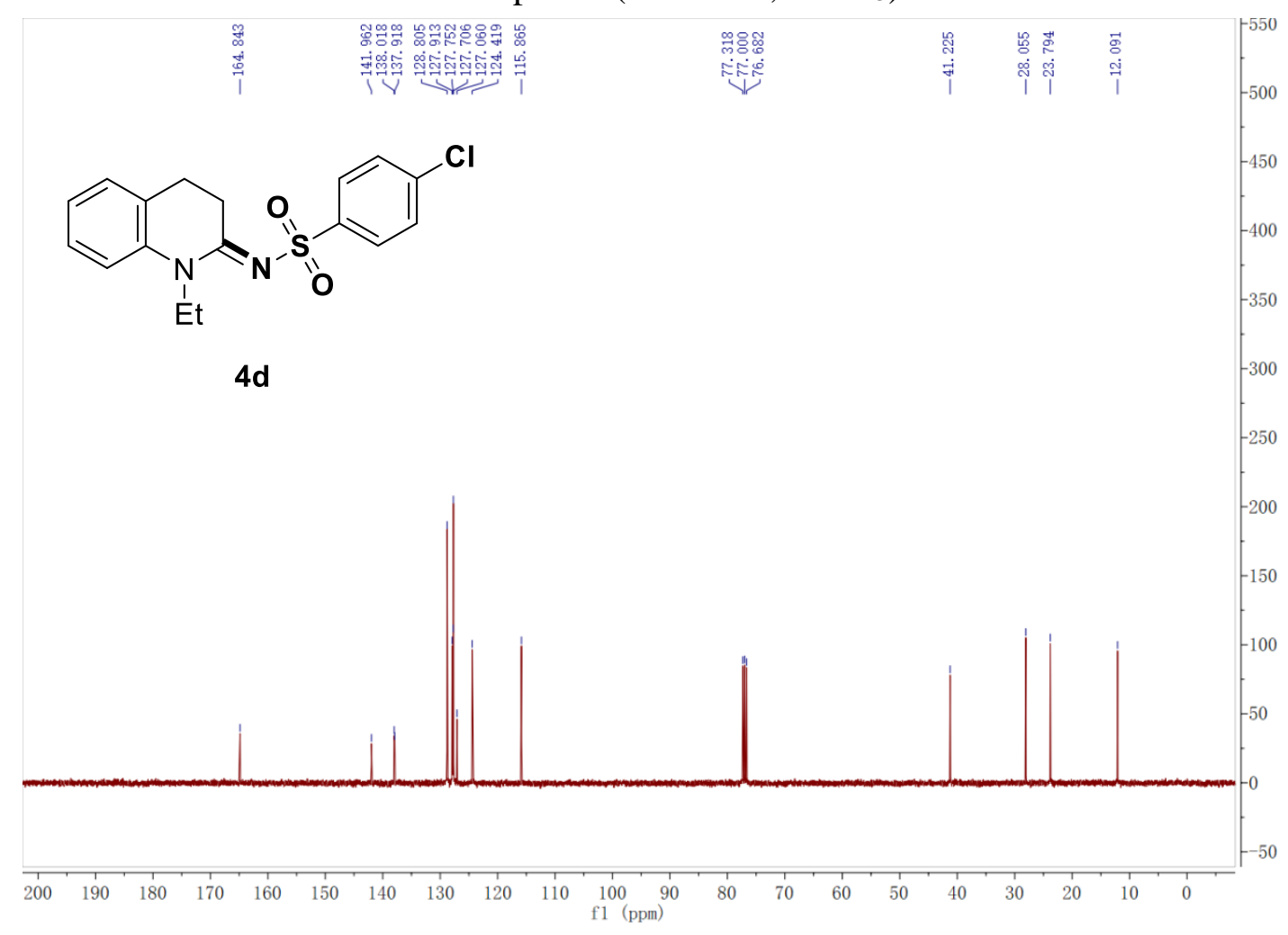

${ }^{13} \mathrm{C}$ NMR spectra $\left(101 \mathrm{MHz}, \mathrm{CDCl}_{3}\right)$ of $\mathbf{4 d}$ 


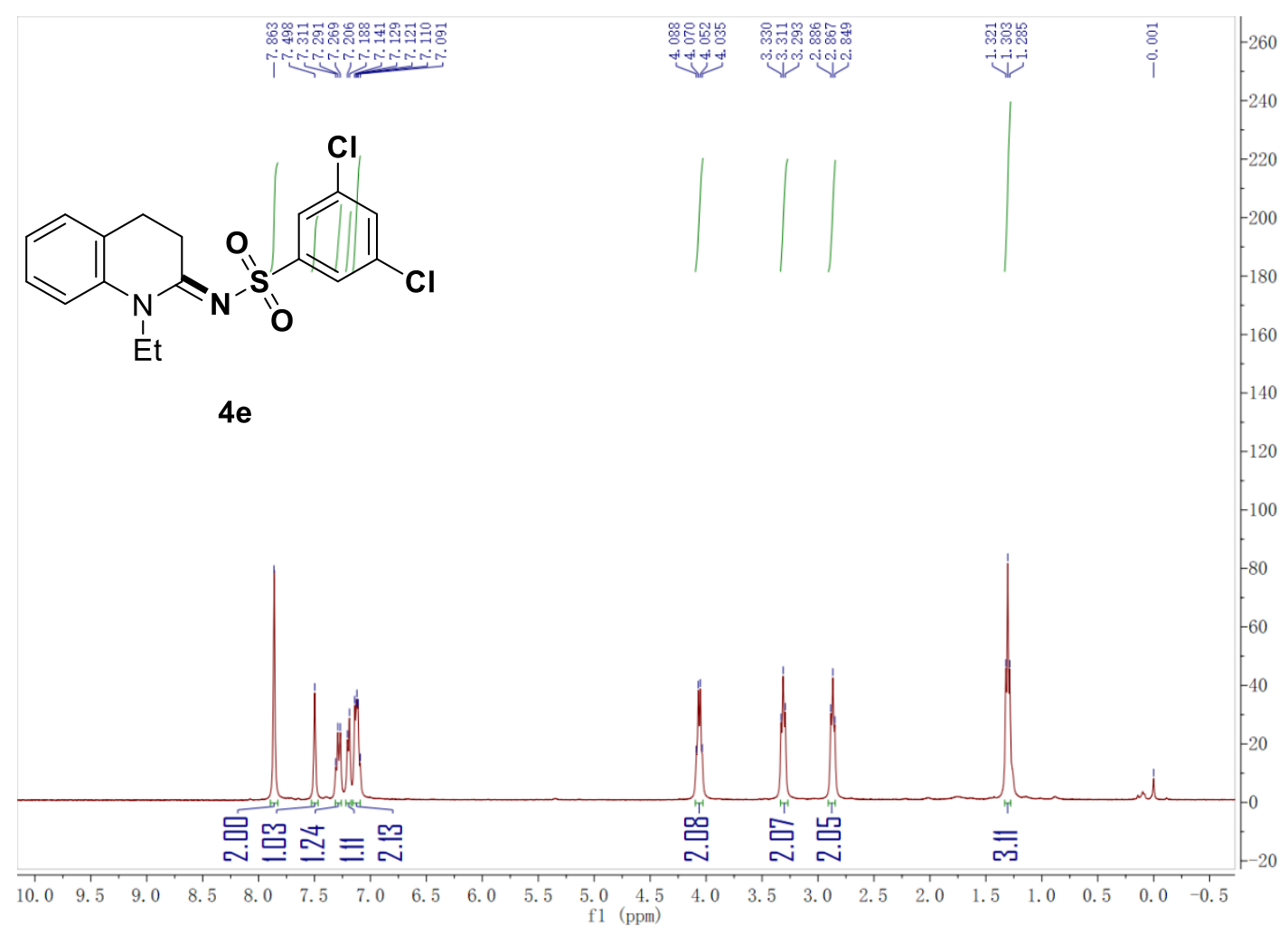

${ }^{1} \mathrm{H}$ NMR spectra $\left(400 \mathrm{MHz}, \mathrm{CDCl}_{3}\right)$ of $\mathbf{4 e}$

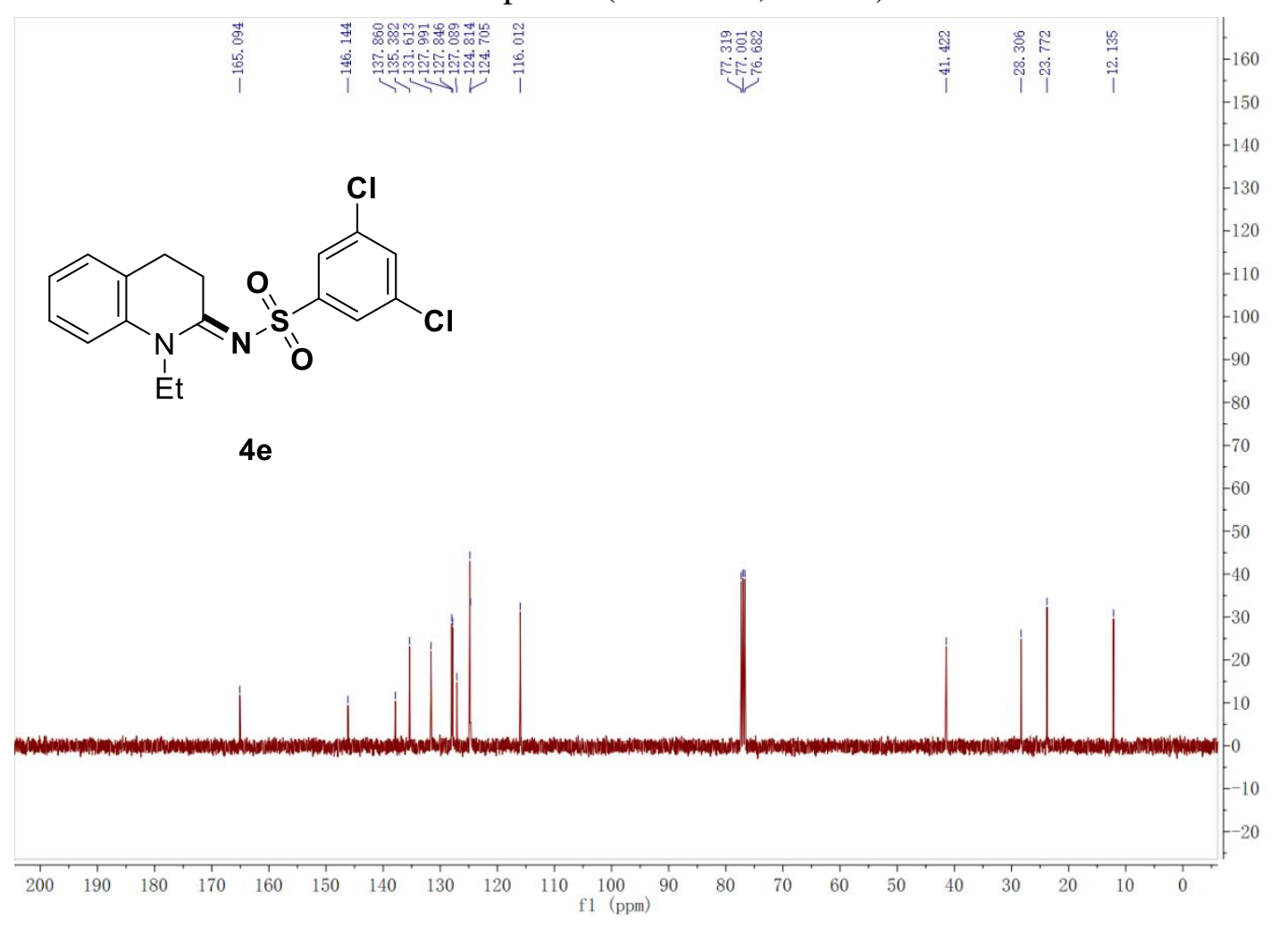

${ }^{13} \mathrm{C}$ NMR spectra $\left(101 \mathrm{MHz}, \mathrm{CDCl}_{3}\right)$ of $\mathbf{4 e}$ 


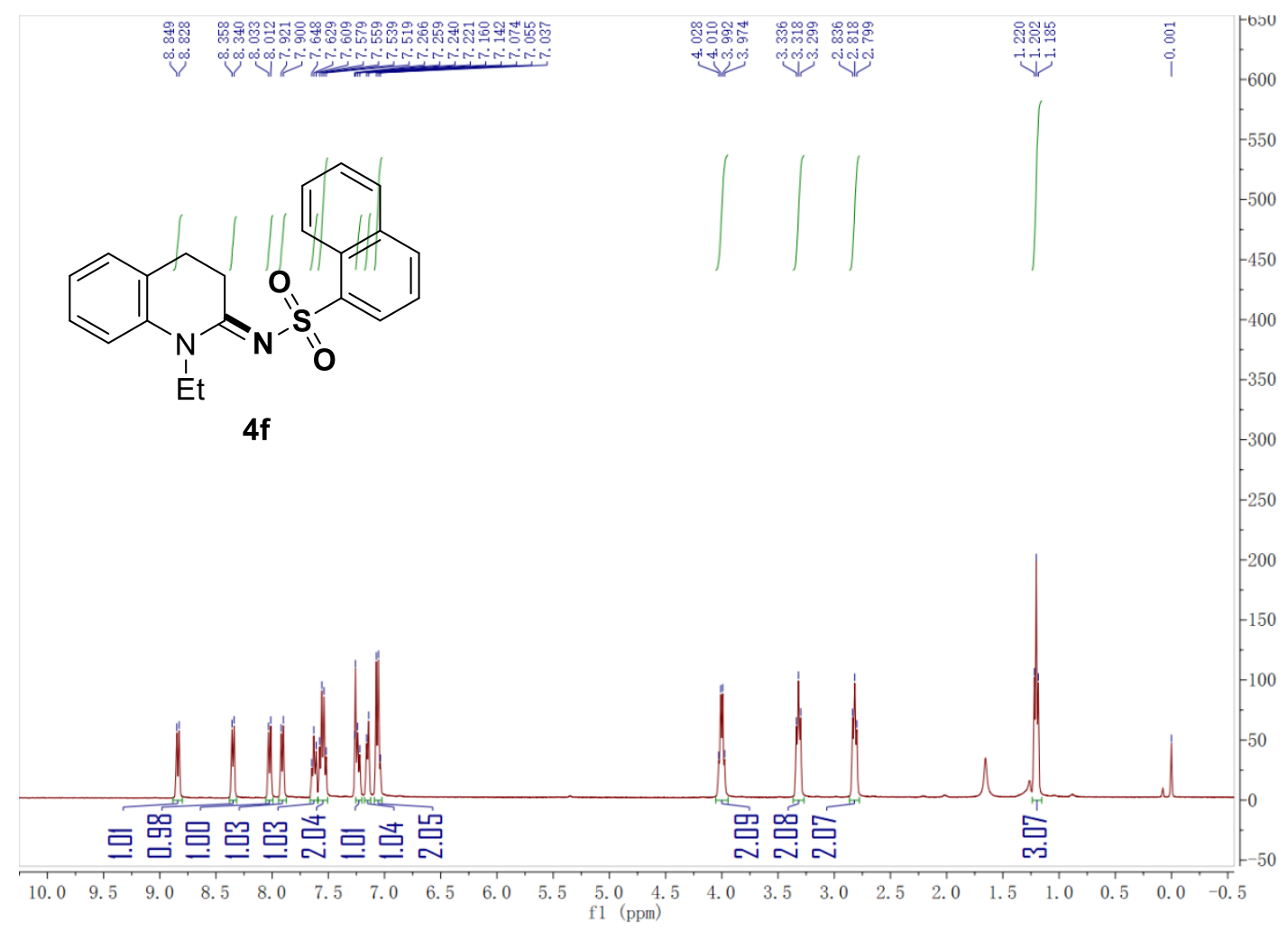

${ }^{1} \mathrm{H}$ NMR spectra $\left(400 \mathrm{MHz}, \mathrm{CDCl}_{3}\right)$ of $\mathbf{4 f}$

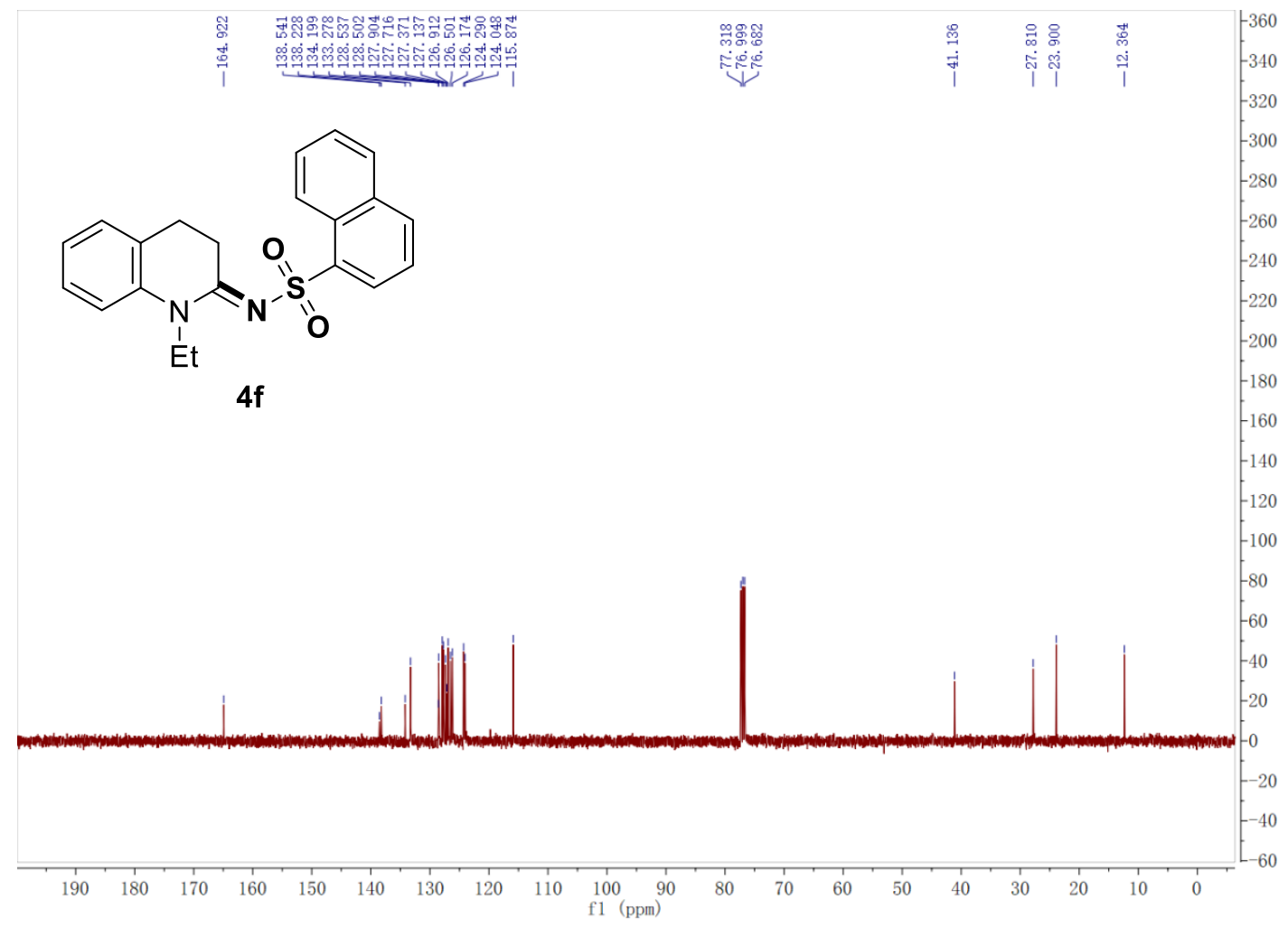

${ }^{13} \mathrm{C}$ NMR spectra $\left(101 \mathrm{MHz}, \mathrm{CDCl}_{3}\right)$ of $\mathbf{4 f}$ 


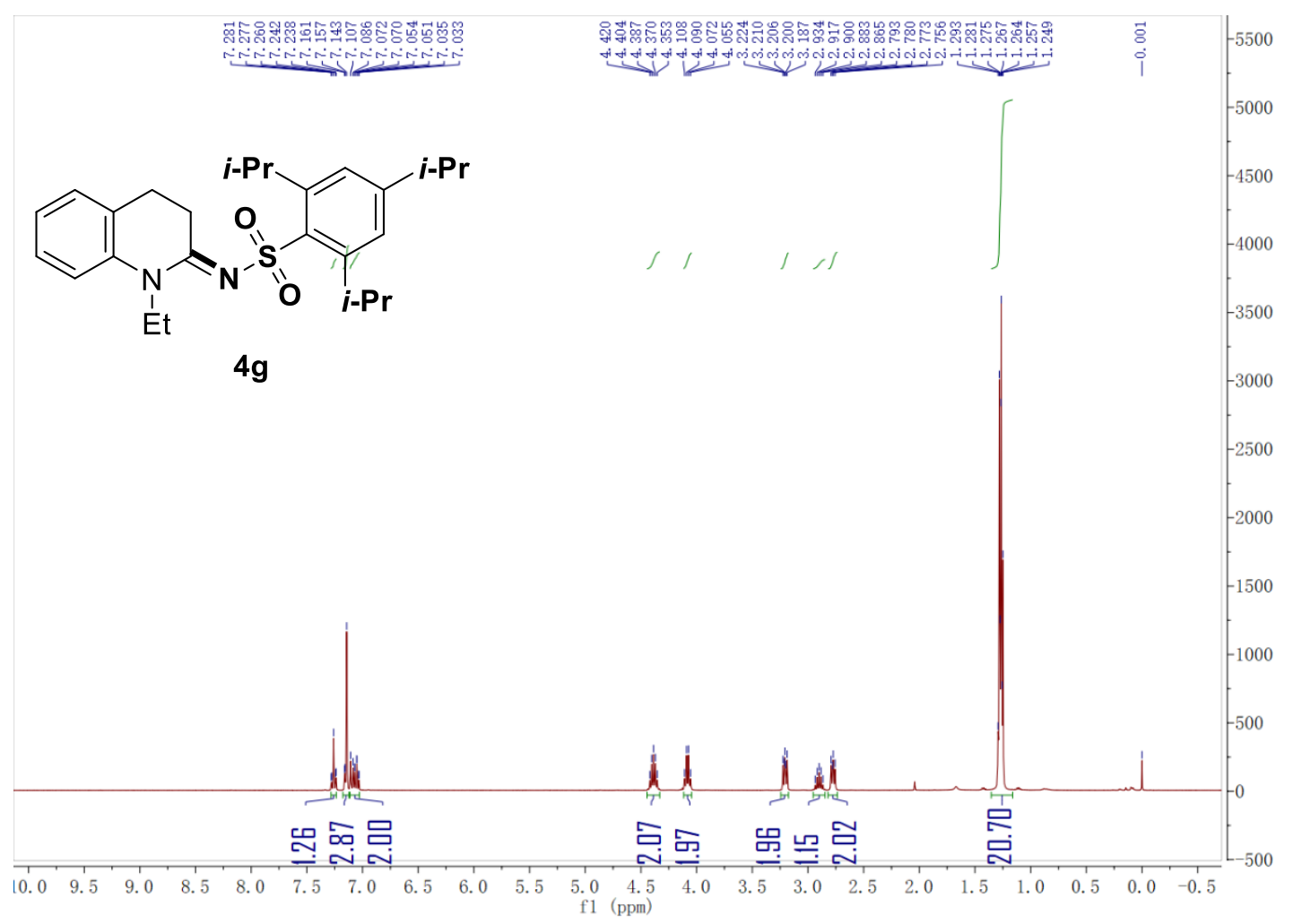

${ }^{1} \mathrm{H}$ NMR spectra (400 $\mathrm{MHz}, \mathrm{CDCl}_{3}$ ) of $\mathbf{4 g}$

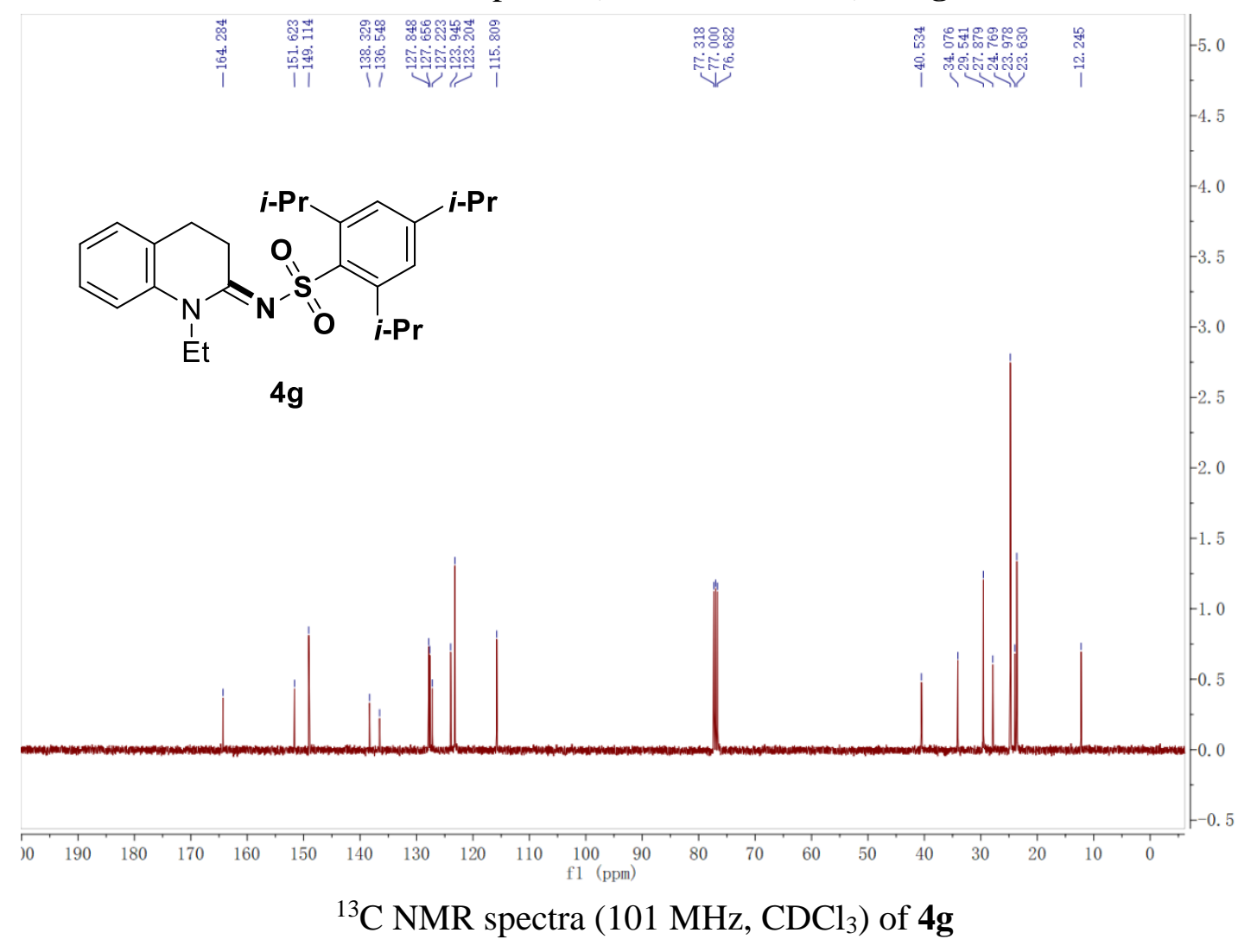




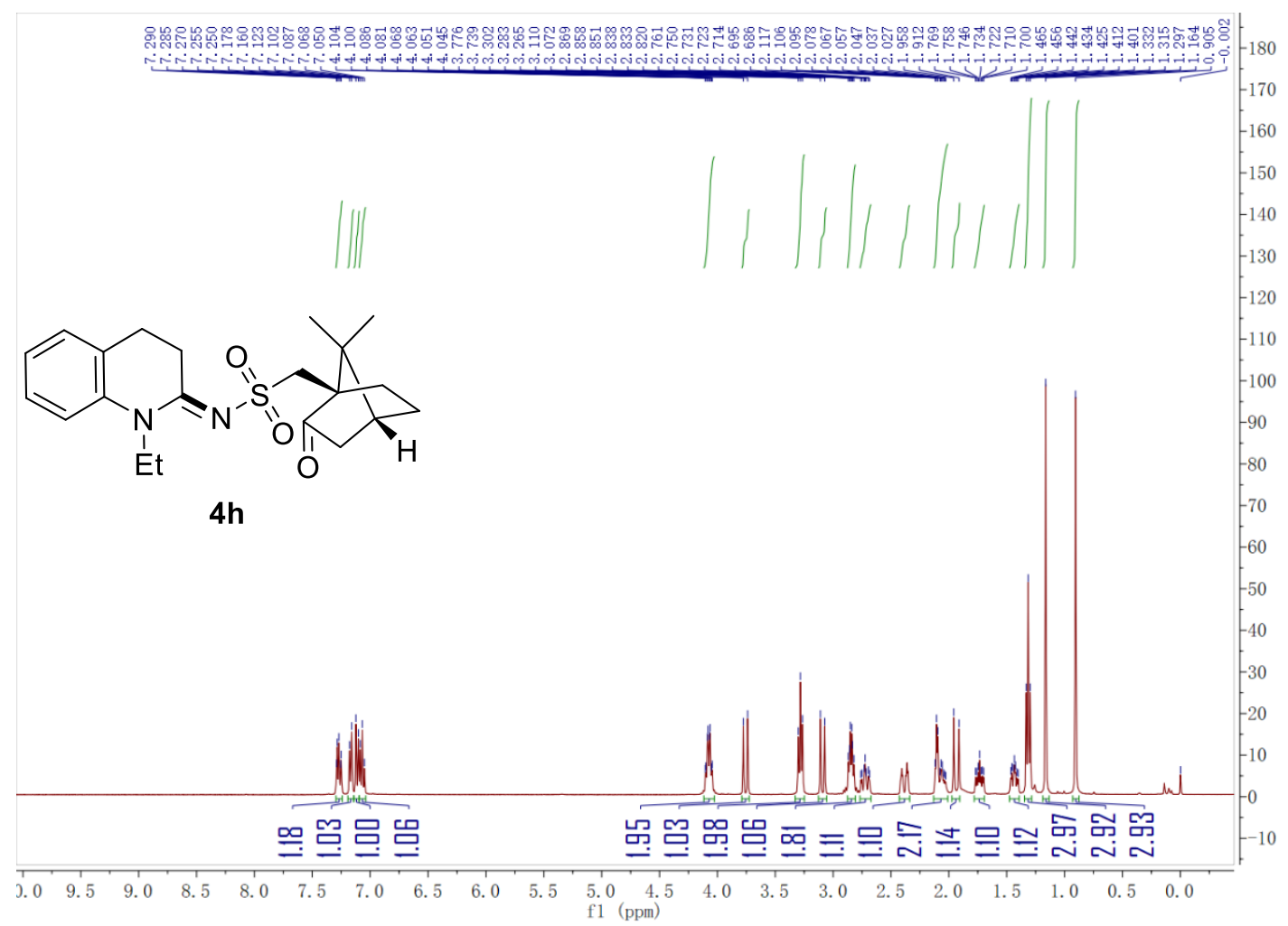

${ }^{1} \mathrm{H}$ NMR spectra $\left(400 \mathrm{MHz}, \mathrm{CDCl}_{3}\right)$ of $\mathbf{4 h}$

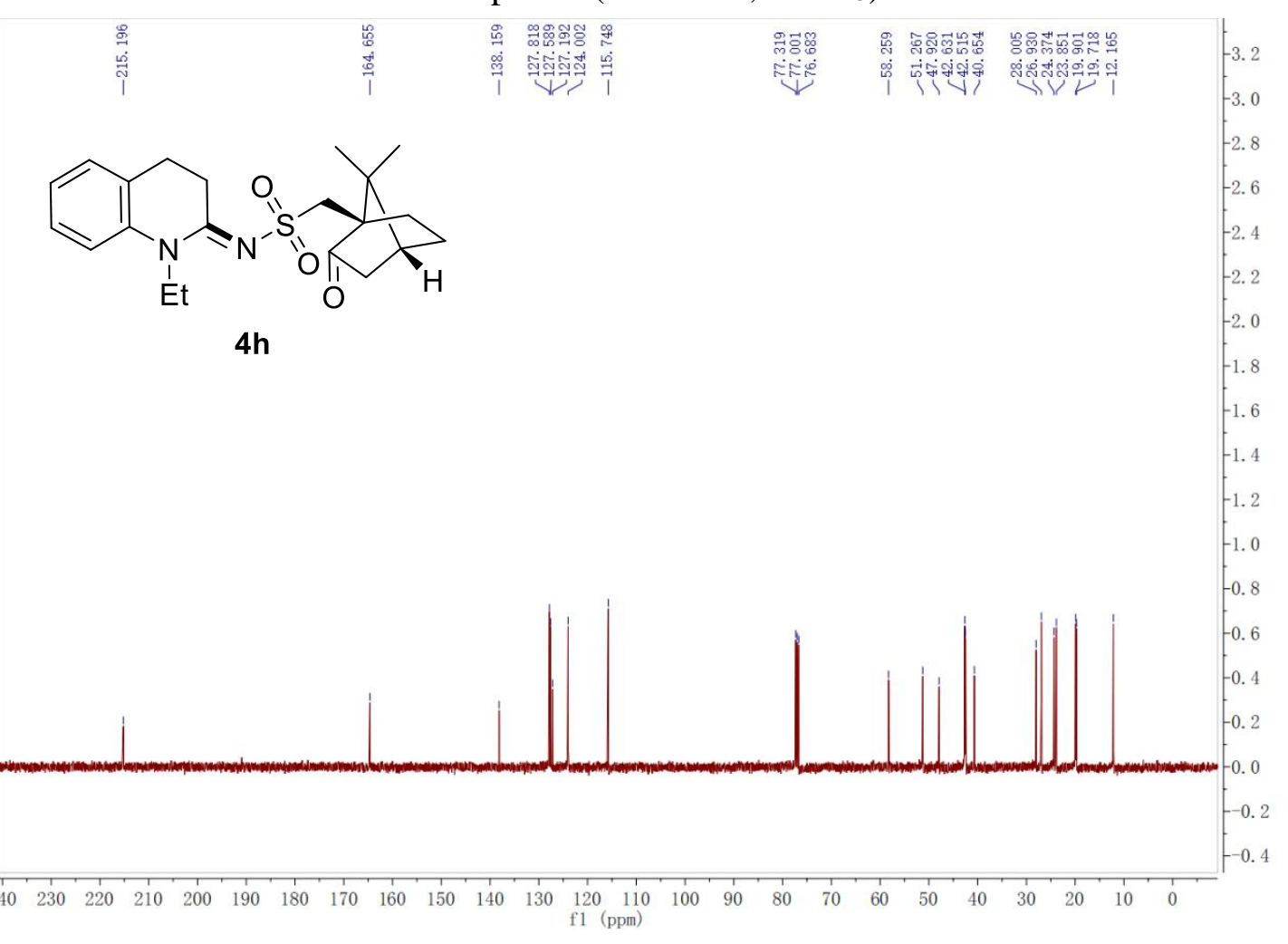

${ }^{13} \mathrm{C}$ NMR spectra $\left(101 \mathrm{MHz}, \mathrm{CDCl}_{3}\right)$ of $\mathbf{4 h}$ 


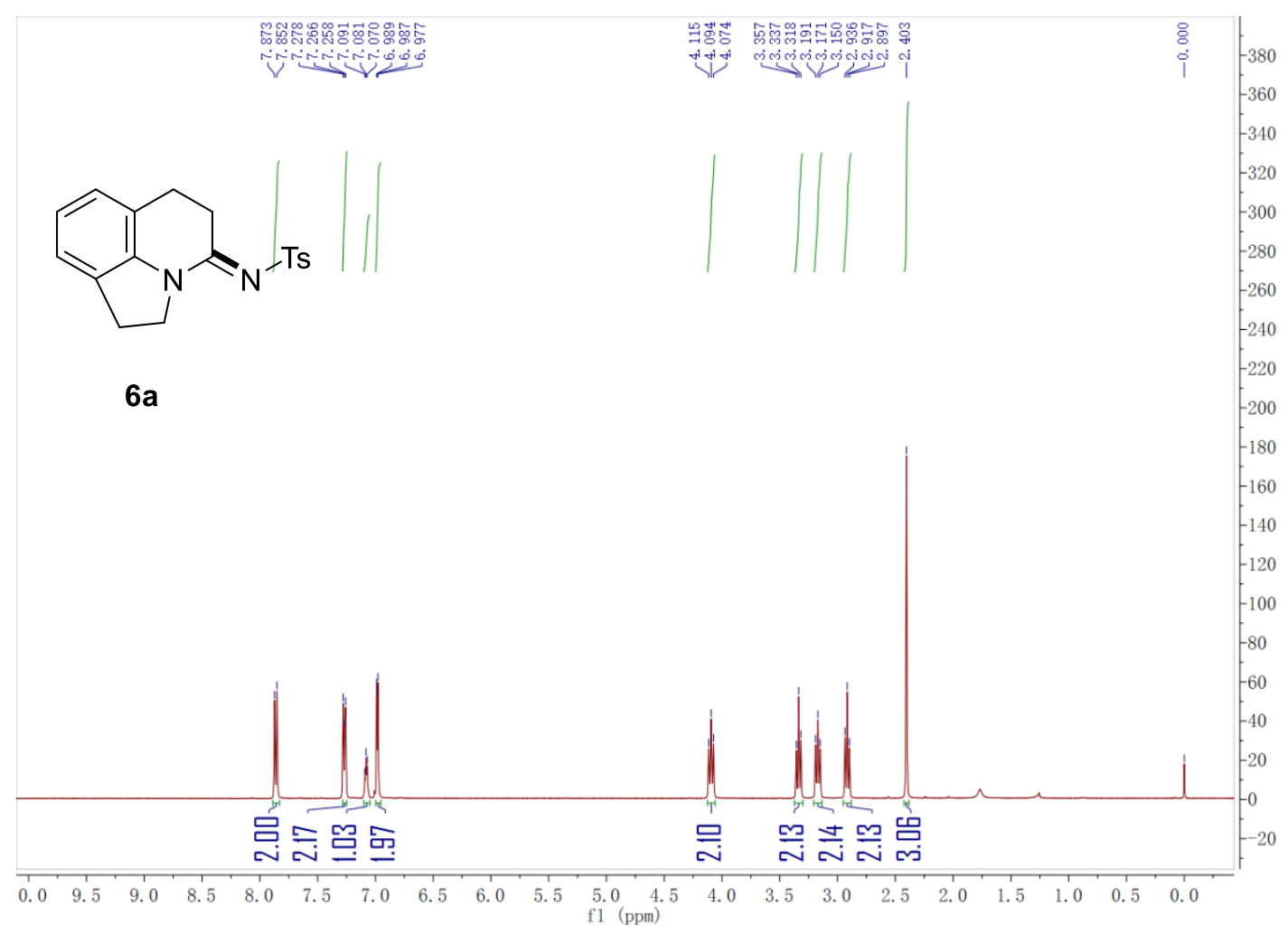

${ }^{1} \mathrm{H}$ NMR spectra (400 MHz, $\mathrm{CDCl}_{3}$ ) of $\mathbf{6 a}$

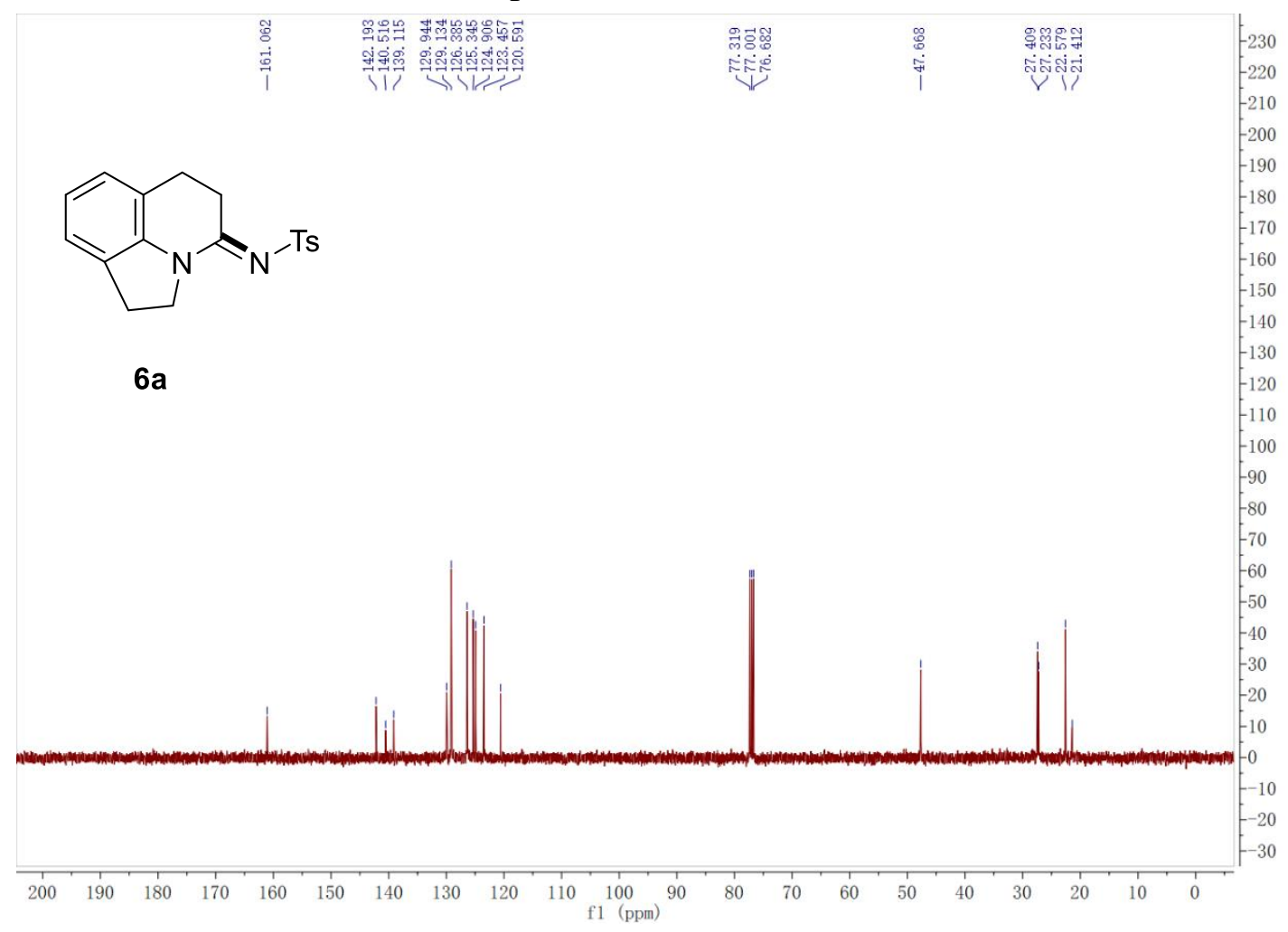

${ }^{13} \mathrm{C}$ NMR spectra $\left(101 \mathrm{MHz}, \mathrm{CDCl}_{3}\right)$ of $\mathbf{6 a}$ 


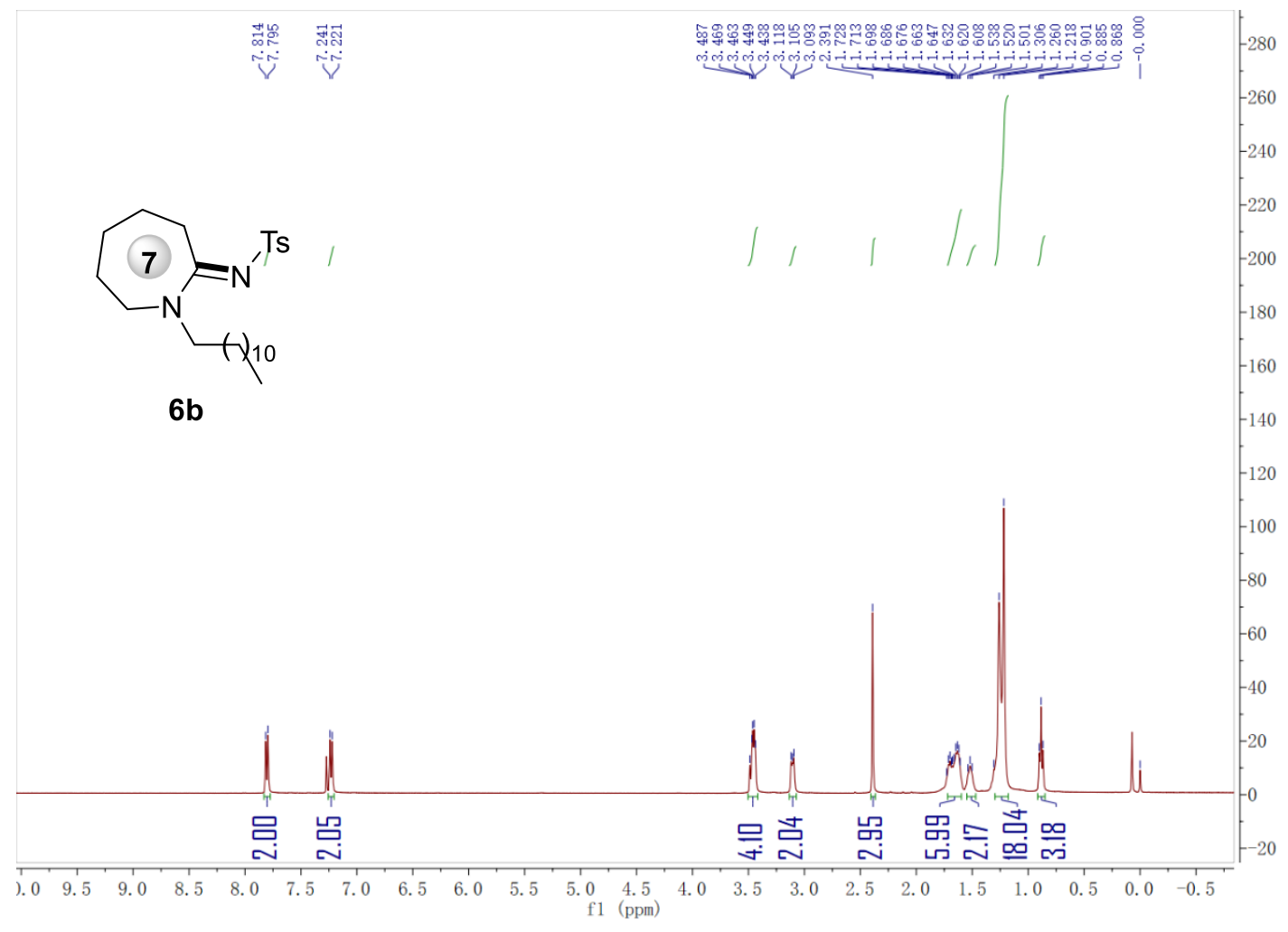

${ }^{1} \mathrm{H}$ NMR spectra $\left(400 \mathrm{MHz}, \mathrm{CDCl}_{3}\right)$ of $\mathbf{6 b}$

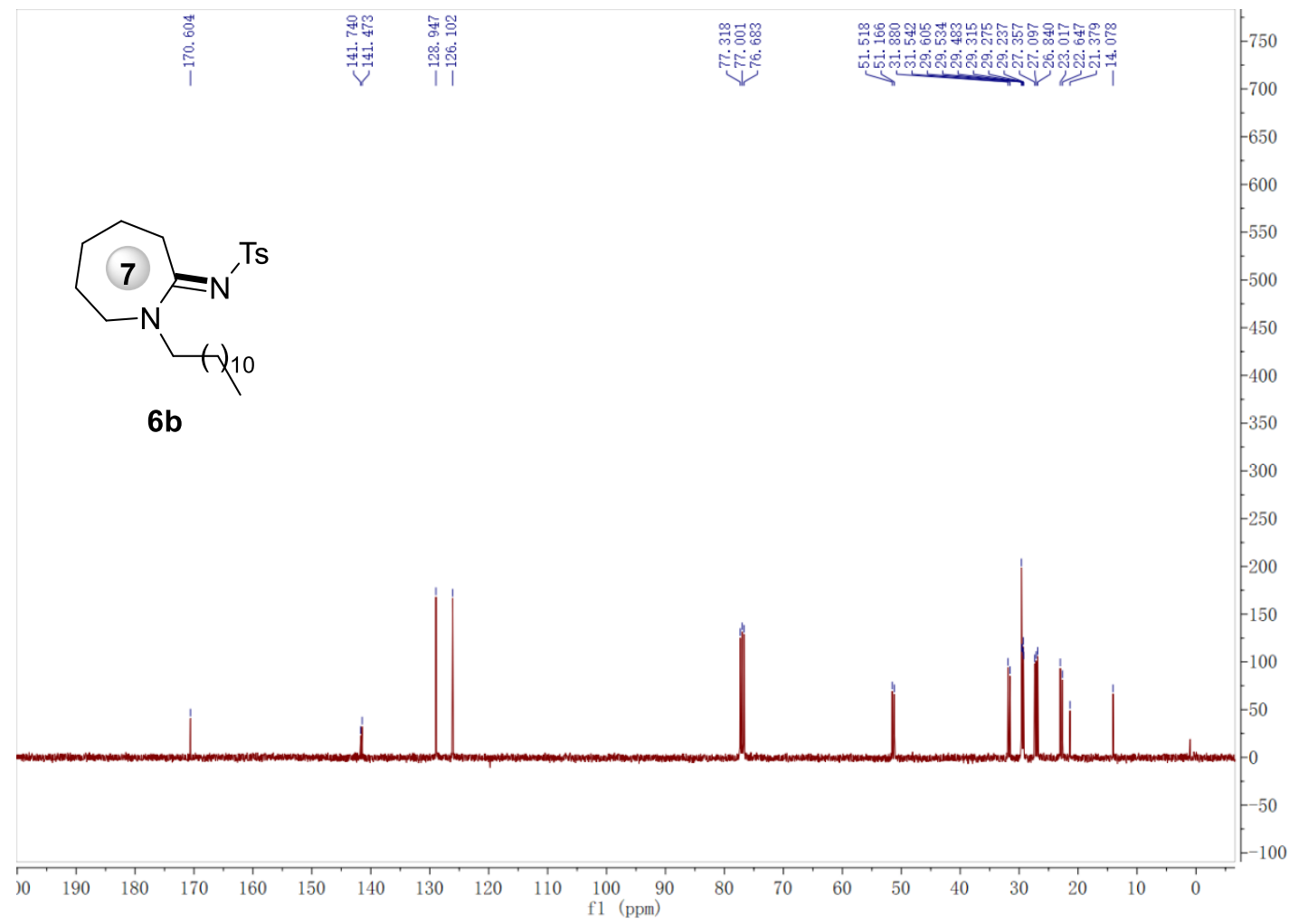

${ }^{13} \mathrm{C}$ NMR spectra $\left(101 \mathrm{MHz}, \mathrm{CDCl}_{3}\right)$ of $\mathbf{6 b}$ 

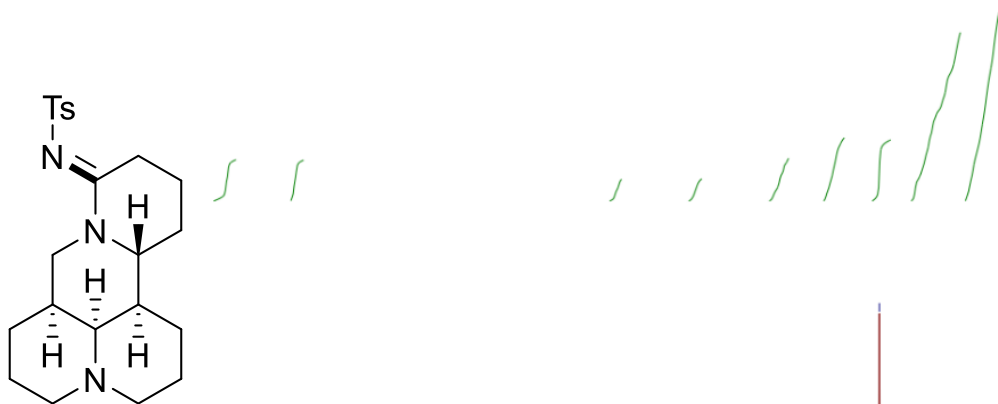

$6 c$
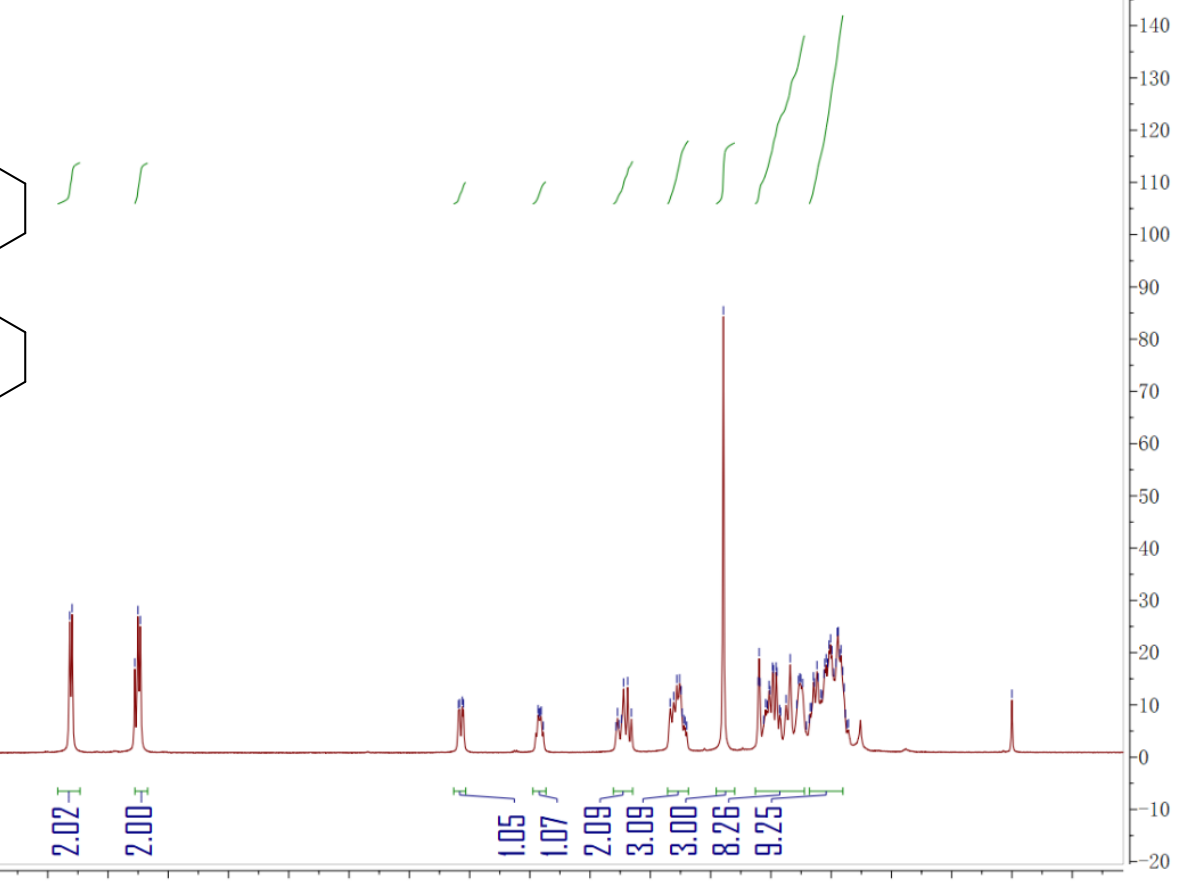

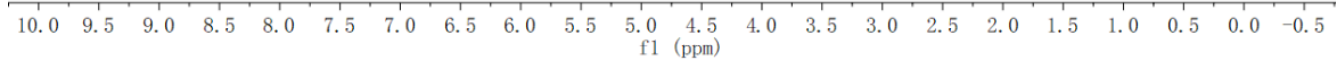

${ }^{1} \mathrm{H}$ NMR spectra (400 MHz, $\mathrm{CDCl}_{3}$ ) of $\mathbf{6 c}$

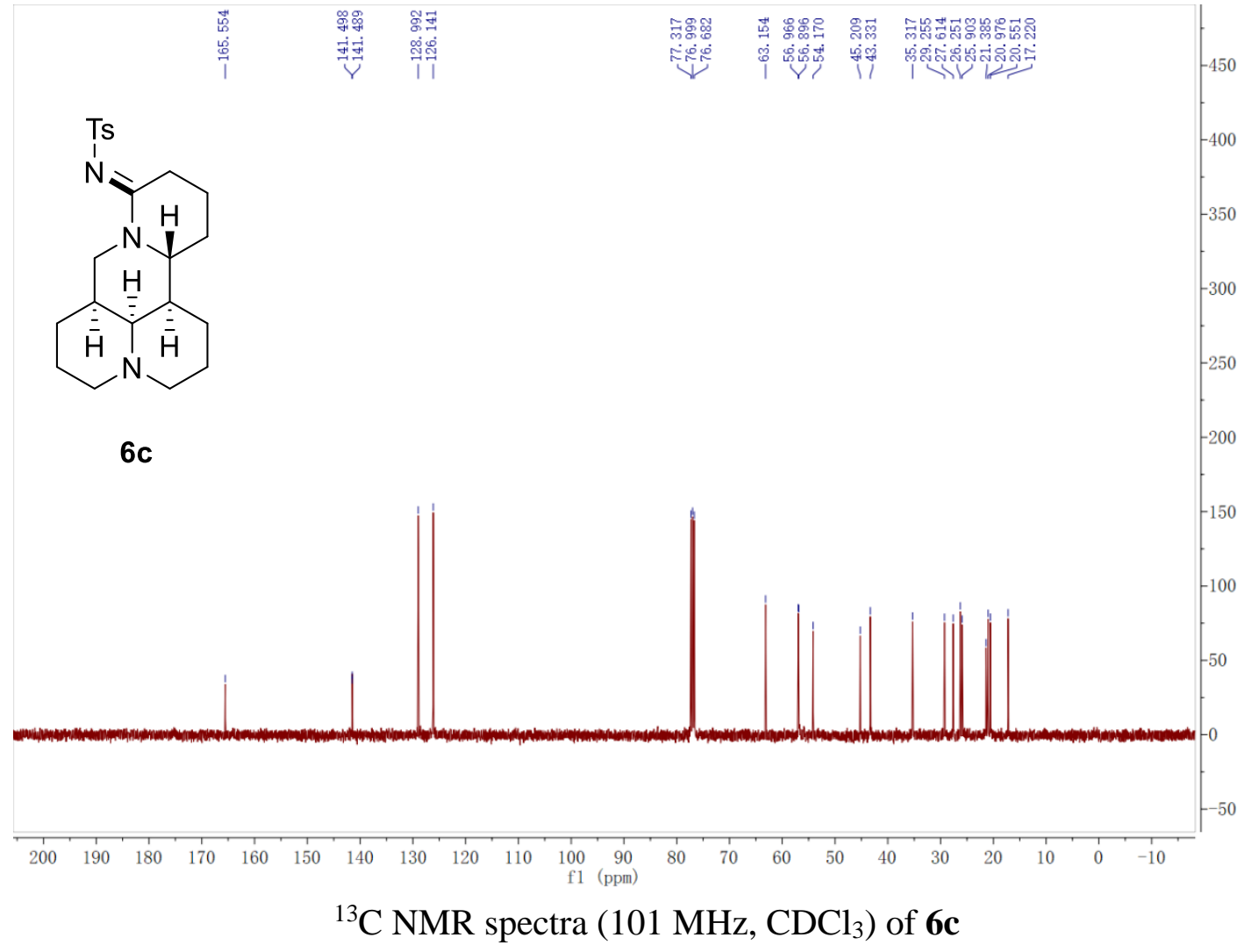




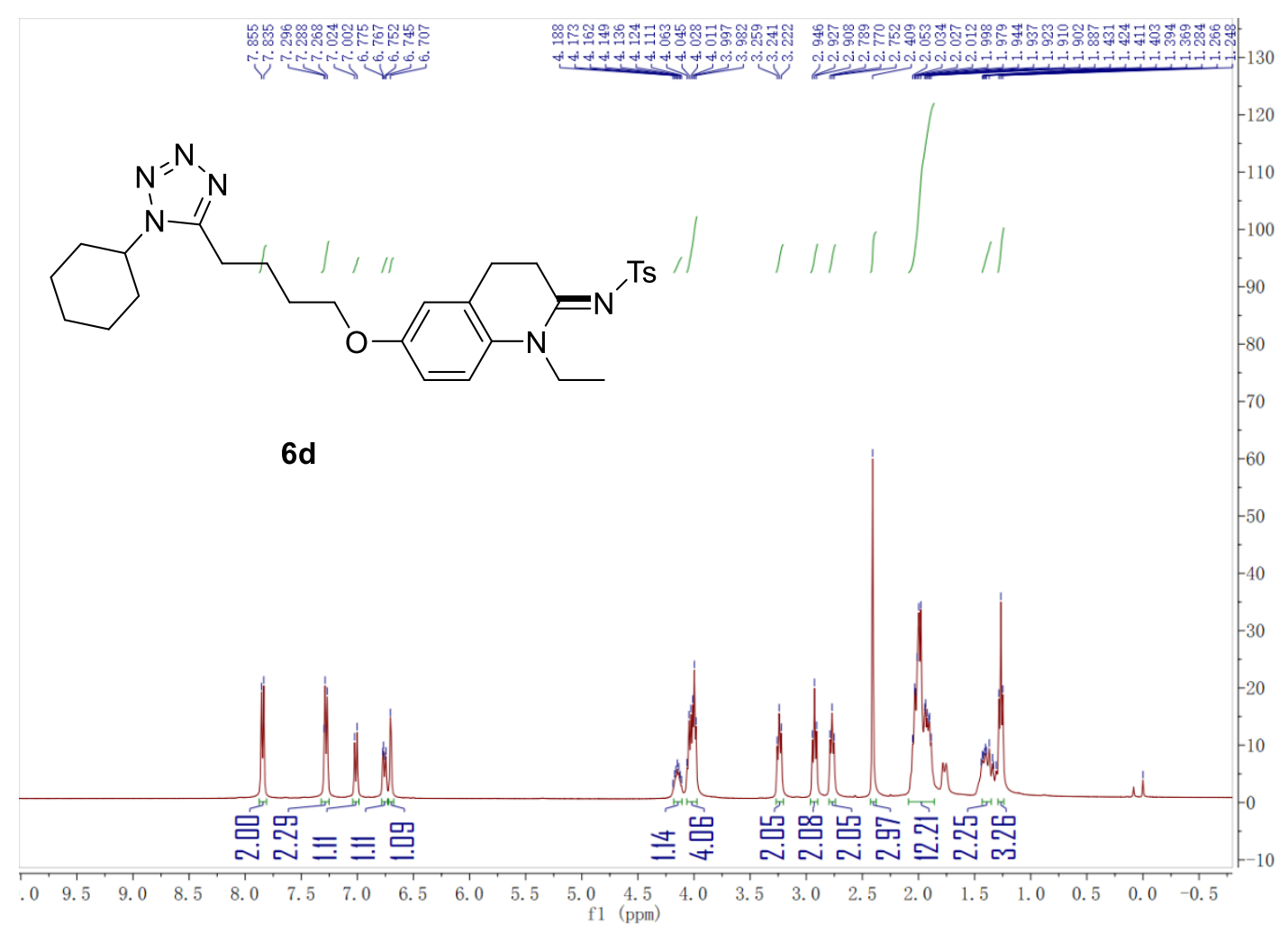

${ }^{1} \mathrm{H}$ NMR spectra $\left(400 \mathrm{MHz}, \mathrm{CDCl}_{3}\right)$ of $\mathbf{6 d}$

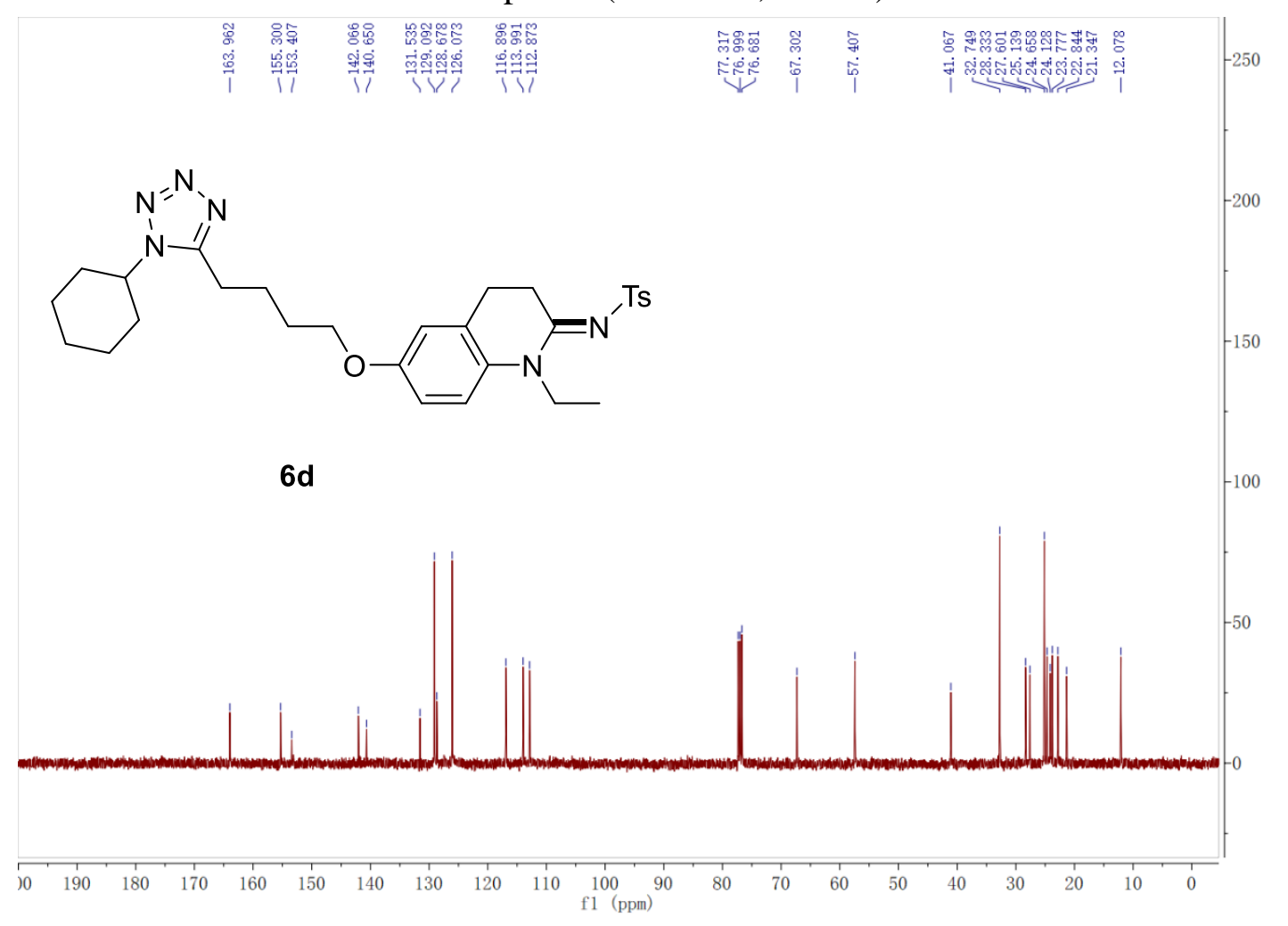

${ }^{13} \mathrm{C}$ NMR spectra $\left(101 \mathrm{MHz}, \mathrm{CDCl}_{3}\right)$ of $\mathbf{6 d}$ 


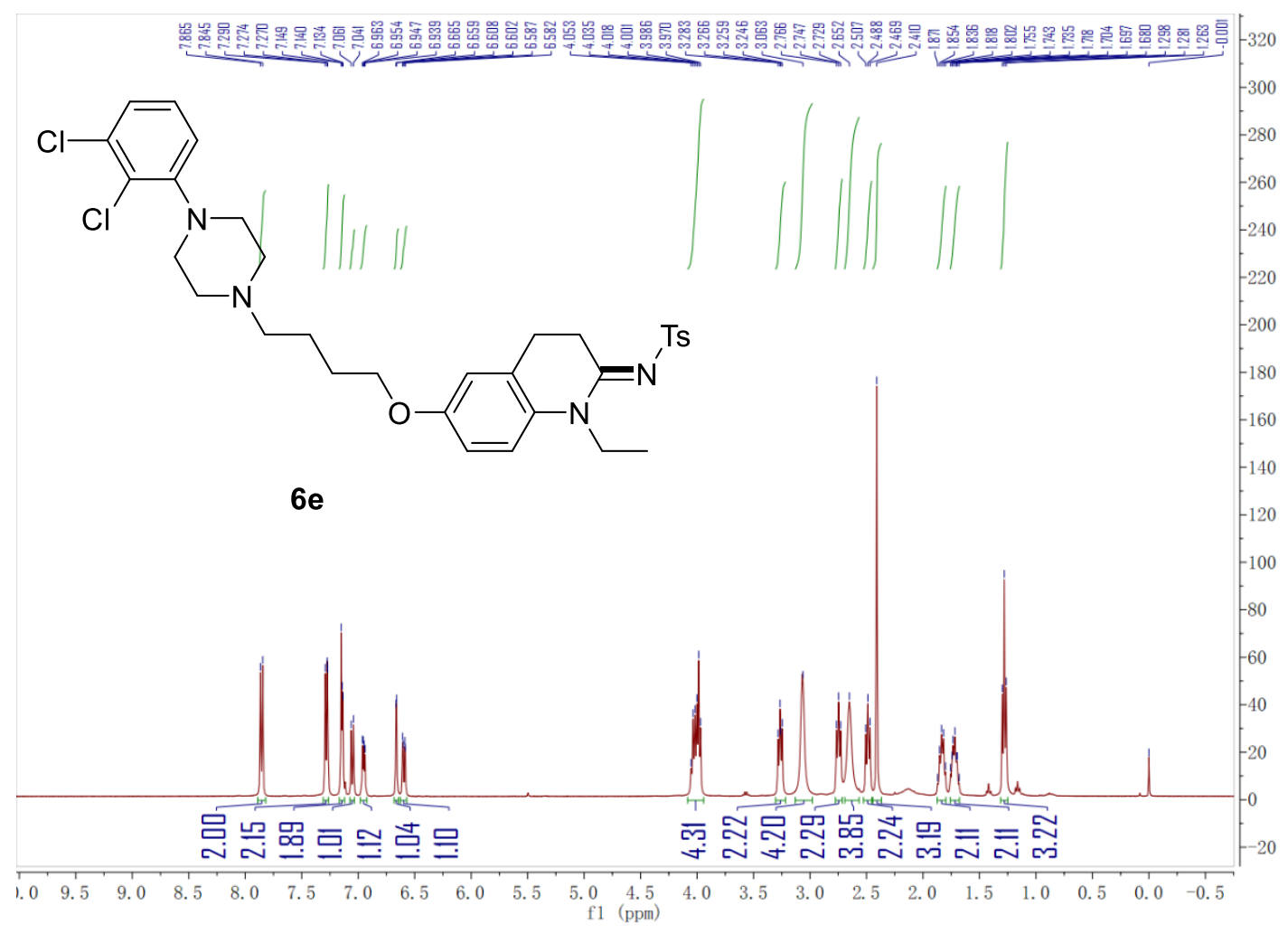

${ }^{1} \mathrm{H}$ NMR spectra $\left(400 \mathrm{MHz}, \mathrm{CDCl}_{3}\right)$ of $\mathbf{6 e}$

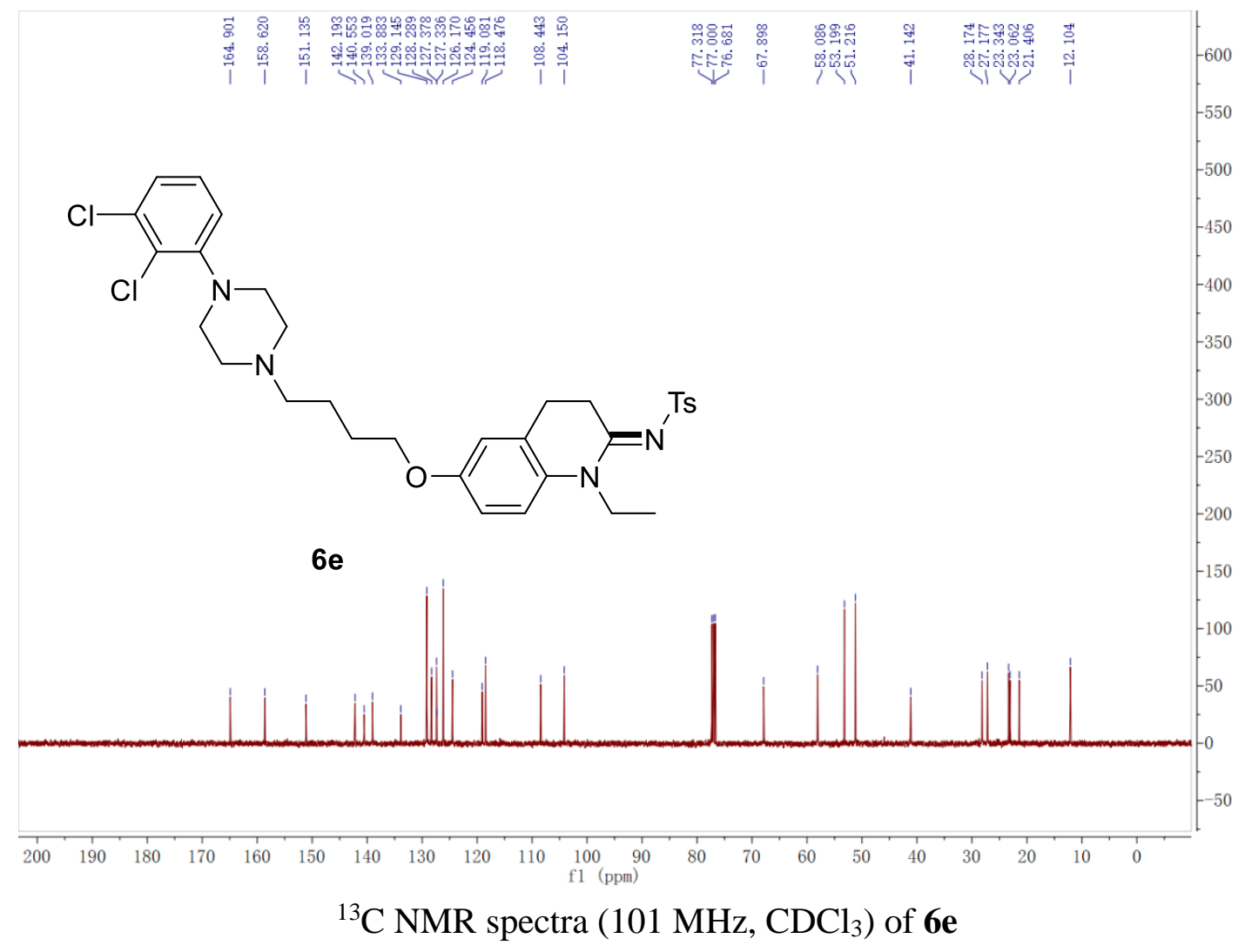

